\title{
Clinical optimization in liver surgery
}

Citation for published version (APA):

van Dam, R. M. (2014). Clinical optimization in liver surgery. [Doctoral Thesis, Maastricht University]. Maastricht University. https://doi.org/10.26481/dis.20140912rd

Document status and date:

Published: 01/01/2014

DOI:

10.26481/dis.20140912rd

Document Version:

Publisher's PDF, also known as Version of record

\section{Please check the document version of this publication:}

- A submitted manuscript is the version of the article upon submission and before peer-review. There can be important differences between the submitted version and the official published version of record.

People interested in the research are advised to contact the author for the final version of the publication, or visit the DOI to the publisher's website.

- The final author version and the galley proof are versions of the publication after peer review.

- The final published version features the final layout of the paper including the volume, issue and page numbers.

Link to publication

\footnotetext{
General rights rights.

- You may freely distribute the URL identifying the publication in the public portal. please follow below link for the End User Agreement:

www.umlib.nl/taverne-license

Take down policy

If you believe that this document breaches copyright please contact us at:

repository@maastrichtuniversity.nl

providing details and we will investigate your claim.
}

Copyright and moral rights for the publications made accessible in the public portal are retained by the authors and/or other copyright owners and it is a condition of accessing publications that users recognise and abide by the legal requirements associated with these

- Users may download and print one copy of any publication from the public portal for the purpose of private study or research.

- You may not further distribute the material or use it for any profit-making activity or commercial gain

If the publication is distributed under the terms of Article $25 \mathrm{fa}$ of the Dutch Copyright Act, indicated by the "Taverne" license above, 
CLINICAL OPTIMIZATION IN LIVER SURGERY

RONALD M. VAN DAM 
R.M. van Dam, Maastricht 2014

ISBN / EAN: $\quad 978-90-822614-0-0$

Website: http://www.clinicaloptimizationinliversurgery.nl

Design: Ronald M. van Dam

Cover: $\quad$ Nadine de Burlett, Grafische dienst Maastricht $\mathrm{UMC}^{+}$

Lay-out: $\quad$ Anke Meijs en Fabienne Meijers, Ça Va Bien Grafische Vormgeving

Production: Rob Cober, Drukkerij Maastricht $\mathrm{UMC}^{+}$

The ORANGE II trial in this thesis was financially supported by the Profileringsfonds Maastricht $\mathrm{UMC}^{+}$.

Publication of this thesis was financially supported by: B Braun Medical, Takeda Nederland, Applied Medical, Abbott, Nutricia, Roche Nederland, Krijnen Medical, Duomed, Erbe Benelux, OK Consultancy Nederland, Olympus Nederland, Covidien Nederland, Bard Benelux, Baxter, Ipsen. 

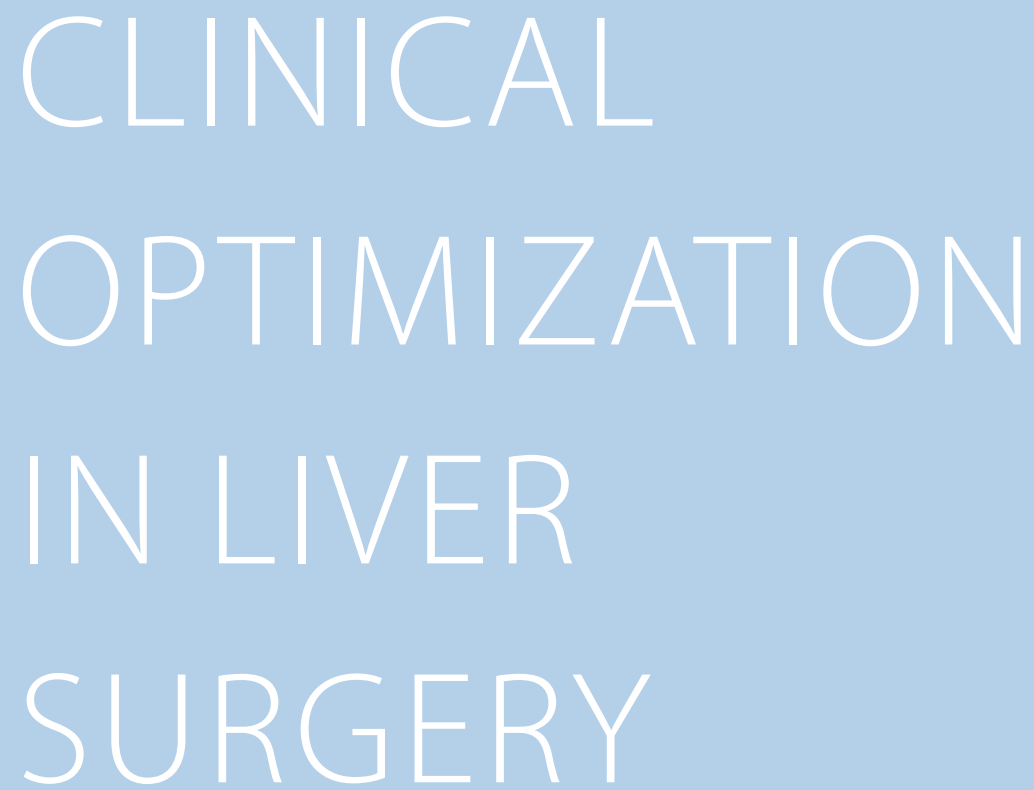

\section{PROEFSCHRIFT}

ter verkrijging van de graad van doctor aan de Universiteit Maastricht, op gezag van de Rector Magnificus, Prof dr. L.L.G Soete volgens het besluit van het College van Decanen, in het openbaar te verdedigen

op vrijdag 12 september 2014 om 14.00 uur

door 


\section{PROMOTOR}

Prof. Dr. Kees H.C. Dejong

COPROMOTOR

Dr. Steven W.M. Olde Damink

\section{BEOORDELINGS COMMISSIE}

Prof. Dr. Regina Beets-Tan (voorzitter)

Prof. Dr. Marco A.E. Marcus

Prof. Dr. Adriaan P. de Bruïne

Prof. Dr. Robert J. Porte, UMC Groningen

Prof. Dr. Ulf P. Neumann, Universitätsklinikum Aachen, Germany 
TABLE

OF CONTENTS 
CHAPTER I

General introduction

\title{
ADVANCING THE LIMITS IN LIVER SURGERY
}

CHAPTER II

Extending indication criteria

CHAPTER III

The Liver-first approach

\section{REDUCING THE RISKS OF LIVER SURGERY}

\author{
CHAPTER IV \\ ImageJ for desktop liver CT volumetry \\ CHAPTERV \\ OsiriX for virtual liver resection and CT liver volumetry
}

55

69

CHAPTER VI

Liver volume with iNtuition, ImageJ \& OsiriX 


\section{IMPROVING THE OUTCOME OF LIVER SURGERY}

Modify operations and modify perioperative care

\section{CHAPTER VI}

Enhanced recovery in liver resection

CHAPTER VIII

Enhanced recovery in laparoscopic liver surgery

CHAPTER IX

Effect of laxatives and nutritional supplements on recovery

CHAPTER X

Global adoption of enhanced recovery and laparoscopic liver surgery

CHAPTER XI

Laparoscopic vs open liver resection within an ERAS programme

\section{GENERAL DISCUSSION AND PERSPECTIVES}

\section{CHAPTER XII}

General discussion

CHAPTER XIII

Summary

CHAPTER XIV

Summary in Dutch

CHAPTER XV

List of publications

CHAPTER XVI

Acknowledgements

CHAPTER XVII

Curriculum vitae

ADDENDUM

Knowledge transfer, valorization and entrepreneurship 


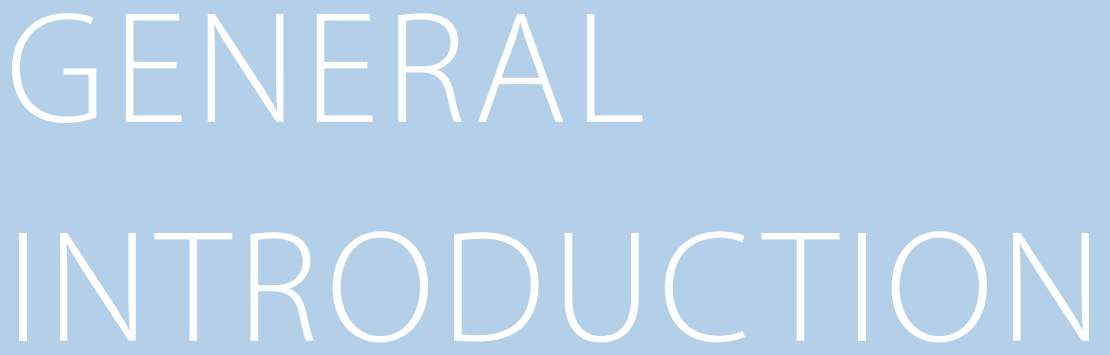

"the immediate challenge to improving the quality of surgical care is not discovering new knowledge, but rather how to integrate what we already know into practice"

Reducing variation in surgical care

D.R. Urbach, N.N. Baxter

British Medical Journal 2005, 18;330(7505):1401-2 


\section{INTRODUCTION}

\section{CHALLENGES OF MODERN LIVER SURGERY}

This thesis focuses on some of the challenges of modern liver surgery. On a day-to-day basis liver surgeons are putting in effort to improve the way patients are prepared for their surgery, how they are operated, the peri-operative care and adjuvant care they receive with the aim to improve outcome. Novel strategies are studied and suggestions are done to optimize clinical care, to make research and clinical results comparable and the design of future trials feasible.

Liver surgery can be divided in operations carried out in the context of liver transplantation and operations performed in the setting of resectional liver surgery. The present thesis focuses exclusively on resectional liver surgery. There are a whole variety of conditions that require resectional liver surgery. Most of these center around the theme of primary or secondary liver tumors. The majority of liver resections are performed for malignant tumors, and in the Western world most of these malignant tumours are metastases from colonic cancers. Also in the HPB unit of Maastricht University Medical Center, the vast majority of liver resections are performed for colorectal cancer liver metastases. It is for this reason that this thesis has an emphasis on this patient group, patients with metastatic colorectal cancer, although it is acknowledged that there is a multitude of different other tumors that may require liver resections.

\section{POPULATION AT RISK}

Approximately 9000 patients with colon cancer and 4000 patients with rectal cancer were diagnosed in the Netherlands in 2011[1]. Around 50\% of these patients will present with liver metastases during primary staging or during oncological follow up in the years after initial treatment. Up to one-fourth of patients who present with colorectal cancer have concomitant colorectal liver metastases (CRLM). Patients with these synchronous CRLM are thought to have a worse prognosis compared with patients with metachronous CRLM. Curative resection of liver metastases is historically feasible in $10-20 \%$ of metastasized colorectal cancer patients. This means that yearly between 650 and 1300 patients are potential candidates for resection of their colorectal liver metastases in the Netherlands. Moreover, around 1200 patients will be diagnosed yearly with liver-, gallbladder- or bile duct-cancer in the Netherlands. Liver resection is the only potentially curative treatment for these tumors.

\section{ORGANIZATIONAL STRUCTURE}

Traditionally liver resection was considered dangerous and it was thought to be only appropriate for patients in good clinical condition and with a very limited hepatic tumor load. Selection for resection was often judged by individual physicians at the local hospital and in the Netherlands many surgical teams were performing only a few liver resections each year. In 2005 more than 30 surgical centers in the Netherlands were performing liver surgery with 
a caseload ranging from less than 10 up to 100 per center each year. Since then, several forces led to a development of centralization of care and to the formation of multi-disciplinary teams that meet at scheduled multi-disciplinary-team meetings to discuss the optimal care of patients from a whole region. Pivotal roles in this development were a number of high impact publications that described the strong relation between case volume of surgeons and surgical teams and postoperative morbidity and mortality in oncological surgery [24]. Recently evidence has emerged also in the Netherlands that the centralisation of care for tumours that require complex surgery led to a significant reduction in peri-operative mortality and morbidity in the Netherlands [5, 6]. Until today a considerable proportion of patients with liver malignancies are still not presented at a multidisciplinary liver team and consequently not considered for liver resection. The major challenge for the HPB community is to increase the referral pattern towards highly specialised centres or to get all individual patients with liver tumors discussed in multi disciplinary regional liver meetings.

\section{EXTENDING THE INDICATIONS}

Survival after resection of various primary and secondary liver tumours has improved dramatically in the last decades. Improvements in surgical technique, optimization of peri-operative care, improvement of diagnostic imaging, preoperative liver remnant volume modulation and effectiveness of modern chemotherapy regimens have expanded the traditional limited resectability criteria. Traditionally, only patients with a maximum of 3 colorectal cancer liver metastases, not greater than $5 \mathrm{~cm}$ in diameter, located peripherally at one side of the liver, with an anticipated resection margin greater than 10 millimeter and without signs of extrahepatic disease were considered eligible for partial liver resection [7-10]. Based on these limited criteria, only $10-20 \%$ of patients with colorectal cancer liver metastases were eligible for resection. Currently, the feasibility of partial liver resection is only limited by the physical condition of a patient and the functional capacity of the remnant liver after resection. This change in surgical attitude results in larger resections and emphasises the importance of optimal pre- and peri-operative risk assessment and care.

\section{DETERMINATION OF FUTURE LIVER REMNANT VOLUME}

A small remnant liver volume has classically been perceived as an important risk factor for posthepatectomy liver failure. To reduce this risk, preoperative evaluation of the future liver remnant volume (FLRV) is thus considered critical. Volume evaluation has traditionally been based on the concept that, contrary to reality, liver function is equally distributed in all parts of the liver. However, we know from combined imaging and function studies, like hepatic scintigraphy, that in cirrhosis, biliary obstruction or focal steatosis or hepatitis, there can be a functional difference among the individual liver segments [11, 12]. In patients with a normal background liver function resections can be safely performed up to a remnant liver volume of $25 \%$ [13]. In patients with diminished liver function due to cirrhosis, cholestasis or chemotherapy-induced damage the future remnant liver volume deemed necessary for survival is 
thought to be at least 35-40 percent. Determination of FLRV is currently performed in many ways, from simple eye balling to advanced CT scan based 3D reconstruction techniques of liver volume evaluation[14]. Most of the times FLRV will be predicted by specialized hepatic radiologists using commercial radiologic image analysis software for computed tomography $(\mathrm{CT})$ volumetry or magnetic resonance imaging (MRI) volumetry. With the growing numbers of patients eligible for liver resection and to help surgical teams to assess liver remnant volumes during surgery, easy automated methods for liver volumetry in daily surgical practice are desired.

\section{DETERMINATION OF FUTURE LIVER REMNANT FUNCTION}

In recent years, awareness has increased that the volume-function relation is not always as straightforward as would be ideal for the planning of liver resections. Function depends on volume and status of remnant hepatocellular function. This makes functional assessment of the liver an increasingly popular item in liver surgery. Although accurate prediction of postresectional liver function remains difficult, there are a variety of tests used to assess this[1519]. For example, the 15-minute indocyanine green retention test (ICG R15) measures the clearance capacity of the liver and is probably the most commonly used test in patients undergoing liver resection. To determine the maximum possible extent for safe liver resection, Makuuchi et al. and Clavien et al. proposed the use of decision trees that incorporate the ICG R15 [20, 21]. More recently, the 13C-methacetin LiMAx breath test and 19Tc Mebrofenin hepatobiliary scintigraphy have been proposed for assessing hepatic functional capacity $[12,22]$. New strategies are being developed to offer treatment to patients with limited liver function and to patients with predicted critical or too small remnant liver volume. Liver resection combined with ablation of metastases and / or induction chemotherapy to reduce hepatic tumor size or tumor load, may render unresectable metastases resectable or may help to reduce the extent of liver resections. Staged resections, often combined with unilateral portal vein occlusion, are used for two-step clearance of liver metastases, to increase future remnant liver volume and to achieve a definitive R0 status. Evidently, all these strategies to assess liver volume and ensure that patients are not left with too small a liver remnant are crucially important. Above and besides this, however, there is also an important role for perioperative care to ensure the best outcomes for patients both in the short and long run.

\section{IMPROVING PERIOPERATIVE CARE}

Perioperative care in patients undergoing liver resection is still heavily influenced by dogma and tradition. Within a traditional perioperative care pathway median hospital stay following liver resection ranges from 8 to 14 days[23-26]. Morbidity is reasonably predictable, with haemorrhagic complications occurring predominantly during surgery or in the early postoperative phase, and biliary complications, intra-abdominal abscess or liver failure in the later postoperative phase. New developments in peri-operative care allow well-informed patients to participate actively in the recovery process. The Enhanced Recovery After Surgery 
$\left(\mathrm{ERAS}^{\oplus}\right)$ programme enhances postoperative recovery and consequently reduces length of hospital stay after surgery. In the last decade, the application of multimodal perioperative care protocols, commonly referred to as fast-track or enhanced recovery programmes, has achieved significant improvements in the outcome of a variety of surgical procedures. By targeting factors that delay postoperative recovery (pain, gut dysfunction and immobility), and combining a series of interventions to reduce perioperative stress and organ dysfunction, such programmes have been shown to accelerate postoperative recovery and reduce length of hospital stay. Protocols based on postoperative pain control through continuous midthoracic epidural anaesthesia, stimulation of gut motility, early physical reactivation, and limited use of catheters, tubes and drains have resulted in improved convalescence for many surgical procedures, including colonic surgery, open aortic aneurysm repair and oesophageal surgery $[27,28]$.

\section{ADVANCING SURGICALTECHNIQUES: LAPAROSCOPIC SURGERY}

In the past 2 decades, 2 parallel streams of thought have dominated the field of surgical research focusing on improving postsurgery recovery. As stipulated above, several innovators have focused on evidence-based perioperative care, as a way to enhance the patient to cope with the surgical insult. Others have highlighted the qualities of the laparoscopic, minimally invasive approach as a way to reduce the surgical insult (assuming unchanged capacity of the patient to cope with the insult). In liver surgery, unlike other fields of abdominal surgery, the laparoscopic approach has been relatively slow in finding its way into everyday practice. Laparoscopic liver surgery was first described in 1991 and it's implementation into clinical practice commenced since [29]. Recent publications from expert centers show that a substantial part of the total volume of major and minor liver resections is performed laparoscopically, and results are good[30, 31]. Laparoscopic liver resection has been claimed to result in shorter hospital length of stay (LOS), less postoperative pain, earlier recovery, and better quality of life (QOL) [32-35]. However, these results were based on patient series in selected patients and not on randomized controlled trials (RCT). Moreover, the perioperative management of the patients studied was not standardized, strategies for postoperative pain relief unclear and criteria for recovery and hospital discharge were not defined. This opens the door to a randomized assessment of the potential beneficial role of laparoscopic liver resection compared to open liver resection within a standardized perioperative care programme.

\section{TOWARDS EVIDENCE BASED LIVER SURGERY}

RCTs are scarce in liver surgery. Improvement of clinical practice and development of evidence-based liver surgery requires a continuous audit of short- and long-term outcome and large collaborative trials with well-defined endpoints, standardized perioperative care and a standardized prospective registration of intraoperative and postoperative complications. Evidence-based liver surgery should preferably be based on the results of such RCTs 
that test the effectiveness of a new treatment or technique against the standard treatment with respect to a predetermined clinically relevant endpoint. In liver surgery, single component clinical outcomes such as surgery-related mortality or specific types of morbidity are relevant primary endpoints. Ideally these endpoints should be well defined and uniformly used worldwide. RCTs using these clinical endpoints are desirable, but difficult to perform because the liver surgery specific complication rates are so low that evaluation of clinical outcomes results in very large sample sizes. The feasibility of RCTs assessing specific morbidity and mortality in liver surgery is therefore questionable and creative solutions are desired.

\section{AIMS OF THETHESIS}

The introductory overview illustrates that modern treatment of patients with liver tumours can still be improved considerably and numerous questions are currently still unanswered. The present thesis is divided into three sections, focusing on three major topics. In the first section, novel strategies to push the limits of liver resection are studied. In the second section, strategies are explored to reduce the risks of liver resection by predicting the volume of the future remnant liver using open source software for CT liver volumetry and virtual hepatectomy. In the third section, ways to improve the outcome of liver resection through modification of operation techniques and by modification of perioperative care are evaluated. In this context, the AIMS of the present thesis condense down to these three sections.

\section{SECTION 1 - ADVANCING THE LIMITS IN LIVER SURGERY}

The aims of this section included:

To determine the clinical efficacy of extended criteria for liver surgery versus classical limited criteria. Special emphasis wa placed on overall survival, post-operative morbidity and mortality, disease free survival and percentage Ro resections.

To determine the feasibility and outcome of reversing the surgery sequence in patients with synchronous colorectal liver metastases. The effects of the so-called liver first approach were studied, in which the colorectal cancer liver metastases were resected before resection of the primary rectal cancer.

\section{SECTION 2 - REDUCING THE RISKS OF LIVER SURGERY USING HEPATIC CT VOLUMETRY TO PREDICTTHE FUTURE REMNANT LIVER}

The aims of this section of the thesis were:

To study the accuracy of determining liver volumes using the free-downloadable software package ImageJ for hepatic CT volumetry. Volumes of virtual liver resection specimens measured with ImageJ were compared with specimen weights and calculated volumes obtained during pathology examination after resection. 
To study the accuracy of a user-friendly, freely downloadable open source image analysis software package, OsiriX for predicting liver resection volume in patients undergoing partial hepatectomy.

To study the accuracy and time consumption of measuring liver tumour volumes and future resection volumes using free-downloadable software (ImageJ and OsiriX) versus professional image analysis software (Aquarius iNtuition software).

\section{SECTION 3 - IMPROVING THE OUTCOME OF LIVER SURGERY: MODIFY OPERATION TECHNIQUES AND MODIFY PERI-OPERATIVE CARE}

The aims of this section were:

To determine the feasibility of implementing the ERAS ${ }^{\circledR}$ enhanced recovery programme in patients undergoing liver surgery.

To determine the effect of a multimodal fast-track programme on outcomes in laparoscopic liver surgery.

To determine the additional effects of laxatives and oral nutritional supplements within the ERAS $^{\circledast}$ enhanced recovery after surgery protocol following liver resection on functional reovery and gastrointestinal function.

To survey the experience with and (evidence-based) implementation of laparoscopic liver surgery and enhanced recovery or "fast-track" recovery programmes within the global HPB community.

To assess the effect of the laparoscopic technique on outcomes in patients undergoing hepatic left lateral sectionectomy or hemihepatectomy within an enhanced recovery ERAS ${ }^{\circledast}$ programme.

These three sections and the individual chapters contained therein are followed by a general discussion. This general discussion is based on the results of this thesis, as well as on several other studies that the author of this thesis was involved in as an investigator or supervisor in the past 10 years. The amalgamate of results is discussed against the background of available literature with a focus to provide ways to work towards Evidence Based Liver Surgery. This entails amongst others a detailed overview of the feasibility to perform RCTs in liver surgery, ways to achieve uniformity in definitions of liver surgery specific complications, and the use of composite endpoints in liver surgery to facilitate RCTs in liver surgery. Finally, an RCT is proposed taking all of these into account, which is designed to assess the potential beneficial role of laparoscopic liver resection compared to open liver resection within a standardized perioperative care program. 


\section{REFERENCES}

1. IKNL. Dutch Colorectal \& Hepatobiliairy Cancer Incidence 2011. 2011 [cited 2013; Available from: http:// www.cijfersoverkanker.nl.

2. Gouma, D.J., et al., Rates of complications and death after pancreaticoduodenectomy: risk factors and the impact of hospital volume. Ann Surg, 2000. 232(6): p. 786-95.

3. van Lanschot, J.J., et al., Hospital volume and hospital mortality for esophagectomy. Cancer, 2001. 91(8): p. 1574-8.

4. Ho, V. and M.J. Heslin, Effect of hospital volume and experience on in-hospital mortality for pancreaticoduodenectomy. Ann Surg, 2003. 237(4): p. 509-14.

5. van Heek, N.T., et al., Hospital volume and mortality after pancreatic resection: a systematic review and an evaluation of intervention in the Netherlands. Ann Surg, 2005. 242(6): p. 781-8, discussion 788-90.

6. de Wilde, R.F., et al., Impact of nationwide centralization of pancreaticoduodenectomy on hospital mortality. Br J Surg, 2012. 99(3): p. 404-10.

7. Nordlinger, B., et al., Surgical resection of colorectal carcinoma metastases to the liver. A prognostic scoring system to improve case selection, based on 1568 patients. Association Francaise de Chirurgie. Cancer, 1996. 77(7): p. 1254-62.

8. Fong, Y., et al., Clinical score for predicting recurrence after hepatic resection for metastatic colorectal cancer: analysis of 1001 consecutive cases. Ann Surg, 1999. 230(3): p. 309-18; discussion 318-21.

9. Altendorf-Hofmann, A. and J. Scheele, A critical review of the major indicators of prognosis after resection of hepatic metastases from colorectal carcinoma. Surg Oncol Clin N Am, 2003. 12(1): p. 165-92, xi.

10. Sharma, S., C. Camci, and N. Jabbour, Management of hepatic metastasis from colorectal cancers: an update. J Hepatobiliary Pancreat Surg, 2008. 15(6): p. 570-80.

11. Erdogan, D., et al., Preoperative assessment of liver function: a comparison of 99mTc-Mebrofenin scintig- raphy with indocyanine green clearance test. Liver Int, 2004. 24(2): p. 117-23.

12. de Graaf, W., et al., Nuclear imaging techniques for the assessment of hepatic function in liver surgery and transplantation. J Nucl Med, 2010. 51(5): p. 742-52.

13. Schindl, M.J., et al., The adaptive response of the reticuloendothelial system to major liver resection in humans. Ann Surg, 2006. 243(4): p. 507-14.

14. Radtke, A., et al., Computer-assisted surgery planning for complex liver resections: when is it helpful? A single-center experience over an 8-year period. Ann Surg, 2010. 252(5): p. 876-83.

15. Armuzzi, A., et al., Review article: breath testing for human liver function assessment. Aliment Pharmacol Ther, 2002. 16(12): p. 1977-96.

16. Fazakas, J., et al., Evaluation of liver function for hepatic resection. Transplant Proc, 2006. 38(3): p. 798-800.

17. Gill, R.A., et al., Aminopyrine breath test predicts surgical risk for patients with liver disease. Ann Surg, 1983. 198(6): p. 701-4.

18. Schneider, P.D., Preoperative assessment of liver function. Surg Clin North Am, 2004. 84(2): p. 355-73.

19. Seyama, Y. and N. Kokudo, Assessment of liver function for safe hepatic resection. Hepatol Res, 2009. 39(2): p. 107-16.

20. Clavien, P.A., et al., Strategies for safer liver surgery and partial liver transplantation. N Engl J Med, 2007. 356(15): p. 1545-59.

21. Makuuchi, M., et al., Surgery for small liver cancers. Semin Surg Oncol, 1993. 9(4): p. 298-304.

22. Stockmann, M., et al., Prediction of postoperative outcome after hepatectomy with a new bedside test for maximal liver function capacity. Ann Surg, 2009. 250(1): p. 119-25.

23. Capussotti, L., et al., Randomized clinical trial of liver resection with and without hepatic pedicle clamping. $\mathrm{Br}$ J Surg, 2006. 93(6): p. 685-9.

24. Dimick, J.B., et al., National trends in the use and outcomes of hepatic resection. J Am Coll Surg, 2004. 199(1): p. 31-8. 
25. Jarnagin, W.R., et al., Improvement in perioperative outcome after hepatic resection: analysis of 1,803 consecutive cases over the past decade. Ann Surg, 2002. 236(4): p. 397-406; discussion 406-7.

26. Pessaux, P., et al., Randomized clinical trial evaluating the need for routine nasogastric decompression after elective hepatic resection. Br J Surg, 2007. 94(3): p. 297303.

27. Fearon, K.C., et al., Enhanced recovery after surgery: a consensus review of clinical care for patients undergoing colonic resection. Clin Nutr, 2005. 24(3): p. 466-77.

28. Kehlet, H. and D.W. Wilmore, Multimodal strategies to improve surgical outcome. Am J Surg, 2002. 183(6): p. 630-41.

29. Reich, H.M., F.; DeCaprio, J. and Budin, R., Laparoscopic excision of benign liver lesions. Obstet Gynecol, 1991. 78(5.2): p. 956-8.

30. Nguyen, K.T., T.C. Gamblin, and D.A. Geller, World review of laparoscopic liver resection-2,804 patients. Ann Surg, 2009. 250(5): p. 831-41.

31. Bryant, R., et al., Laparoscopic liver resection-understanding its role in current practice: the Henri Mondor Hospital experience. Ann Surg, 2009. 250(1): p. 103-11.

32. Abu Hilal, M., et al., Laparoscopic versus open left lateral hepatic sectionectomy: A comparative study. Eur J Surg Oncol, 2008. 34(12): p. 1285-8.

33. Lee, K.F., et al., Laparoscopic versus open hepatectomy for liver tumours: a case control study. Hong Kong Med J, 2007. 13(6): p. 442-8.

34. Laurence, J.M., et al., Laparoscopic hepatectomy, a systematic review. ANZ J Surg, 2007. 77(11): p. 948-53.

35. Alkari, B., A. Owera, and B.J. Ammori, Laparoscopic liver resection: preliminary results from a UK centre. Surg Endosc, 2008. 22(10): p. 2201-7. 


\section{ADVANCING THE LIMITS IN LIVER SURGERY}

OUTCOMES OF EXTENDED VERSUS LIMITED INDICATIONS FOR PATIENTS UNDERGOING LIVER RESECTION FOR COLORECTAL CANCER LIVER METASTASES

R.M. van Dam, T.M. Lodewick, M.A.J van den Broek, M.C. de Jong, J.W. Greve, R.L.H. Jansen, M.H.A. Bemelmans, U.P. Neumann, S.W.M. Olde Damink, C.H.C Dejong.

HPB 2014, 16(6):550-9

THE LIVER-FIRST APPROACH FOR SYNCHRONOUS COLORECTAL LIVER METASTASIS: A 5-YEAR SINGLE-CENTRE EXPERIENCE

M.C. de Jong, R.M. van Dam, M. Maas, M.H.A. Bemelmans, S.W.M. Olde Damink, G.L. Beets, C.H.C. Dejong

HPB 2011, 13(10): 745-752 

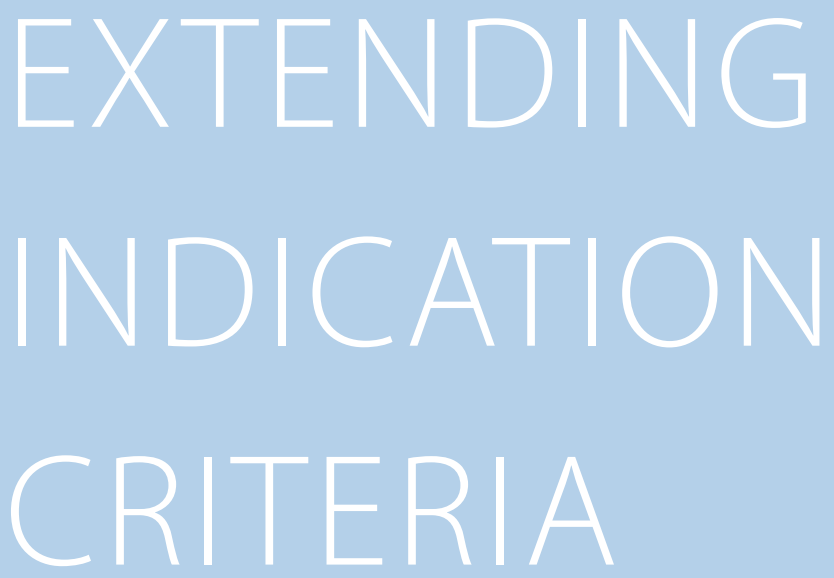

\section{PUBLISHED AS}

Outcomes of extended versus limited indications for patients undergoing liver resection for colorectal cancer liver metastases.

R.M. van Dam T.M. Lodewick, M.A.J van den Broek, M.C. de Jong, J.W. Greve, R.L.H. Jansen, M.H.A. Bemelmans, U.P. Neumann, S.W.M. Olde Damink, C.H.C Dejong.

HPB 2014, 16(6):550-9

DOI: dx.doi.org/10.1111/hpb.12181 


\section{ABSTRACT}

\section{BACKGROUND}

Currently, resection criteria for colorectal cancer liver metastases (CRCLM) are only limited by remnant liver function. We compared morbidity and survival after partial hepatectomy with limited or extended indication criteria.

\section{METHODS}

Patients undergoing liver resection for CRCLM with limited $(n=169)$ or extended indication criteria $(n=129)$ between 1991 and 2010 were retrospectively identified in a prospectively collected single center database. Limited indication criteria were defined as less than three unilateral, not centrally located liver metastases in the absence of extra hepatic metastases. The extended criteria were only limited by predicted remnant liver volume and patients fitness. Data on co-morbidity, resection margin, short- and long-term morbidity, disease free (DFS) and overall survival were compared.

\section{RESULTS}

Patients with limited indications had less major complications ( $19.5 \%$ vs $33.1 \%, p<0.01$ ), longer overall survival of 68.8 months ( $\mathrm{Cl} 46.5-91.1)$ vs 41.4 months (Cl 33.4-49.0, $\mathrm{p}=<0.001$ ), and longer median DFS of 22.0 months (Cl 15.8-28.2) vs 10.2 months (Cl 8.4-11.9, $\mathrm{p}<0.001$ ) compared to the extended indication group. Cure rates, defined as 10-year DFS, were $35.5 \%$ and $15.8 \%$ respectively. Fewer patients in the extended indication group underwent an R0 resection $(92.9 \%$ vs $77.5 \%, \mathrm{p}<0.001)$. Only $17 \%$ of all $\mathrm{R} 1$ resected patients had recurrences at the transection plane.

\section{CONCLUSION}

Partial hepatectomy for CRCLM with extended indications seems justified but is associated with higher complication rates, earlier recurrence and lower overall survival compared to limited indications. However, median 5-year survival was substantial and cure was achieved in $15.8 \%$ of patients. 


\section{INTRODUCTION}

During the last decade, the limited criteria for partial liver resection have been replaced by more extended indication criteria. Improvements in surgical technique, optimization of peri-operative care, improvement of diagnostic imaging, preoperative liver remnant volume modulation and effectiveness of modern chemotherapy regimens have boosted the widening of resectability criteria. Traditionally, only patients with a maximum of 3 colorectal cancer liver metastases, located peripherally at one side of the liver, with an anticipated resection margin greater than 10 millimeter and without signs of extrahepatic disease were considered eligible for partial liver resection [1-4]. Based on these limited criteria, only 10$20 \%$ of patients with colorectal cancer liver metastases were eligible for resection. Recent studies have shown that liver resection in patients with multiple and / or bilateral colorectal liver metastases results in overall 5-years survival rates between 23 and 51\% [4, 5]. Moreover, centrally located liver metastases are no longer a contraindication for liver surgery. In patients with a normal functioning liver, extended hemihepatectomies can be performed safely and mesohepatectomy or central liver resection is an alternative for extended hemihepatectomy when parenchymal loss needs to be minimized [6]. If resectable extrahepatic metastases are present, resection can be offered with 5-year overall survival rates up to $28 \%[6,7]$. Thus, liver resection criteria for colorectal cancer liver metastases (CRCLM) are at present only limited by an anticipated R0 status and an adequate functional liver remnant. Patients in good general health, with technically resectable metastatic disease limited to the liver, regional lymph nodes or / and lungs, are considered for resection regardless of associated clinical predictive factors [8-10]. Parallel to the expanding indications for liver resection also new strategies to improve resectability have been popularized [11-13]. Liver resection combined with ablation of metastases [14-16] and / or induction chemotherapy to reduce hepatic tumor size or tumor load may render unresectable metastases resectable or may help reduce the extent of liver resections [17]. Staged resections, with or without portal vein embolisation / ligation, can be used for a two-step clearance of liver metastases, to increase future remnant liver volume and to achieve a definitive R0 status. Expanding the indications and application of the aforementioned strategies has increased the number of patients becoming resectable [18]. The objective of the current study was to evaluate the postoperative clinical outcome and long-term survival in patients undergoing liver resection for colorectal cancer liver metastases based on limited versus extended indications.

\section{MATERIAL AND METHODS}

PATIENTS

A prospective database became operational in the Maastricht University Medical Centre HPB unit in 2001. All patients that underwent liver surgery before this date were includ- 
ed retrospectively. Patients undergoing liver surgery for CRCLM between 1991 and 2010 were included in the present study and assigned to a group with limited indication criteria or a group with extended indication criteria for resection. Patients were staged by 4-phase contrast enhanced abdominal CT scan. All patients with either primary or secondary liver tumors were discussed at a multidisciplinary liver meeting. Patient specific co-morbidities and diagnostic procedures were assessed and the definitive treatment strategy was decided in consensus. Induction chemotherapy in irresectable patients, liver resection combined with tumor ablation, preoperative portal vein embolization, liver first policy in rectal cancer and repeat hepatectomy were all among potential surgical strategies. Obviously some of these strategies became available only in more recent years. Vascular reconstructions were occasionally used. In recent years, patients not undergoing liver surgery were assigned to stereotactic radiotherapy, percutaneous tumor ablation, Y90- selective internal radiotherapy or palliative chemotherapy.

\section{STUDY GROUPS}

Preoperative CT-, MRI- or PET-CT-scans were used to determine number and location of liver metastases. Operation notes gave insight in the specific type of resection, relation of metastases to the transection line, duration of surgery, the amount of blood loss and complications during surgery. Patients were, using predefined criteria, retrospectively assigned to either the extended indications group or to the limited indications group. Criteria for extended indications are depicted in table 1. Only patients with colorectal cancer liver metastases and a follow up of at least 6 months were included in the current study.

\begin{tabular}{|c|c|}
\hline Limited indication criteria & Extended indication criteria \\
\hline 1. Three or less liver metastases & 1. Four or more liver metastases \\
\hline 2. Located at one side of liver only & 2. Bilateral metastases \\
\hline 3. No signs of extra hepatic metastases & $\begin{array}{l}\text { 3. Presence of resectable extra } \\
\text { hepatic metastases }\end{array}$ \\
\hline $\begin{array}{l}\text { 4. Anticipated resection margin more } \\
\text { than } 10 \mathrm{~mm} \text {. }\end{array}$ & 4. Centrally located metastases \\
\hline
\end{tabular}

Table 1. Indication criteria for resection of colorectal liver metastases

\section{LIVER RESECTIONS}

All liver resections were classified in accordance with the IHPBA Brisbane nomenclature (table 2) [19]. Liver resection was performed as described before [20]. To determine the definitive extent of hepatic metastases and transection line, intra-operative ultrasound was used routinely.

\section{PERI-OPERATIVE CARE}

In February 2005 the Enhanced Recovery After Surgery (ERAS ${ }^{\circledR}$ ) fast track peri-operative care programme was introduced in liver surgery at our centre. This programme enhances 
postoperative recovery and as consequence reduces hospital length of stay [21]. Before the introduction of the ERAS programme there was no standard perioperative care protocol.

\section{ONCOLOGICALFOLLOW UP}

Follow up consisted of outpatient visits with plasma CEA levels, every three months and liver imaging twice in the first 2 years and annually up to 5 years after surgery. Median follow up was 33 months (range, 0-235). In case of recurrence, patients were assessed with PET-CT and the indication for repeat liver or lung surgery was discussed in the multidisciplinary oncology meeting.

\section{OUTCOME PARAMETERS}

The primary endpoint of the study was overall survival. Secondary endpoints were all complications, a liver surgery specific composite endpoint [22], readmissions, postoperative mortality, hospital length of stay (LOS) and disease free survival. Complications were registered daily using National Surgical Adverse Event Registration (LHCR) software [23] of the Dutch Association of General Surgery before 2009 and in the hospital information system (SAP, Walldorf, Germany) afterwards. Post operative course of all discharged patients was discussed at the morning surgeons meeting and the Dindo-Clavien classification was used to grade complications after surgery [24]. Complications with a Dindo-Clavien score $\leq 2$ were considered minor complications while complications $\geq 3$ a were considered major complications. Patient demographics were also registered and information on co-morbidity, location and TNM stage of the primary tumor and moment of occurrence of liver metastases were retrieved from patient charts. Size of the metastases and the resection margins were retrieved from pathology reports. R0 resections were defined as resections with a tumor-free resection-surface. If invasion in the resection-surface was present, the resection was considered $R 1$. $R 1$ resections with metastases reaching the resection surface were analyzed separately for local recurrences and survival.

Overall survival and disease free survival after liver resection were registered in months and calculated in percentages. Overall survival was calculated as the time period between the date of diagnosis and death. In case of two-stage hepatectomies, disease free survival was calculated as the time period between the first stage operation and the recurrence of metastases after the second stage operation.

\section{STATISTICAL ANALYSIS}

Data were analyzed using SPSS version 18.0 (SPSS Inc., Chicago, IL). Data are expressed as median and percentages or survival in months (95\% confidential interval). The Chi-square test was used to analyze categorical data while continuous data were analyzed using the t-test $U$ test. Time to recurrence and overall survival were calculated with the Kaplan-Meijer (sensored) method using the date of liver surgery and date of diagnosis respectively as reference date. A level of $p<0.05$ was considered to be statistically significant. 


\begin{tabular}{|c|c|c|c|c|}
\hline & $\begin{array}{l}\text { All patients } \\
\mathrm{N}=298\end{array}$ & $\begin{array}{l}\text { Limited Indication } \\
\text { criteria } \mathrm{N}=169\end{array}$ & $\begin{array}{l}\text { Extended Indication } \\
\text { criteria } \mathrm{N}=129\end{array}$ & $P$ \\
\hline \multicolumn{5}{|l|}{ Patient characteristics } \\
\hline Median age [range], y & $64[24-88]$ & $64[28-88]$ & $64[24-82]$ & 0.183 \\
\hline Sex, male $(\%)$ & $177(59.4)$ & $110(65.1)$ & $67(51.9)$ & 0.022 \\
\hline Co-morbidities, present (\%) & $100(33.6)$ & $56(33.1)$ & $44(34.1)$ & 0.860 \\
\hline \multicolumn{5}{|l|}{ ASA classification } \\
\hline Percentage with ASA 1 & 12.9 & 11.2 & 14.8 & 0.488 \\
\hline Percentage with ASA 2 & 60.8 & 59.2 & 62.5 & 0.604 \\
\hline Percentage with ASA 3 & 26.3 & 29.6 & 22.7 & 0.270 \\
\hline \multicolumn{5}{|l|}{ Primary tumor } \\
\hline Location, colon (\%) & $177(59.4)$ & $98(58.0)$ & $79(61.2)$ & 0.571 \\
\hline AJCC T-stage, T3/T4 disease (\%) & 87.9 & 84.8 & 92.0 & 0.082 \\
\hline Nodal status, positive nodes (\%) & 65.9 & 64.6 & 67.6 & 0.618 \\
\hline \multicolumn{5}{|l|}{ Hepatic metastases } \\
\hline Median size of largest lesion [range], $\mathrm{cm}$ & $3.0[0.0-20.0]$ & $3.0[0.4-13.0]$ & $3.0[0.0-20.0]$ & 0.877 \\
\hline Larger than $5 \mathrm{~cm}(\%)$ & 18.7 & 17.6 & 20.2 & 0.622 \\
\hline Median number [range] & $2[1-12]$ & $1[1-3]$ & $4[1-12]$ & $<0.001$ \\
\hline Bilateral metastases (\%) & $74(24.8)$ & 0 & $74(57.4)$ & - \\
\hline Concomitant extrahepatic disease (\%) & $30(10.1)$ & 0 & $30(23.3)$ & - \\
\hline \multicolumn{5}{|l|}{ Pre-operative management } \\
\hline Pre-operative chemotherapy (\%) & 42.1 & 34.5 & 52.0 & 0.003 \\
\hline \multicolumn{5}{|l|}{ Operative details } \\
\hline Two-stage procedure planned & 14 & 0 & 14 & - \\
\hline PVE during first stage & 9 & 0 & 9 & - \\
\hline Second stage performed & 10 & 0 & 10 & - \\
\hline Combined procedure (\%) & $13(4.2)$ & $7(4.1)$ & $6(4.3)$ & 0.940 \\
\hline Liver first procedure (\%) & $26(8.4)$ & $11(6.5)$ & $15(10.8)$ & 0.573 \\
\hline \multicolumn{5}{|l|}{ Type of Liver Resection (\%) } \\
\hline Major (> 3 segments) & $174(56.5)$ & $81(47.9)$ & $97(69.8)$ & $<0.001$ \\
\hline Left hemihepatectomy & $18(5.8)$ & $9(5.3)$ & $9(6.5)$ & 0.669 \\
\hline Right hemihepatectomy & $103(33.4)$ & $55(32.5)$ & $48(34.5)$ & 0.713 \\
\hline Left extended hemihepatectomy & $4(1.3)$ & $0(0.0)$ & $4(2.9)$ & - \\
\hline Right ext. hemihepatectomy & $12(3.9)$ & $2(1.2)$ & $10(7.2)$ & 0.007 \\
\hline Central liver resection & $15(4.9)$ & $0(0.0)$ & $15(10.8)$ & - \\
\hline $\begin{array}{l}\leq 1 \text { segmentectomy or } \\
\text { (multi) metastasectomy }\end{array}$ & $91(29.5)$ & $65(38.5)$ & $26(18.7)$ & $<0.001$ \\
\hline Multisegmentectomies ( $\geq 2$ ) & $64(20.8)$ & $38(22.5)$ & $26(18.7)$ & 0.416 \\
\hline Only PVE & $1(0.3)$ & $0(0.0)$ & $1(0.7)$ & - \\
\hline Concomitant local ablation* $(\%)$ & $10(3.2)$ & $1(0.6)$ & $9(6.7)$ & 0.004 \\
\hline \multicolumn{5}{|l|}{ Post-operative management } \\
\hline Post-operative chemotherapy (\%) & 37.2 & 36.6 & 37.8 & 0.841 \\
\hline
\end{tabular}




\section{RESULTS}

\section{PATIENTS}

298 patients who underwent 308 liver resections for CRCLM between 1991 and 2010 were included. Ten patients had a two-stage strategy. Explorations with irresectable disease at laparotomy $(n=6)$ were excluded for further analysis (figure 1 ). In all patients with repeat resections for recurrent CRCLM $(n=41)$ results were analyzed with the initial resection as reference. Median age was 65 years (range 24-88). A total of 169 patients were included in the limited indications group vs 129 patients in the extended indications group. Patient demographics, preoperative characteristics, intra-operative details and pathology details are presented in table 2 and 3. Co-morbidities were present in $33.6 \%$ of patients, predominantly cardiovascular disease (28.2\%), diabetes mellitus (7.7\%) and pulmonary disease $(4.0 \%)$. As expected through the allocation of patients, patients with extended indications had more extensive spread of metastatic disease as is evidenced by the number of metastases, number of patients with bilateral disease and with concomitant extrahepatic disease. These patients underwent extended hepatectomies more often compared with the limited indications group ( $10.1 \%$ vs $1.2 \%, \mathrm{p}<0.001)$. Surgical procedures are depicted in table 2 .

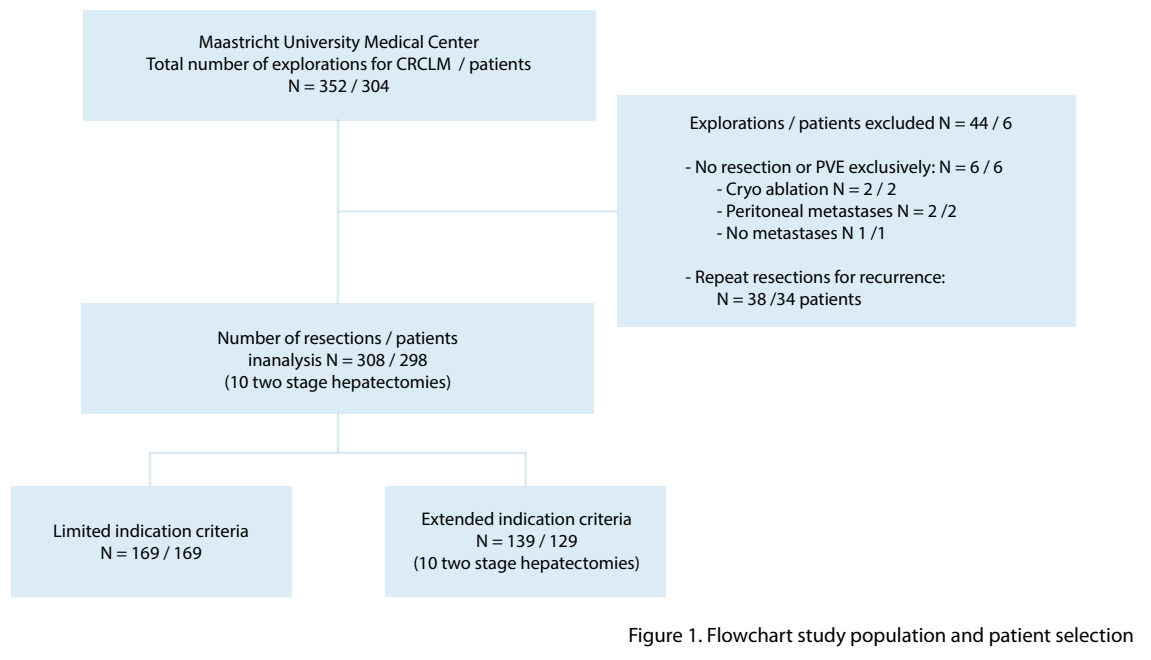

\section{PATHOLOGICAL ANALYSIS}

The percentage of $\mathrm{R} 0$ resections was $92.9 \%$ in the limited indications group and $77.5 \%$ in the extended indications group $(\mathrm{P}<0.001)$.

\section{SURGICAL OUTCOME}

There were no significant differences in blood loss during surgery, length of hospital stay and number of readmissions between both groups. However, there was a significant differ- 
ence in the median operation time 205 [75-660] minutes in the limited indications group vs 240 [85-660] minutes in the extended indications group $(p<0.001)$. Major complications occurred in $19.5 \%$ of the patients with limited indications versus $33.1 \%$ of the patients with extended indications ( $p=0.007$ ). 90 -day postoperative mortality rate was $3.6 \%$ in the limited indication group vs $5.0 \%$ in the extended indications group $(p=0.519)$. Outcome is depicted in table 3.

\begin{tabular}{|c|c|c|c|c|}
\hline & $\begin{array}{l}\text { All resections / patients } \\
\mathrm{N}=308 / 298\end{array}$ & $\begin{array}{l}\text { Limited indication criteria } \\
N=169 / 169\end{array}$ & $\begin{array}{l}\text { Extended indication } \\
\text { criteria } N=139 / 129\end{array}$ & $P$ \\
\hline \multicolumn{5}{|l|}{ Operative details } \\
\hline $\begin{array}{l}\text { Median estimated blood loss } \\
\text { [range], } \mathrm{mL}\end{array}$ & $800[10-11600]$ & $775[10-10000]$ & $800[50-11600]$ & 0.592 \\
\hline $\begin{array}{l}\text { Median duration of operation } \\
\text { [range], } \min \end{array}$ & $217[75-660]$ & $204.5[75-660]$ & $240[85-660]$ & $<0.001$ \\
\hline \multicolumn{5}{|l|}{ Pathology } \\
\hline \multicolumn{5}{|l|}{ Resection Margin, } \\
\hline Patients with $\mathrm{R} 0$ resections & $257(86.2)$ & $157(92.9)$ & $100(77.5)$ & $<0.001$ \\
\hline Patients with $\mathrm{R} 1$ resections & $41(13.8)$ & $12(7.1)$ & $29(22.5)$ & $<0.001$ \\
\hline Patients with $\mathrm{R} 2$ resections & 0.0 & 0.0 & 0.0 & - \\
\hline \multicolumn{5}{|l|}{ Length of hospital stay } \\
\hline $\begin{array}{l}\text { Median length of hospital stay } \\
\text { [range], days }\end{array}$ & $8[2-120]$ & 8 [3-92] & $8[2-120]$ & 0.579 \\
\hline \multicolumn{5}{|l|}{ Readmissions } \\
\hline $\begin{array}{l}\text { Number of patients readmit- } \\
\text { ted }(\%)\end{array}$ & $30(9.7)$ & $14(8.3)$ & $16(11.5)$ & 0.342 \\
\hline \multicolumn{5}{|l|}{ Repeat resections } \\
\hline $\begin{array}{l}\text { Number of patients under- } \\
\text { going repeat resections for } \\
\text { recurrence }(\%)\end{array}$ & $33(11.1)$ & $21(12.4)$ & $12(9.3)$ & 0.190 \\
\hline $\begin{array}{l}\text { Number of patients with } \\
\text { recurrence without resections } \\
\text { (\%) }\end{array}$ & $122(40.6)$ & $62(36.7)$ & $60(46.5)$ & 0.190 \\
\hline
\end{tabular}

Table 3. Comparison of surgical details, pathological details and LOS 


\section{OVERALL SURVIVAL}

Median overall survival for all patients was 55.9 months $(\mathrm{Cl}$ 48.5-63.3) with a 5-year overall survival rate of $48.3 \%$. Patients in the limited indications group had a median overall survival of 68.8 months $(\mathrm{Cl} 46.5-91.1)$ with a 5 year overall survival rate of $60.5 \%$. Patients in the extended indications criteria group had a median overall survival of 41.2 months $(\mathrm{Cl} 33.4-$ 49.0 ) and a 5 -year survival of $33.2 \%(p<0.001)$. 10-year overall survival rates in the limited indications group and the extended indications group were $35.5 \%$ and $15.8 \%$, respectively.

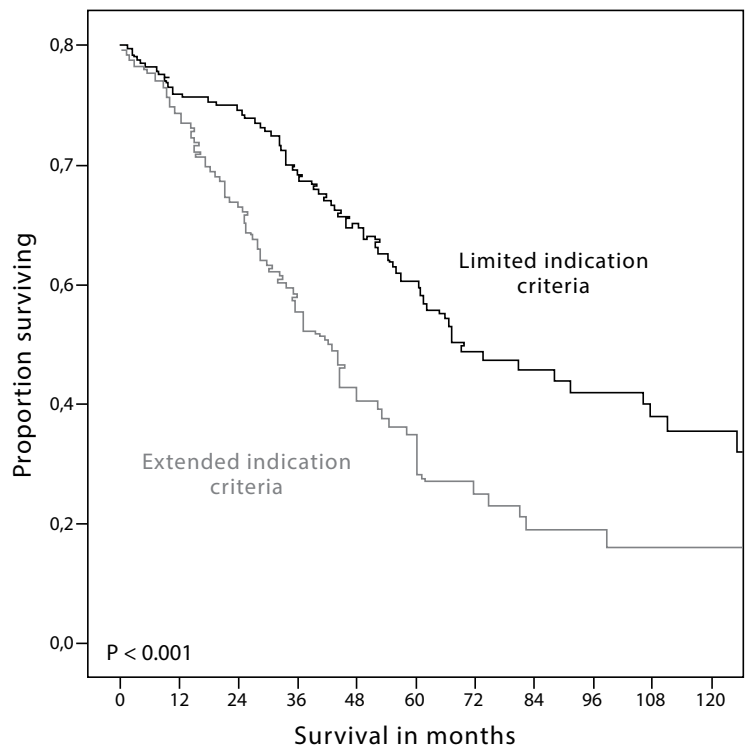

Number of patients at risk

Limited criteria

169

$137 \quad 114 \quad 86$

$\begin{array}{lllllll}3 & 42 & 32 & 25 & 20 & 13 & 12\end{array}$

Extended criteria

$129 \quad 112 \quad 83$

$83 \quad 56$

$56 \quad 32$

21

13

7

6

Figure 2. Overall survival

\section{5-DAY MORTALITY}

During the first postoperative year a steep drop in survival is observed in the Kaplan Meier curve in both groups (figure 2). We selected a group of all patients that died within 1 year after partial hepatectomy and compared multiple patient characteristics with a group of all patients that survived at least 1 year. The only significant difference between the group that died within 1 year and the group that survived at least 1 year was age (median 67.7 [28.686.1] years and 63.7 [24.2-88.2], respectively and $p=0.025$ ). Three more factors were borderline significant: combined oncological procedures, primary tumour positive lymph nodes and ASA 3. 9.5\% of the patients that died within 1 year had a liver resection combined with 
resection of the primary tumour, compared to $3.6 \%$ of the patients that survived at least 1 year, $\mathrm{p}=0.077$. Moreover, $77.5 \%$ of the patients that died within 1 year had primary colorectal positive lymph nodes compared to $63.7 \%$ of the patients surviving at least 1 year, $p=0.091$. Finally, $44.4 \%$ of the patients that died within 1 year were considered ASA 3 while only $24.6 \%$ of the patients that survived at least 1 year were considered as ASA 3, $p=0.069$.

\section{DISEASE FREE SURVIVAL}

Median disease free survival (DFS) for all patients was 15.7 months (CI 12.8-18.7) with a 5 -year DFS rate of $23.7 \%$. Patients in the limited indications group had a median DFS of 22.0 months ( $\mathrm{Cl}$ 15.8-28.2) and a 5-year DFS rate of 30.5\%. Patients in the extended indications group had a median DFS of 10.2 months $(\mathrm{Cl}$ 8.4-11.9) and a 5-year DFS rate of $14.2 \%$ $(p<0.001)$. Kaplan Meier curves of both overall and disease free survival are presented in figure 2 and figure 3.33 patients with hepatic recurrence underwent a repeat liver resection. $14 \%$ of these patients did not suffer a recurrence during follow up. $86 \%$ of patients, $82 \%$ in the limited indications group and $92 \%$ in the extended group, developed a second recurrence. Median time from the repeat resection to the second recurrence was 8.7 [0.0-18.9] months and 6.9 [1.9-11.9] months $(p=0.390)$ in both groups respectively.

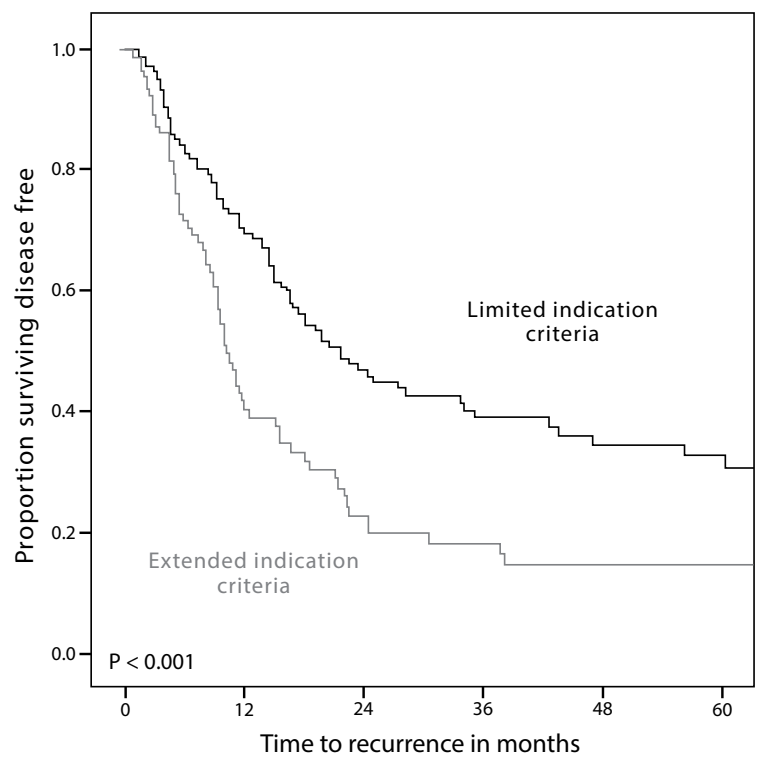

\begin{tabular}{|c|c|c|c|c|c|c|}
\hline \multicolumn{7}{|c|}{ Number of patients at risk } \\
\hline Limited criteria & 169 & 81 & 45 & 30 & 24 & 16 \\
\hline Extended criteria & 129 & 32 & 15 & 10 & 5 & 5 \\
\hline
\end{tabular}




\section{RECURRENCE AFTER R1 LIVER RESECTIONS}

On histological examination the resection specimen of 41 patients (13.3\%) showed a microscopically positive resection margin ( $\mathrm{R} 1$ resection). In these patients, recurrence of metastases in any location (i.e. liver and elsewhere) occurred in 23 (56\%) of 41 patients. Thirteen (32\%) of these patients developed hepatic recurrence of which $3(7 \%)$ had both intra- and extrahepatic recurrence. Only 7 (17\%) of patients with an R1 resection recurred at the former R1 resection surface. 1 patient in the limited indications group and 6 in the extended indications group. Ten (24\%) patients showed extra hepatic recurrence only. Eighteen (44\%) patients with an $\mathrm{R} 1$ resection stayed disease free during the follow up period (median follow up was 33.3 [0.2-165.9] months) (figure 4). Overall survival in all patients with an R0 resection was 60.0 [53.0-67.0] months with a 5-year survival rate of 50.4\%. Patients with an R1 resection showed a median overall survival of 44.3 [26.3-62.4] months and a 5-year survival rate of $34.9 \%(p=0.040)$. DFS in all patients with an R0 resection was 16.9 [13.9-19.9] months (5-year DFS rate 25.2\%) versus 9.4 [3.9-15.0] months (5-year DFS rate 17.4\%) in patients with an $\mathrm{R} 1$ resection $(\mathrm{p}=0.091)$.

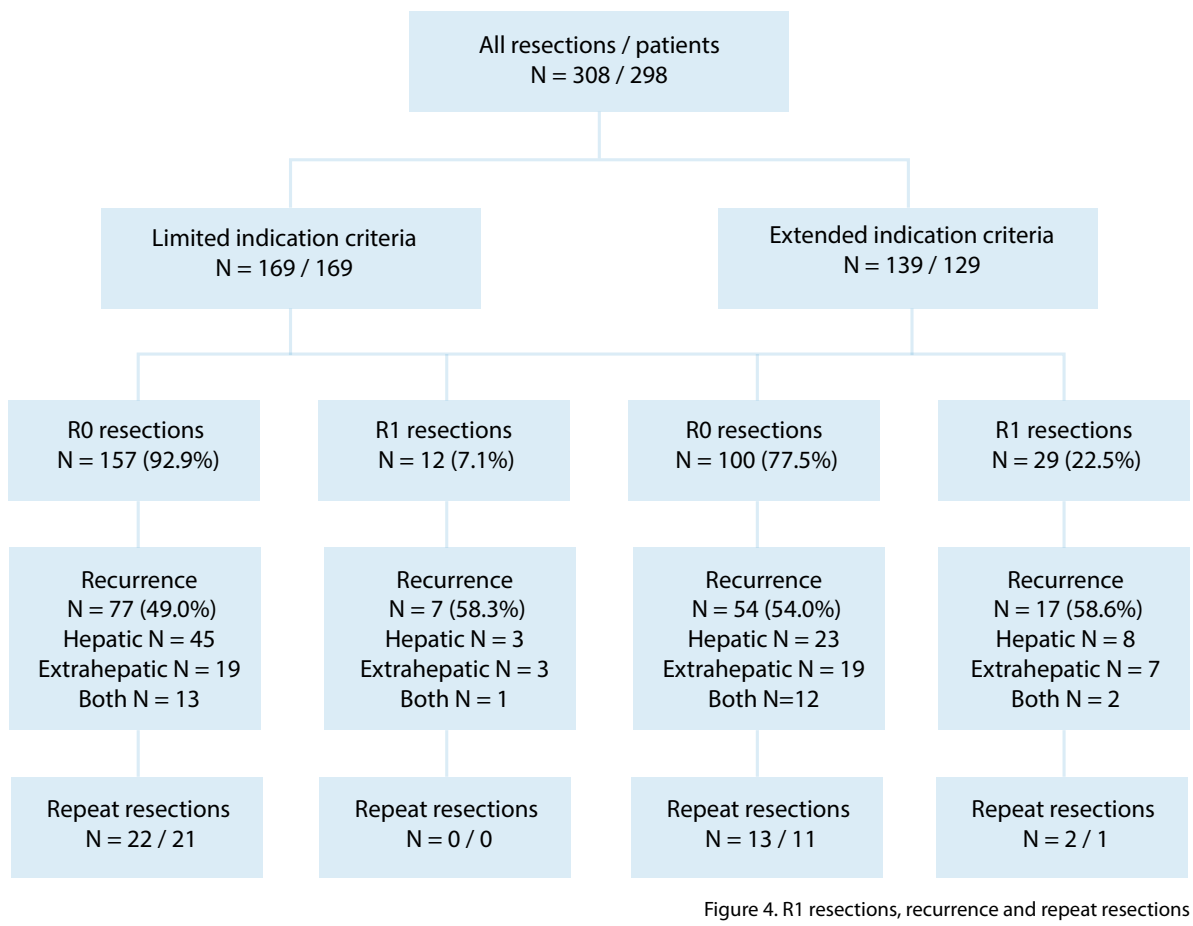




\begin{tabular}{|c|c|c|c|c|}
\hline & $\begin{array}{l}\text { All resections / } \\
\text { patients } \\
\mathrm{N}=308 / 298\end{array}$ & $\begin{array}{l}\text { Limited indication } \\
\text { criteria } \\
N=169 / 169\end{array}$ & $\begin{array}{l}\text { Extended indication } \\
\text { criteria } \\
\mathrm{N}=139 / 129\end{array}$ & $\mathrm{P}$ \\
\hline \multicolumn{5}{|l|}{ Complications } \\
\hline Complications & $113(36.7)$ & $54(32.0)$ & $59(42.4)$ & 0.057 \\
\hline Minor complications & $34(11.0)$ & $21(12.4)$ & $13(9.4)$ & 0.392 \\
\hline Dindo-Clavien grade I & $10(3.2)$ & $5(3.0)$ & $5(3.6)$ & 0.753 \\
\hline Dindo-Clavien grade II & $24(7.8)$ & $16(9.5)$ & $8(5.8)$ & 0.227 \\
\hline Major complications & $79(25.6)$ & $33(19.5)$ & $46(33.1)$ & 0.007 \\
\hline Dindo-Clavien grade Illa & $48(15.6)$ & $20(11.8)$ & $28(20.1)$ & 0.045 \\
\hline Dindo-Clavien grade IIIb & $8(2.6)$ & $2(1.2)$ & $6(4.3)$ & 0.085 \\
\hline Dindo-Clavien grade IVa & $10(3.2)$ & $5(3.0)$ & $5(3.6)$ & 0.753 \\
\hline Dindo-Clavien grade IVb & $0(0.0)$ & $0(0.0)$ & $0(0.0)$ & - \\
\hline Dindo-Clavien grade $\mathrm{V}$ & $13(4.2)$ & $6(3.6)$ & $7(5.0)$ & 0.519 \\
\hline \multicolumn{5}{|c|}{ Liver surgery specific composite endpoint (CEP) } \\
\hline Liver surgery-specific CEP & $67(21.8)$ & $29(17.2)$ & $38(27.3)$ & 0.031 \\
\hline Ascites & $8(2.6)$ & $2(1.2)$ & $6(4.3)$ & 0.085 \\
\hline Postresectional liver failure & $11(3.6)$ & $4(2.4)$ & $7(5.0)$ & 0.209 \\
\hline Bile leakage & $16(5.2)$ & $5(3.0)$ & $11(7.9)$ & 0.051 \\
\hline Intra-abdominal haemorrhage & $9(2.9)$ & $4(2.4)$ & $5(3.6)$ & 0.524 \\
\hline Intra-abdominal abscess & $35(11.4)$ & $19(11.2)$ & $16(11.5)$ & 0.941 \\
\hline 90 day postoperative mortality & $13(4.2)$ & $6(3.6)$ & $7(5.0)$ & 0.519 \\
\hline \multicolumn{5}{|l|}{ Other liver related complications } \\
\hline Hepatic encephalopathy & $5(1.6)$ & $2(1.2)$ & $3(2.2)$ & - \\
\hline Liver dysfunction & $2(0.6)$ & $0(0.0)$ & $2(1.4)$ & - \\
\hline Cholangitis & $2(0.6)$ & $1(0.6)$ & $1(0.7)$ & - \\
\hline \multicolumn{5}{|l|}{ Other complications } \\
\hline Sepsis & $9(2.9)$ & $5(3.0)$ & $4(2.9)$ & 0.943 \\
\hline Cardiovascular & $13(4.2)$ & $8(4.7)$ & $5(3.6)$ & 0.719 \\
\hline Pulmonary & $28(9.1)$ & $17(10.1)$ & $11(7.9)$ & 0.653 \\
\hline Renal / urine tract & $12(3.9)$ & $8(4.7)$ & $4(2.9)$ & 0.477 \\
\hline Gastro-intestinal & $18(5.8)$ & $7(4.1)$ & $11(7.9)$ & 0.115 \\
\hline Hematologic & $1(0.3)$ & $0(0.0)$ & $1(0.7)$ & - \\
\hline Neurologic & $9(2.9)$ & $1(0.6)$ & $8(5.8)$ & - \\
\hline Wound infection & $7(2.3)$ & $3(1.8)$ & $4(2.9)$ & - \\
\hline
\end{tabular}




\section{DISCUSSION}

In this study, short- and long-term outcome in patients undergoing liver resection for colorectal cancer liver metastases with extended indications, were assessed and compared with a group of patients operated according to limited indications criteria. We showed in the extended indications group a 5 -yr survival of $33.2 \%$ equivalent to a median overall survival of 41 months. In patients undergoing liver resection according to the limited indications a 5 -year overall survival of $60.5 \%$ was observed, equivalent to a median overall survival of 69 months. In the latter group 5-year disease free survival was $30.5 \%$ equivalent with a median disease free survival of 22 months. In patients with extended indications 5-year disease free survival was $14.2 \%$ equivalent with a median disease free survival of 10 months, which is significantly lower than in patients with limited indications. When patients underwent a repeat liver resection for recurrence median disease free survival was prolonged with 8 months. Although outcome was worse in the extended indication group, cure, defined as disease free survival longer than 10 years, was observed in $15.8 \%$ of patients.

Recently, short and long-term outcomes in patients undergoing liver resection for colorectal cancer liver metastases before and after 2000 have been published [25]. In that study two time periods with mixed indication criteria were compared. Although extended indication criteria were used in the patient groups before and after 2000, most patients operated after 2000 were still treated within the limited indication criteria. This may explain, the relatively small difference observed in 5 -year overall survival of $47 \%$ and $58 \%$ between the two groups, respectively in that study. Outcome results regarding patients undergoing liver resection for colorectal cancer liver metastases within extended indication criteria are scarce. Recent publications report on the influence on outcome of only one single component of the former contraindications, e.g. number of metastases, resection margin or extra hepatic disease $[4,5,7,26]$.

The results of our study are in line with recent publications and are slightly better than the results of a recently published meta-analysis of studies on liver resection for colorectal cancer liver metastases before 2007 [10, 25, 27, 28]. From most published series it transpires that over time survival after liver resection of CRCLM improves, probably as a consequence of improved effectiveness of modern chemotherapy regimens, improved perioperative care, better patient selection and more aggressive resections. The overall survival of 56 months in our study may be also a consequence of the long timeframe of this study and the influence of modern chemotherapy regimens as well as additional treatment strategies in both patient groups with limited and extended indication criteria and seems to support an aggressive strategy towards CRCLM. From clinical practice we observe that extending the indications for liver resection of colorectal cancer liver metastases renders more patients eligible. Our data show that extending the indications has its consequences for patients. It leads to more major complications, a shorter overall survival and a shorter disease free survival 
compared to patients with less extensive metastases. Major complications often require surgical, endoscopic, or radiological interventions. Although it may not be entirely legitimate to compare the median overall survival of 41 months in patients eligible for liver resection within extended indications to the median survival of 18-22 months in patients treated with chemotherapy alone, survival after liver resection probably is substantially longer [29-32]. The increase in overall survival of approximately one and a half year compared to the available literature data on outcome after palliative chemotherapy alone, seems to justify the acceptance of a higher complication rate after resection. Wiering et al. showed that quality of life recovered to baseline within three months in patients undergoing potentially curative liver resection while a persistent decline in quality of life was demonstrated in patients immediately treated with palliative chemotherapy alone [33]. If patients turned out to be irresectable at laparotomy, quality of life was worst [34]. It can therefore be hypothesized that the occurrence of complications after liver surgery with curative intent is an acceptable phenomenon for patients. Futile laparotomies should be avoided by optimal preoperative patient selection.

The morbidity and mortality results in the present study are consistent with other recently published series [25,35], although comparison is hampered to some extent since definitions used for severe or major morbidity vary across studies. In addition to standardized definitions of complications, the use of a composite endpoint for liver surgery specific complications could enhance comparability of published series [22].

Strikingly, the occurrence of more severe complications did not prolong hospital length of stay and did not lead to more readmissions. It can be hypothesized that the expected prolonged hospital length of stay as a consequence of a higher percentage of major morbidity was compensated by the introduction of the Enhanced Recovery after Surgery (ERAS ${ }^{\circledR}$ ) programme in our unit in 2005. A substantial proportion of patients deceased in the first year after partial liver resection in both the extended indications and the limited indications group. In this study, high age, ASA 3 and combined liver and colon resections showed to be risk factors for a high 365-day mortality. Hence, it should be possible to reduce postoperative mortality with better preoperative patient selection. Unfortunately, our data, as well as other published data did not show predictive factors usable to select patients who were at risk for mortality and consequently did not benefit from surgery [36, 37].

Compared to patients with limited indications, patients with extended indications underwent more often an $\mathrm{R} 1$ resection. In this series this did not lead to more repeat resections in the latter group. Moreover, we showed that only $17 \%$ of the patients with a R1 resection recurred at the level of the pathologically irradical resection surface. This might be a result of the use of the CUSA device (Integra LifeSiences, Plainsboro, NJ, USA), which prevents the pathologist to evaluate the actual resection surface, as approximately 1 to 3 millimeters of hepatocytes disintegrate with the use of this device. The consequence of this observation 
might be that a close relation of metastases to vascular structures that cannot be resected is not an absolute contraindication for resection. Moreover, an R0 resection rate of $77.5 \%$ and a median survival of 41 months as well as the cure percentage of $15.8 \%$, seems to justify the use of extended indication criteria. As others have provided data to suggest these patients have a good quality of life, it also seems justified to be creative in future strategies to increase the resection rate in patients with colorectal cancer liver metastases. Suggestions might be to combine different treatment modalities that aim to remove or destroy metastases while functional liver capacity is maintained or enhanced. Open- or laparoscopic-resections combined with ablation, irreversible electroporation, perioperative stereotactic radiotherapy or selective Internal Radio Therapy (SIRT) with Ytrium-bound microspheres [38], may help to increase the Ro resection rate and further improve survival with acceptable quality of life.

The new technique of in situ liver partition and portal vein ligation for 2-stage hepatectomy with a short interval (ISLT or ALPPS) seems promising but the current morbidity and mortality of this procedure is still too high [39-41].

Strong points of this study are the selection of a series of patients with extended indication criteria and a standardized complication registration. Limitations are the relatively small sample size due to the single centre study design, the large time period during which the were patients included, the different perioperative neo-adjuvant or adjuvant chemotherapy strategies and the retrospective analysis of prospectively collected outcome data.

\section{CONCLUSION}

In the present study liver resection for colorectal cancer liver metastases with extended indication criteria had an $\mathrm{R} 0$ resection rate of $77.5 \%$, resulted in a median 5 -year overall survival of over $33.2 \%$ and cure in $15.8 \%$ of patients with acceptable postoperative mortality and morbidity. 


\section{REFERENCES}

1. Fong, Y., et al., Clinical score for predicting recurrence after hepatic resection for metastatic colorectal cancer: analysis of 1001 consecutive cases. Ann Surg, 1999. 230(3): p. 309-18; discussion 318-21.

2. Nordlinger, B., et al., Surgical resection of colorectal carcinoma metastases to the liver. A prognostic scoring system to improve case selection, based on 1568 patients. Association Francaise de Chirurgie. Cancer, 1996. 77(7): p. 1254-62.

3. Sharma, S., C. Camci, and N. Jabbour, Management of hepatic metastasis from colorectal cancers: an update. J Hepatobiliary Pancreat Surg, 2008. 15(6): p. 570-80.

4. Altendorf-Hofmann, A. and J. Scheele, A critical review of the major indicators of prognosis after resection of hepatic metastases from colorectal carcinoma. Surg Oncol Clin N Am, 2003. 12(1): p. 165-92, xi.

5. Pawlik, T.M., et al., Debunking dogma: surgery for four or more colorectal liver metastases is justified. J Gastrointest Surg, 2006. 10(2): p. 240-8.

6. Mehrabi, A., et al., Mesohepatectomy as an option for the treatment of central liver tumors. J Am Coll Surg, 2008. 207(4): p. 499-509.

7. Pulitano, C., et al., Liver resection for colorectal metastases in presence of extrahepatic disease: results from an international multi-institutional analysis. Ann Surg Oncol, 2011. 18(5): p. 1380-8.

8. Fong, Y., Surgical therapy of hepatic colorectal metastasis. CA Cancer J Clin, 1999. 49(4): p. 231-55.

9. Shimada, H., et al., Treatment for colorectal liver metastases: a review. Langenbecks Arch Surg, 2009. 394(6): p. 973-83.

10. Pawlik, T.M., R.D. Schulick, and M.A. Choti, Expanding criteria for resectability of colorectal liver metastases. Oncologist, 2008. 13(1): p. 51-64.

11. Adam, R., et al., Two-stage hepatectomy: A planned strategy to treat irresectable liver tumors. Ann Surg, 2000. 232(6): p. 777-85.

12. Gallagher, D.J. and N. Kemeny, Metastatic colorectal cancer: from improved survival to potential cure. Oncology, 2010. 78(3-4): p. 237-48.

13. Yang, A.D., A. Brouquet, and J.N. Vauthey, Extending limits of resection for metastatic colorectal cancer: risk benefit ratio. J Surg Oncol, 2010. 102(8): p. 996-1001.

14. Evrard, S., et al., Unresectable colorectal cancer liver metastases treated by intraoperative radiofrequency ablation with or without resection. Br J Surg, 2012. 99(4): p. 558-65.

15. Razafindratsira, T., M. Isambert, and S. Evrard, Complications of intraoperative radiofrequency ablation of liver metastases. HPB (Oxford), 2011. 13(1): p. 15-23.

16. Ruers, T., et al., Radiofrequency ablation combined with systemic treatment versus systemic treatment alone in patients with non-resectable colorectal liver metastases: a randomized EORTC Intergroup phase II study (EORTC 40004). Ann Oncol, 2012. 23(10): p. 2619-2626.

17. Folprecht, G., et al., Tumour response and secondary resectability of colorectal liver metastases following neoadjuvant chemotherapy with cetuximab: the CELIM randomised phase 2 trial. Lancet Oncol, 2010. 11(1): p. 38-47. 18. Lam, V.W., et al., A systematic review of clinical response and survival outcomes of downsizing systemic chemotherapy and rescue liver surgery in patients with initially unresectable colorectal liver metastases. Ann Surg Oncol, 2012. 19(4): p. 1292-301.

19. Strasberg, S.M., Nomenclature of hepatic anatomy and resections: a review of the Brisbane 2000 system. J Hepatobiliary Pancreat Surg, 2005. 12(5): p. 351-5.

20. van de Poll, M.C., et al., Interorgan amino acid exchange in humans: consequences for arginine and citrulline metabolism. Am J Clin Nutr, 2007. 85(1): p. 167-72.

21. van Dam, R.M., et al., Initial experience with a multimodal enhanced recovery programme in patients undergoing liver resection. Br J Surg, 2008. 95(8): p. 969-75. 22. van den Broek, M.A., et al., Development of a composite endpoint for randomized controlled trials in liver surgery. Br J Surg, 2011. 98(8): p. 1138-45.

23. Marang-van de Mheen, P.J., M.C. Stadlander, and J. Kievit, Adverse outcomes in surgical patients: implemen- 
tation of a nationwide reporting system. Qual Saf Health Care, 2006. 15(5): p. 320-4.

24. Dindo, D., N. Demartines, and P.A. Clavien, Classification of surgical complications: a new proposal with evaluation in a cohort of 6336 patients and results of a survey. Ann Surg, 2004. 240(2): p. 205-13.

25. de Haas, R.J., et al., Impact of expanding criteria for resectability of colorectal metastases on short- and longterm outcomes after hepatic resection. Ann Surg, 2011. 253(6): p. 1069-79.

26. Pawlik, T.M., et al., Effect of surgical margin status on survival and site of recurrence after hepatic resection for colorectal metastases. Ann Surg, 2005. 241(5): p. 715-22, discussion 722-4.

27. de Jong, M.C., et al., Rates and patterns of recurrence following curative intent surgery for colorectal liver metastasis: an international multi-institutional analysis of 1669 patients. Ann Surg, 2009. 250(3): p. 440-8.

28. Kanas, G.P., et al., Survival after liver resection in metastatic colorectal cancer: review and meta-analysis of prognostic factors. Clin Epidemiol, 2012. 4: p. 283-301. 29. Cassidy, J., et al., XELOX vs FOLFOX-4 as first-line therapy for metastatic colorectal cancer: NO16966 updated results. Br J Cancer, 2011. 105(1): p. 58-64.

30. Maughan, T.S., et al., Addition of cetuximab to oxaliplatin-based first-line combination chemotherapy for treatment of advanced colorectal cancer: results of the randomised phase 3 MRC COIN trial. Lancet, 2011. 377(9783): p. 2103-14.

31. Saltz, L.B., et al., Bevacizumab in combination with oxaliplatin-based chemotherapy as first-line therapy in metastatic colorectal cancer: a randomized phase III study. J Clin Oncol, 2008. 26(12): p. 2013-9.

32. van lersel, L.B., et al., Management of isolated nonresectable liver metastases in colorectal cancer patients: a case-control study of isolated hepatic perfusion with melphalan versus systemic chemotherapy. Ann Oncol, 2010. 21(8): p. 1662-7.

33. Wiering, B., et al., Long-term global quality of life in patients treated for colorectal liver metastases. Br J Surg,
2011. 98(4): p. 565-71; discussion 571-2.

34. Langenhoff, B.S., et al., Quality of life after surgical treatment of colorectal liver metastases. Br J Surg, 2006. 93(8): p. 1007-14.

35. Farges, O., et al., Incidence and risks of liver resection: an all-inclusive French nationwide study. Ann Surg, 2012. 256(5): p. 697-704; discussion 704-5.

36. Abbott, A.M., et al., Short-term outcomes after combined colon and liver resection for synchronous colon cancer liver metastases: a population study. Ann Surg Oncol, 2013. 20(1): p. 139-47.

37. Schiesser, M., et al., Perioperative morbidity affects long-term survival in patients following liver resection for colorectal metastases. J Gastrointest Surg, 2008. 12(6): p. 1054-60.

38. Vente, M.A., et al., Yttrium-90 microsphere radioembolization for the treatment of liver malignancies: a structured meta-analysis. Eur Radiol, 2009. 19(4): p. 951-9.

39. Knoefel, W.T., et al., In situ liver transection with portal vein ligation for rapid growth of the future liver remnant in two-stage liver resection. Br J Surg, 2013. 100(3): p. 388-94.

40. Neumann, U.P. and C.H. Dejong, Split decision. Br J Surg, 2013. 100(3): p. 310-2.

41. Schnitzbauer, A.A., et al., Right portal vein ligation combined with in situ splitting induces rapid left lateral liver lobe hypertrophy enabling 2-staged extended right hepatic resection in small-for-size settings. Ann Surg, 2012. 255(3): p. 405-14. 


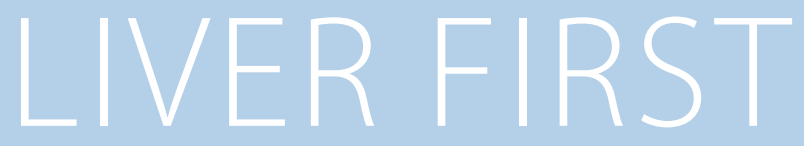

\section{PUBLISHED AS}

The liver-first approach for synchronous colorectal liver metastasis: a 5-year single-centre experience

M.C. de Jong, R.M. van Dam M. Maas, M.H.A. Bemelmans, S.W.M. Olde Damink, G.L. Beets, C.H.C. Dejong

HPB 2011, 13(10) 745-752

DOI: dx.doi.org/10.1111/j.1477-2574.2011.00372.x 


\section{ABSTRACT}

\section{BACKGROUND}

For patients who present with synchronous colorectal carcinoma and colorectal liver metastasis (CRLM), a reversed treatment sequence in which the CRLM are resected before the primary carcinoma has been proposed (liver-first approach). The aim of the present study was to assess the feasibility and outcome of this approach for synchronous CRLM.

\section{METHODS}

Between 2005 and 2010, 22 patients were planned to undergo the liver-first approach. Feasibility and outcomes were prospectively evaluated.

\section{RESULTS}

Of the 22 patients planned to undergo the liver-first strategy, the approach was completed in 18 patients $(81.8 \%)$. The main reason for treatment failure was disease progression. Patients who completed treatment and patients who deviated from the protocol had a similar location of the primary tumour, as well as comparable size, number and distribution of CRLM (all P > 0.05). Post-operative morbidity and mortality were $27.3 \%$ and $0 \%$ following liver resection and $44.4 \%$ and $5.6 \%$ after colorectal surgery, respectively. On an intention-to-treat-basis, overall 3-year survival was $41.1 \%$. However, $37.5 \%$ of patients who completed the treatment had developed recurrent disease at the time of the last follow-up.

\section{CONCLUSION}

The liver-first approach is feasible in approximately four-fifths of patients and can be performed with peri-operative mortality and morbidity similar to the traditional treatment paradigm. Patients treated with this novel strategy derive a considerable overall-survival-benefit, although disease recurrence-rates remain relatively high, necessitating a multidisciplinary approach. 


\section{INTRODUCTION}

Up to one-fourth of patients who present with colorectal cancer have concomitant colorectal liver metastasis (CRLM)[1, 2]. Patients with these synchronous CRLM are thought to have a worse prognosis compared with patients with metachronous CRLM[3].3 While surgical resection of CRLM is regarded the only potential for a cure, the simultaneous presentation of primary and secondary disease provides a unique chance in deciding the optimal therapy sequence in these patients[4,5].

The classic approach for patients with synchronous CRLM encompasses resection of the primary tumour, followed by optional adjuvant chemotherapy and eventually succeeded by liver surgery. Potential advantages of this approach are primary tumour oriented and include prevention of local ingrowth, bowel obstruction or bleeding from the colorectal carcinoma. However, major disadvantages include progression of the CRLM beyond resectability, especially after a delay in the treatment paradigm owing to morbidity associated with the colorectal surgery. Moreover, in case of chemotherapy, serious hepatoxicity can occur or the hepatic lesions can disappear[6-8]. While some authors have therefore supported a simultaneous resection of the primary tumour and concurrent liver disease[9-11], others advocated a staged approach in which the liver is operated on before the primary tumour and increasingly frequently preceded by induction chemotherapy[12-14]. Particularly for rectal primaries, the latter approach is regarded as more natural as the post-radiation required waiting period offers ample time for performance of a liver resection. Additional rationale for this reversed sequence of therapy is that CRLM are the main cause of death and that it is therefore important to eliminate these lesions first. With a liver-first approach, the treatment of the CRLM is by no means interrupted by possible complications after resection of the primary tumour. A concurrent advantage could be that administration of pre-operative chemotherapy provides a chance to evaluate a response and thereby define the tumour biology of the CRLM. Moreover this strategy provides a certain window during which possibly latently present extrahepatic metastases have a chance to declare themselves $[15,16]$. Currently, there are very little data available regarding the liver-first approach $[12-14,17,18]$. Therefore, the aim of the present study was to describe the experience with the liver-first approach in a tertiary referral centre. Moreover, we sought to not only examine the feasibility and short-term outcomes after the liver-first approach, but also to assess the influence of this sequence in therapy on the oncological outcome.

\section{METHODS}

Prospectively collected data on patients who underwent liver surgery from 1st January 2005 to 31 st December 2010 were queried from the hepatectomy databases at a large hepatobil- 
iary centre (Maastricht University Medical Centre). All patients with synchronous CRLM were identified. In 22 of these patients, a liver-first approach was planned and these patients are the scope of the present study. During pre-operative assessment, patients were deemed resectable only if a resection with negative margins was expected for all known disease, both intra-and extrahepatic. Moreover, only patients in whom an adequate future liver remnant was anticipated on computed tomography (CT) volumetric analysis were considered candidates for hepatic resection[19, 20]. Furthermore, the future liver remnant had to incorporate a minimum of two adjacent segments with sufficient vascular in-and outflow and adequate biliary drainage to be regarded suitable for resection[5, 21].

In short, after patients were diagnosed with synchronous CRLM, they were referred to our tertiary referral centre (coordinated by the Surgical Oncologic Network of Limburg) and were subsequently presented and discussed in a multi-disciplinary oncology meeting, during which their treatment strategy was established. Specifically, all patients with rectal cancer underwent standard locoregional staging with standard T2-weighted magnetic resonance imaging (MRI) which was in some cases extended with an additional gadofosveset-enhanced imaging sequence for lymph node staging, in accordance with our institutional protocol described previously[22]. For patients with a primary tumour located in the colon, pre-operative staging was generally ascertained using a computed tomography (CT) scan. In general, all patients who were diagnosed with a rectal tumour, based on these imaging modalities, received pre-operative radiation therapy consisting of either a short course of 5 fractions of $5 \mathrm{~Gy}$ radiation or a long course of 28 fractions of $1.8 \mathrm{~Gy}$ radiation with capecitabine (chemoradiation, i.e. chemotherapy as a radiosensitizer, capecitabine 2x 1000 $\mathrm{mg} / \mathrm{m} 2$ ), followed by pre-operative chemotherapy aimed at minimizing the likelihood of a (microscopically) positive resection margin after resection of the primary tumour, according to the national guidelines[23]. In line with our institutional chemotherapy protocol, patients receive a total of six cycles. Moreover, in our institution, patients with a locally advanced rectal carcinoma, which was defined as a T4 tumour or a T3 tumour with involved or threatened mesorectal fascia or an N2-status or a distally located T3N1 tumour, receive chemoradiation followed by two cycles of pre-operative full-strength chemotherapy (i.e. oxaliplatin, $130 \mathrm{mg} /$ $\mathrm{m} 2$ with or without bevacizumab $7.5 \mathrm{mg} / \mathrm{kg}$ ). Patients with a non-locally advanced rectum carcinoma are generally treated with a short course of radiation therapy followed by three cycles of pre-operative chemotherapy (i.e. oxaliplatin, $130 \mathrm{mg} / \mathrm{m} 2$ with or without bevacizumab $7.5 \mathrm{mg} / \mathrm{kg}$ ). This also allowed evaluation of the tumour response to chemotherapy. The remaining cycles are generally administered either after liver resection or after completion of the resection of the primary tumour. Each deviation from this protocol only takes place after careful and thorough evaluation at our weekly, institutional multi-disciplinary meeting. Reasons for not completing all cycles of chemotherapy are mainly patient based and include too severe side-effects of pre-operative chemotherapy. However, rather than treating all patients according to a strict and rigid protocol, we intend to provide each pa- 
tient with a tailor-made treatment regimen.

After initial resectability of the CRLM was assessed based on the criteria described above, all patients with a primary tumour located in the rectum were considered for the liver-first approach, whereas patients with a colorectal tumour located in the colon were selected on a case-by-case basis in order to provide a tailor-made management plan.

\section{DATA COLLECTION}

Apart from standard demographic data (i.e. age and gender), the following data were also collected for each patient: characteristics of the primary tumour (i.e. location and size of the primary lesions, TNM stage) as well as of the CRLM (i.e. distribution, number and size of the lesions). Moreover, data concerning treatment-related variables were collected (i.e. peri-operative receipt of radiation and/or chemotherapy, details of the hepatic surgery as well as data regarding the operation on the primary tumour). Furthermore, data regarding post-operative outcome (i.e. presence, type and severity of in-hospital or 90-day morbidity and post-operative mortality within 90 days of treatment) and data regarding disease recurrence and vital status were noted. Disease recurrence was defined as a lesion that was a biopsy proven recurrent adenocarcinoma or a lesion that was deemed suspicious on cross-sectional imaging in the setting of an elevated carcinoembryonic antigen (CEA) level.

\section{STATISTICAL ANALYSES}

All statistical analyses were performed using SPSS version 17.0 (SPSS Inc., Chicago, IL, USA). Summary statistics were obtained and presented as percentages or median values. Upon comparing categorical data, the c2-test, or if deemed appropriate Fisher's exact test, was used, while the Mann-Whitney U-test was used to compare continuous data. Recurrence-free and overall survival analyses were performed using the non-parametric product limit method[24]. Overall, a P-value less than 0.05 was considered significant. 


\begin{tabular}{|c|c|}
\hline Variable & No. of patients (\%), $n=22$ \\
\hline \multicolumn{2}{|l|}{ Patient characteristics } \\
\hline Median age [range], y & $65[41-86]$ \\
\hline Sex (male) & $16(72.7)$ \\
\hline Median pre-operative CEA level [range], ug/L & $15.8[1.90-321.0]$ \\
\hline \multicolumn{2}{|l|}{ Primary Tumor Site } \\
\hline \multicolumn{2}{|l|}{ Location of primary tumor } \\
\hline Colon & $3(13.6)$ \\
\hline Rectum & 19 (86.4) \\
\hline \multicolumn{2}{|l|}{ Symptoms caused by primary tumor } \\
\hline \multicolumn{2}{|l|}{ Symptoms at time of presentation } \\
\hline None & $7(31.8)$ \\
\hline Rectal Blood Loss & $9(40.9)$ \\
\hline Changes in bowel habits & $4(18.2)$ \\
\hline Bowel obstruction & $3(13.6)$ \\
\hline \multicolumn{2}{|l|}{ Onset of symptoms during course of illness } \\
\hline None & $5(22.7)$ \\
\hline Bowel obstruction & $2(9.1)$ \\
\hline \multicolumn{2}{|l|}{ AJCC T-stage on pathology* } \\
\hline урт1/урт2 & $2(12.5)$ \\
\hline урT3/ypT4 & $14(87.5)$ \\
\hline \multicolumn{2}{|l|}{ Lymph node status on pathology* } \\
\hline ypN1/ypN2 & $6(37.5)$ \\
\hline ypNo & $10(62.5)$ \\
\hline \multicolumn{2}{|l|}{ Hepatic Metastasis } \\
\hline Size of largest metastasis (median [range]), $\mathrm{cm}$ & $1.7[0.4-6.6]$ \\
\hline No. of metastasis (median [range]) & $2[1-7]$ \\
\hline Location (unilobular) & $10(45.5)$ \\
\hline
\end{tabular}

Table 1 Patients and tumour characteristics 


\section{RESULTS}

\section{PATIENT AND TUMOUR CHARACTERISTICS}

Between 1st January 2005 and 31st December 2010, 186 patients underwent liver directed surgery for CRLM at our institution and were queried from our hepatobiliary database. Specifically, 90 patients presented with synchronous CRLM and in 22 of these patients (24.4\%), a liver-first approach was planned during this 5-year period. These latter patients are the scope of the present study. The characteristics of these patients are detailed in Table 1. The site of the primary tumour was the rectum in most patients $(n=19 ; 86.4 \%)$, whereas three patients (13.6\%) had a carcinoma located in the colon. The median pre-operative CEA level (range) was $15.8 \mathrm{mg} / \mathrm{L}$ (1.90-321.0).

At the time of presentation, 15 patients (68.2\%) complained of primary tumour-associated symptoms. Furthermore, another two patients (12.5\%) developed symptoms caused by the primary tumour during the course of their illness, but before their liver-directed surgery. Both of these patients developed an obstruction of the bowel as a result of their colorectal carcinoma and subsequently underwent a laparotomy during which a diverting ostomy was constructed, before their liver-directed surgery.

\section{DETAILS OF RADIATION AND CHEMOTHERAPEUTICTHERAPY}

In total, 21 patients $(95.5 \%)$ received radiation therapy of their primary tumour. Eight patients (36.4\%) received a short course of radiation therapy, of whom seven patients (31.8\%) also received multiple cycles of pre-operative chemotherapy before surgery. Moreover, 13 patients (59.1\%) underwent a long course of radiotherapy with a chemo-sensitizer (chemo-radiation therapy). Nine of these patients (40.9\%) received additional cycles of pre-operative systemic chemotherapy (i.e. not as chemo-sensitizer). Furthermore, one patient (4.5\%) did not receive radiation therapy of the primary tumour, but this patient did receive pre-operative chemotherapy. All of the patients who received pre-operative chemotherapy received an oxaliplatin-based chemotherapy regimen. Moreover, the majority of patients $(n=10)$ received one or more cycles including biologicals (i.e. bevacizumab). Four patients (18.2\%) received interval chemotherapy in the surgical window between hepatic and colorectal surgery, excluding those patients who did not undergo resection of their primary. Nine patients (40.9\%) who completed the curative intent treatment plan received adjuvant chemotherapy after completion of both operations. For those patients who received chemotherapy after their liver resection (either interval of adjuvant), this generally also consisted of an oxaliplatin-based regimen.

\section{DETAILS OF SURGERY}

Of the 22 patients in whom a liver-first approach was planned, in one patient (4.5\%) no liver resection was performed owing to a per-operative diagnosis of locoregional perihilar lymph nodal disease. These lymph nodes were located adjacent to the vena cava, making perfor- 
mance of a lymphadenectomy impossible. The operative characteristics of the 21 patients who did undergo a liver resection are detailed in Table 2.

\begin{tabular}{|c|c|}
\hline Variable & No. of patients $(\%), n=22$ \\
\hline \multicolumn{2}{|l|}{ Type of liver resection } \\
\hline None & $1(4.5)$ \\
\hline \multicolumn{2}{|l|}{ Single stage resection } \\
\hline$<$ Hemihepatectomy & $11(50.0)$ \\
\hline Hemihepatectomy & $8(36.4)$ \\
\hline Plus additional minor resection & $4(18.2)$ \\
\hline Central hepatectomy & $1(4.5)$ \\
\hline \multicolumn{2}{|l|}{ Two-stage resection } \\
\hline $\begin{array}{l}\text { Triple metastasectomy and subsequent } \\
\text { hemihepatectomy }\end{array}$ & $1(4.5)$ \\
\hline \multicolumn{2}{|l|}{ Type of colorectal resection } \\
\hline None & $6(27.3)$ \\
\hline Watchful wait & $1(4.5)$ \\
\hline Extensive metastatic disease & $4(18.2)$ \\
\hline Will follow & $1(4.5)$ \\
\hline Transanal endoscopic microsurgery & $1(4.5)$ \\
\hline Low anterior resection & $9(40.9)$ \\
\hline With diverting stoma & $6(27.3)$ \\
\hline Hartmann's resection & $2(9.1)$ \\
\hline With revision stoma & $1(4.5)$ \\
\hline Right hemicolectomy & $1(4.5)$ \\
\hline Rectum extirpation with end stoma & $2(9.1)$ \\
\hline Rectosigmoid resection with end stoma & $1(4.5)$ \\
\hline Median of lymph nodes harvested [range] & 12 [4-14] \\
\hline
\end{tabular}

Importantly, one patient (4.5\%) underwent a planned-two stage approach for his bilateral CRLM. This patient also underwent portal vein ligation (PVL) of the right portal vein during the first stage of his treatment. After a median interval of 3 months [range: 1-11], 16 patients $(72.7 \%)$ underwent surgery for their primary tumor. The operative characteristics of these 16 patients are detailed in Table 2. Of the five patients who did not undergo surgery or their primary tumor, three patients (13.6\%) developed extensive metastatic disease and were therefore not thought to be candidates for resection. Specifically, in two of these latter patients, the extrahepatic metastasis were diagnosed during attempted colorectal resection. Furthermore, in two patients $(9.1 \%)$ the primary tumor had disappeared due to the chemo-radiation therapy and therefore in these patients a wait-and-see approach was conducted, in accordance with a watchful wait protocol currently executed at our institu- 
tion. A complete response of the primary tumor was diagnosed by endoscopic examination and was pathologically confirmed by a biopsy of the region of the lesion. Altogether, the total number of patients not treated according to protocol was four (18.2\%), whereby excluding the two patients who are presently included in the follow-up protocol for complete responders to chemo-radiation. (Figure 1) Upon comparing patients who completed their per-protocol treatment and patients who deviated from the protocol, no differences were observed with regard to location of the primary tumor, size and number of CRLM or the distribution of the lesions (all $\mathrm{p}>0.05$ ). Moreover, there was no statistically significant difference in likelihood of succeeding between patients who had a symptomatic primary lesion and patients who did not $(p=0.63)$.

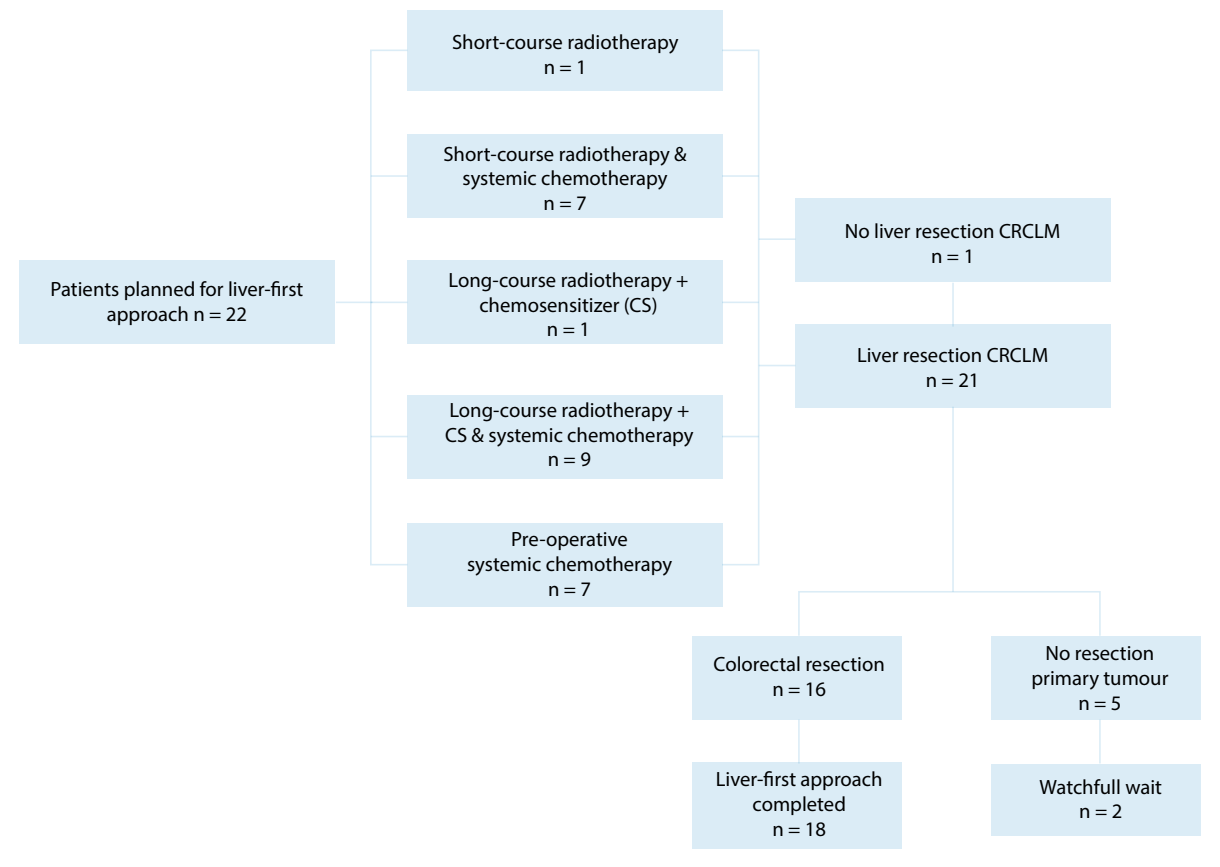

Figure 1 Flow chart of the 22 patients included in the present study, CRLM, colorectal liver metastasis:

\section{PATHOLOGIC CHARACTERISTICS}

The median number of CRLM resected was 2 [range: 1-7], with a median size of the largest lesion of $1.7 \mathrm{~cm}$ [range: $0.4-6.6$ ]. On final pathological analysis, 20 patients (95.2\%) had negative hepatic margins (R0)[25]. Moreover, in one patient (4.8\%), no viable tumor was found. Excluding patients who did not undergo resection of their primary colorectal tumor and therefore did not have pathologic staging of their primary tumor, most patients $(n=14$; $87.5 \%$ ) had a ypT3 (i.e. invasion through the muscularis propria into pericolorectal tissues) or a ypT4 (i.e. direct invasion in adjacent organs or structures) primary tumor[26]. Six patients 
(37.5\%) were found to have positive lymph nodes, therefore 10 patients $(62.5 \%)$ had node negative disease however all in the setting of pre-operative chemotherapy (ypN0).

The complication rate during hospital admittance following liver-directed surgery, or the 90-day post-operative period, was $27.3 \%(n=6)$, all of which were major complications (Clavien Grade 23 )[27]. The specifics of post-operative morbidity are detailed in Table 3. Importantly, there was no significant difference in the interval between hepatic surgery and the resection of the primary tumor when patients who did develop complications (median: 3.4 months [range: 3.3-9.8]) were compared with patients who did not (median: 2.3 months [range: 1.0-10.9]) $(p=0.095)$. Moreover, eight patients $(44.4 \%)$ developed post-operative complications after (attempted) resection of their primary tumor $(n=18)$, of whom half $(n=4$; 22.2\%) had also previously developed post-operative morbidity following liver-directed surgery. While two patients (11.1\%) developed minor complications (Clavien Grade <3), six patients (33.3\%) developed major complications (Clavien Grade $\geq 3$ )[27]. Importantly, one patient (5.4\%) died within 90 days of operation for the primary tumor, on post-operative day 74. At time of last follow-up, a-third $(n=6 ; 33.3 \%)$ of patients who underwent the complete curative paradigm or in whom a wait-and-see approach was conducted had developed recurrent disease. Specifically, none of the two patients in whom a wait-and-see approach was conducted experienced a local recurrence. The median recurrence-free survival following hepatic surgery was 14.5 months. The pattern of recurrence was combined intra- and extrahepatic recurrence in four patients $(25.0 \%)$, while another two patients $(12.5 \%)$ developed recurrent disease outside of the liver only. No patient developed recurrent disease solely in the liver. On an intention-to-treat basis, the estimated overall 1and

3-year survival calculated from the time of liver surgery were $74.2 \%$ and $41.1 \%$, respectively, with a median survival of 35.5 months. (Figure 2)

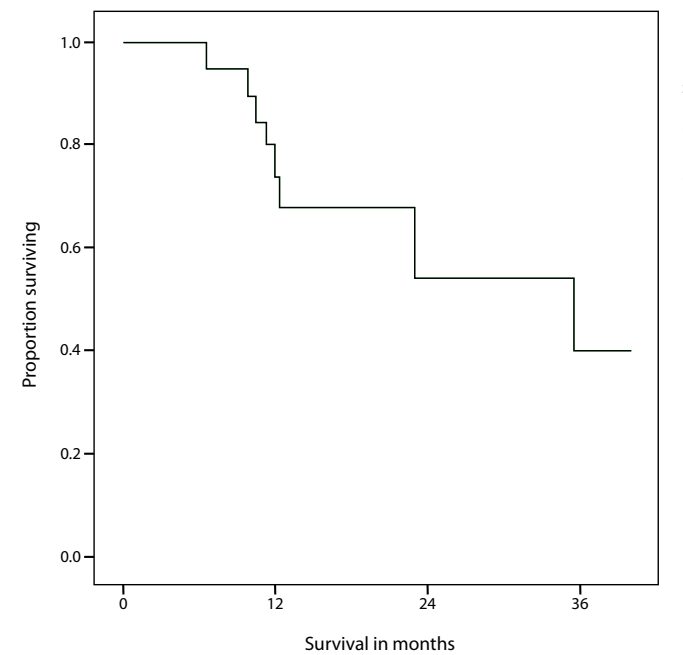

Figure 2 Kaplan-Meier curve showing overall
survival of the 22 patients who were planned to
undergo the liver-first approach
(intention-to-treat analysis)

\begin{tabular}{|l|llll|}
\hline Number of events & 0 & 5 & 7 & 8 \\
\hline Number at risk & 22 & 12 & 4 & 3 \\
\hline
\end{tabular}




\begin{tabular}{|c|c|}
\hline Variable & No. of patients (\%), $n=22$ \\
\hline \multicolumn{2}{|l|}{ Liver-directed surgery } \\
\hline Post-operative complications (any) & $6(27.3)$ \\
\hline Minor (Clavien Grade <3) & 0 \\
\hline Major (Clavien Grade $\geq 3$ ) & $6(27.3)$ \\
\hline Post-operative mortality (within 90 days) & 0 \\
\hline \multicolumn{2}{|l|}{ Specific complications } \\
\hline Biloma & $3(13.6)$ \\
\hline Intra-abdominal abscess & $1(4.5)$ \\
\hline latrogenic bowel perforation & $1(4.5)$ \\
\hline Stroke & $1(4.5)$ \\
\hline \multicolumn{2}{|l|}{ Surgery on primary colorectal tumor* } \\
\hline Post-operative complications (any) & $8(44.4)$ \\
\hline Minor (Clavien Grade <3) & $2(11.1)$ \\
\hline Major (Clavien Grade $\geq 3$ ) & $6(33.3)$ \\
\hline Post-operative mortality (within 90 days) & $1(5.6)$ \\
\hline \multicolumn{2}{|l|}{ Specific complications } \\
\hline Intra-abdominal abscess & $4(22.2)$ \\
\hline Anastomotic leakage & $1(5.6)$ \\
\hline Re-bleeding & $1(5.6)$ \\
\hline Pneumonia & $2(11.1)$ \\
\hline Fascia dehiscence & $1(5.6)$ \\
\hline Urinary tract infection & $1(5.6)$ \\
\hline Surgical site infection & $1(5.6)$ \\
\hline
\end{tabular}

Table 3 Specifics of direct post-operative outcome

\section{DISCUSSION}

Based on serious potential pitfalls of the traditional approach for synchronous CRLM, it has been proposed to reverse the order of the staged approach for these patients i.e. the liver-first approach. However, most data on this sequential in the treatment of patients with CRLM comes from a small series. Mortenson et al. described 26 patients in whom the CRLM were resected before the primary tumour[28]. However, in only a small portion of these patients, this therapeutic sequence was a planned strategy. Mentha et al. have reported on their experience on several occasions with the largest series including 35 patients, whereas Verhoef et al. reported on 23 patients in whom a liver-first approach was planned[12, 17, 18]. Moreover, this latter group reported on a cohort of 20 patients who all completed their liver-first strategy within a larger study on patients with synchronous CRLM[29]. Nonetheless, 
in this previous study, the specific characteristics of these 20 patients were not described in great detail. As previously mentioned, our institution has adopted the liver-first strategy several years ago, particularly in all patients who present with synchronous CRLM from a rectal origin but also in selected patients with synchronous CRLM from a primary tumour located in the colon, when an indication for pre-operative radiation therapy or chemotherapeutic regimens is present. In the current series, we report on 22 patients who were planned to undergo a liver-first approach during the 5 years of inclusion. Specifically, 18 patients $(81.8 \%)$ were treated per protocol and completed the treatment plan for liver and primary tumours. This percentage of feasibility is higher than the rate of patients who completed the formalized treatment plan reported by Verhoef et al. (69.6\%)[18]. Moreover, in the most recent series by Mentha et al. the authors report that $14 \%$ of patients did not complete the curative paradigm, while an additional $20 \%$ of patients had their primary tumour removed at the same time as the hepatic resection (i.e. a simultaneous approach)[12].

Therefore, in this previous study, merely $66 \%$ of patients were treated according to protocol. The feasibility of being able to complete the entire treatment protocol under the liver-first approach has been of some concern. As it has been stated that surgical management of CRLM is associated with an increased quality of life compared with chemotherapeutic therapy alone, an aggressive approach seems warranted[30]. In our cohort about one-fifth of patients were unable to complete their treatment paradigm, as four patients (18.2\%) deviated from the protocol as a result of the development of extrahepatic disease during the course of their treatment (i.e. under chemotherapy). It has been shown that tumour progression of CRLM under pre-operative chemotherapy is associated with a poor outcome, even after all (visible) metastatic disease is completely removed during curative intent surgery[15, 16]. This supports the potential benefit of the surgery-free interval as patients can be 'saved' an operation in the case of disease progression. In two patients (9.1\%), a complete response of the primary lesion owing to radiation therapy was observed. While the disappearance of the primary lesion has been shown to be of concern, with adequate follow-up and strict surveillance including regular evaluation of the CEA-level and appropriate cross-sectional imaging, patients can still be treated under a curative paradigm[31]. Therefore, these patients were not classified as treatment failures in the present study. Furthermore, although obstruction by the primary tumour during the course of treatment occurred in five patients (22.7\%), in all of these a diverting ostomy was constructed and in none of these patients this was a reason to divert from the planned strategy.

On another note, the potential surgical morbidity in managing patients with synchronous CRLM has also been an area of ongoing concern. Specifically, under the liver-first protocol, a complication after hepatic resection can result in a delay with regard to the treatment of the primary tumour. In our series, six patients (27.3\%) developed complications after liver-directed surgery, whereas the post-operative mortality was zero. These numbers cor- 
roborate the incidence of post-operative morbidity reported in other, larger series[32, 33]. Importantly, the interval between the operations for the hepatic and primary disease was similar for patients who did and patients who did not develop complications after liver surgery. Moreover, for patients in whom a too small future remnant is anticipated and who are therefore at an increased risk of developing hepatic failure, portal vein embolization (PVE) or PVL could be undertaken to enlarge the future liver remnant by means of hypertrophy, especially as patients treated with the liver-first approach are generally regarded to have advanced disease and undergo several cycles of pre-operative chemotherapy which could have subsequently caused hepatocyte damage[34,35]. After surgery on the primary tumour $(n=18)$, post-operative morbidity occurred in eight patients (44.4\%). Notably, one patient (5.6\%) died during the 90 -day post-operative period. These complication rates are similar to the post-operative morbidity rates reported in cohorts of patients who had not previously undergone a liver resection[36, 37].

Apart from the short-term results of the liver-first approach, it is important to also emphasize the influence on long-term outcomes (i.e. recurrence and survival) in these patients. In the current series, on an intention-to-treat basis, the estimated overall 1-and 3-year survival calculated from the time of liver surgery were $74.2 \%$ and $41.1 \%$, respectively, with a median survival of 35.5 months. These statistics are corroborated by other, albeit limited, series on patients operated for synchronous CRLM, treated using the traditional approach or the liver-first strategy[3, 12,38]. However, owing to advances in medical oncology (e.g. modern chemotherapy regimens) $[39,40]$ as well as surgical oncology (e.g. performance of repeat resections and improved peri[41]operative outcome), the overall survival for all patients who present with CRLM has improved dramatically[42]. Therefore, it has become increasingly important to assess disease recurrence in order to draw conclusions with regard to the curative potential of the proposed treatment paradigm. In the current cohort, six (33.3\%) of the patients who were treated per-protocol had developed recurrent disease at the time of the last follow-up. Available data on recurrence after the liver-first approach are scarce and range from $25.0 \%$ to $66.7 \%$ at the time of the last follow-up $[12,18]$. However, as recurrence is associated with time passed since surgery, it is preferred to investigate this variable in a timeto-event modus rather that comparing the crude probabilities. In the present cohort, we found a median recurrence-free interval of 14.5 months. While other studies on the liver-first approach did not report a median recurrence-free interval, this interval is in concordance with those reported in assorted cohorts of patients with CRLM[43, 44]. Moreover, although the recurrence rates reported in the series on the liver-first approach varied greatly, they do show that disease recurrence is not uncommon and therefore, especially in the proposed treatment sequence, there seems to be a role for a multidisciplinary approach.

The present study has several limitations associated with the retrospective nature of the study. This limited number of patients reflects the highly selected nature of the cohort of pa- 
tients with synchronous CRLM who were considered for this comparatively novel approach. As a result of the small sample size, the study has limited statistical power and therefore the statistical analyses are also limited.

\section{CONCLUSION}

The present study shows that resection of hepatic metastasis before resection of the primary colorectal tumour can be performed with acceptable peri-operative mortality and morbidity rates, similar to the traditional treatment paradigm for synchronous CRLM. Especially when the primary tumour requires a neo-adjuvant treatment strategy that provides a window for the liver-first approach, this sequence of therapy should be considered. Although over onefifth of patients were not able to complete the entire treatment plan, an important finding of this series is that the anticipated pitfalls of this approach (i.e. growth of CRLM beyond resectability and bowel obstruction by the primary tumour) were not found to alter the proposed treatment strategy. Moreover, the here presented data show that patients with synchronous CRLM treated with the liver-first strat-egy derive a considerable overall-survival benefit, although disease-recurrence rates remain relatively high. Especially in light hereof, the selection of patients for this novel strategy should be individualized and incorporated in a multidisciplinary approach to achieve the best outcomes. 


\section{REFERENCES}

1. Bengmark, S. and L. Hafstrom, The natural history of primary and secondary malignant tumors of the liver. II. The prognosis for patients with hepatic metastases from gastric carcinoma verified by laparotomy and postmortem examination. Digestion, 1969. 2(3): p. 179-86.

2. McMillan, D.C. and C.S. McArdle, Epidemiology of colorectal liver metastases. Surg Oncol, 2007. 16(1): p. 3-5.

3. Tsai, M.S., et al., Clinicopathological features and prognosis in resectable synchronous and metachronous colorectal liver metastasis. Ann Surg Oncol, 2007. 14(2): p. 786-94.

4. Pawlik, T.M. and M.A. Choti, Surgical therapy for colorectal metastases to the liver. J Gastrointest Surg, 2007. 11(8): p. 1057-77.

5. Pawlik, T.M., R.D. Schulick, and M.A. Choti, Expanding criteria for resectability of colorectal liver metastases. Oncologist, 2008. 13(1): p. 51-64.

6. Vauthey, J.N., et al., Chemotherapy regimen predicts steatohepatitis and an increase in 90-day mortality after surgery for hepatic colorectal metastases. J Clin Oncol, 2006. 24(13): p. 2065-72.

7. Pawlik, T.M., et al., Preoperative chemotherapy for colorectal liver metastases: impact on hepatic histology and postoperative outcome. J Gastrointest Surg, 2007. 11(7): p. 860-8.

8. van Vledder, M.G., et al., Disappearing colorectal liver metastases after chemotherapy: should we be concerned? J Gastrointest Surg, 2010. 14(11): p. 1691-700.

9. Lyass, S., et al., Combined colon and hepatic resection for synchronous colorectal liver metastases. J Surg Oncol, 2001. 78(1): p. 17-21.

10. Martin, R., et al., Simultaneous liver and colorectal resections are safe for synchronous colorectal liver metastasis. J Am Coll Surg, 2003. 197(2): p. 233-41; discussion 241-2.

11. Martin, R.C., C.R. Scoggins, and K.M. McMasters, Safety and efficacy of microwave ablation of hepatic tumors: a prospective review of a 5-year experience. Ann Surg Oncol, 2010. 17(1): p. 171-8.

12. Mentha, G., et al., 'Liver first' approach in the treatment of colorectal cancer with synchronous liver metastases. Dig Surg, 2008. 25(6): p. 430-5.

13. Mentha, G., et al., Treatment strategies for the management of advanced colorectal liver metastases detected synchronously with the primary tumour. Eur J Surg Oncol, 2007. 33 Suppl 2: p. S76-83.

14. Van Dessel, E., et al., Defining the optimal therapy sequence in synchronous resectable liver metastases from colorectal cancer: a decision analysis approach. Acta Chir Belg, 2009. 109(3): p. 317-20.

15. Adam, R., et al., Tumor progression while on chemotherapy: a contraindication to liver resection for multiple colorectal metastases? Ann Surg, 2004. 240(6): p. 105261; discussion 1061-4.

16. Blazer, D.G., 3rd, et al., Pathologic response to preoperative chemotherapy: a new outcome end point after resection of hepatic colorectal metastases. J Clin Oncol, 2008. 26(33): p. 5344-51.

17. Mentha, G., et al., Neoadjuvant chemotherapy and resection of advanced synchronous liver metastases before treatment of the colorectal primary. Br J Surg, 2006. 93(7): p. 872-8.

18. Verhoef, C., et al., The "liver-first approach" for patients with locally advanced rectal cancer and synchronous liver metastases. Dis Colon Rectum, 2009. 52(1): p. 23-30.

19. Dello, S.A., et al., Prospective volumetric assessment of the liver on a personal computer by nonradiologists prior to partial hepatectomy. World J Surg, 2011. 35(2): p. 386-92.

20. Dello, S.A., et al., Liver volumetry plug and play: do it yourself with ImageJ. World J Surg, 2007. 31(11): p. 221521.

21. Clavien, P.A., et al., Protection of the liver during hepatic surgery. J Gastrointest Surg, 2004. 8(3): p. 313-27.

22. Lambregts, D.M., et al., Accuracy of gadofosveset-enhanced MRI for nodal staging and restaging in rectal cancer. Ann Surg, 2011. 253(3): p. 539-45. 
23. Oncoline, Oncoline Cancer Clinical Practice Guidelines - Colon Cancer (2.0). 2011.

24. Kaplan E.L., M.P., Nonparametric estimation from incomplete observations. J. Am Stat Assoc, 1958. 53: p. 457-481.

25. Wittekind, C., et al., A uniform residual tumor (R) classification: integration of the $\mathrm{R}$ classification and the circumferential margin status. Cancer, 2009. 115(15): p. 3483-8.

26. Edge S.B., B.D.R., Compton C.C., Fritz A.G., Greene F.L., Trotti A., AJCC Cancer Staging Manual, 7th edn. Chicago, IL: Springer, 2010.

27. Dindo, D., N. Demartines, and P.A. Clavien, Classification of surgical complications: a new proposal with evaluation in a cohort of 6336 patients and results of a survey. Ann Surg, 2004. 240(2): p. 205-13.

28. Mortenson, M.M., Curley S.A., Vauthey J.N., Beaty K., Abdalla E.K, Resection of hepatic metastases before the colorectal primary: An alternate strategy for synchronous disease. Ann Surg Oncol, 2008. 15: p. 55-56.

29. van der Pool, A.E., et al., Optimizing the outcome of surgery in patients with rectal cancer and synchronous liver metastases. Br J Surg, 2010. 97(3): p. 383-90.

30. Wiering, B., et al., Long-term global quality of life in patients treated for colorectal liver metastases. Br J Surg, 2011. 98(4): p. 565-71; discussion 571-2.

31. Habr-Gama, A., et al., Complete clinical response after neoadjuvant chemoradiation therapy for distal rectal cancer: characterization of clinical and endoscopic findings for standardization. Dis Colon Rectum, 2010. 53(12): p. $1692-8$.

32. Jarnagin, W.R., et al., Improvement in perioperative outcome after hepatic resection: analysis of 1,803 consecutive cases over the past decade. Ann Surg, 2002. 236(4): p. 397-406; discussion 406-7.

33. Poon, R.T., et al., Improving perioperative outcome expands the role of hepatectomy in management of benign and malignant hepatobiliary diseases: analysis of 1222 consecutive patients from a prospective database. Ann Surg, 2004. 240(4): p. 698-708; discussion 708-10. 34. Abdalla, E.K., et al., Extended hepatectomy in pa- tients with hepatobiliary malignancies with and without preoperative portal vein embolization. Arch Surg, 2002. 137(6): p. 675-80; discussion 680-1.

35. Vauthey, J.N., et al., Standardized measurement of the future liver remnant prior to extended liver resection: methodology and clinical associations. Surgery, 2000. 127(5): p. 512-9.

36. Bretagnol, F., et al., Rectal cancer surgery with or without bowel preparation: The French GRECCAR III multicenter single-blinded randomized trial. Ann Surg, 2010. 252(5): p. 863-8.

37. Scabini, S., et al., Colon and rectal surgery for cancer without mechanical bowel preparation: one-center randomized prospective trial. World J Surg Oncol, 2010. 8: p. 35.

38. Fujita, S., T. Akasu, and Y. Moriya, Resection of synchronous liver metastases from colorectal cancer. Jpn J Clin Oncol, 2000. 30(1): p. 7-11.

39. Chibaudel, B., et al., Can chemotherapy be discontinued in unresectable metastatic colorectal cancer? The GERCOR OPTIMOX2 Study. J Clin Oncol, 2009. 27(34): p. 5727-33.

40. Dy, G.K., et al., Long-term survivors of metastatic colorectal cancer treated with systemic chemotherapy alone: a North Central Cancer Treatment Group review of 3811 patients, N0144. Clin Colorectal Cancer, 2009. 8(2): p. 88-93.

41. Asiyanbola, B., et al., Operative mortality after hepatic resection: are literature-based rates broadly applicable? J Gastrointest Surg, 2008. 12(5): p. 842-51.

42. de Jong, M.C., et al., Repeat curative intent liver surgery is safe and effective for recurrent colorectal liver metastasis: results from an international multi-institutional analysis. J Gastrointest Surg, 2009. 13(12): p. 2141-51.

43. de Jong, M.C., et al., Rates and patterns of recurrence following curative intent surgery for colorectal liver metastasis: an international multi-institutional analysis of 1669 patients. Ann Surg, 2009. 250(3): p. 440-8.

44. D'Angelica, M., et al., Effect on outcome of recurrence patterns after hepatectomy for colorectal metastases. Ann Surg Oncol, 2011. 18(4): p. 1096-103. 

II. REDUCING THE RISKS OF LIVER SURGERY

LIVER VOLUMETRY PLUG AND PLAY: DO IT YOURSELF WITH IMAGEJ

S.A.W.G. Dello, R.M. van Dam J.J.G. Slangen, M.C.G. van de Poll, M.H.A. Bemelmans, J.W.M. Greve, R.G.H. Beets-Tan, S.J. Wigmore, C.H.C. Dejong.

World Journal of Surgery 2007, 31: 2215 - 2221

VIRTUAL LIVER RESECTION AND VOLUMETRIC ANALYSIS OF THE FUTURE LIVER REMNANT USING OPEN SOURCE IMAGE PROCESSING SOFTWARE J.R. van der Vorst, R.M. van Dam, R.S.A. van Stiphout, M.A. van den Broek, I.H. Hollander, A.G.H. Kessels, C.H.C. Dejong.

World Journal of Surgery 2010, 34:2426-2433

PROSPECTIVE VOLUMETRIC ASSESSMENT OF THE LIVER ON A PERSONAL COMPUTER BY NONRADIOLOGISTS PRIOR TO PARTIAL HEPATECTOMY S.A.W.G. Dello, J.H.M.B. Stoot, R.S.A. van Stiphout, J.G. Bloemen, S.J. Wigmore, C.H.C. Dejong, R.M. van Dam.

World Journal of Surgery 2011, 35: 386-392 


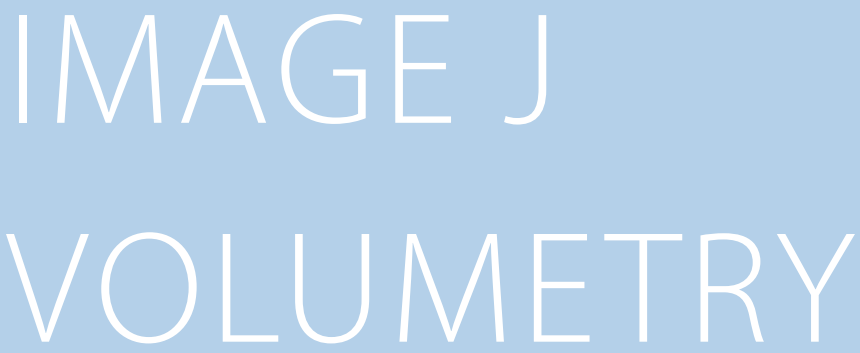

PUBLISHED AS

Liver Volumetry Plug and Play: Do It Yourself with ImageJ

S.A.W.G. Dello, R.M. van Dam, J.J.G. Slangen, M.C.G. van de Poll, M.H.A. Bemelmans, J.W.M. Greve, R.G.H. Beets-Tan, S.J. Wigmore, C.H.C. Dejong.

World Journal of Surgery 2007, 31: $2215-2221$

DOI: dx.doi.org/10.1007/s00268-007-9197-x

S.A.W.G. Dello and R.M. van Dam contributed equally to the realization of the study and the manuscript and share first authorship 


\section{ABSTRACT}

\section{BACKGROUND}

A small remnant liver volume is an important risk factor for posthepatectomy liver failure and can be predicted accurately by computed tomography (CT) volumetry using radiologic image analysis software. Unfortunately, this software is expensive and usually requires support by a radiologist. ImageJ is a freely downloadable image analysis software package developed by the National Institute of Health $(\mathrm{NIH})$ and brings liver volumetry to the surgeon's desktop. We aimed to assess the accuracy of ImageJ for hepatic CT volumetry.

\section{METHODS}

ImageJ was downloaded from http://www.rsb. info.nih.gov/ij/. Preoperative CT scans of 15 patients who underwent liver resection for colorectal cancer liver metastases were retrospectively analyzed. Scans were opened in ImageJ; and the liver, all metastases, and the intended parenchymal transection line were manually outlined on each slice. The area of each selected region, metastasis, resection specimen, and remnant liver was multiplied by the slice thickness to calculate volume. Volumes of virtual liver resection specimens measured with ImageJ were compared with specimen weights and calculated volumes obtained during pathology examination after resection.

\section{RESULTS}

There was an excellent correlation between the volumes calculated with ImageJ and the actual measured weights of the resection specimens $(r 2=0.98, p \backslash 0.0001)$. The weight/ volume ratio amounted to $0.88 \pm 0.04$ (standard error) and was in agreement with our earlier findings using CT-linked radiologic software.

\section{CONCLUSION}

ImageJ can be used for accurate hepatic CT volumetry on a personal computer. This application brings $\mathrm{CT}$ volumetry to the surgeon's desktop at no expense and is particularly useful in cases of tertiary referred patients, who already have a proper CT scan on CD-ROM from the referring institution. Most likely the discrepancy between volume and weight results from exsanguination of the liver after resection. 


\section{INTRODUCTION}

The incidence of colorectal cancer (CRC) is steadily increasing in the Western world [1, 2]. Approximately $50 \%$ of all patients diagnosed with CRC develop liver metastases at some stage of their disease. For these patients, liver resection is the only potentially curative treatment option. The number of candidates for liver resection is limited, however. One of the reasons is that liver dysfunction may occur when the extent of tumor involvement requires a major resection, leaving a small postoperative remnant liver volume[3-5]. As we and others have shown before, pre-and postoperative liver volumes can be accurately calculated from computed tomography (CT) or magnetic resonance imaging (MRI) scans[3, 6, 7]. Liver volumetry is useful for patient selection and helps reduce the incidence of complications due to insufficient residual liver volume[3, 5, 8, 9]. Unfortunately, radiologic image analysis software is typically linked to radiologic hardware, making it less accessible for non radiologists. In addition, the intended operation should be known to the investigator to predict the remnant liver volume accurately. This requires the expertise of a liver surgeon. Therefore, CT volumetry has hitherto been a multidisciplinary modality requiring the efforts of dedicated surgeons and radiologists. Advances in digitalization, the availability of broadband networks, and the introduction of CT scans on CD-ROM have enabled volumetry on a personal computer remote from radiological hardware (CT scanners or MRI). Advantages of stand-alone software are its applicability for tertiary referred patients, who already have a proper CT scan on CD-ROM from the referring institution and the possibility of performing liver volumetry independent of the input of a radiologist. However, commercially available stand-alone CT volumetry software is often expensive. Recently, an alternative approach using Adobe Photoshop was proposed to circumvent this problem, but the method is laborious[7]. ImageJ is a freely downloadable image analysis software package developed at the National Institute of Health (NIH) to assist in clinical and scientific image analyses. The applicability of ImageJ for liver volumetry has not been addressed before, but it potentially brings liver volumetry to the surgeon's desktop[10] . The objective of the present study was to establish the accuracy of ImageJ for CT volumetric analysis of the liver on a personal computer in patients undergoing major liver resection for CRC metastases.

\section{METHODS}

\section{PATIENTS, CT SCANS, LIVER WEIGHT}

In 2000, a prospective database was created in which data on all patients undergoing liver resection in our institution were collected. From this database, all patients who underwent major liver resection (2 3 segments) for colorectal metastases $(n=70)$ between 2000 and 2005 were selected. Subsequently, pathology files of these patients were checked by one of the authors (R.M.V.D.) to detect those cases in which liver specimens had been weighed by 
the pathologist. This yielded 15 patients (Table 1) who underwent major liver resection with a known specimen weight. Two of these patients underwent an extended procedure: one left hepatectomy + wedge resection and one right hepatectomy + segmentectomy.

Patients underwent CT scanning in their routine preoperative assessment either in our hospital or in one of the surrounding university-affiliated district general teaching hospitals. For volumetric analysis, four-phase CT scans were used that were provided on CD-ROM on four different viewers: eFilm Lite (eFilm Medical. Toronto, Canada), SIENET MagicView 300 VA42D (Siemens, Erlangen, Germany), DICOM CDViewer 3.412 (Quazar Software GmbH, Hamburg, Germany), or DICOM Lite-Box, version 2.02C (Rennes, France). The volumetric analyses were performed by two other investigators (S.A.W.G.D., J.J.G.S.) who were blinded to the weight of the resected specimens. Immediately following resection, the resection specimens were transferred to the pathology department by one of the surgeons to scrutinize resection margins. The specimens were weighed immediately upon arrival at that department.

\begin{tabular}{|c|c|}
\hline Variable & Results \\
\hline Age (years) & $60(34-77)$ \\
\hline $\operatorname{Sex}(M / F)$ & $7 / 8$ \\
\hline Height (cm) & $173(160-186)$ \\
\hline Body weight (kg) & $75(55-110)$ \\
\hline Body Mass Index & $24.8(21.5-38.1)$ \\
\hline Right hepatectomy (no.) & 12 \\
\hline Left hepatectomy (no.) & 3 \\
\hline Wedge-resection (VII) (no.) & 1 \\
\hline Segmentectomy (III) (no.) & 1 \\
\hline AST (IU/L) & $71(14-443)$ \\
\hline ALT (IU/L) & $69(14-392)$ \\
\hline LDH (IU/L) & $554(250-1617)$ \\
\hline Y-Glutamyltranspeptidase (IU/L) & $43(6-112)$ \\
\hline Alkaline phosphatase (IU/L) & $109(67-215)$ \\
\hline Bilirubin $(\mu \mathrm{M})$ & $18.4(9.2-47)$ \\
\hline
\end{tabular}

Table 1 Patient characteristics, results are the median and range or the number 


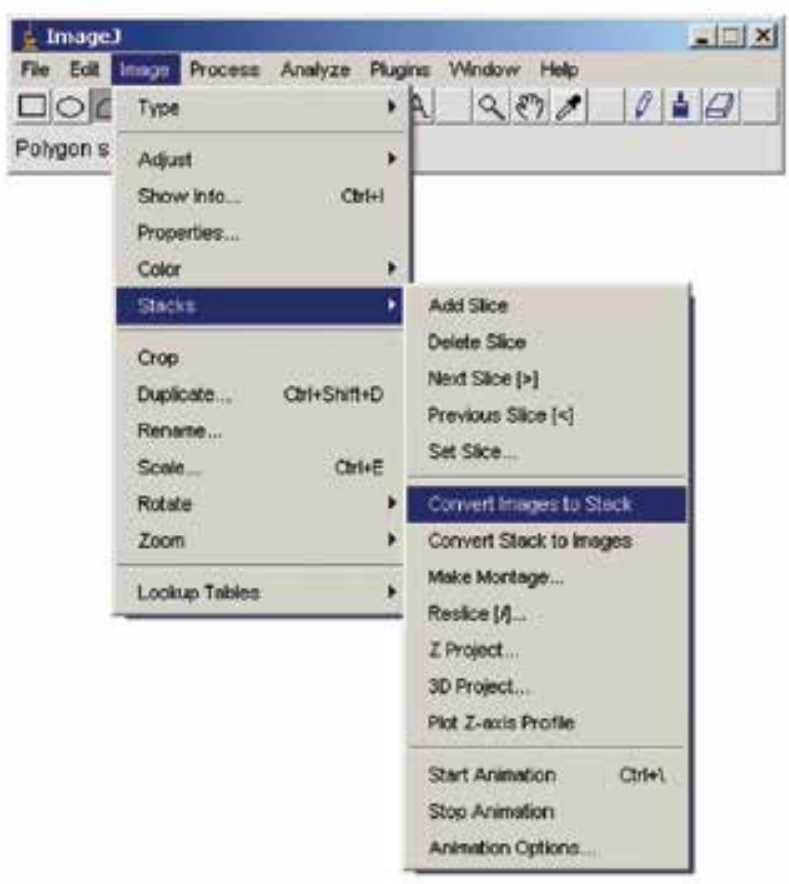

Fig. 1 ImageJ showing the "making a stack" pull-down menus

\section{CALCULATION OF LIVER VOLUME WITH IMAGE J}

Downloading ImageJ: ImageJ (version 1.33) was downloaded from http://www.rsb.info.nih. gov/ij/download.html (accession date: 01/07/2005).

\section{MAKING A STACK}

The portovenous phase of four-phase contrast-enhanced CT scans was used to facilitate optimal identification of liver segments and the anatomic resection plane in the individual patients. Relevant CT slices were evaluted in the original CT viewer. Every CT slice has a unique code or number that can be found in the information menu of the CT viewer, matching a JPEG file. The JPEG files were retrieved with Windows Explorer and opened in ImageJ by dragging them to the ImageJ main window. Individual slices were transformed to a "stack" using the function "Convert Images to Stack" in submenu "Stacks" in the pull-down menu "Image" (Fig. 1).

\section{ADJUSTING SCALE}

After opening DICOM images in ImageJ, the scaling of the images is corrected automatically, and volumetric analysis can be continued. However, in non-DICOM viewers, the scale of the imported stack was adjusted by measuring the distance between two randomly chosen but 
clearly recognizable points on a slice in the original CT viewer using its measurement tool. Subsequently, the line between these points was traced on the corresponding slice and its distance set in ImageJ using the "Set scale" function in the "Analyze" submenu.

\section{CREATING A REGION OF INTEREST}

On the CT slices there were three regions of interest (ROI) relevant for the present study: total liver area, metastasis area, and resection specimen area. Before outlining the ROI on each slice, the ROI manager in the pull-down menu "Analyze", submenu "Tools" was opened. The total liver, metastases, and resection specimen were manually outlined with the "Polygon selection tool."This tool can create an irregularly shaped selection defined by a series of line segments. To create an ROI on the CT slice, the mouse has to be clicked repeatedly to create line segments. When finished, one has to click in the small box at the starting point, and Image J automatically draws the last segment. The respective ROI of each slice was added to the $\mathrm{ROI}$ manager with the function "Add" in the ROI manager menu. The resection specimen was outlined according to transection planes as described in the operation notes. The gallbladder and the inferior caval vein were excluded from the ROI; intrahepatic biliary and vascular structures were included. In all patients with a hemihepatectomy, the transection line of the real liver resection followed Cantlies line from the top of the gallbladder, paralleling the middle hepatic vein straight to the suprahepatic inferior cava vein. In all cases the middle hepatic vein stayed in situ with the liver remnant (Fig. 2).

\section{CALCULATING VOLUME}

To calculate the areas, all the ROls must be selected in the ROI manager. The area of each $\mathrm{ROI}$ was calculated with the function "Measure" in the ROI manager menu. The calculated areas were then selected and copied (right click) to Microsoft Excel, where the areas were multiplied with slice thickness (ranging from 3 to $7 \mathrm{~mm}$, varying per viewer and/or patient). The last step was to add up these values per slice and calculate the volume of each three-dimensional structure. The data are the presented in cubic millimeters. Subsequently, these values per slice were added up to calculate the volume of each three-dimensional structure.

\section{TIME REQUIRED TO PERFORM LIVER CT-VOLUMETRY AND THE EFFECT OF SLICETHICKNESS}

To assess the time required to perform liver volumetry with ImageJ, the time needed to outline the three ROIs (total liver, metastases, resection specimen) per slice was recorded in one liver (a liver with one large tumor that required right hemihepatectomy). The time per slice was recorded, and the average time per slice for the entire liver was calculated. In addition, we calculated liver volumes using various slice thicknesses to evaluate the maximum slice thickness at which volumetry was stull accurate. 
STATISTICS

Results are presented as the median and range, as indicated in the Results section. The correlation between the actual weights of the resection specimens (determined during the pathology examination) and corresponding volumes calculated with ImageJ was evaluated with Pearson's test. A value of $p<0.05$ was considered to indicate statistical significance. Statistics were performed using Prism 4.0 for Windows (GraphPad Software, San Diego, CA, USA).

\section{RESULTS}

\section{PATIENTS}

Patient characteristics are shown in Table 1. The median age of the patients ( $n=15)$ was 60 years (range 34-77 years). None of the patients had any preoperative evidence of underlying liver disease, and routine liver tests were uniformly normal in all but one patient, who had slightly elevated plasma levels of liver enzymes. In total, 14 patients underwent a major anatomic resection. Right hepatectomy was performed in 11 patients, extended with a segmentectomy (segment III) in one. Left hepatectomy was performed in two patients, extended with a metastasectomy in segment VII in one. One patient underwent posterior sectionectomy extended with a segmentectomy of segment III, and one patient underwent left lateral sectionectomy. There were no postoperative deaths.

\section{RELATION BETWEEN RESECTED LIVER WEIGHT AND CT-MEASURED LIVER VOLUME}

The median resected liver volume calculated with ImageJ was $887 \mathrm{ml}$ (range 20-2173 ml). The median weight of the resection specimens measured during pathology examination was $732 \mathrm{~g}$ (range 23-1957 g). There was a highly significant correlation between resected liver volumes calculated with ImageJ and the actual measured weights of the resection specimens (Fig. 3)( $\mathrm{r} 2=0.98, \mathrm{p}<0.0001)$. The mean ratio between measured weight and calculated volume of the resection specimens was $0.88 \pm 0.04$ (SE) $\mathrm{g} / \mathrm{ml}$.

\section{CT-MEASURED TOTAL LIVER VOLUME/WEIGHT}

The calculated median total liver volume in our study population was $1610 \mathrm{ml}$ (range 1185$2990 \mathrm{ml}$ ), and the median total tumor volume was $63 \mathrm{ml}$ (range 4-1102 ml). The median functional liver volume (total liver volume - tumor volume = normal liver tissue volume) was $1606 \mathrm{ml}$ (range 944-2851 ml)

\section{TIME REQUIRED TO PERFORM CT VOLUMETRY AND EFFECT OF SLICETHICKNESS}

To assess the time required to perform volumetry with ImageJ, the time needed to outline the three relevant ROIs (total liver volume, metastases volume, and volume of the resection specimen) was recorded for one liver with a total volume of $1680.1 \mathrm{ml}$ and a metastases vol- 
ume of $32.8 \mathrm{ml}$ when right hemihepatectomy was performed. There were, in total, 34 slices; and analysis took on average of $50 \pm 3$ seconds per slice. Considering the average time needed per slice, it can be calculated that at a slice thickness of $5 \mathrm{~mm}$ a full volumetric analysis of a single liver requires on average of 25 to 28 minutes. Increasing the slice thickness results in a decline of accuracy of the volumetric assessment (Fig. 4). A maximum CT slice thickness of $1.0 \mathrm{~cm}$ reduces the number of slices needed to define an $\mathrm{ROI}$ and adds only a little error in the weight/volume ratio, thus providing an optimal balance between accuracy and time efficiency.

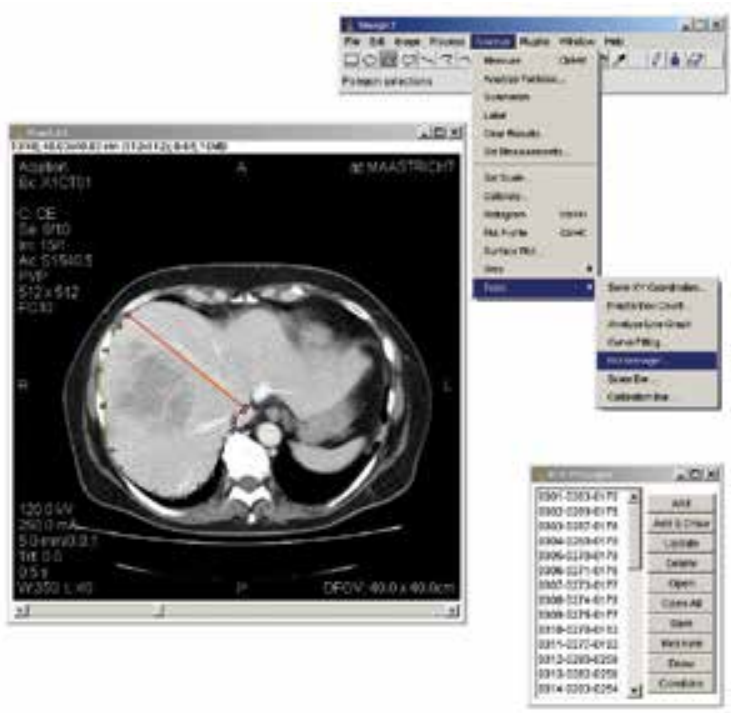

Fig. 2 Computed tomography (CT) scan opened in ImageJ showing the region of interest (ROI) pull-down menus and a large tumor in the right hemi-liver requiring right hepatectomy. The resection specimen is outlined on every slice as an ROI and added to the ROI manager (bottom right)

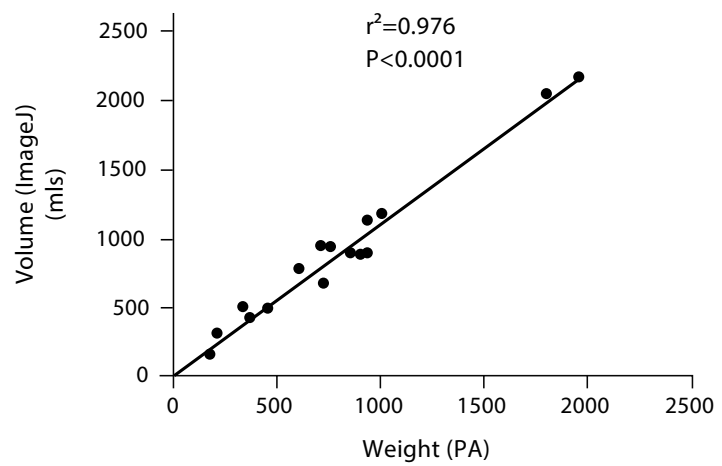

(g)

Fig. 3 Correlation between volume of resection calculated with ImageJ and actual measured weights of the resection specimens ( $n=15$, Pearson's test) 


\section{DISCUSSION}

In this study, we aimed to assess the accuracy of the freely downloadable image analysis software package ImageJ for hepatic CT volumetry in patients undergoing major hep-actectomy for colorectal cancer metastases. With the use of ImageJ, an accurate CT volumetric analysis can be performed on a personal computer, bringing liver volumetry within the surgeon's reach, independent of the support of a radiologist. The results are in agreement with our previous experience using professional radiological software[3,6], supporting the accuracy and reproducibility of CT volumetry with ImageJ.

In accordance with the study by Wigmore et al., we found a ratio between liver weight and CT-measured liver volume of $0.88 \pm 0.04$ (SE) $\mathrm{g} / \mathrm{ml}$ [6]. The most likely explanation of the systematic overestimation of liver volume compared to resected weight is that the three-dimensional reconstructions are based on a perfused liver, whereas the actual weight of the specimen was obtained directly after removal in a nonperfused state. Another explanation could be that the specific gravity of the liver is lower than $1 \mathrm{mg} / \mathrm{ml}$. However, the specific gravities of the various cellular and tissue components in the liver vary between $0.9 \mathrm{~g} / \mathrm{ml}$ (fat) and $1.10 \mathrm{mg} / \mathrm{ml}$ (hepatocytes)[11]. A specific gravity significantly $<1.0$ could only be explained by severe liver steatosis, which was found in only one of the resected specimens. Finally, aspiration of liver tissue by the Cavitron ultrasonic aspirator (CUSA) may contribute. At present, the limits of liver resection are being increasingly explored. Indications for liver resection are continuously being extended; and former contraindications, such as bilobar disease, number of metastases, and even extrahepatic disease, have been abandoned in selected cases[12-14].

In general, radical tumor clearance with a minimal resection margin of at least $1 \mathrm{~mm}$ is sufficient to achieve a significant survival benefit[15]. Consequently, postoperative remnant liver volume and function have become the most important determinants of resectability. Repeat liver resections, more extensive resections, and resections after aggressive induction chemotherapy are likely to yield more patients with small remnant livers and more with postresectional liver failure. The actual residual volume that limits resectability is still subject to debate, and the suggested minimal required remnant liver volume varies from $25 \%$ to $30 \%[3,4,16]$. In keeping with this, it has been shown that if meticulous attention is paid to the remnant liver volume and function, mortality can be decreased [5, 8, 9]. The variability in minimal required residual liver volume among individual patients is in all probability determined by the functional status of the parenchyma. It is common knowledge that major liver resection is less well tolerated in patients with cirrhosis or steatosis of the liver [17, 18]. Also, postchemotherapy nodular regenerative hyperplasia is considered not to have the same functional capacity as normal liver tissue. Consequently plain CT liver volumetry may not suffice to select patients for liver resection in these subgroups. True dynamic functional 
liver tests may become of additional use in these patients. In this context, it is worthwhile to mention some recent reports using a combination of $\mathrm{CT}$ volumetric analysis and functional tests such as indocyanine green (ICG) clearance tests or functional liver scintigraphy [19-21]. Equally, new methods become available to assess the role of the hepatic reticuloendothelial system and nitrogen clearance against the background of liver volumetry[22, 23]. The clinical applicability of such methods, however, awaits further confirmation.

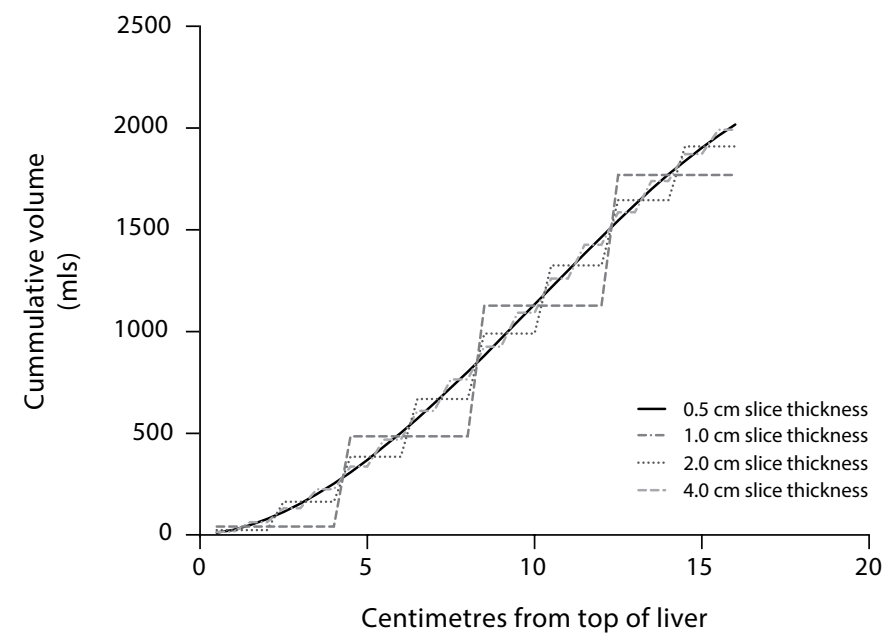

Fig. 4 Effect of increasing slice thickness on accuracy. It can be conceived clearly that a slice thickness of more than $2 \mathrm{~cm}$ leads to large deviations from the actual volume

A substantial number of patients admitted to specialized hepatobiliary units are referred from district general hospitals[24]. In our experience most of these patients already have a proper four-phase CT scan on CD-ROM from the referring institution. In most of these cases, however, it would not be possible to perform liver volumetry on these scans owing to incompatibility between radiologic software packages. ImageJ eliminates the necessity to perform an additional CT scan for the sole purpose of liver volumetry. Another advantage is that volumetry can be done by the surgeon without support from the radiology department. A potential disadvantage is the laboriousness of volumetry with ImageJ. Volumetric analyses takes on average $50 \pm 3$ seconds per slice. At a slice thickness of $5 \mathrm{~mm}$, a full volumetric analysis of a single liver requires on average 25 to 28 minutes for the entire liver.

The time required to perform the measurement can be limited by increasing slice thickness and thereby reducing the number of CT slices that have to be outlined. However, an increase in slice thickness is accompanied by decreased accuracy of the measurements. The results show that a maximum $C T$ slice thickness of $1.0 \mathrm{~cm}$ provides a good balance between accuracy and workload (Fig. 4). Time efficiency can be further improved by downloading 
a volume measurement "plug-in" that calculates the volume directly in the ROI manager. This eliminates the extra step of exporting calculated areas to Microsoft Excel. The plugin can be downloaded freely from https://list.nih.gov/cgi-bin/ wa?A2=ind0202\&L=IMAGE$J \& P=R 11200 \& l=-3$.

The accuracy of ImageJ to predict postoperative residual liver volume prospectively depends on the adherence to the intended resection line during the actual operation. In this retrospective study the transection plane could be carefully reconstructed based on the operation notes and guidance by the surgeons who performed the resection. The present study suggests that, in view of the excellent correlation between the predicted and actually resected weight, a reliable estimate of postoperative residual liver volume can be generated. All hepatectomies in the present study were anatomic resections. In the case of nonanatomical resection, it may be more difficult to predict the definitive transection plane and to outline it on a preoperative CT scan, but this problem would affect all software packages. Further studies are necessary to assess whether ImageJ provides the same volumetric accuracy for nonanatomic resections as for anatomic resections. In addition, evaluation of liver growth prior to repeated hepatectomy or following other volume-enhancing procedures, such as portal vein embolization, would be interesting potential fields of application for ImageJ[25].

\section{CONCLUSION}

ImageJ can be used for CT volumetric analysis of the liver on a personal computer. This application has the benefit that it is freely downloadable and brings CT volumetry to the surgeon's desktop independent of the support of a radiologist. ImageJ could be particularly useful in cases of tertiary referred patients who already have a proper CT scan on CD-ROM from the referring institution. 


\section{REFERENCES}

1. Jemal, A., et al., Cancer statistics, 2005. CA Cancer J Clin, 2005. 55(1): p. 10-30.

2. Visser, O., S. Siesling, and J.A. van Dijck, Incidence of cancer in The Netherlands 1999/2000]. Vereniging of Intergrale Kankercentra, Utrecht, 2003.

3. Schindl, M.J., et al., The value of residual liver volume as a predictor of hepatic dysfunction and infection after major liver resection. Gut, 2005. 54(2): p. 289-96.

4. Shoup, M., et al., Volumetric analysis predicts hepatic dysfunction in patients undergoing major liver resection. J Gastrointest Surg, 2003. 7(3): p. 325-30.

5. Jarnagin, W.R., et al., Improvement in perioperative outcome after hepatic resection: analysis of 1,803 consecutive cases over the past decade. Ann Surg, 2002. 236(4): p. 397-406; discussion 406-7.

6. Wigmore, S.J., et al., Virtual hepatic resection using three-dimensional reconstruction of helical computed tomography angioportograms. Ann Surg, 2001. 233(2): p. 221-6.

7. Lu, Y., et al., Hepatic volumetry with PhotoShop in personal computer. Hepatobiliary Pancreat Dis Int, 2004. 3(1): p. 82-5.

8. Dimick, J.B., et al., Hepatic resection in the United States: indications, outcomes, and hospital procedural volumes from a nationally representative database. Arch Surg, 2003. 138(2): p. 185-91.

9. Imamura, H., et al., One thousand fifty-six hepatectomies without mortality in 8 years. Arch Surg, 2003. 138(11): p. 1198-206; discussion 1206.

10. Rasband, W.S., ImageJ 1.33 edn. . U.S. National Institutes of Health, Bethesda, MD, 1997-2005.

11. Alpini, G., et al., Recent advances in the isolation of liver cells. Hepatology, 1994. 20(2): p. 494-514.

12. Shah, S.A., et al., Surgical resection of hepatic and pulmonary metastases from colorectal carcinoma. J Am Coll Surg, 2006. 202(3): p. 468-75.

13. Fusai, G. and B.R. Davidson, Management of colorectal liver metastases. Colorectal Dis, 2003. 5(1): p. 2-23.
14. Scheele, J., et al., [Resection of colorectal liver metastases. What prognostic factors determine patient selection?]. Chirurg, 2001. 72(5): p. 547-60.

15. Pawlik, T.M., et al., Effect of surgical margin status on survival and site of recurrence after hepatic resection for colorectal metastases. Ann Surg, 2005. 241(5): p. 715-22, discussion 722-4

16. Yigitler, C., et al., The small remnant liver after major liver resection: how common and how relevant? Liver Transpl, 2003. 9(9): p. S18-25.

17. Fan, S.T., Methods and related drawbacks in the estimation of surgical risks in cirrhotic patients undergoing hepatectomy. Hepatogastroenterology, 2002. 49(43): p. 17-20.

18. Behrns, K.E., et al., Hepatic steatosis as a potential risk factor for major hepatic resection. J Gastrointest Surg, 1998. 2(3): p. 292-8.

19. Fazakas, J., et al., Evaluation of liver function for hepatic resection. Transplant Proc, 2006. 38(3): p. 798-800. 20. Satoh, K., et al., 99mTc-GSA liver dynamic SPECT for the preoperative assessment of hepatectomy. Ann Nucl Med, 2003. 17(1): p. 61-7.

21. Bennink, R.J., et al., Preoperative assessment of postoperative remnant liver function using hepatobiliary scintigraphy. J Nucl Med, 2004. 45(6): p. 965-71.

22. Schindl, M.J., et al., The adaptive response of the reticuloendothelial system to major liver resection in humans. Ann Surg, 2006. 243(4): p. 507-14.

23. Dejong, C.H.C., Dowidar, N., Wigmore, S.J., Virtual hepatectomy and in vivo urea synthesis in patients with colorectal liver metastases. HPB (Oxford), 2001. 3(1): p. 114.

24. Majeed, A.W. and C. Price, Resource and manpower calculations for the provision of hepatobiliary surgical services in the UK. Ann R Coll Surg Engl, 2004. 86(2): p. 91-5.

25. Adam, R., et al., Two-stage hepatectomy: A planned strategy to treat irresectable liver tumors. Ann Surg, 2000. 232(6): p. 777-85. 



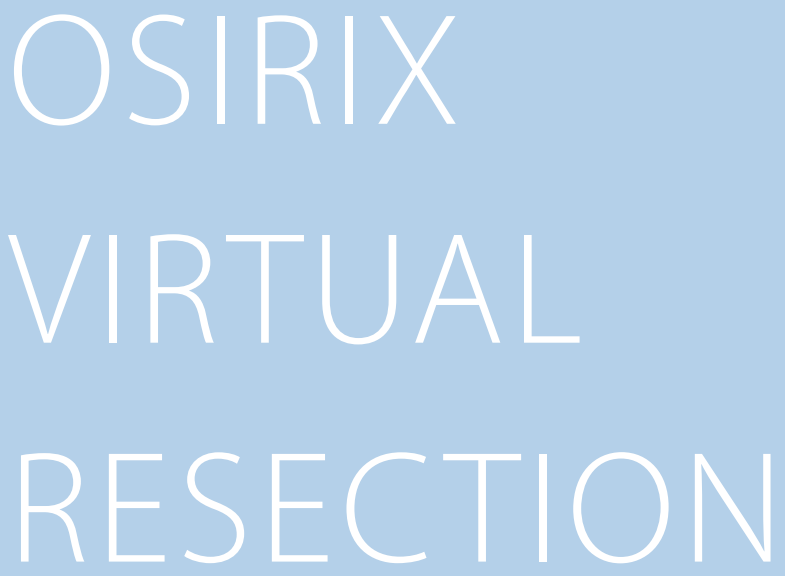

\section{PUBLISHED AS}

Virtual Liver Resection and Volumetric Analysis of the Future Liver Remnant using Open Source Image Processing Software

J.R. van der Vorst, R.M. van Dam, R.S.A. van Stiphout, M.A. van den Broek, I.H. Hollander, A.G.H. Kessels, C.H.C. Dejong.

World Journal of Surgery 2010, 34:2426-2433

DOI: dx.doi.org/10.1007/s00268-010-0663-5

J.R. van der Vorst and R.M. van Dam contributed equally to the realization of the study and the manuscript and share first authorship 


\section{ABSTRACT}

\section{BACKGROUND}

After extended liver resection, a remnant liver that is too small can lead to postresection liver failure. To reduce this risk, preoperative evaluation of the future liver remnant volume (FLRV) is critical. The open-source OsiriX PAC software system can be downloaded for free and used by nonradiologists to calculate liver volume using a stand-alone Apple computer. The purpose of this study was to assess the accuracy of OsiriX CT volumetry for predicting liver resection volume and FLVR in patients undergoing partial hepatectomy.

\section{METHODS}

Preoperative contrast-enhanced liver CT scans of patients who underwent partial hepatectomy were analyzed by three observers. Two surgical trainees measured the total liver volume, resection volume, and tumor volume using OsiriX, and a radiologist measured these volumes using CT scanner-linked Aquarius iNtuition software. Resection volume was correlated with prospectively determined resection weight, and differences in the measured liver volumes were analyzed. Interobserver variability was assessed using Bland-Altman plots.

\section{RESULTS}

25 patients (M/F ratio: 13/12) with a median age of 61 (range, 34-77) years were included. There were significant correlations between the weight and volume of the resected specimens (Pearson's correlation coefficient: $R^{2}=0.95$ ). There were no major differences in total liver volumes, resection volumes, or tumor volumes for observers 1, 2, and 3. Bland-Altman plots showed a small interobserver variability. The mean time to complete liver volumetry for one patient using OsiriX was $19 \pm 3 \mathrm{~min}$.

\section{CONCLUSION}

OsiriX liver volumetry performed by surgeons is an accurate and time-efficient method for predicting resection volume and FLRV. 


\section{INTRODUCTION}

Currently the focus of liver resection criteria is principally the limited functional reserve of liver tissue that remains after resection. Extended resection, staged resection, preoperative portal vein embolization to increase future remnant liver volume, and resection combined with tumor ablation is becoming standard in specialized liver units worldwide. More preoperative chemotherapy is being used in parallel with these aggressive strategies; as a consequence, more complicated and extended resections are being performed with reduced residual parenchymal function and smaller remnant livers [1].

Schindl et al. reported that partial liver resection can only be performed safely if the remnant liver volume is more than $26.6 \%$ of the total functional liver volume in patients with normal liver parenchyma [2]. The safe margin increases to $40 \%$ in patients with high-grade steatosis and after oxaliplatin-or irinotecan-based neoadjuvant chemotherapy, and the safe margins are $[50 \%$ of the total liver volume for cirrhotic livers $[2,3]$.

Both a small postoperative remnant liver size and an inappropriately functioning liver parenchyma increase the risk of postresectional liver failure (PLF) $[2,4,5]$. Preoperative assessment of future remnant liver volume and liver function is imperative to reduce this risk. Although accurate prediction of postresectional liver function remains difficult, there are a variety of tests used to assess this [6-10]. For example, the 15-minute indocyanine green retention test (ICG R15) measures the clearance capacity of the liver and is probably the most commonly used test in patients who undergo liver resection. To determine the maximum possible extent for safe liver resection, Makuuchi et al. and Clavien et al. proposed the use of decision trees that incorporate the ICG R15 [11, 12].

More recently, the 13C-methacetin LiMAx breath test has been proposed for assessing hepatic functional capacity [13].

A number of studies have validated tools to determine liver volume, with a particular focus on the volume of the future remnant liver. Computed tomography (CT) or magnetic resonance imaging (MRI) volumetry can be used to accurately predict total liver volume and future remnant liver volume [14-16]. Usually specialized hepatic radiologists perform the volumetric assessments using commercial software linked to radiological hardware. However, hepatic volumetry also can be performed accurately by a nonradiologist on a personal computer using open source ImageJ provided by the National Institutes of Health [[15]. Recently, a more user-friendly, freely downloadable open source image analysis software package, OsiriX, has become available. This software for Apple Mac OS has not yet been validated for liver CT volumetry. The purpose of this study was to assess the accuracy of OsiriXin performing liver CT volumetry by surgeons using personal computers and to compare the results to liver CT volumetry performed by a radiologist using classical radiological software (iNtuition) linked to a CT-scanner system. 


\section{METHODS}

\section{PATIENTS}

A database of patients undergoing liver resection in Maastricht University Medical Centre (Maastricht, The Netherlands) was created in 2000. Data of all patients were collected prospectively from 2000 onwards. From this database, all patients who underwent resection of two or more liver segments for primary or secondary liver tumors were selected if the resection specimen weight had been obtained in the operating room directly after resection $(n=110)$. From this group, the 25 most recently treated patients were selected and included for volumetric analysis. All resection specimens were postoperatively assessed by an experienced pathologist.

\section{CTVOLUMETRY}

Portal venous phase series of images from the preoperative contrast-enhanced CT scans were used for CT volumetry. Two observers ( $\mathrm{VV}$ and IH, both house officers; observers 1 and 2 , respectively) performed liver volumetry for all 25 patients on an iMac using OsiriX. Both observers were medical students in their final year of medical school who were trained in liver anatomy and the use of OsiriX by one of the liver surgeons (RD). A specialized liver radiologist (RS: observer 3 ) performed liver volumetry on exactly the same scans using iNtuition (TeraRecon, Houston, TX). iNtuition is the standard commercially available image analysis software package used in our liver unit. Total liver volume, resection volume, and tumor volume were determined using OsiriX (observers 1 and 2) or iNtuition (observer 3). Observers were blinded to the prospectively collected resection weights and to each other's results. On all slices, the gall bladder and the inferior caval vein were excluded in the regions of interest, and the intrahepatic vascular and biliary structures were included. For patients who underwent a hemihepatectomy, the transection line of the virtual liver resection followed Cantlie's line from the top of the gallbladder, paralleling the middle hepatic vein straight to the suprahepatic inferior caval vein. The middle hepatic vein was excluded from the virtual resection. Extended or extra-anatomic resections were guided by the operation notes. The total time required to perform liver volumetry was measured for all three observers.

\section{CALCULATION OF LIVER VOLUME WITH OSIRIX}

The 32-bit OsiriXversion 3.3 was downloaded from: http://www.osirix-viewer.com under the dropdown menu "downloads." A 2.8-Ghz Intel Core 2 Duo 24" iMac (Apple Inc., Cupertino, CA, USA) was used.

\section{LOADING A SERIES OF IMAGES INTO OSIRIX}

After inserting a CD-ROM or DVD containing CT scan data (including a commercial viewer), the "Digital Imaging and Communications in Medicine" (DICOM) data were automatically extracted from the disc by OsiriX and imported into the OsiriXviewer (Fig. 1a). 


\section{STORING DICOM DATA IN THE OSIRIX "PICTURE ARCHIVING AND COMMUNICATION SYSTEM" (PACS)}

DICOM data were stored in the OsiriX PACS using the "Copy linked files to Database folder" under "file" in the OsiriX dropdown menu.

\section{CONTOURING THE LIVER AND GENERATING REGIONS OF INTEREST}

Volumetry was performed on sets of axial images in the portal venous phase using a slice thickness of 3-5 mm. As a consequence of using CD-ROM containing DICOM CT scan data of referring centers, the slice thickness varied. Three kinds of volumetric assessments were performed prospectively in this study: total liver volume, resection volume, and tumor volume. The outline of the future resection specimen was traced manually on each slice with the "closed polygon selection" tool under the ROI tool button using a pen tablet system (Intuos 3, Wacom Co Itd., Saitama, Japan). The "Grow Region (2D/3D Segmenta-tion)" tool in the "ROI" dropdown menu made it possible to automatically outline the total liver and the metastases. This modality of OsiriX is based on the differences in Hounsfield units between the liver parenchyma, bony structures, and body fat. The automatic outlines are hand-adjusted with the "closed polygon selection" tool and "repulsor tool" to optimize the ROI (Fig. 1b).

\section{CALCULATING ACTUAL LIVER VOLUMES}

After selecting all of the regions of interest within one series, OsiriX automatically calculated the volume by multiplying surface and slice thickness and then adding up individual slice volumes. OsiriX also provided 3D images using the "ROI volume" tool (Fig. 1c).

\section{STATISTICAL ANALYSIS}

Actual resection weights obtained in the operating room were taken as the reference standard for CT volumetric resection measurements for both modalities. The volumes measured by the specialized radiologist using iNtuition were assumed to be the reference standard to validate OsiriX. Total liver volume, resection volume, and tumor volume were reported. The percentage of functional remnant liver volume (compared with the total functional volume) was calculated (FRLV\%). 


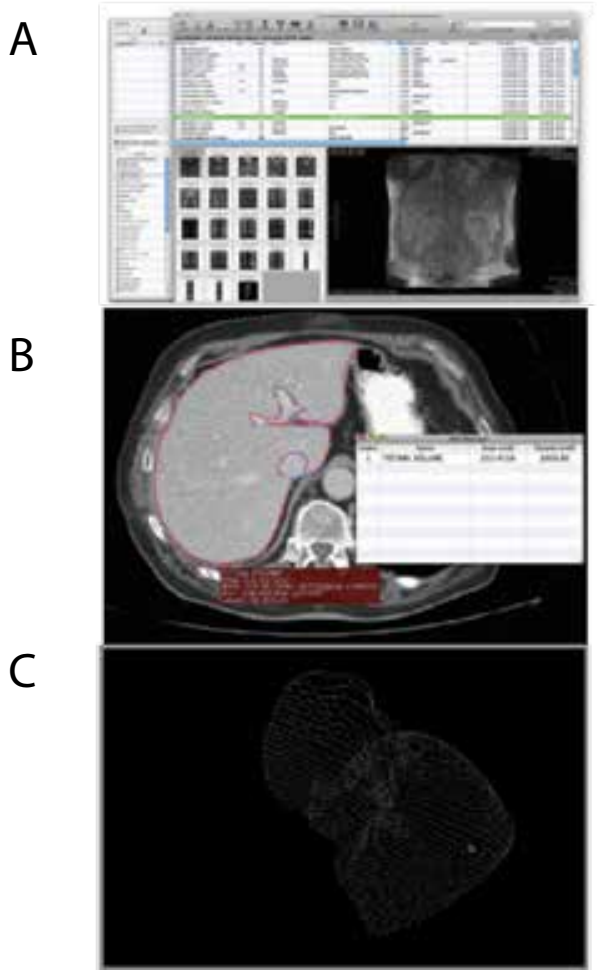

Fig.1 a Picture archiving and communication system integrated in Osirix Software package. b Outlining liver tissue. c 3D liver image

Unless otherwise stated, data are presented as the mean volume \pm standard deviation. Pearson's correlation coefficient $\mathrm{R}^{2}$ was used to test correlations between resection specimen weight and measured CT volumes using OsiriX. The mean differences between observers 1,2 , and 3 were tested with one-way ANOVA and paired $t$ tests corrected using the Bonferroni correction. Interobserver agreement was assessed with Bland-Altman plots. For total volume, resection volume, tumor volume, and FRLV\%, the average was plotted against the difference and $95 \%$ limits of agreement were provided. $P$ values $<0.05$ were considered significant. Statistical analysis was performed using GraphPad Prism software (version 5.01; California, USA).

Bland-Altman plots were used to assess intraobserver variability between OsiriX and iNtuition (observers 1 and 3) and between two observers using OsiriX (observers 1 and 2). For total volume, resection volume, tumor volume, and functional remnant liver volume percentages, the average was plotted against the difference and $95 \%$ limits of agreement were provided. 


\section{RESULTS}

\section{PATIENTS}

Twenty-five patients who underwent liver resection for colorectal cancer liver metastases were included in this study (Table 1). Five of 25 patients (20\%) were treated preoperatively with chemotherapy. Microscopic examination of the resection specimens revealed minor steatohepatitis in one of the patients. The liver parenchyma was normal in all others.

\section{SPECIMEN VOLUME AND RESECTION SPECIMEN WEIGHT}

For observers 1, 2, and 3, resection volumes assessed by OsiriX or iNtuition volumetry correlated strongly with actual resection weights obtained in the operating room immediately after resection $\left(\mathrm{R}^{2}=0.95,0.94\right.$, and 0.95 , respectively; Figure 2$)$. The resection weight-tovolume ratio measured by OsiriX was $0.85,0.86$, and $0.91 \mathrm{~g} / \mathrm{ml}$ for observers 1,2 , and 3 , respectively.

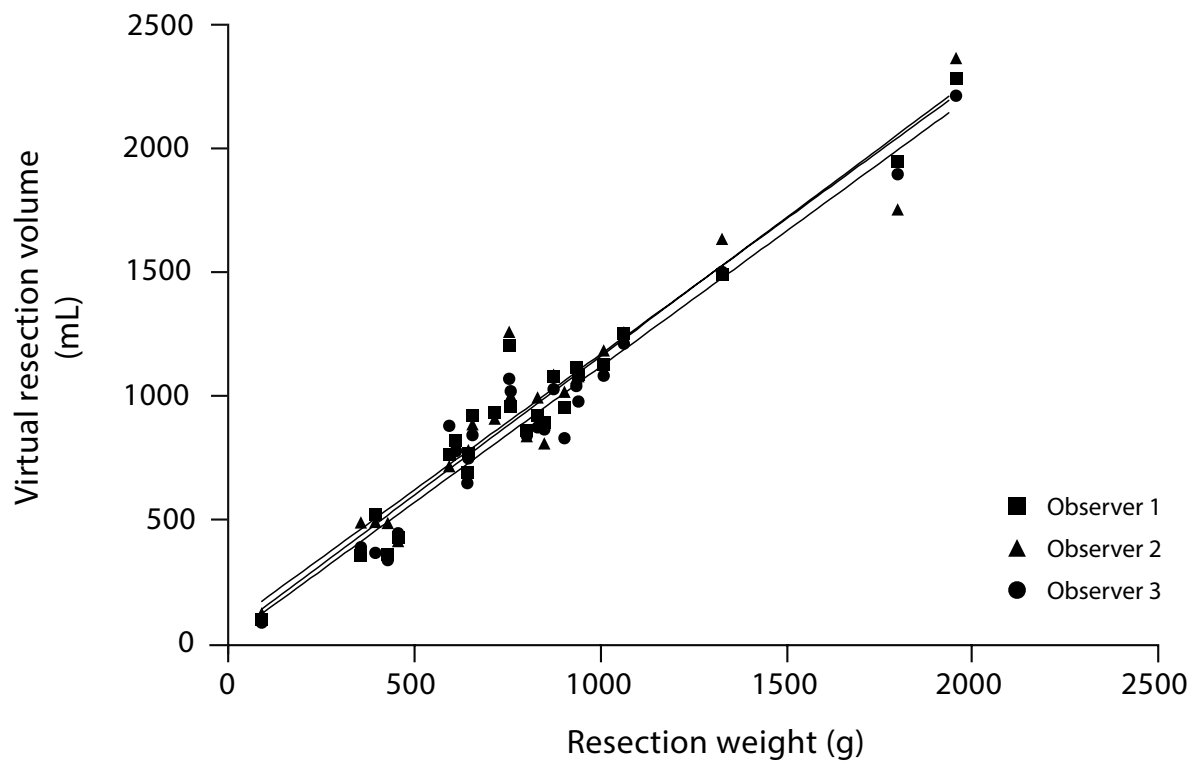

Fig. 2 Actual resection weight measured in the operating room directly after resection is plotted against the estimated resection volume by observers 1,2 , and 3 . 


\section{CT LIVER VOLUMES}

The FRLV\% was calculated, and all volumes were plotted for each observer (Figure 3). For the mean total liver volume, no significant differences were found between observer $1(1740 \mathrm{ml}$ $\pm 471.6)$, observer $2(1722 \mathrm{ml} \pm 466.3)$, and observer 3 (1683 $\mathrm{ml} \pm 460.8)$. For the mean resection volume, no significant differences were found between observer 1 ( $956 \mathrm{ml} \pm 462.7$ ), observer 2 (965 $\mathrm{ml} \pm 474.2)$, and observer $3(918 \mathrm{ml} \pm 461.9)$. There also were no significant differences found for the mean tumor volume measured by observer $1(84.58 \mathrm{ml} \pm 208.5)$, observer 2 (79.03 $\mathrm{ml} \pm 182.7$ ), and observer 3 ( $86.31 \mathrm{ml} \pm 215.3$ ). Finally, no significant differences were found in the mean FRLV\% for observer $1(46.27 \% \pm 17.86)$, observer $2(45.4 \% \pm$ $18.5)$, and observer $3(46.78 \% \pm 18.35)$.

\begin{tabular}{|l|l|}
\hline Characteristic & Results \\
\hline Mean age (y; range) & $61 ; 34-77$ \\
\hline Sex (M/F) & $13 / 12$ \\
\hline Right hepatectomy (no.) & 14 \\
\hline Right hepatectomy + metastasectomy (no.) & 5 \\
\hline Left hepatectomy (no.) & 1 \\
\hline Left hepatectomy + metastasectomy (no.) & 1 \\
\hline Extended right hepatectomy (no.) & 1 \\
\hline Left lateral hepatic sectionectomy (no.) & 3 \\
\hline
\end{tabular}

\section{INTRAOBSERVER VARIABILITY}

Liver volumetry measurements were compared using Bland-Altman plots, and the $95 \% \mathrm{lim}$ its of agreement were calculated (Figures. 4 and 5).

The mean time needed to perform complete volumetry in a patient (total liver volume, resection volume, and tumor volume) was $19 \pm 3 \mathrm{~min}$ when a 5 -mm scan slice thickness was used. 

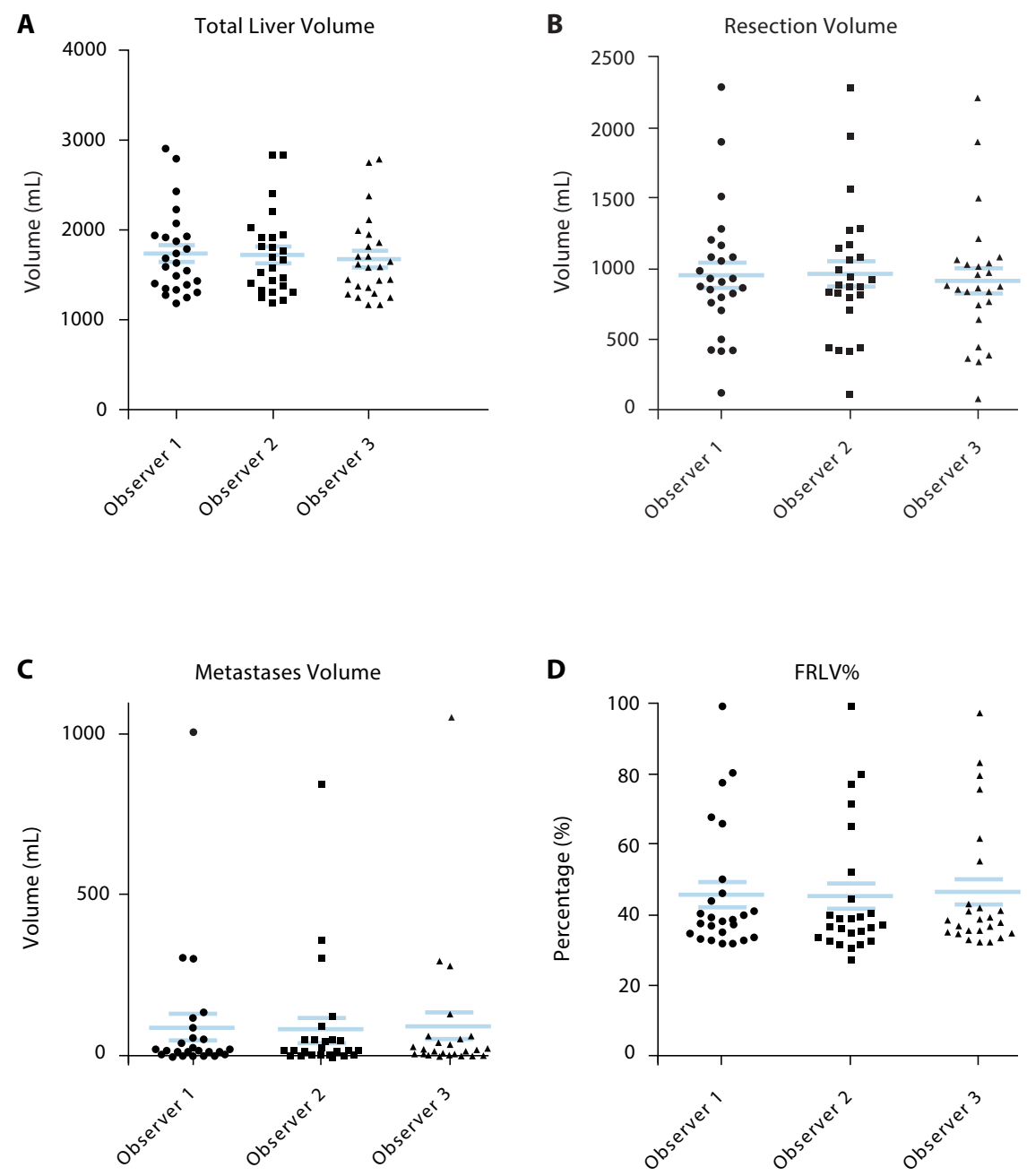

Fig.3 a .Mean total liver volume is plotted for observers 1, 2, and 3. Horizontal lines represent mean \pm SD. No significant differences were found. b. Mean resection volume is plotted for observers 1,2 , and 3 . Horizontal lines represent mean \pm SD. No significant differences were found. c. Mean metastases volume is plotted for observers 1,2 , and 3 . Horizontal lines represent mean \pm SD. No significant differences were found. d. Mean functional remnant liver volume as a percentage of total functional liver volume is plotted for observers 1,2 , and 3 . Horizontal lines 
A

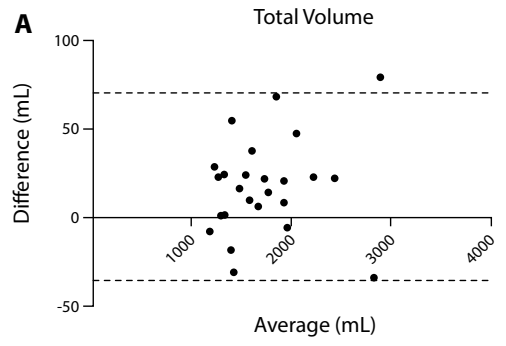

C

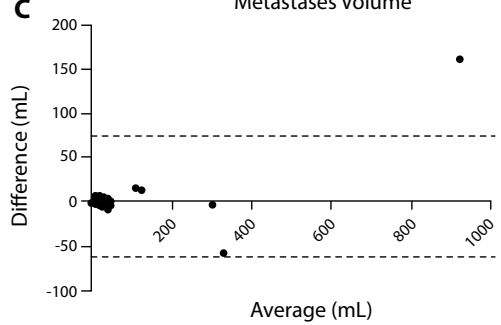

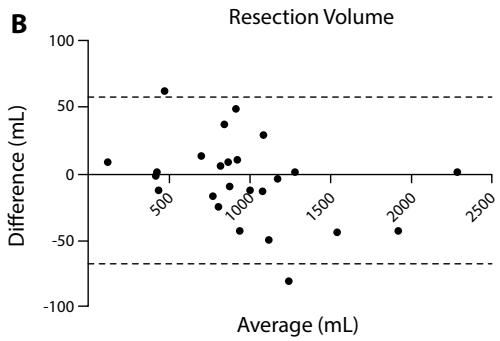

D

FLRV\%

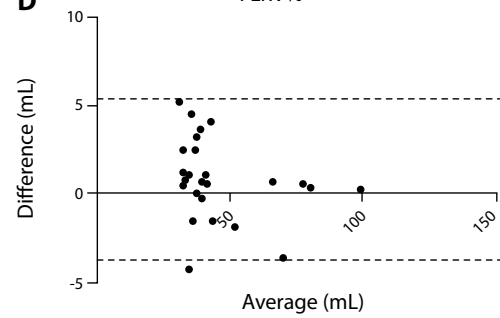

Fig. 4 a. Mean total volume of observers 1 and 2 is plotted against the difference in total volume between observers 1 and 2 . b. Mean resection volume of observers 1 and 2 is plotted against the difference in resection volume between observers 1 and 2 . c. Mean metastases volume of observers 1 and 2 is plotted against the difference in metastases volume between observers 1 and 2 . d. Mean functional remnant liver volume as a percentage of total functional liver volume of observers 1 and 2 is plotted against the difference in functional remnant liver volume as a percentage of total functional liver volume between observers 1 and 2. 
A
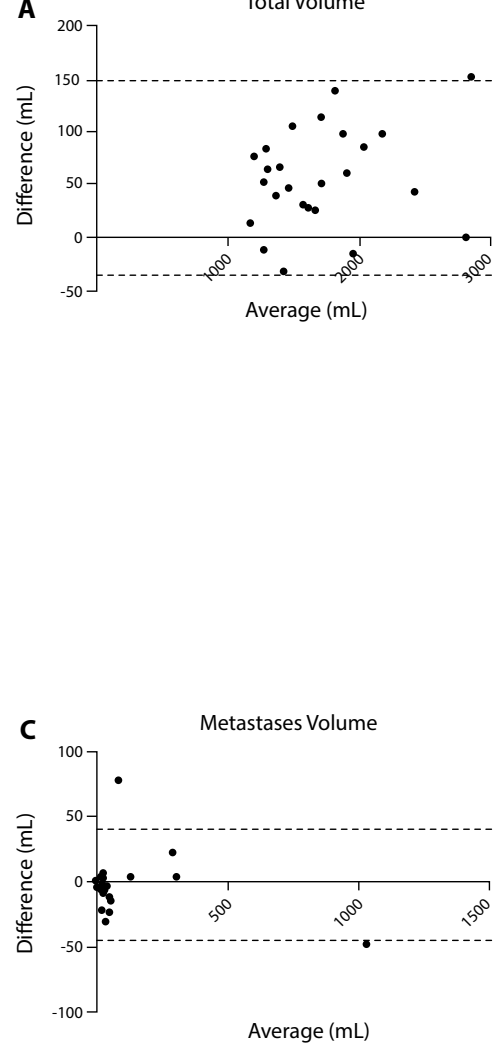
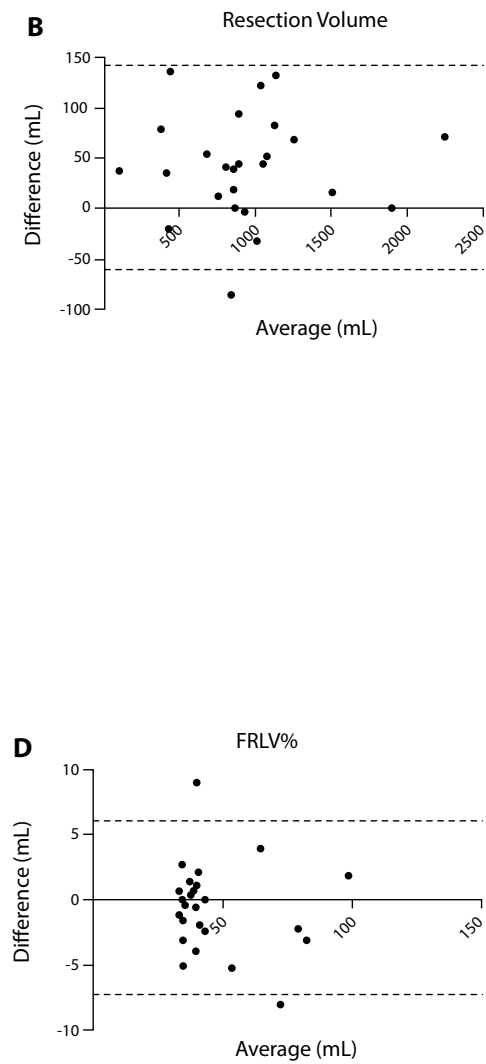

Fig.5 a. Mean total volume of observers 1 and 3 is plotted against the difference in total volume between observers 1 and 3 .

b. Mean resection volume of observers 1 and 3 is plotted against the difference in resection volume between observers 1 and 3 .

c. Mean metastases volume of observers 1 and 3 is plotted against the difference in metastases volume between observers 1 and 3 .

d. Mean functional remnant liver volume as a percentage of total functional liver volume of observers 1 and 3 is plotted against the difference in functional remnant liver volume as a percentage of total functional liver volume between observers 1 and 3. 


\section{DISCUSSION}

Accurate preoperative risk assessment to determine whether a patient can undergo major or extended liver resection remains the Holy Grail of liver surgery. In so far as volume equates to function, assessment of liver volume, and particularly the volume of the liver remnant, is critical. Unfortunately, liver volumetry is not always available, because it is usually linked to the CT scanner system and requires an experienced radiologist. User-friendly and easily accessible instruments are needed to predict remnant liver volume and parenchyma quality. OsiriX is an open source image analysis package and PAC system that can be downloaded free of charge. The goal of this study was to validate OsiriX for liver volumetry. The accuracy and efficiency of OsiriX for measuring liver volumes in patients undergoing partial liver resection was analyzed and compared with actual resection weights and CT liver volumetry using iNtuition software linked to the CT scanner system; the latter is currently the clinical standard at the Maastricht University Medical Centre.

In this study, we found a strong and highly significant correlation between resection weight and resection volume measured with OsiriX and iNtuition (Figure 2). The mean resection weight-to-volume ratio calculated with OsiriX was $0.85 \pm 0.11$ for observer $1,0.86 \pm 0.11 \mathrm{~g} /$ $\mathrm{ml}$ for observer 2 , and $0.91 \pm 0.13 \mathrm{~g} / \mathrm{ml}$ for observer 3 . These ratios and the systematic overestimation of the liver volume were in accordance with previous studies [14, 15]. A volumetric assessment of fully perfused livers compared with the weight of a resection specimen in a nonperfused state in the operating room could explain the systematic overestimation of liver volume. This, however, will not affect the functional remnant liver volume as a percentage of the total functional liver volume, which Schindl et al. reported to be an important determinant of the risk for developing postoperative liver failure [2].

To validate the OsiriX software package, volumetric assessments performed with OsiriX were compared with those performed with iNtuition. In addition, we compared the volumes measured by the two observers using OsiriX. One-way ANOVA and paired t tests showed that there were no significant differences in the mean volumes calculated with OsiriX and iNtuition or between the two observers using OsiriX. This means that trained surgical trainees using OsiriX for liver CT volumetry perform equally well as specialized radiologists using iNtuition. Although there were no significant differences in the mean volumes, the results of the two software packages at the individual patient level revealed some differences that must be noted. The differences in measured volumes at the individual patient level can be clarified using Bland-Altman plots (Figures. 4 and 5) and become particularly important in extended resections. In cases in which the preoperative liver volume assessment shows that the reported functional remnant liver volume percentage approaches $26.6 \%$, extra care is recommended. 
The measured volumes were consistently overestimated using iNtuition. In this study, we discovered that volumes calculated by iNtuition are inexplicably altered when the window level is changed, e.g., from abdominal to liver. This may have resulted in differences in volumetric results between the two packages as well as between subjects. Moreover, this casts some doubt as to whether iNtuition should be considered the "gold standard" for clinical use. Terra Recon (the company that makes iNtuition) is currently working on a solution to address this undesired phenomenon and is developing a dedicated liver tissue template. Future research will be needed to determine whether the new template is more accurate in assessing liver volume.

The mean time needed to analyze the total liver volume, tumor volume, and resection volume was $19 \pm 3 \mathrm{~min}$ at a slice thickness of $5 \mathrm{~mm}$. This is slightly faster than the time reported for ImageJ, which is another freely downloadable open source image analysis software package [15]. This can be explained by the semiautomatic selection of the resection specimen that is possible with OsiriX. Especially in the more caudal slices where there was a distinct difference in the Hounsfield units between liver parenchyma and the surrounding tissues, the semiautomatic contouring appeared to be very useful, fast, and precise. Additionally, it is possible to outline the liver on alternate individual slices. After outlining only half of the slices, the missing regions of interest can be generated using the "generate missing ROIs" tool in the ROI volume under the "ROI" dropdown menu. This method saves time because only small adjustments need to be made in the automatically generated regions of interest.

With OsiriX, surgeons can perform a virtual resection to predict the future functional remnant liver volume on their own computer. This enables optimization of preoperative planning and possibly reduces the risk of post resection liver failure, especially in extended resections. Furthermore, OsiriX has a big advantage in that it can be downloaded free of charge and it has an integrated PAC system in which the data of all examined patients are automatically stored. Nowadays, volumetric assessment of the liver is performed mainly by radiologists. At our center, the commercial image analysis software package iNtuition is used for liver CT volumetry. This package is not readily accessible to liver surgeons and hepatologists, which sometimes makes it difficult to use when considering different scenarios in the planning of extended resections. OsiriX combines an image archiving system and a user-friendly image analysis package. It allows one to store DICOM files of the CT scans of patients in a liver resection registry and is plug-and-play after downloading the software. However, the OsiriX system has not yet been validated for liver volumetry.

In the past we validated ImageJ, a software package developed by the National Institutes of Health, and found a high correlation between resection volume and actual resection weight. There are some drawbacks to ImageJ: patient DICOM files cannot be stored in a PAC system, it is not possible to semi automatically outline the liver, and missing regions of interest can- 
not be generated. Although the ImageJ system is accurate, it is our impression that it is less sophisticated than OsiriX. A potential obstacle for liver surgeons who want to use OsiriX is that it is only compatible with the Mac operating system. Although OsiriX can be used to perform liver volumetry, it does not accurately determine preoperative liver function because volume does not always equate to function. To predict the risk of developing post resection liver failure or infection, both function and volume are important. For patients treated with preoperative chemotherapy or suffering from liver cirrhosis, a larger remnant liver volume is needed to perform a safe resection. Because an accurate liver function test is not yet available, the safety of extended resections and resections in patients pretreated with chemotherapy remains uncertain. Nevertheless, CT volumetry of extended resections will offer additional information and reduce the risk of developing post resection liver failure.

\section{CONCLUSION}

The high correlation between resection weight and virtual resection volume, the weightto-volume ratios, and the acceptable low interobserver variability demonstrate that OsiriX can be used for CT volumetry of the liver. OsiriX accurately measures total liver volume, metastases volume, and virtual resection specimens. Using these measurements, clinicians are able to make an accurate prediction of the functional remnant liver volume. In addition, we found OsiriX to be a very well organized and efficient CT liver volumetry software package. 


\section{REFERENCES}

1. Khan, A.Z., G. Morris-Stiff, and M. Makuuchi, Patterns of chemotherapy-induced hepatic injury and their implications for patients undergoing liver resection for colorectal liver metastases. J Hepatobiliary Pancreat Surg, 2008.

2. Schindl, M.J., et al., The value of residual liver volume as a predictor of hepatic dysfunction and infection after major liver resection. Gut, 2005. 54(2): p. 289-96.

3. Ferrero, A., et al., Postoperative liver dysfunction and future remnant liver: where is the limit? Results of a prospective study. World J Surg, 2007. 31(8): p. 1643-51.

4. Jarnagin, W.R., et al., Improvement in perioperative outcome after hepatic resection: analysis of 1,803 consecutive cases over the past decade. Ann Surg, 2002. 236(4): p. 397-406; discussion 406-7.

5. Shoup, M., et al., Volumetric analysis predicts hepatic dysfunction in patients undergoing major liver resection. J Gastrointest Surg, 2003. 7(3): p. 325-30.

6. Armuzzi, A., et al., Review article: breath testing for human liver function assessment. Aliment Pharmacol Ther, 2002. 16(12): p. 1977-96.

7. Fazakas, J., et al., Evaluation of liver function for hepatic resection. Transplant Proc, 2006. 38(3): p. 798-800.

8. Gill, R.A., et al., Aminopyrine breath test predicts surgical risk for patients with liver disease. Ann Surg, 1983. 198(6): p. 701-4.

9. Schneider, P.D., Preoperative assessment of liver function. Surg Clin North Am, 2004. 84(2): p. 355-73.

10. Seyama, Y. and N. Kokudo, Assessment of liver function for safe hepatic resection. Hepatol Res, 2009. 39(2): p. 107-16.

11. Clavien, P.A., et al., Strategies for safer liver surgery and partial liver transplantation. N Engl J Med, 2007. 356(15): p. 1545-59.

12. Makuuchi, M., et al., Surgery for small liver cancers. Semin Surg Oncol, 1993. 9(4): p. 298-304.

13. Stockmann, M., et al., Prediction of postoperative outcome after hepatectomy with a new bedside test for maximal liver function capacity. Ann Surg, 2009. 250(1): p. 119-25.

14. Wigmore, S.J., et al., Virtual hepatic resection using three-dimensional reconstruction of helical computed tomography angioportograms. Ann Surg, 2001. 233(2): p. 221-6.

15. Dello, S.A., et al., Liver volumetry plug and play: do it yourself with ImageJ. World J Surg, 2007. 31(11): p. 221521.

16. Tu, R., et al., Assessment of hepatic functional reserve by cirrhosis grading and liver volume measurement using CT. World J Gastroenterol, 2007. 13(29): p. 3956-61. 

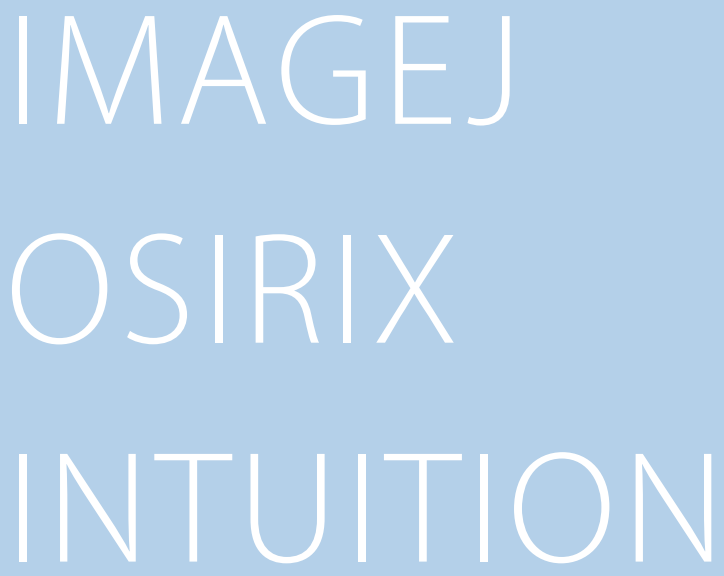

\section{PUBLISHED AS}

Prospective Volumetric Assessment of the Liver on a Personal Computer by Nonradiologists Prior to Partial Hepatectomy

S.A.W.G. Dello, J.H.M.B. Stoot, R.S.A. van Stiphout, J.G. Bloemen, S.J. Wigmore, C.H.C. Dejong, R.M. van Dam.

World Journal of Surgery 2011, 35: 386-392

DOI: dx.doi.org/10.1007/s00268-010-0877-6 


\section{ABSTRACT}

\section{BACKGROUND}

A small remnant liver volume is an important risk factor for posthepatectomy liver failure. ImageJ and OsiriX are both free, open-source image processing software packages. The aim of the present study was to compare ImageJ and OsiriX in performing prospective computed tomography (CT) volumetric analysis of the liver on a personal computer (PC) in patients undergoing major liver resection.

\section{METHODS}

Patients scheduled for a right hemihepatectomy were eligible for inclusion. Two surgeons and one surgical trainee measured volumes of total liver, tumor, and future resection specimen prospectively with ImageJ and OsiriX. A radiologist also measured these volumes with CT scanner-linked Aquarius iNtuition software. Resection volumes were compared with the actual weights of the liver specimens removed during surgery, and differences between the measured liver volumes were analyzed.

A total of 15 patients ( 8 men, 7 women) with a median age of 63 years (48-79 years) were included. There was a significant correlation between the measured weights of resection specimens and the volumes calculated prospectively with ImageJ and OsiriX $(r=0.89 ; r=$ 0.83 , respectively). There was also a significant correlation between the volumes measured with radiological software iNtuition and the volumes measured with ImageJ and OsiriX ( $r=$ $0.93 ; r=0.95$, respectively).

\section{CONCLUSION}

There were no major differences in total liver volumes, resection volumes, or tumour volumes for these three software packages. Prospective hepatic CT volumetry with ImageJ or OsiriX is reliable and can be accurately used on a PC by nonradiologists. ImageJ and OsiriX yield results comparable to the radiological software iNtuition. 


\section{INTRODUCTION}

Hepatectomies are performed increasingly more often, mainly because indications for liver resection are continuously being extended. Whereas in the past a number of exclusion criteria were applied to the selection of patients for hepatectomy, they have now been largely abandoned, and postoperative remnant liver volume and function have become the main determinants of resectability [1-3].

Postoperative liver dysfunction may occur when the extent of tumor involvement requires major liver resection (three or more segments), leaving a small postoperative remnant liver [4-6]. A reliable volumetric assessment of the part of the liver to be resected and the future residual liver volume should be a critical part of evaluation, particularly for extended resections, to prevent postoperative liver failure after resection. The safety of liver resection may increase if an estimate of minimal remnant liver volume is obtained via computed tomography $(\mathrm{CT})$ volumetry [4, 6-9]. This is especially important if the underlying liver parenchyma is abnormal or has impaired functional capacity. In this context, repeated liver resections, increasingly extensive resections, and resections after aggressive chemotherapy are likely to lead to more patients with small (functional) remnant livers and hence more risk of postoperative liver failure.

As we and others have shown before, liver volumes can be calculated accurately from CT or magnetic resonance imaging (MRI) scans [4, 10-12] [7, 13, 14]. The liberal use of existing professional image-processing software is often limited by costs, access, flexibility, lack of radiology staff, and specific hardware requirements. In addition, the intended operation and the potential surgical scenarios should be known to the investigator to predict the remnant liver volume accurately, which requires the expertise of a liver surgeon. The Digital Imaging and Communications in Medicine (DICOM) standard has enabled volumetry on a stand-alone or network-attached personal computer $(\mathrm{PC})$ remote from the radiology hardware (CT or MRI scanner). In 2007, our group conducted a retrospective study and proposed a method to perform liver volumetry with ImageJ, which is a free open-source image analysis software package developed at the National Institutes of Health (NIH) [8]. In 2010, our group assessed the accuracy of OsiriX for CT volumetry of the liver [11, 12]. OsiriX is a more user-friendly, freely downloadable open source image analysis software package for the Apple Mac OS. The accuracy of CT volumetry of the liver resection specimen prospectively prior to surgery has not yet been assessed for either of these software packages. Therefore, the objective of the present study was to compare ImageJ and OsiriX when performing prospective CT volumetric analysis of the liver on a PC in patients undergoing major liver resection. The study also planned to compare the prospective measurements of ImageJ and OsiriX to those of CT volumetry performed by a radiologist using classic radiology software (iNtuition) linked to a CT scanner system. 


\section{METHODS}

\section{PATIENTS}

Patients with primary or secondary liver tumors in otherwise normal livers (mostly colorectal cancer liver metastases) planned for a right hemihepatectomy at Maastricht University Medical Center between January 2008 and April 2009 were eligible for inclusion in this study. All patients had a contrast enhanced CT scan in their routine preoperative assessment either in our hospital or in one of the surrounding university-affiliated district general teaching hospitals. Patients were admitted to the hospital 1 day preoperatively, and routine blood tests were performed.

\section{SURGICAL PROCEDURE}

Liver resection was performed as detailed elsewhere [15]. In each patient, laparotomy was performed by bilateral subcostal incision, followed by intraoperative ultrasonographic (US) assessment of the liver. Once resectability had been confirmed, appropriate mobilization of the liver was performed as a prelude to hepatic parenchymal transection, which was undertaken using a Cavitron Ultrasonic Surgical Aspirator (Force GSU System; Valleylab, Boulder, CO, USA). Argon beam coagulation (Erbe, Tübingen, Germany), clips, and sutures were used for hemostasis. Immediately after liver resection, the weights of the resected specimens were recorded in the operating theater with a Mettler Toledo scale.

\section{CTVOLUMETRY}

For volumetric analysis, four-phase CT scans were used that were provided on CD-ROM on one of two viewers: eFilm Lite (eFilm Medical, Toronto, Canada) or SIENET MagicView 300 VA42D (Siemens, Erlangen, Germany).

\section{CALCULATION OF THE LIVER VOLUME USING IMAGEJ}

ImageJ (version 1.33) was downloaded from http://rsb.info.nih.gov/ij/download.html (accession date 1 January 2008). The portovenous phase of all contrast enhanced CT scans was used to facilitate optimal identification of liver segments and the anatomic resection plane. Details on how volumetry was performed with ImageJ have been described previously [11]. Briefly, the relevant source images were individually copied $(\mathrm{Ctrl}+\mathrm{V})$ from the CT viewer on the electronic patient care system and opened with ImageJ by dragging them to the ImageJ main window in the appropriate order. In cases of tertiary referred patients, relevant CT slices were searched in the original CT viewer on the CD-ROM. CT-slices on a CD-ROM were usually saved as compressed DICOM files, which cannot be opened or viewed via Windows Explorer. Therefore, the "Tudor DICOM Viewer Plug-in" was downloaded (free) from http:// imagejdocu.tudor.lu/ and installed in the ImageJ menu. This plug-in allowed us to preview the relevant $\mathrm{CT}$ files and open them directly in ImageJ.

The total liver, tumor, and resected specimen were manually outlined. The respective region 
of interest (ROI) of each slice was added to the ROI manager. The area of each ROI was calculated and then multiplied by the slice thickness. Finally, all slice volumes were added up to calculate the volume of each three-dimensional structure (i.e., total liver, resected specimen, tumor).

\section{PROSPECTIVE VOLUMETRIC ANALYSIS: IMAGEJ}

Volumetric analysis using ImageJ was performed prospectively by two nonradiologists (S.A.W.G.D., J.H.M.B.S.) during the preoperative evaluation of the patient and was blinded to the operating surgeon. For definition of the anatomic segments, Couinaud's classification was used. In all right hemihepatectomies, transection followed Cantlie's line from the top of the gallbladder, paralleling the middle hepatic vein, straight to the suprahepatic inferior caval vein. In all patients, the middle hepatic vein remained in situ with the liver remnant. Consequently, during the venous phase, the middle hepatic vein on CT was used to perform the volumetric measurement.

To assess the accuracy of the volumetric measurements of the liver with ImageJ, the volumes of the resected specimens were compared with the actual weights of the specimens. (The weights of the specimens had been recorded in the operating theater immediately after resection.) The actual weights of the resection specimens remained blinded to the investigators conducting CT volumetry until all patients had been included and analyzed prospectively. The functional remnant liver volume (FRLV) percentage was calculated with the following formula. FRLV [\%] $=100 \%$ x [total liver volume - resected volume] / [total liver volume - tumour liver volume]

\section{PROSPECTIVE VOLUMETRIC ANALYSIS: OSIRIX}

The 32-bit OsiriXversion 3.3 was downloaded from: http://www.osirix-viewer.com. A 2.8-GHz Intel Core 2 Duo 24 inch iMac (Apple, Cupertino, CA, USA) was used.

Details on how volumetry was performed with OsiriX have been described previously [12]. Volumes calculated with ImageJ were compared to the volumes obtained by CT volumetry using OsiriX by one of the liver surgeons (R.M.V.D.).

\section{COMPARISON OF IMAGEJ AND OSIRIXWITH INTUITION}

Volumes calculated with ImageJ and OsiriX were compared to the volumes obtained by CT volumetry performed by a specialized liver radiologist (R.S.A.van S.) using radiology image analysis software from TeraRecon linked to an Aquarius iNtuition workstation. Volumetric analysis performed with ImageJ and OsiriX was blinded to the radiologist. The volume of the liver resection specimen measured by the radiologist was used as a second standard of reference instead of the actual weight of the resected specimen. 


\section{RELIABILITY: IMAGEJ}

For analysis of interuser variability of ImageJ, volume measurements of total liver volume, functional liver volume, and tumor volume performed before surgery by two investigators (S.A.W.G.D., J.H.M.B.S.) were compared. The first investigator had 3 years' experience and the second investigator had no experience with the applied software and received instructions and training before starting with the measurements. Bland-Altman plots were used (see below, under Statistical Analysis) to analyze the reliability of the ImageJ method.

\section{TIME CONSUMPTION: IMAGEJ}

The time consumed to perform one volumetric assessment of a liver with ImageJ including the total liver, the tumor, and the resected specimen was recorded for each of the 15 livers. To assess time consumption, workload, and influence of slice thickness, the time needed to outline these three ROIs (total liver, tumor, resected specimen) was recorded per slice in five livers.

As for any volumetric measurement technique based on CT imaging data sets, the accuracy of the volume measurements is dependent on the slice thickness used for the volume calculation. The slice thickness used for volume measurement is also crucial for the time needed to perform the analysis because of manual outlining. Therefore, the influence of slice thickness on CT-based liver volume measurements using ImageJ was also investigated. Total liver volume (TLV) was analyzed based on different slice thicknesses in these patients undergoing a right hemihepatectomy. The relative change in TLV between volumetry performed with the standard of reference $(0.5 \mathrm{~cm})$ and volumetric measurements using thicker slices $(1.0,1.5$, and $2.0 \mathrm{~cm}$ ) was calculated with the following formulas. (TLV1.0 -TLV0.5)/TLV0.5 $\mathrm{x}$ 100\%; (TLV1.5 -TLV0.5) / TLV0.5 x 100\%; (TLV2.0 -TLV0.5) / TLV0.5 x 100\%; The relative gain of time between volumetric measurements using different slice thicknesses was recorded and calculated in percentages as well.

\section{STATISTICAL ANALYSIS}

Pearson's coefficient was used to quantify the association between the volume of the resected specimen and the actual weight of the resection specimen. A value of $P<0.05$ was considered to indicate statistical significance. The resulting regression line was described as a linear equation, and the correlation coefficient ( $r$ ) was calculated. Changes of calculated volumes were tested against zero with the use of a Wilcoxon signed rank test with a theoretical mean of zero. Data are presented as the mean \pm standard error of the mean (SEM). The mean and standard deviation (SD) were calculated from the two volumetric results for each user using ImageJ. Next, the limits of agreement as proposed by Bland and Altman were calculated [16]. This method is based on the mean and SD of the difference between two ratings of the same patient. The mean difference between two users indicates systematic error. The SD of the difference between two users indicates random error. The $95 \%$ limits of agreement as in Bland and Altman's plots result in both quantification of random error and 
systematic error (1.96 x SD) of the measurement method. Statistics were performed using Prism 5.0 for Windows (GraphPad Software, San Diego, CA, USA).

\section{RESULTS}

\section{PATIENTS}

Fifteen patients scheduled for right hemihepatectomy were included in the study. The median age of the patients was 63 years ( $48-79$ years). None of the patients had preoper-ative evidence of underlying liver disease, and routine liver function tests were uniformly normal (Table 1).

\begin{tabular}{|l|l|}
\hline Characteristic & Median (range) \\
\hline Age (years) & $63(48-79)$ \\
\hline Sex (M/F) & $8 / 7$ \\
\hline Height (cm) & $170(155-191)$ \\
\hline Weight (kg) & $76(55-127)$ \\
\hline BMI (kg/m2) & $26(19-43)$ \\
\hline AST (IU/I) & $22(7-52)$ \\
\hline ALT (IU/I) & $30(8-61)$ \\
\hline LDH (IU/I)) & $372(312-469$ \\
\hline yGGT (IU/I) & $37(29-258)$ \\
\hline ALP (IU/I) & $98(57-168)$ \\
\hline Bilirubin (IM) & $12(7-15)$ \\
\hline $\begin{array}{l}\text { BMI body mass index, AST aspartate aminotransferase, ALT alanine, ami- } \\
\text { notransferase, LDH lactate dehydrogenase, yGGT y-glutamyltranspeptidase, } \\
\text { ALP alkaline phosphatase }\end{array}$ & \\
\hline
\end{tabular}

Table 1 Patient characteristics

\section{RELATION BETWEEN PROSPECTIVE CT-MEASURED LIVER VOLUME \\ AND RESECTED LIVER WEIGHT}

The mean weight of the resection specimens measured in the operating theater was 788.8 $\pm 53.7 \mathrm{~g}$. The mean resected liver volumes calculated with ImageJ, OsiriX, and iNtuition were $987.7 \pm 64.0,944.7 \pm 64.1$, and $905.0 \pm 59.9 \mathrm{ml}$, respectively. The mean ratios between the measured weight and volume measured by ImageJ, OsiriX, and iNtuition were $0.78 \pm 0.03$, $0.81 \pm 0.04$, and $0.83 \pm 0.04$, respectively. The mean remnant liver volume percentages measured by ImageJ, OsiriX, and iNtuition were $41.4 \pm 1.6 \%, 36.2 \pm 1.5 \%$, and $37.8 \pm 1.6 \%$, respectively (Table 2).

In one patient two surgical procedures were possible, either a right posterior sectionectomy or a right hemihepatectomy. Both volumes were calculated prospectively, and finally the 
volume for right posterior sectionectomy was included in the analysis. There was a significant correlation between the resected liver volumes calculated with ImageJ, OsiriX, and iNtuition prospectively and the actual measured weights of the resection specimens: $r=0.89$ and $P<0.001 ; r=0.83$ and $P<0.001 ; r=0.85$ and $P<0.001$; respectively (Figure 1).

\begin{tabular}{|l|l|l|l|l|}
\hline Measurement & Image J User 1 & Image J User 2 & OsiriX & iNtuition \\
\hline Total liver volume $(\mathrm{ml})$ & $1736.0 \pm 104.0$ & $1785.0 \pm 114.9$ & $1536.0 \pm 98.6$ & $1540.0 \pm 99.9$ \\
\hline Resected liver volume $(\mathrm{ml})$ & $987.7 \pm 64.0$ & $1050.0 \pm 78.6$ & $944.7 \pm 64.1$ & $905.0 \pm 59.9$ \\
\hline Tumor volume $(\mathrm{ml})$ & $14.0 \pm 4.3$ & $18.9 \pm 4.4$ & $12.0 \pm 3.7$ & $12.4 \pm 4.0$ \\
\hline Remnant liver volume (\%) & $41.4 \pm 1.6$ & $39.0 \pm 1.4$ & $36.2 \pm 1.5$ & $37.8 \pm 1.6$ \\
\hline Resected specimen weight/volume ratio $(\mathrm{g} / \mathrm{ml})$ & $0.78 \pm 0.03$ & $0.77 \pm 0.03$ & $0.81 \pm 0.04$ & $0.83 \pm 0.04$ \\
\hline Resected specimen weight $(\mathrm{g})$ & $788.8 \pm 53.7$ & & & \\
\hline
\end{tabular}

Table 2 Results for 15 CT volumetry and intraoperative measurements

\section{INTEROBSERVER VARIABILITY: IMAGEJ}

Liver volumetry measurements using ImageJ were compared using Bland-Altman plots. The mean difference of TLV measurements between both users was $49.4 \pm 86.1 \mathrm{ml}$. The $95 \%$ limits of agreement were -218.2 to $119.4 \mathrm{ml}$ (Figure 2a). The mean difference of the liver resection specimen measurements between both users was $53.0 \mathrm{ml} \pm$

$89.8 \mathrm{ml}$. The $95 \%$ limits of agreement were -228.9 to $122.9 \mathrm{ml}$ (Figure $2 \mathrm{~b}$ ). The mean difference of the \%FRLV measurements between both users was $2.3 \pm 4.6 \%$. The $95 \%$ limits of agreement were $-6.7 \%$ to $11.4 \%$ (Fig. 2c).

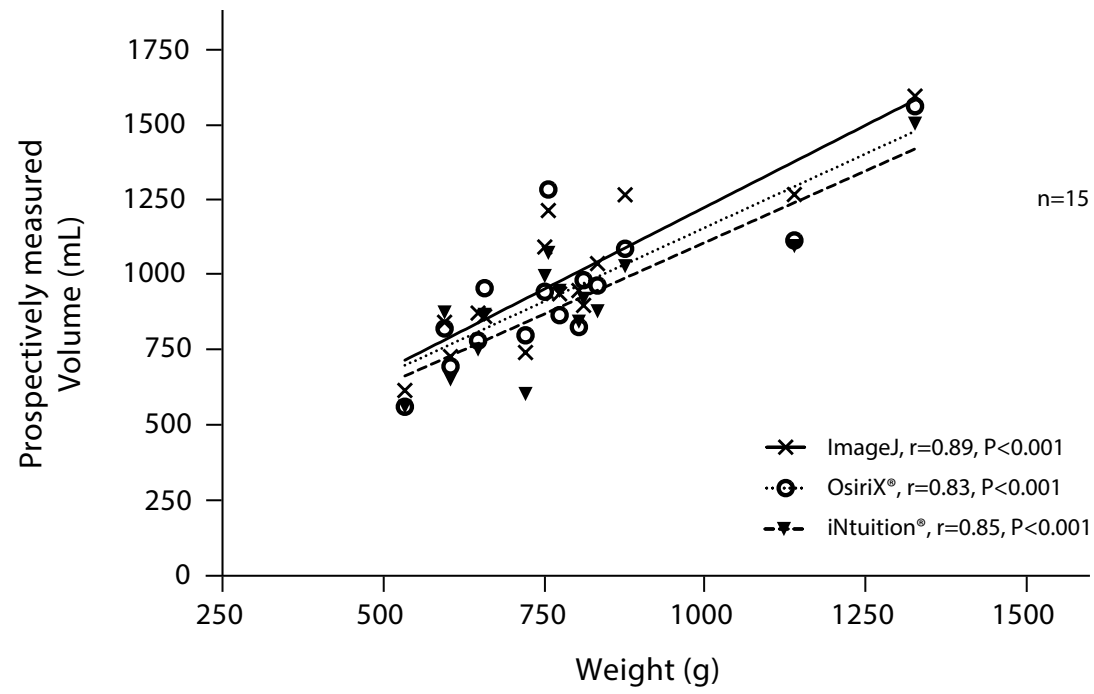

Fig. 1 Correlation between volume measured prospectively with ImageJ, OsiriX, and iNtuition and the resected specimen's weight measured in the operating theater 


\section{TIMETO PERFORM MEASUREMENT AND EFFECT OF SLICETHICKNESS}

The time required for a single volumetric assessment of the liver with ImageJ (including total liver, tumor, resected specimen) was, on average, 20 minutes (range 15-30 minutes). There was no statistical difference between the TLVs based on 0.5 vs. $1.0 \mathrm{~cm}, 0.5 \mathrm{vs.} 1.5 \mathrm{~cm}$, and 0.5 vs. $2.0 \mathrm{~cm}$. The change in volume (calculated in percentages) was not significantly different for volumetric measurements with different slice thicknesses. For a slice thickness of 1.0 or $1.5 \mathrm{~cm}, 80 \%$ of the measurements were within the $5 \%$ limit of acceptance for the TLV change. When using the $2.0 \mathrm{~cm}$ slice thickness, only $60 \%$ of the measurements were within this limit. The mean gain of time (calculated in percentages) by increasing slice thickness was $50 \%(0.5$ vs. $1.0 \mathrm{~cm}), 64 \%$ ( 0.5 vs. $1.5 \mathrm{~cm})$, and $72 \%$ (0.5 vs. $2.0 \mathrm{~cm}$ ).

\section{IMAGEJ VERSUS OSIRIX AND INTUITION}

There was a highly significant correlation between the volumes calculated with ImageJ and the volumetric analysis using OsiriX $(r=0.95, P<0.001)$. There was also a strong correlation between the volumes calculated with ImageJ and the volumetric analysis performed by a radiologist using radiology image analysis software linked to radiology hardware $(r=0.93$, $\mathrm{P}<0.001)$.
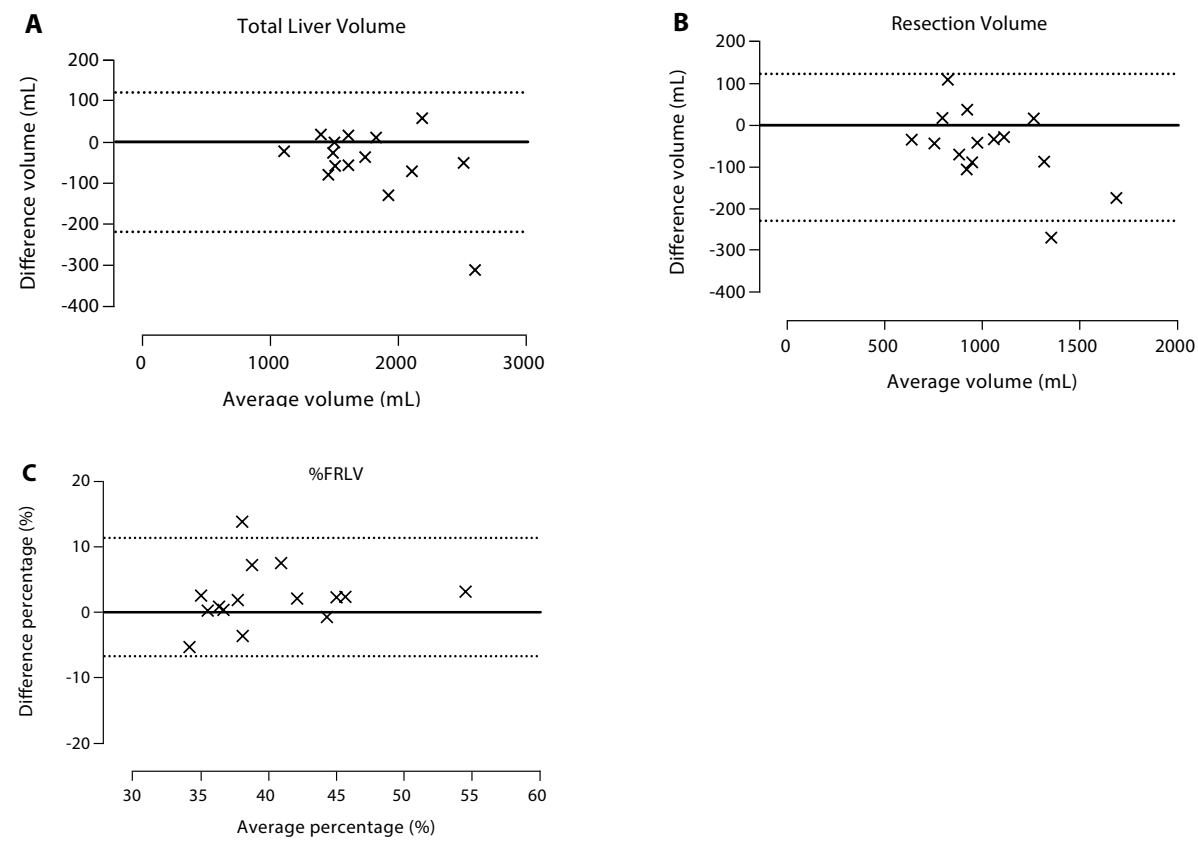

Fig.2 a. Bland-Altman analysis for interobserver variability of outlining the total liver volume with ImageJ. Horizontal solid line indicates the mean average between the two users. Horizontal dashed lines indicate $95 \%$ limits of agreement (mean \pm 1.96 SD). b. Bland-Altman analysis for interobserver variability of outlining the resectional liver volume with ImageJ. c. Bland-Altman analysis for interobserver variability of the \%functional remnant liver volume (\%FRLV) with ImageJ. 


\section{DISCUSSION}

The present study shows that prospective CT volumetric analysis of the liver in patients undergoing right hemihepatectomy with ImageJ and OsiriXon the surgeon's PC is valid and reliable. In this study, we found a strong significant correlation between resection weight and resection volume measured prospectively with ImageJ and OsiriX. Practical advantages of ImageJ and OsiriX were described previously by our group [11, 12]. In our center, the radiologists use the commercial image analysis software package iNtuition for liver CT volumetry. There was a significant correlation between the resection weight and the resection volume measured prospectively with iNtuition. There were no significant differences in the mean volumes between the three software packages. There was a strong correlation between the volumes measured with radiology software iNtuition and the volumes measured with ImageJ and OsiriX. This indicates that ImageJ and OsiriX even if operated by nonradiologists, are at least as appropriate for CT volumetry of the liver as professional radiology software. ImageJ and OsiriX bring preoperative liver CT volumetry within the surgeon's reach, independent of the support of a radiologist. Hepatopancreatobiliary (HPB) surgeons should routinely perform $\mathrm{CT}$ volumetry during the preoperative assessment of patients undergoing a major liver resection. This is especially true for patients with compromised liver function due to neoadjuvant chemotherapy, cirrhosis, or steatosis or for patients scheduled for an extended liver resection.

As we and others concluded before, the time required to perform CT volumetry of the liver can be reduced by increasing slice thickness, thereby diminishing the number of CT slices that have to be outlined[11][[17]. Data from the current study show that to make a rough estimate of the FRLV (patients with a percentage FRLV of C30\%) a slice thickness of $1.0 \mathrm{~cm}$ provides an optimal balance between accuracy and time efficiency. However, in patients with a marginally functional liver remnant volume (20-30\%) greater accuracy is needed. In these patients, it is better to use a smaller slice thickness to avoid over-or underestimating the volume. Time required to outline all ROIs is approximately 20 minutes with both ImageJ and OsiriX. However, OsiriX has a significant advantage because it has an integrated picture archiving and communication system (PACS system) in which the data (CT scans) of all examined patients are automatically stored. Additionally, OsiriX is user-friendlier, and ROIs can be selected semiautomatically, which saves time and lessens the workload [12].

Interobserver variability for ImageJ between the two users for volumetric assessment of the total and resected liver volumes was low. Bland-Altman plots revealed that there was one outperformer in the measurements of the total liver volume and the resection specimen volume. This resulted in relatively wide confidence intervals. However, it is mainly the \%FRLV that is crucial. It has been shown elsewhere that to perform liver resection safely \%FRLV has to be at least $26.6 \%$ in patients with normal liver parenchyma [4]. In the present study, the 
mean difference of the \%FRLV measurements between both ImageJ users of $2.3 \pm 4.6 \%$ was clinically acceptable because ImageJ users 1 and 2 predicted \%FRLVs of $41.4 \pm 1.6 \%$ and $39.0 \pm 1.4 \%$, respectively. In other words, the resection volume differences between the two ImageJ users should have had no clinical consequences. In patients in whom the preoperative liver volume assessment shows that the reported FRLV\% approaches $26.6 \%$, extra care is recommended.

A possible limitation of estimating the future remnant liver volume with CT volumetry is the systematic overestimation of the liver volumes compared to the resected specimens' weight. A physical density $<1.0 \mathrm{~g} / \mathrm{ml}$ and systematic overestimation of the liver volume in the present study was in accordance with those of previous studies [7, 10-12]. Inaccurate estimation of the resected specimen volume by $\mathrm{CT}$ volumetry can occur because the liver on a CT scan is in a perfused state and the postoperative resection specimen is in an unperfused state. This applies to every radiologic application, and therefore some studies have suggested the implementation of conversion factors to improve volumetric accuracy [7, $18,19]$. These factors are mostly based on the intraoperative resection volume deter-mined with the use of Archimedes principle. Unfortunately, this approach does not determine the influence of the blood content of the resection specimen. It might be that these conversion factors can lead to a more precise volumetric analysis of the liver, but their exact role remains to be determined. Patients with steatosis have more fat in the liver, and the relative densitie of fat is $0.9 \mathrm{~g} / \mathrm{ml}$. Hence, the volume is larger and the weight less than that of a normal liver. Ultimately, this changes the volume/weight standard and can also contribute to systemic overestimation.

In all right hemihepatectomies in the present study, virtual transection followed Cantlie's line, and thus the middle hepatic vein (MHV) was used as a guide for prospective volume measurements. During surgery, the demarcation line after selective inflow occlusion is used for transection and may leave liver parenchyma outside (i.e., to the right of) the MHV in some patients. As a consequence, preoperative estimation of the future resected specimen volume may be overestimated in some individuals. In line with this, Karlo et al. showed in a retrospective study that, despite a highly accurate definition of the transection line on preoperative CT or MRI scans using anatomic landmarks on postoperative MRI scans, the resection specimen volume was still overestimated [7]. Systematic overestimation, however, does not affect the functional remnant liver volume as a percentage of the total functional liver volume, which is one of the most important indicators of the risk of postoperative liver failure. 


\section{CONCLUSION}

User-friendly, easily accessible, free, downloadable software packages such as ImageJ and OsiriX can be used reliably for prospective CT volumetry of resected liver specimens. The performance of ImageJ and OsiriX is comparable to that of radiology software iNtuition. OsiriX is the most efficient and practical software package, but it can be used only on a Macintosh PC. For a Windows-based PC, ImageJ is a good alternative. 


\section{REFERENCES}

1. Shah, S.A., et al., Surgical resection of hepatic and pulmonary metastases from colorectal carcinoma. J Am Coll Surg, 2006. 202(3): p. 468-75.

2. Fusai, G. and B.R. Davidson, Management of colorectal liver metastases. Colorectal Dis, 2003. 5(1): p. 2-23.

3. Scheele, J., et al., [Resection of colorectal liver metastases. What prognostic factors determine patient selection?]. Chirurg, 2001. 72(5): p. 547-60.

4. Schindl, M.J., et al., The value of residual liver volume as a predictor of hepatic dysfunction and infection after major liver resection. Gut, 2005. 54(2): p. 289-96.

5. Shoup, M., et al., Volumetric analysis predicts hepatic dysfunction in patients undergoing major liver resection. J Gastrointest Surg, 2003. 7(3): p. 325-30.

6. Jarnagin, W.R., et al., Improvement in perioperative outcome after hepatic resection: analysis of 1,803 consecutive cases over the past decade. Ann Surg, 2002. 236(4): p. 397-406; discussion 406-7.

7. Karlo, C., et al., CT- and MRI-based volumetry of resected liver specimen: comparison to intraoperative volume and weight measurements and calculation of conversion factors. Eur J Radiol, 2010. 75(1): p. e107-11.

8. Rasband, W.S., ImageJ 1.33 edn. . U.S. National Institutes of Health, Bethesda, MD, 1997-2005.

9. Kishi, Y., et al., Three hundred and one consecutive extended right hepatectomies: evaluation of outcome based on systematic liver volumetry. Ann Surg, 2009. 250(4): p. 540-8.

10. Wigmore, S.J., et al., Virtual hepatic resection using three-dimensional reconstruction of helical computed tomography angioportograms. Ann Surg, 2001. 233(2): p. 221-6.

11. Dello, S.A., et al., Liver volumetry plug and play: do it yourself with ImageJ. World J Surg, 2007. 31(11): p. 221521.

12. van der Vorst, J.R., et al., Virtual liver resection and volumetric analysis of the future liver remnant using open source image processing software. World J Surg, 2010.
34(10): p. 2426-33.

13. Lu, Y., et al., Hepatic volumetry with PhotoShop in personal computer. Hepatobiliary Pancreat Dis Int, 2004. 3(1): p. 82-5.

14. Tu, R., et al., Assessment of hepatic functional reserve by cirrhosis grading and liver volume measurement using CT. World J Gastroenterol, 2007. 13(29): p. 3956-61.

15. Dejong, C.H.C. and O.J. Garden, Neoplasms of the liver. In: Majid AA, Kingsnorth A, eds. Advanced surgical practice. London, United Kingdom: Greenwich Medical Media, 2003: 46-56.

16. Bland, J.M. and D.G. Altman, Comparing methods of measurement: why plotting difference against standard method is misleading. Lancet, 1995. 346(8982): p. 1085-7. 17. Reiner, C.S., et al., Preoperative liver volumetry: how does the slice thickness influence the multidetector computed tomography- and magnetic resonance-liver volume measurements? J Comput Assist Tomogr, 2009. 33(3): p. 390-7.

18. Lemke, A.J., et al., Living donor right liver lobes: preoperative $\mathrm{CT}$ volumetric measurement for calculation of intraoperative weight and volume. Radiology, 2006. 240(3): p. 736-42.

19. Hwang, S., et al., Correlation of blood-free graft weight and volumetric graft volume by an analysis of blood content in living donor liver grafts. Transplant Proc, 2002. 34(8): p. 3293-4. 


\section{IMPROVING THE OUTCOME OF LIVER SURGERY: MODIFY OPERATIONS AND MODIFY PERIOPERATIVE CARE}

INITIAL EXPERIENCE WITH A MULTIMODAL ENHANCED RECOVERY PROGRAMME IN PATIENTS UNDERGOING LIVER RESECTION

R.M. van Dam, P.O. Hendry, M.M.E. Coolsen, M.H.A. Bemelmans, K. Lassen, A. Revhaug, K.C.H. Fearon, O.J. Garden and C.H.C. Dejong, on behalf of the Enhanced Recovery After Surgery (ERAS) Group. British Journal of Surgery 2008, 95:969-975

\section{THE EFFECT OF A MULTIMODAL FAST-TRACK PROGRAMME ON OUT-} COMES IN LAPAROSCOPIC LIVER SURGERY: A MULTICENTRE PILOT STUDY

J.H. Stoot, R.M. van Dam, O.R. Busch, R. van Hillegersberg, M. De Boer, S.W.M. Olde Damink, M.H. Bemelmans and C.H.C. Dejong, on behalf of the Enhanced Recovery After Surgery (ERAS) Group. HPB 2009, 11(2) 140-144

THE ORANGE TRIAL. RANDOMIZED CLINICAL TRIAL OF LAXATIVES AND ORAL NUTRITIONAL SUPPLEMENTS WITHIN AN ENHANCED RECOVERY AFTER SURGERY PROTOCOL FOLLOWING LIVER RESECTION

P. O. Hendry, R.M. van Dam, S.F. F.W. Bukkems, D.W. McKeown, R.W. Parks, T. Preston,

C.H.C. Dejong, O. J.Garden and K. C.H. Fearon, on behalf of the Enhanced Recovery After Surgery (ERAS) Group. British Journal of Surgery 2010; 97: 1198-1206

\section{A SURVEY IN THE HEPATOPANCREATOBILIARY COMMUNITY ON WAYS TO ENHANCE PATIENT RECOVERY}

E.M. Wong-Lun-Hing, T.M. Lodewick, J.H.M.B. Stoot, M.H.A. Bemelmans, S.W.M. Olde Damink, C.H.C. Dejong, R.M. van Dam. HPB 2012, 14, 818-827

THE ORANGE II TRIAL PROTOCOL. International RCT on open versus laparoscopic left lateral hepatic sectionectomy within an enhanced recovery ERAS programme R.M. van Dam E.M. Wong-Lun-Hing, G.J.P. van Breukelen, J.H.M.B. Stoot, J.R. van der Vorst, M.H.A. Bemelmans, S.W.M. Olde Damink, K. Lassen, C.H.C. Dejong and ORANGE II Study Group Trials 2012, http://www.trialsjournal.com/13/1/54

ORANGE II PLUS-TRIAL PROTOCOL. Internatinal RCT on open versus laparoscopic hemi-hepatectomy within an enhanced recovery ERAS programme E.M. Wong-Lun-Hing, A. Laurent, I. Dagher, O. Scatton, O. Soubrane, U.P. Neumann, B. Topal, R. Troisi, L. Aldrighetti, J.N. Primrose, N.W. Pearce, M. Abu Hilal, D.P. Berry, G.J.P. van Breukelen, C.H.C. Dejong, R.M. van Dam and ORANGE II Study Group. Trials 2013, submitted 

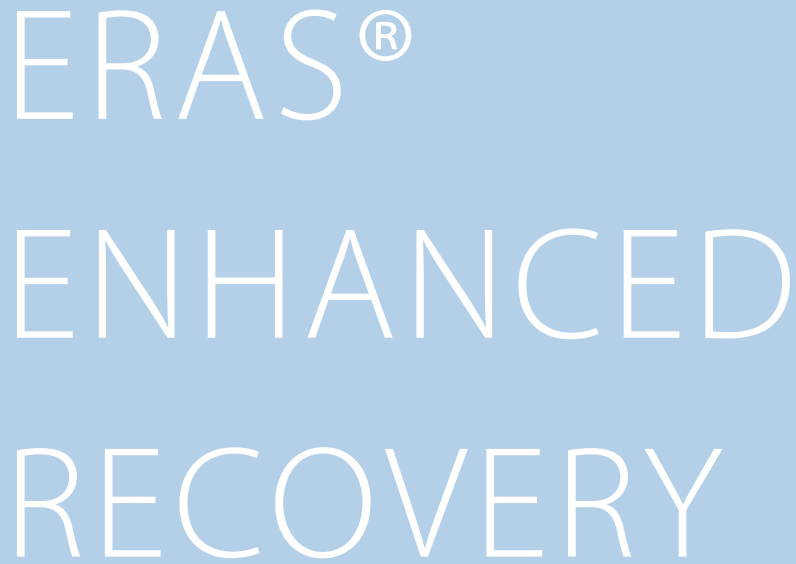

\section{PUBLISHED AS}

Initial experience with a multimodal enhanced recovery programme in patients undergoing liver resection

R.M. van Dam P.O. Hendry, M.M.E. Coolsen, M.H.A. Bemelmans, K. Lassen, A. Revhaug, K.C.H. Fearon, O.J. Garden and C.H.C. Dejongo, on behalf of the Enhanced Recovery After Surgery (ERAS) Group

British Journal of Surgery 2008, 95:969-975

DOI: dx.doi.org/10.1002/bjs.6227 


\section{ABSTRACT}

\section{BACKGROUND}

Accelerated recovery from surgery has been achieved when patients are managed within a multimodal Enhanced Recovery After Surgery (ERAS) protocol. This study evaluated the benefit of an ERAS programme for patients undergoing liver resection.

\section{METHODS}

The ERAS protocol of epidural analgesia, early oral intake and early mobilization was studied prospectively in a consecutive series of 61 patients. Outcomes were compared with those in a consecutive series of 100 patients who underwent liver resection before the start of the study. Endpoints were postoperative length of hospital stay, postoperative resumption of oral intake, readmissions, morbidity and mortality.

Fifty-six patients (92 per cent) in the ERAS group tolerated fluids within $4 \mathrm{~h}$ of surgery and a normal diet on day 1 after surgery. Median hospital stay, including readmissions, was 6.0days compared with 8.0 days in the control group $(P<0.001)$. There were no significant differences in rates of readmission (13 and 10.0 per cent respectively), morbidity (41 and 31.0 per cent) and mortality ( 0 and 2.0 per cent) between ERAS and control groups.

\section{CONCLUSION}

The ERAS fast-track protocol is safe and effective for patients undergoing liver resection. It allows early oral intake, promotes faster postoperative recovery and reduces hospital stay 


\section{INTRODUCTION}

In the past two decades, the application of multimodal perioperative care protocols, commonly referred to as fast-track or enhanced recovery programmes, has achieved significant improvements in the outcome of a variety of surgical procedures.

By targeting factors that delay postoperative recovery (pain, gut dysfunction and immobility), and combining a series of interventions to reduce perioperative stress and organ dysfunction, such programmes have been shown to accelerate postoperative recovery and reduce length of hospital stay. This can be achieved with an unaltered or even reduced rate of postoperative complications[1]. Protocols based on postoperative pain control through continuous mid-thoracic epidural anaesthesia, stimulation of gut motility, early physical reactivation, and limited use of catheters, tubes and drains have resulted in improved convalescence for many surgical procedures, including colonic surgery, open aortic aneurysm repair and oesophageal surgery[2-7]. In the past 5 years, the protocol in elective colonic surgery established by the Enhanced Recovery After Surgery (ERAS) Group has led to a reduction in median hospital stay from 9 to 5 days[6].

Within a traditional perioperative care pathway median hospital stay following liver surgery ranges from 8 to 14 days[8-11]. Morbidity is reasonably predictable, with haemorrhagic complications occurring predominantly during surgery or in the early postoperative phase, and biliary complications, intra-abdominal abscess or liver failure in the later postoperative phase $[12,13]$. It was hypothesized that application of an enhanced recovery programme for patients undergoing liver surgery would result in accelerated recovery and a shorter hospital stay. The main aim of the present study was to test the feasibility, safety and effectiveness of an ERAS protocol in patients undergoing liver surgery in two tertiary referral liver units in Europe.

\section{METHODS}

The ERAS liver study was initiated within the collaborative ERAS Group, and conducted in two units that perform high-volume liver surgery. The study was a prospective case series implementation study comparing outcomes with those in a historical control group.

The ERAS patient group comprised a consecutive series of 61 patients admitted to either the liver unit of Maastricht University Medical Center, The Netherlands, between 15 February 2005 and 31 August 2006, or the liver unit of the Royal Infirmary of Edinburgh, UK, where patient recruitment started on 1 February 2006. The control group consisted of a consecutive series of 100 patients who had liver resection in one of the two units between 1 July 2003 
and 31 December 2004 (50 consecutive patients from each unit), when a more traditional care pathway was still in use. Patient and outcome data were derived from a prospectively collected database of all liver resections performed since 1 January 2000 in both units. In both groups, patients undergoing elective liver resection for primary or secondary tumours were considered eligible for the study if they had normal underlying liver function. Patients were excluded if they required biliary reconstruction or had emergency surgery.

In both units, the same group of liver surgeons performed the liver resections in the ERAS group as well as in the control group. In Maastricht more fellows with hepatopancreatobiliary training operated in the ERAS group. Bilateral subcostal transverse abdominal incisions were used. The transection plane was determined by intraoperative ultrasonography. To avoid excessive blood loss central venous pressure was reduced to below $5 \mathrm{mmHg}$ during transection using the Cavitron Ultrasonic Surgical Aspirator (CUSA; Valleylab, Boulder, Colorado, USA) and argon beam coagulation. The portal pedicles supplying and veins draining the sectors to be resected were divided and ligated with a running polypropylene suture. After removal of the liver specimen, the raw surface of the liver remnant was subjected to argon beam coagulation and sealed with TachoSil (Nycomed, Zurich, Switzerland). In accordance with the protocol, no abdominal drains were placed in the ERAS group and the abdomen was closed with a standard running suture.

Before the introduction of the ERAS programme neither of the institutions had a written, agreed perioperative care pathway. There were no specific measures to avoid prolonged preoperative and postoperative fasting, nasogastric decompression, excessive use of intravenous fluids and systemic opioids, prophylactic abdominal drains and postoperative immobilization. This conventional postoperative care programme emphasized prolonged rest for both the patient and the gastrointestinal tract.

The ERAS multimodal evidence-based recovery programme protocol, which was designed originally for elective colonic surgery, was modified to cover all aspects of elective liver resection[2]. Details of the ERAS liver protocol are given in Table 1. 


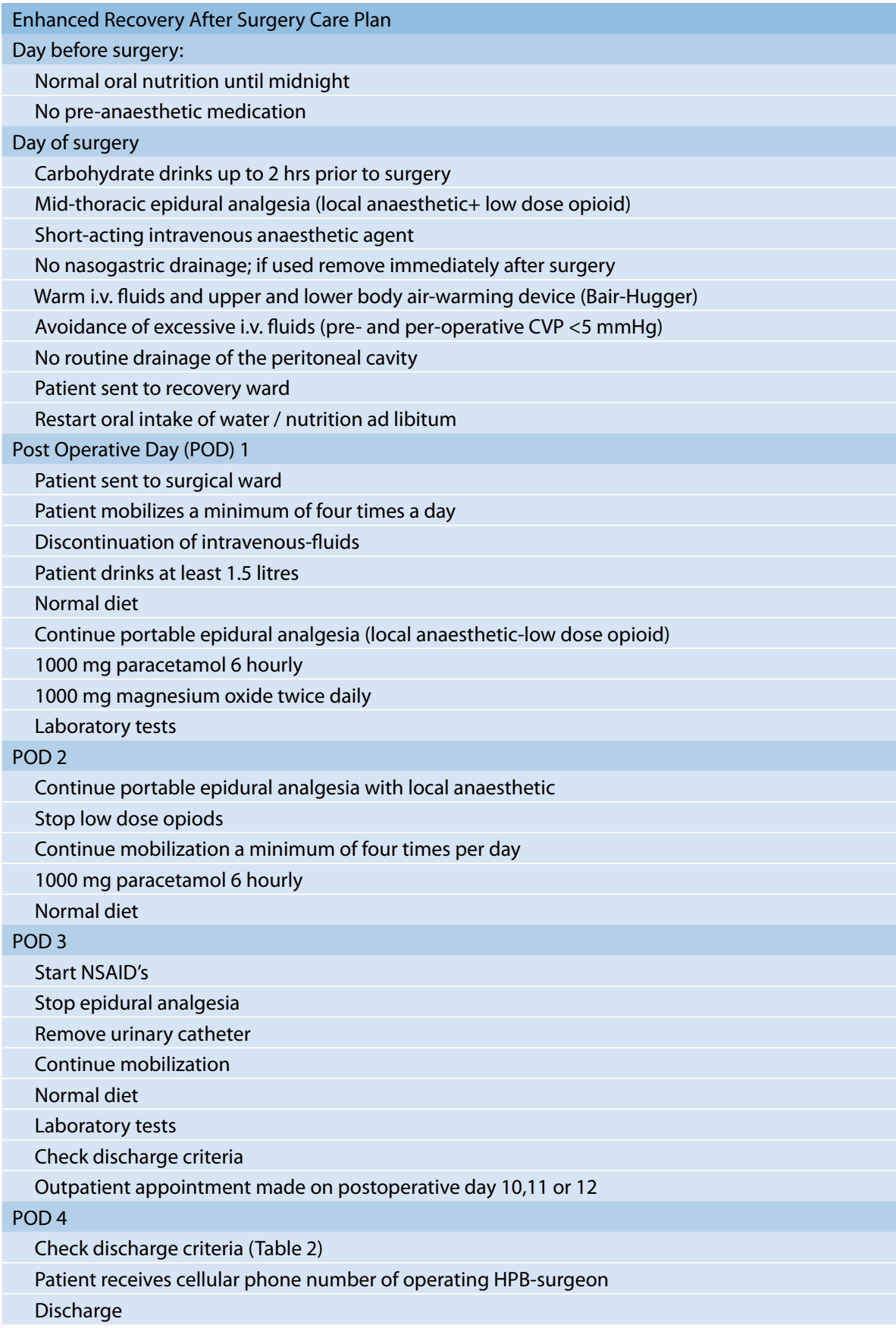


In both groups, data were obtained prospectively during the hospital stay and for 90 days after surgery. Data recorded for each patient included morbidity, defined as complications related to the liver surgery, mortality, readmissions, postoperative hospital stay, use of preoperative chemotherapy, duration of surgical procedure, blood loss, anaesthetic agents used and type of hepatectomy, defined according to the Brisbane 2000 terminology[14]. In the ERAS group, data on location of the epidural catheter, removal of the nasogastric tube if used, and time to resumption of oral intake and mobilization were also noted.

The primary endpoint of the study was total length of hospital stay, defined as the number of nights spent in hospital, including nights after readmission within 30 days after surgery. To facilitate objective assessment of clinical outcome and to prevent discharge too soon, predefined discharge criteria were used in the ERAS group (Table 2).

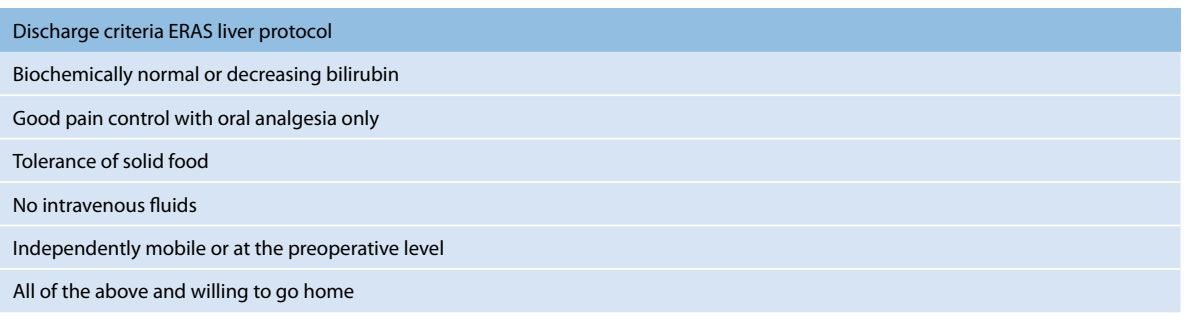

Table 2 Discharge criteria in Enhanced Recovery After Surgery liver protocol

Patients were informed about the protocol at a preadmission counselling session, where the importance of early mobilization and early oral intake were explained. Patients were discharged only if they met the discharge criteria and follow-up within 3 days was possible in the outpatient clinic. Patients were given the mobile telephone number of the operating consultant surgeon to allow direct communication and safe deployment of the protocol. 


\begin{tabular}{|c|c|c|}
\hline & $\begin{array}{l}\text { ERAS } \\
(n=61)\end{array}$ & $\begin{array}{l}\text { Control } \\
(n=100)\end{array}$ \\
\hline Median (range) age (years) & $62(24-82)$ & $60(20-81)$ \\
\hline Sex ratio (M:F) & $35: 26$ & $51: 49$ \\
\hline \multicolumn{3}{|l|}{ ASA grade } \\
\hline I & $11(18)$ & $14(14-0)$ \\
\hline ॥ & $42(69)$ & $64(64-0)$ \\
\hline III & $8(13)$ & $22(22-0)$ \\
\hline \multicolumn{3}{|l|}{ Liver pathology } \\
\hline Colorectal metastasis & $51(84)$ & $72(72-0)$ \\
\hline Other metastasis & $2(3)$ & $4(4-0)$ \\
\hline $\begin{array}{l}\text { Hepatocellular carcinoma or } \\
\text { cholangiocarcinoma }\end{array}$ & $4(7)$ & $9(9-0)$ \\
\hline Benign lesion & $4(7)$ & $14(14-0)$ \\
\hline T4 invasive tumours in liver & $0(0)$ & $1(1-0)$ \\
\hline
\end{tabular}

Table 3 Patient demographics. Values in parentheses are percentages unless indicated otherwise. ERAS, Enhanced Recovery After Surgery; ASA, American Society of Anesthesiologists.

Patients in the control group were discharged without formal discharge criteria. The following secondary endpoints were evaluated: resumption of oral intake, defined as oral intake of water or normal food without discontinuation for at least $24 \mathrm{~h}$, readmissions, total morbidity, death and length of primary hospital stay, defined as the number of nights spent in hospital after surgery, excluding readmissions. Complications were registered during the primary admission or at subsequent outpatient clinic visits, after at least 6 and 30 days, using departmental liver registry databases.

\section{STATISTICAL ANALYSIS}

Continuous data are expressed as median (range). Hospital stay, time to resumption of oral intake and postoperative duration of epidural analgesia were analysed using the MannWhitney $\mathrm{U}$ test. Readmission, complication and mortality rates were analysed using $\mathrm{X} 2$ test or Fisher's exact test. $\mathrm{P}<0.050$ was considered to be statistically significant. Data were analysed using SPSS version 13.0 for Windows (SPSS, Chicago, Illinois, USA). 


\begin{tabular}{llll} 
Types of liver resection & ERAS & \\
& $\begin{array}{l}\text { N }=61 \\
\text { Hemihepatectomy }\end{array}$ & $20(32.8 \%)$ & $\begin{array}{c}\text { Controls } \\
\mathrm{N}=100\end{array}$ \\
\hline Hemihepatectomy $+\geq 1$ metastasectomies & $6(9.8 \%)$ & $38(38.0 \%)$ \\
\hline Extended hemihepatectomy & $7(11.5 \%)$ & $16(16.0 \%)$ \\
\hline Multiple $(\geq 2)$ segmentectomy & $17(27.9 \%)$ & $3(3 \%)$ \\
\hline Central resection / trisegmentectomy & $1(1.6 \%)$ & $18(18.0 \%)$ \\
\hline Metastasectomy or mono-Segmentectomy & $10(16.4 \%)$ & $4(4.0 \%)$ \\
\hline Repeat-hepatectomy \% & $7(11.5 \%)$ & $21(21.0 \%)$ \\
\hline & & $5(5 \%)$ \\
\hline
\end{tabular}

Table 4 Types of liver resection

\section{RESULTS}

Patient characteristics were similar in the ERAS and control groups (Table 3). Types of liver resection are shown in Table 4. A higher proportion of patients in the ERAS group had repeat liver resections ( 11 per cent versus 5.0 per cent in control group; $P=0.214$ ) and resections after intensive chemotherapy (62 versus 33.0 per cent; $\mathrm{P}<0.001$ ). The median (range) duration of surgery was 220 (60-420) min in the ERAS group and 270 (106-510) $\mathrm{min}$ in the control group $(P<0.001)$, and blood loss during surgery was $750(0-5000)$ and $800(0-6000)$ $\mathrm{ml}$ respectively $(P=0.758)$. Abdominal drains were used significantly less often in the ERAS group (2 versus 66.0 per cent; $P<0.001$ ).

In Edinburgh, the use of a nasogastric decompression tube in patients having partial liver resection had already been discontinued before 1 July 2003. In Maastricht, nasogastric decompression tubes were still used in most control patients. A nasogastric tube was inserted at induction in 37 of 47 patients in the ERAS group, significantly less frequently than in the control group (96 per cent) $(P=0.013)$. In the ERAS group, the nasogastric tube was removed at the end of surgery or in the recovery ward within $4 \mathrm{~h}$ of surgery. Oral intake was resumed within $4 \mathrm{~h}$ after surgery in 56 patients (92 per cent) in the ERAS group. Two patients required reinsertion of a nasogastric tube. The median (range) time to successful resumption of normal diet was $1(0-3)$ day in the ERAS group, compared with 3 (0-14) days in controls $(\mathrm{P}<0.001)$. Three patients in the ERAS group (5 per cent) became constipated after postoperative day 3 , despite initial tolerance of normal food, but this resolved within 5 days by restricting intake.

In the ERAS group, 58 patients (95 per cent) followed the anaesthetic protocol of mid-thoracic epidural analgesia commencing before surgery $(0.1$ per cent bupivacaine $+2 \mu \mathrm{g} / \mathrm{ml}$ fentanyl) with maintenance isoflurane or sevoflurane inhalation anaesthesia, or intravenous propofol, as described previously[15]. After operation epidural analgesia comprising $0 \cdot 1$ per cent bupivacaine and $2 \mu \mathrm{g} / \mathrm{ml}$ fentanyl was administered for at least 3 days via a portable pa- 
tient-controlled device (GemStar Infusion Systems; Hospira, Lake Forest, Illinois, UK) at a rate of 4-10 $\mathrm{ml} / \mathrm{h}$, combined with oral non-opioid analgesics. This differed from anaesthesiological management in the control group, where 89 patients ( 89.0 per cent) received epidural analgesia combined with isoflurane or sevoflurane inhalation anaesthesia. In the postoperative period epidural analgesia consisting of $0 \cdot 1$ per cent bupivacaine $+2 \mu \mathrm{g} / \mathrm{ml}$ fentanyl was administered without a specific time schedule at a daily fixed dose of $4-10 \mathrm{ml} / \mathrm{h}$, combined with intramuscular opioids (mainly piritramide) and oral analgesics.

\begin{tabular}{|c|c|c|c|}
\hline \multicolumn{4}{|c|}{ Outcome of enhanced recovery programme in liver surgery } \\
\hline & ERAS & CONTROLS & $\mathrm{P}$ \\
\hline MAASTRICHT ONLY* & $\mathrm{N}=47$ & $\mathrm{~N}=50$ & \\
\hline Nasogastric tube $(\mathrm{NGT})^{*}$ & $37(78.7 \%)^{*}$ & $50(100 \%)^{*}$ & $\mathrm{p}<0.01^{*}$ \\
\hline $\begin{array}{l}\text { Removal of NGT on day of } \\
\text { surgery }\end{array}$ & $34 / 37(91.9 \%)^{*}$ & $0(0 \%)^{*}$ & $\mathrm{P}<0.0001^{*}$ \\
\hline MAASTRICHT \& EDINBURGH & $\mathrm{N}=61$ & $\mathrm{~N}=100$ & \\
\hline Epidural analgesia & $58(95.1 \%)$ & 89 (89 \%) & NS \\
\hline Abdominal drain & $1(1.6 \%)$ & $66(66 \%)$ & $\mathrm{p}<0.0001$ \\
\hline Complication rate & 25 (41.0\%) & $31(31.0 \%)$ & NS \\
\hline Mortality & $0(0 \%)$ & $2(2.0 \%)$ & NS \\
\hline Readmission rate & $8(13.1 \%)$ & $10(10.0 \%)$ & NS \\
\hline $\begin{array}{l}\text { Total length of stay (median, } \\
\text { range), (readmissions included) }\end{array}$ & $\begin{array}{l}6.0 \text { days } \\
(3-82)\end{array}$ & $\begin{array}{l}8.0 \text { days } \\
(4-68)\end{array}$ & $\mathrm{p}<0.001$ \\
\hline $\begin{array}{l}\text { Primary Hospital length of stay } \\
\text { (median, range) }\end{array}$ & $\begin{array}{l}6.0 \text { days } \\
(3-82)\end{array}$ & $\begin{array}{l}8.0 \text { days } \\
(4-55)\end{array}$ & $\mathrm{p}<0.0001$ \\
\hline
\end{tabular}

Postoperative epidural analgesia was continued for a median (range) of 3 (1-5) days in the ERAS group and $2(0-6)$ days in the control group $(P<0.001)$. Epidural analgesia was discontinued earlier in the control group, and intramuscular or intravenous opioids (piritramide) were used more widely.

Outcome data are summarized in Table 5. There were no deaths in the ERAS group and one patient died within 30 days in the control group. A further control patient died after 62 days. Overall morbidity rates (percentage of patients with at least one complication) were 41 and 31.0 per cent in the ERAS and control groups respectively ( $P=0.197)$ (Table 6). All complications in the ERAS group were managed by non-surgical means. Readmission rates in ERAS and control groups were similar (13 versus 10.0 per cent respectively; $\mathrm{P}=0.610$ ).

Fifty-two ( 85 per cent) of 61 patients in the ERAS group were completely mobile on the third day after operation and 29 (48 per cent) were discharged from hospital within 5 days. Median (range) total postoperative hospital stay, including readmissions, was 6.0 (3-82) days in the ERAS group and 8.0 (4-68) days in the control group ( $P<0.001)$ (Fig. 1). 


\begin{tabular}{|c|c|c|c|}
\hline \multicolumn{4}{|c|}{ Complications of enhanced recovery programme in liver surgery } \\
\hline \multirow[b]{3}{*}{ Complications } & \multirow{3}{*}{$\begin{array}{l}\text { ERAS } \\
\mathrm{N}=61 \\
41.0 \% \text { of patients }\end{array}$} & \multirow{3}{*}{$\begin{array}{l}\text { Historical } \\
\mathrm{N}=100 \\
31.0 \% \text { of patients }\end{array}$} & \multirow{3}{*}{$\begin{array}{l}\text { P- } \\
\text { Value } \\
\text { NS }\end{array}$} \\
\hline & & & \\
\hline & & & \\
\hline Bile leak & $4(6.6 \%)$ & $4(4.0 \%)$ & NS \\
\hline Liver failure & $3(4.9 \%)$ & $1(1.0 \%)$ & NS \\
\hline Sepsis & $0(0.0 \%)$ & $1(1.0 \%)$ & NS \\
\hline Abdominal abscess & $5(8.2 \%)$ & $7(7.0 \%)$ & NS \\
\hline Ileus & $3(4.9 \%)$ & $1(2.5 \%)$ & NS \\
\hline Pneumonia & $3(4.9 \%)$ & $2(2.0 \%)$ & NS \\
\hline Pleural Effusion & $2(3.3 \%)$ & $2(2.0 \%)$ & NS \\
\hline Pulmonary Embolism & $0(0 \%)$ & $1(1.0 \%)$ & NS \\
\hline Myocardial Infarction & $2(3.3 \%)$ & $3(3.0 \%)$ & NS \\
\hline Wound infection & $9(14.8 \%)$ & $5(5.0 \%)$ & $p<0.04$ \\
\hline Other minor & $3(4.9 \%)$ & $16(20.0 \%)$ & $\mathrm{p}<0.02$ \\
\hline
\end{tabular}

Table 6 Complications

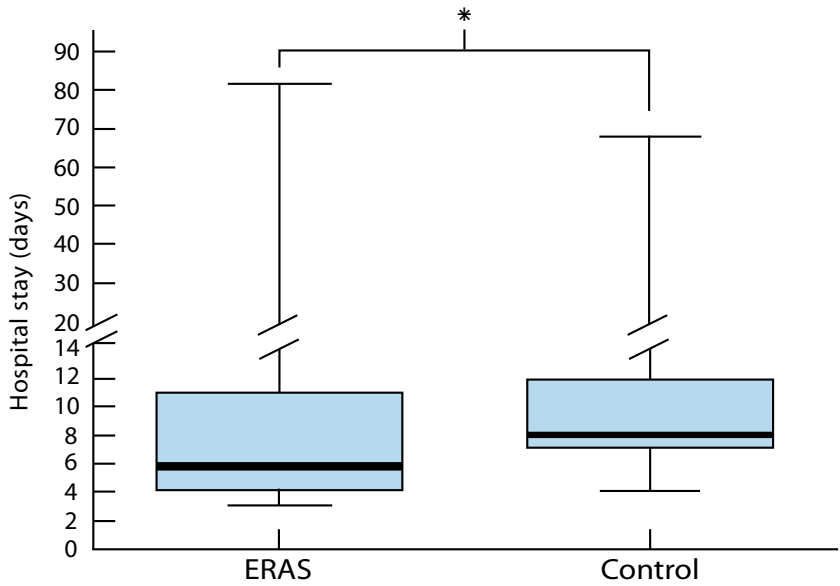

Fig. 1 Total length of hospital stay, including readmissions, in Enhanced Recovery After Surgery (ERAS) and control groups. Horizontal lines within boxes, boxes and error bars represent median, interquartile range and total range respectively. $\mathrm{P}<0.001$ (Mann-Whitney $\mathrm{U}$ test) 


\section{DISCUSSION}

The present study showed that use of an evidence-based multimodal enhanced recovery programme after liver resection accelerated postoperative recovery and led to a significantly shorter hospital stay. Patients managed according to the ERAS protocol were able to drink fluids within $4 \mathrm{~h}$ of liver resection and eat normal food on the day after surgery. Most patients were completely mobile after 3 days and, as a consequence, almost half of the patients were discharged from hospital within 5 days. Median total hospital stay (including readmissions) was reduced from 8 to 6 days, which is considerably shorter thanthe8-14 days reported by other European centres[8-10]. Application of the ERAS protocol did not have any detrimental effect on safety in liver resection.

Based on previous experience of enhanced recovery implementation studies within these units, and attempts to randomize between fast-track and traditional care in a multicentre abdominal surgery trial[6, 16], a randomized clinical trial comparing fast-track and traditional care was considered impractical. Both units had implemented ERAS protocols over the previous few years and, in view of the growing evidence base for such programmes, a traditional perioperative protocol was felt to represent suboptimal care. More importantly, it would have been necessary for nursing and surgical staff to return to traditional perioperative care pathways. Therefore, this feasibility study was designed as a prospective case series with a historical control group.

Several important issues have become apparent from this study. Importantly, adverse outcomes, assessed in terms of readmissions, complications and deaths, were similar in the ERAS and control groups. The complication rate in the ERAS group is in keeping with recently published rates of 23-52 per cent[10, 17-20]. Although the morbidity rate appeared to be higher in the ERAS group, the study was not sufficiently powered to determine statistical differences in the incidence of complications. Moreover, complications directly related to liver surgery, such as bleeding, bile leakage or temporary liver failure, are unlikely to be influenced by postoperative care. Avoidance of abdominal drains in this study might arguably have influenced the complication rate, but the authors consider this unlikely because the incidence of bile leak and intra-abdominal abscess was similar in the two groups. Prophylactic drains are employed in many centres to detect early complications such as postoperative haemorrhage or bile leakage, to remove intraperitoneal fluids and to prevent abscess formation. However, there is evidence that abdominal drainage after liver resection does not reduce the incidence of reinterventions for these complications[21-23]. Some studies have even noted higher rates of infected collections when drainage was used[13, 21, 24]. This suggests that drains might be detrimental to clinical outcome by providing a route for ascending infections. Another disadvantage of drains within an enhanced recovery setting is that they represent a significant impediment to achieving early mobilization. 
A few elements have proven difficult to implement universally. The use of carbohydrate drinks before surgery reduces thirst, hunger and anxiety, and is safe up to $2 \mathrm{~h}$ before abdominal surgery[25]. However, a preoperative fasting policy of 'nil by mouth from midnight' is still common. Owing to tradition in the anaesthesia department nasogastric decompression tubes were still used extensively during surgery in both groups in Maastricht, but were removed immediately after surgery in the ERAS group. This practice meant a major change in clinical routines, and did not appear to result in more pulmonary complications in the present study. In general, nasogastric decompression is still used widely following abdominal surgery, despite evidence that it is unnecessary and can result in more pulmonary complications[26]. Further support for avoidance of nasogastric tubes specifically in liver surgery has been published recently[11]. Early postoperative enteral nutrition compared with 'nil by mouth' improves clinical outcome[27]. In the present study, oral intake of water within $4 \mathrm{~h}$ after surgery and institution of normal nutrition on the day after operation were well tolerated in the ERAS group. Early resumption of normal diet in combination with other elements of the enhanced recovery programme is designed to reduce the occurrence of delayed gastrointestinal function after surgery and even promote appetite. Therefore, the ability of the patient to tolerate normal food is an outcome in itself. A period of delayed gastrointestinal functioning after operation, observed in a few patients, was registered as a complication. It might also be argued that a delay in gastrointestinal functioning is a physiological phenomenon that occurs after abdominal surgery in many patients. A restrictive perioperative intravenous fluid regimen may help to reduce the occurrence of delayed gastrointestinal functioning[28]. Fluid restriction may well be an important issue in hepatic surgery and is the subject of ongoing research in the authors' units.

It is important that surgical patients are treated in an environment that encourages early mobilization[4]. Mobilization on the day of surgery was possible in Edinburgh, but was not achieved in Maastricht because the recovery unit did not contain the appropriate facilities. Adequate pain control and a substantial effort by nursing staff are required to achieve early mobilization. 


\section{CONCLUSION}

A multimodal enhanced recovery programme for patients undergoing liver resection for primary and secondary tumours, but with otherwise normal hepatic function, is feasible and effective. Patients were able to drink within $4 \mathrm{~h}$ of surgery, eat on the following day and were usually mobile by the third day after operation. This is indicative of earlier recovery and was accompanied by a 25 per cent reduction in postoperative hospital stay. The fact that only half of the patients were discharged within 5 days indicates that there is further room for improvement. 


\section{REFERENCES}

1. Kehlet, H. and J.B. Dahl, Anaesthesia, surgery, and challenges in postoperative recovery. Lancet, 2003. 362(9399): p. 1921-8.

2. Fearon, K.C., et al., Enhanced recovery after surgery: a consensus review of clinical care for patients undergoing colonic resection. Clin Nutr, 2005. 24(3): p. 466-77.

3. Podore, P.C. and E.B. Throop, Infrarenal aortic surgery with a 3-day hospital stay: A report on success with a clinical pathway. J Vasc Surg, 1999. 29(5): p. 787-92.

4. Kehlet, H. and D.W. Wilmore, Multimodal strategies to improve surgical outcome. Am J Surg, 2002. 183(6): p. 630-41.

5. Nygren, J., et al., A comparison in five European Centres of case mix, clinical management and outcomes following either conventional or fast-track perioperative care in colorectal surgery. Clin Nutr, 2005. 24(3): p. 45561.

6. Maessen, J., et al., A protocol is not enough to implement an enhanced recovery programme for colorectal resection. Br J Surg, 2007. 94(2): p. 224-31.

7. Cerfolio, R.J., et al., Fast tracking after Ivor Lewis esophagogastrectomy. Chest, 2004. 126(4): p. 1187-94.

8. Capussotti, L., et al., Randomized clinical trial of liver resection with and without hepatic pedicle clamping. $\mathrm{Br}$ J Surg, 2006. 93(6): p. 685-9.

9. Dimick, J.B., et al., National trends in the use and outcomes of hepatic resection. J Am Coll Surg, 2004. 199(1): p. 31-8.

10. Jarnagin, W.R., et al., Improvement in perioperative outcome after hepatic resection: analysis of 1,803 consecutive cases over the past decade. Ann Surg, 2002. 236(4): p. 397-406; discussion 406-7.

11. Pessaux, P., et al., Randomized clinical trial evaluating the need for routine nasogastric decompression after elective hepatic resection. Br J Surg, 2007. 94(3): p. 297303.

12. Belghiti, J., et al., Seven hundred forty-seven hepatectomies in the 1990s: an update to evaluate the actual risk of liver resection. J Am Coll Surg, 2000. 191(1): p. 38-46.

13. Burt, B.M., et al., An audit of results of a no-drainage practice policy after hepatectomy. Am J Surg, 2002. 184(5): p. 441-5.

14. Strasberg, S.M., Nomenclature of hepatic anatomy and resections: a review of the Brisbane 2000 system. J Hepatobiliary Pancreat Surg, 2005. 12(5): p. 351-5.

15. van de Poll, M.C., et al., Interorgan amino acid exchange in humans: consequences for arginine and citrulline metabolism. Am J Clin Nutr, 2007. 85(1): p. 167-72.

16. Wind, J., et al., Perioperative strategy in colonic surgery; LAparoscopy and/or FAst track multimodal management versus standard care (LAFA trial). BMC Surg, 2006. 6: p. 16

17. Petrowsky, H., et al., A prospective, randomized, controlled trial comparing intermittent portal triad clamping versus ischemic preconditioning with continuous clamping for major liver resection. Ann Surg, 2006. 244(6): p. 921-8; discussion 928-30.

18. Figueras, J., et al., Application of fibrin glue sealant after hepatectomy does not seem justified: results of a randomized study in 300 patients. Ann Surg, 2007. 245(4): p. 536-42.

19. Schroeder, R.A., et al., Predictive indices of morbidity and mortality after liver resection. Ann Surg, 2006. 243(3): p. 373-9.

20. Benzoni, E., et al., Liver resective surgery: a multivariate analysis of postoperative outcome and complication. Langenbecks Arch Surg, 2007. 392(1): p. 45-54.

21. Fong, Y., et al., Drainage is unnecessary after elective liver resection. Am J Surg, 1996. 171(1): p. 158-62.

22. Petrowsky, H., et al., Evidence-based value of prophylactic drainage in gastrointestinal surgery: a systematic review and meta-analyses. Ann Surg, 2004. 240(6): p. 1074-84; discussion 1084-5.

23. Belghiti, J., et al., Drainage after elective hepatic resection. A randomized trial. Ann Surg, 1993. 218(6): p. 748-53.

24. Liu, C.L., et al., Abdominal drainage after hepatic resection is contraindicated in patients with chronic liver 
diseases. Ann Surg, 2004. 239(2): p. 194-201.

25. Brady, M., S. Kinn, and P. Stuart, Preoperative fasting for adults to prevent perioperative complications. Cochrane Database Syst Rev, 2003(4): p. CD004423.

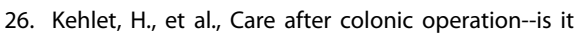
evidence-based? Results from a multinational survey in Europe and the United States. J Am Coll Surg, 2006. 202(1): p. 45-54.

27. Lewis, S.J., et al., Early enteral feeding versus "nil by mouth" after gastrointestinal surgery: systematic review and meta-analysis of controlled trials. BMJ, 2001. 323(7316): p. 773-6.

28. Lobo, D.N., et al., Effect of salt and water balance on recovery of gastrointestinal function after elective colonic resection: a randomised controlled trial. Lancet, 2002. 359(9320): p. 1812-8 


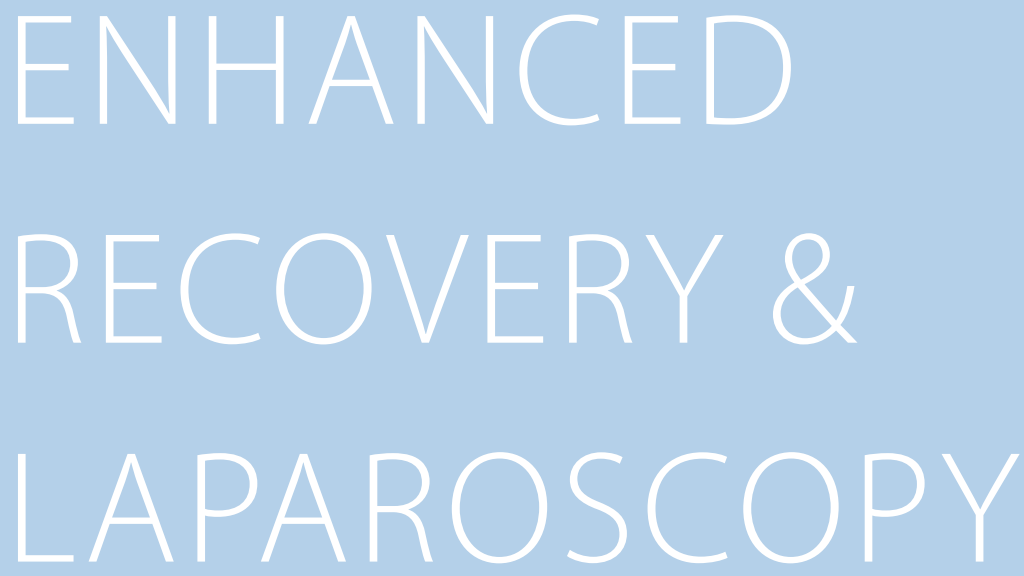

PUBLISHED AS

The effect of a multimodal fast-track programme on outcomes in laparoscopic liver surgery: a multicentre pilot study

J.H. Stoot, R.M. van Dam, O.R. Busch, R. van Hillegersberg, M. De Boer, S.W.M. Olde Damink, M.H. Bemelmans and C.H.C. Dejong, on behalf of the Enhanced Recovery After Surgery (ERAS) Group.

HPB 2009, 11 (2) 140-144

DOI: dx.doi.org/10.1111/j.1477-2574.2009.00025.x 


\section{ABSTRACT}

\section{OBJECTIVE}

This study was conducted to evaluate the added value of an enhanced recovery after surgery (ERAS) programme in laparoscopic liver resections for solid tumours.

\section{METHODS}

Patients undergoing laparoscopic liver resection between July 2005 and July 2008 were included. Indications for resections included presumed benign and malignant liver lesions. Primary outcome was total length of hospital stay (LOS). Secondary outcomes were functional recovery, complications, conversions, blood loss and duration of operation.

\section{RESULTS}

Thirteen patients were treated by laparoscopic liver resections in the ERAS programme in one centre (group 1). Their outcomes were compared with outcomes of 13 laparoscopic procedures performed either before the introduction of the ERAS programme during 2003-2005 in the same centre or during the same period in other centres using traditional care (group 2). Median total LOS was 5.0 days (range 3-10 days) in group 1 and 7.0 days (3-12 days) in group 2. This difference was not statistically significant. Functional recovery occurred 2 days earlier in group 1 (median 3.0 days [range 1-7 days] vs. median 5.0 days [range 2-8 days]; $P$ $<0.044)$. There were no significant differences in complications, conversions or duration of operation. Blood loss was significantly less in the ERAS group (median $50 \mathrm{ml}$ [range 50-200 $\mathrm{ml}$ ] vs. median $250 \mathrm{ml}$ [range $50-800 \mathrm{ml}$; $\mathrm{P}<0.002$ ).

\section{CONCLUSION}

This exploratory, multicentre, fast-track laparoscopic liver resection study is the first such study conducted. Although small, the study suggests that a multimodal enhanced recovery programme in laparoscopic liver surgery is feasible, safe and may lead to accelerated functional recovery and reductions in LOS. 


\section{INTRODUCTION}

Laparoscopic surgical procedures have been popularized because they allow for rapid recovery, shorter postoperative hospital stays and improved cosmetic outcomes. In gastrointestinal surgery, the minimally invasive approach is extensively used in many operations and has become the gold standard for some of these procedures, such as cholecystectomy and adrenalectomy[1, 2]. For solid tumours in the liver, the laparoscopic technique is used more and more frequently in expert centres and the results, mostly from cohort studies, suggest benefits, notably in terms of shorter postoperative stays[3]. With respect to improved recovery and outcome, a multimodal enhanced recovery after surgery (ERAS) programme has recently shown promising results in many open elective colorectal operations, as well as in liver surgery[4, 5]. This multimodal recovery programme is evidence-based and combines several interventions in perioperative care to reduce the stress response and organ dysfunction, with a focus on enhancing recovery $[5,6]$. The key elements of this protocol include the provision of preoperative counselling, perioperative i.v. fluid restriction, optimal pain relief, preferably without the use of opioid analgesia, early oral nutrition and enforced mobilization, and the absence of nasogastric tubes and drains[6-8]. In other fields of elective surgery, similar programmes have been shown to reduce hospital stay by several days[9, 10]. However, to our knowledge, the added value of a fast-track ERAS programme in laparoscopic liver surgery has hitherto not been explored. At the Maastricht University Medical Centre (MUMC), the ERAS programme for liver surgery started 2 years after the laparoscopic programme for left lateral sectionectomies. Therefore, a pilot study was conducted to find out whether the ERAS programme was feasible and safe and whether it improved recovery after laparoscopic liver surgery.

\section{METHODS}

Patients undergoing laparoscopic liver resections for solid liver tumours between July 2003 and July 2008 were enrolled in the study. Preoperative assessment consisted of standard plasma liver function tests, preoperative radiological evaluation with abdominal ultrasonography, contrast-enhanced computed tomography (CT) and/or selective magnetic resonance imaging (MRI) and/or CT-positron emission tomography (CT-PET). Multidisciplinary consent was also required. All lesions $<10 \mathrm{~cm}$ in diameter and judged amenable to laparoscopic resection were included (left lateral sectionectomy or anterolateral small lesions of the right side of the liver). All but one lesion were limited to lateral liver segments (II, III, V, VI). One lesion was located centrally in segment IV. Indications for surgery included presumed benign and malignant liver lesions. For patients with presumed benign disease, the indications were suspicion of a hepatocellular adenoma or cystadenoma or, in cases where the nature of the tumour was uncertain, the presence of symptoms and risk of bleeding. Indications for liver 
metastases were synchronous or metachronous liver tumours from colorectal malignancies. Patients were informed about the operative procedure and consent was obtained.

The ERAS perioperative programme was instituted in the surgical unit in Maastricht at the beginning of 2002, initially for patients undergoing elective colonic resections[11]. The protocol for this multimodal, evidence-based, enhanced recovery programme for colonic surgery requires extensive preoperative counselling, no premedication, no preoperative fasting but carbohydrate-containing liquids until 2 hours before surgery, mid-thoracic epidural anaesthesia and short-acting anaesthetics, perioperative oxygen supplementation, avoidance of perioperative fluid overload, non-opioid pain management, no routine use of nasogastric tubes, early removal of bladder catheters, standard laxatives and prokinetics, and early and enhanced postoperative feeding and mobilization.[5, 11]. This protocol was modified in 2005 to cover all aspects of liver surgery, including laboratory liver function tests, and to avoid excessive fluids (preoperative and perioperative central venous pressure [CVP] $<5 \mathrm{mmHg}$ )[4]. Until May 2005, traditional care was given before, during and after liver surgery. Therefore, the 5-year period during which laparoscopic liver surgery for solid tumours has been performed at MUMC was divided into two periods, representing the 2 years before the ERAS programme was implemented and the 3 years immediately following its implementation. As the number of patients in the MUMC control group (patients undergoing surgery with traditional perioperative care) was too small to allow us to draw firm conclusions, other major liver centres in the Netherlands, which were not familiar with the ERAS programme and which applied a traditional care protocol, were contacted in order to enroll additional laparoscopic liver resection patients. These included the Academic Medical Centre (AMC), Amsterdam, the University Medical Centre Utrecht (UMCU), Utrecht, and the University Medical Centre Groningen (UMCG), Groningen.

The following recovery data were defined (and patients were considered functionally recovered when all criteria were met): normal or decreasing serum bilirubin; good pain control on oral analgesics only; absence of i.v. fluids and tolerance of solid food, and mobilization to preoperative level[4, 8]. Patients were considered fit for discharge, or functionally recovered, on the postoperative day when all four criteria were met. Patients were discharged if all four criteria were met and if they were willing to go home[4]. A discharge that occurred later than functional recovery was defined as a delayed discharge.

Primary outcome was total length of hospital stay (LOS), defined as the number of nights spent in hospital after surgery, including nights after readmission within 90 days of surgery. Secondary outcomes were functional recovery, complications, conversions, blood loss and length of operation. In addition, the following data were recorded: preoperative indication(s) for surgery; perioperative blood transfusions; use of nasogastric tubes; use of drains; restart of oral fluids; restart of oral solid food; histology of the resected liver specimen, and 
diameter of the lesions and resection margins.

Complications were defined according to the grading system devised by Clavien et al[12]. Grade I complications are generally non-life-threatening complications without lasting disability. Grade II complications are potentially life-threatening, but without residual disability. Grade III complications involve residual disability, including organ resections or persistence of life-threatening conditions. Grade IV complications refer to deaths as a result of complications.

All laparoscopic hepatic resections were performed by two consultant surgeons, one expert in hepatobiliary surgery and one with specific expertise in advanced laparoscopic surgery. Patients were placed in the 'French' position (supine position with legs apart) under general anaesthesia.The surgeonstoodbetween the legswith an assistant on each side[13]. Liver resections were defined according to anatomical planes (Couinaud's classification). An open introduction of the subumbilical port was used to produce the $\mathrm{CO} 2$ Pneumoperitoneum. In all cases, a 30-degree laparoscope was used and abdominal pressure was monitored and maintained at 12-14 $\mathrm{mmHg}$. Three to four extra trocars were placed in a semicircle, slightly higher and paramedian of the umbilical trocar, to facilitate the procedure[13]. Inspection of the liver was performed both visually and with laparoscopic ultrasound in order to confirm the number of lesions in relation to main anatomical structures. The falciform, left triangular ligament and lesser omentum were divided. Hepatic transection of the parenchyma and minor crossing vessels and biliary radicals was performed with a harmonic scalpel (Ultracision; Ethicon Endo-Surgery, Johnson \& Johnson, Piscataway, NJ, USA). A vascular stapler (EndoGIA; Covidien Surgical Devices, New Haven, CT, USA) was used to divide the portal pedicles and hepatic veins. Resected specimens were placed in a plastic bag (Endocatch; Covidien Surgical Devices) and removed through a separate, preferably suprapubic, incision. In cases where a laparotomy had been performed in the past, a small subumbilical incision was made for retrieval of the specimen.

\section{STATISTICAL ANALYSIS}

Continuous variables are expressed as median (range). Age, tumour diameter, hospital stay, functional recovery, delay in discharge, blood loss and duration of surgery were analysed using the Mann-Whitney U-test. Sex, American Society of Anesthesiologists (ASA) classification, indication for surgery, type of resection, liver pathology, complications, conversions, intake of fluid and solid food and the use of nasogastric tubes and drains were analysed using the chi-squared or Fisher's exact test as appropriate. A P-value $<0.05$ was considered statistically significant. Data were analysed using spss Version 13.0 for Windows (SPSS, Inc., Chicago, IL, USA). 


\section{RESULTS}

A total of 26 patients were treated by laparoscopic liver resection during the 5-year period in the four participating hospitals. Thirteen patients were treated by laparoscopic liver resections (left lateral sectionectomy [II, III] or segmentectomy [IV, V, VI] under the ERAS programme (group 1). All of these were operated at a single centre (MUMC). Thirteen patients were operated in traditional perioperative care settings in the four participating hospitals during the same period (group 2). Three laparoscopic procedures were performed at MUMC from 2003-2005 before the ERAS programme was instituted. Ten patients were operated laparoscopically in traditional perioperative care settings at the AMC $(n=4), \operatorname{UMCU}(n=2)$ and UMCG $(n=4)$. Demographics and clinical data are reported in Table 1.

\begin{tabular}{|c|c|c|c|}
\hline & $\begin{array}{l}\text { Group } 1 \\
\text { ERAS programme } \\
(n=13)\end{array}$ & $\begin{array}{l}\text { Group } 2 \\
\text { Traditional care } \\
(n=13)\end{array}$ & $\boldsymbol{P}$-value \\
\hline Age, years* & $55(34-82)$ & $45(26-70)$ & $0.104 \dagger$ \\
\hline ASA score I/II/III/IV & $3 / 9 / 1 / 0$ & $6 / 6 / 1 / 0$ & 0.688 \\
\hline Patient sex, M/F & $3 / 10$ & $2 / 11$ & 1.0 \\
\hline Indication for surgery: malignant/benign & $8 / 5$ & $3 / 10$ & 0.111 \\
\hline \multicolumn{4}{|l|}{ Types of liver resection } \\
\hline Left lateral sectionectomy & 9 & 9 & \\
\hline Monosegmentectomy (or metastasectomy) & 3 & 3 & 1.0 \\
\hline Multiple segmentectomy (>2 segments) & 1 & 1 & \\
\hline Liver pathology: malignant/benign & $5 / 8$ & $2 / 11$ & 0.160 \\
\hline Tumor size, $\mathrm{cm}^{*}$ & $4.1(1.0-10.5)$ & $6.0(1.4-8.0)$ & $0.765+$ \\
\hline
\end{tabular}

*Continuous variables are expressed as median (range) P-values by Fisher's exact test, except + Mann-Whitney U-test ERAS, Enhanced Recovery After Surgery; ASA, American Society of Anaesthesiologists; $M$, male; F, female

Median total LOS was reduced by 2 days in the ERAS group, but this difference did not attain statistical significance (Table 2). There were no readmissions in either group. There were two minor complications (grade I) in the ERAS group and two (grade I) in the traditional care group. In group 1, one patient had a urinary tract infection, which was treated by antibiotics, and another had constipation and remained in hospital longer. In group 2, one patient was treated for a hypokalaemia and another for atrial fibrillation. There were no deaths. The rate of conversions was similar in both groups (Table 2). In group 2 (traditional care), conversion occurred because of a preoperatively undetected lesion in segment IV in one patient and suboptimal visualization in another. The conversions in the ERAS group occurred because of the uncertain nature and size of the lesion and its proximity to the left liver vein in one patient and because of suboptimal visualization in another. Blood loss was significantly less 
in the ERAS group $(P<0.002)$. However, there was no difference in blood transfusions. Only one patient from group 2 (traditional care) received two units of packed cell while in hospital. No significant difference was observed in operation time. Oral fluid intake was resumed within 24 hours of surgery in almost all patients in the ERAS group, as well as in the traditional care group (median 1 day [range 0-2 days] vs. median 1 day [range 0-6 days], respectively; $\mathrm{P}=0.861$ ). The median time to successful resumption of normal diet was 1 day (range 1-2 days) in the ERAS group, compared with 2 days (range 1-6 days) in the controls ( $P=0.223$ ). Nasogastric decompression tubes were not used after surgery in the ERAS group. In the control group, nasogastric tubes were in place after surgery in five of 13 patients for $1-5$ days $(P=0.257)$. This included two reinsertions because of delayed gastrointestinal function on day 1. In the ERAS group, no drains were used after surgery. In the control group, six patients received a drain, which was removed $2-4$ days after surgery $(P=0.015)$.

In both groups, functional recovery was achieved a median of 2 days earlier than actual discharge. In the ERAS group, patients met the discharge criteria on median postoperative day 3 (range 1-7 days), compared with the traditional care group, which met the discharge criteria on median postoperative day 5 (range 2-8 days). The difference between the groups in functional recovery proved to be statistically significant $(P<0.044)$, as did the difference in delay in discharge $(\mathrm{P}<0.032)$.

In group 1, resected specimens included two hepatocellular adenomas, two focal nodular hyperplasias (FNHs), an angiomyolipoma, a haemangioma, a presumed metastasis which proved to be an inflammatory pseudotumour after chemotherapy, an intrahepatic accessory gallbladder with inflammation, a hepatocellular carcinoma and four metastases of colorectal origin. Resection margins were free of disease in 12 cases; one resected adenoma extended to the resection margin. In group 2, resected specimens included three adenomas, six FNHs and two haemangiomas and, in two patients, three metastases of colorectal origin. Resection margins were free of disease in eight patients, were not reported (benign tumour) in four and involved a haemangioma in the resection surface in one. No late complications were recorded in group 1 during the follow-up period (median 3 months, range 1-23 months). In the follow-up period (median 6 months, range 1-27 months) in group 2,one patient developed colorectal metastases in the lungs and liver (segments VI and VII) and simultaneous acute myeloid leukaemia. No palliative chemotherapy was given to this patient because of his clinical condition and he died 12 months postoperatively. 


\begin{tabular}{|c|c|c|c|}
\hline & $\begin{array}{l}\text { Group } 1 \\
\text { ERAS programme } \\
(n=13)\end{array}$ & $\begin{array}{l}\text { Group } 2 \\
\text { Traditional care } \\
(n=13)\end{array}$ & $\boldsymbol{P}$-value \\
\hline \multicolumn{4}{|l|}{ Primary Outcome } \\
\hline Total LOS, days* & $5.0(3-10)$ & $7.0(3-12)$ & $0.305 t$ \\
\hline \multicolumn{4}{|l|}{ Secondary outcomes } \\
\hline Functional recovery, days* & $3(1-7)$ & $5(2-8)$ & $0.044 \dagger$ \\
\hline Complications, $\mathrm{n}$ (grade) & $2(\mathrm{I})$ & $2(\mathrm{I})$ & 1.0 \\
\hline Conversions, $\mathrm{n}$ & 2 & 2 & 1.0 \\
\hline Blood loss, mi & $50(50-200)$ & $250(50-800)$ & $0.002 \dagger$ \\
\hline Operation time, $\min ^{*}$ & $118(85-192)$ & $180(51-340)$ & $0.293 t$ \\
\hline
\end{tabular}

*Continuous variables are expressed as median (range) P-values by Fisher's exact test, except TMann-Whitney U-test ERAS, Enhanced Recovery After Surgery; LOS, length of stay

Table 2 Primary and secondary outcomes. No readmissions were recorded in either group 


\section{DISCUSSION}

This study investigated the added value of an enhanced recovery programme in laparoscopic liver surgery. To the best of our knowledge, this represents the first such study to be conducted (although it is only a small pilot study). The results suggest that initiation of a multimodal enhanced recovery programme in laparoscopic liver surgery is feasible. It also shows that it is safe to implement an evidence-based enhanced recovery programme, as there were only two minor complications in the ERAS group and two conversions in each group. The difference in median LOS of 2 days did not attain significance, but this probably reflects the small number of patients in this study. Importantly, the current study provides evidence that an enhanced recovery programme is associated with expedited functional recovery. Patients in the laparoscopic ERAS group fulfilled discharge criteria 2 days earlier than control patients.

Patients in the enhanced recovery group had less blood loss. This may have resulted from the implementation of the ERAS protocol, which prescribes a low CVP and the avoidance of excessive perioperative fluids. However, although this outcome is significant, it remains unclear whether it resulted solely from the implementation of ERAS. This difference in blood loss may also have been clouded by the learning curve effect because the patients operated in group 2 (traditional care) were the first patients to be treated laparoscopically in the participating centres. Most authors recommend that laparoscopic procedures on liver resections of solid tumours should preferably begin with the left lateral segments or anterolateral small lesions of the right side[13,14]. Tumours of the left lateral side, particularly benign tumours, constitute only a small fraction of the minor resections in most units. This is probably one of the reasons for the small number of patients in this pilot study. It was also considered unethical to prospectively compare effects in an intervention (ERAS) group with effects in a control group receiving traditional care in Maastricht because the ERAS protocol includes several evidence-based care elements aimed at reducing surgical stress and postoperative catabolism[6-8].

Studies on colorectal laparoscopy and fast-track programmes have shown conflicting results regarding the additional outcomes of minimally invasive surgery performed within a perioperative ERAS programme. In a single-centre study, the laparoscopic approach seemed to improve the outcome in terms of LOS, whereas other studies, including a randomized controlled trial, showed no additional effect of laparoscopy on LOS[15-17] . Therefore, it has been suggested that there is a need for further randomized trials to evaluate the additional effects of laparoscopy within fast-track recovery programmes in colorectal surgery $[5,15,16]$. At present, there is only limited evidence available about the effects on LOS of a multimodal, fast-track setting in liver surgery[4]. In the current study, a difference between the date of functional recovery of patients undergoing liver resection and the day of actual discharge 
was observed, as we previously reported in a colonic ERAS programme[11]. A significant difference of a median of 2 days in time to full functional recovery was observed between the ERAS-treated group and the traditional care group. It is remarkable that, despite the preoperative counselling in the ERAS programme, a difference between functional recovery and actual day of discharge was still observed. This implies that the difference in LOS may be even more pronounced if patients do actually go home as soon as they are functionally recovered according to predefined criteria[ $[8,11]$. Delay in discharge and presence of complications were associated with increased LOS. For future studies, functional recovery may be a better criterion with which to investigate differences in outcome of surgical treatments. Establishing the effect of ERAS programmes on outcomes in laparoscopic liver surgery in a more structured manner is a challenge yet to be met. 


\section{REFERENCES}

1. Gagner, M., et al., Laparoscopic adrenalectomy: lessons learned from 100 consecutive procedures. Ann Surg, 1997. 226(3): p. 238-46; discussion 246-7.

2. Trondsen, E., et al., Laparoscopic and open cholecystectomy. A prospective, randomized study. Eur J Surg, 1993. 159(4): p. 217-21.

3. Simillis, C., et al., Laparoscopic versus open hepatic resections for benign and malignant neoplasms--a meta-analysis. Surgery, 2007. 141(2): p. 203-211.

4. van Dam, R.M., et al., Initial experience with a multimodal enhanced recovery programme in patients undergoing liver resection. Br J Surg, 2008. 95(8): p. 969-75.

5. Wind, J., et al., Systematic review of enhanced recovery programmes in colonic surgery. Br J Surg, 2006. 93(7): $p$. 800-9.

6. Fearon, K.C., et al., Enhanced recovery after surgery: a consensus review of clinical care for patients undergoing colonic resection. Clin Nutr, 2005. 24(3): p. 466-77.

7. Kehlet, $\mathrm{H}$., Manipulation of the metabolic response in clinical practice. World J Surg, 2000. 24(6): p. 690-5.

8. Maessen, J., et al., A protocol is not enough to implement an enhanced recovery programme for colorectal resection. Br J Surg, 2007. 94(2): p. 224-31.

9. Podore, P.C. and E.B. Throop, Infrarenal aortic surgery with a 3-day hospital stay: A report on success with a clinical pathway. J Vasc Surg, 1999. 29(5): p. 787-92.

10. Trondsen, E., et al., Day-case laparoscopic fundoplication for gastro-oesophageal reflux disease. Br J Surg, 2000. 87(12): p. 1708-11

11. Maessen, J.M., et al., Length of stay: an inappropriate readout of the success of enhanced recovery programs. World J Surg, 2008. 32(6): p. 971-5.

12. Clavien, P.A., J.R. Sanabria, and S.M. Strasberg, Proposed classification of complications of surgery with examples of utility in cholecystectomy. Surgery, 1992. 111(5): p. 518-26.

13. Cherqui, D., et al., Laparoscopic liver resections: a feasibility study in 30 patients. Ann Surg, 2000. 232(6): p. $753-62$.

14. Gigot, J.F., et al., Laparoscopic liver resection for malignant liver tumors: preliminary results of a multicenter European study. Ann Surg, 2002. 236(1): p. 90-7.

15. Basse, L., et al., Functional recovery after open versus laparoscopic colonic resection: a randomized, blinded study. Ann Surg, 2005. 241(3): p. 416-23.

16. MacKay, G., et al., Laparoscopic colonic resection in fast-track patients does not enhance short-term recovery after elective surgery. Colorectal Dis, 2007. 9(4): p. 36872.

17. Raue, W., et al., 'Fast-track' multimodal rehabilitation program improves outcome after laparoscopic sigmoidectomy: a controlled prospective evaluation. Surg Endosc, 2004. 18(10): p. 1463-8. 


\section{ORANGETRIAL}
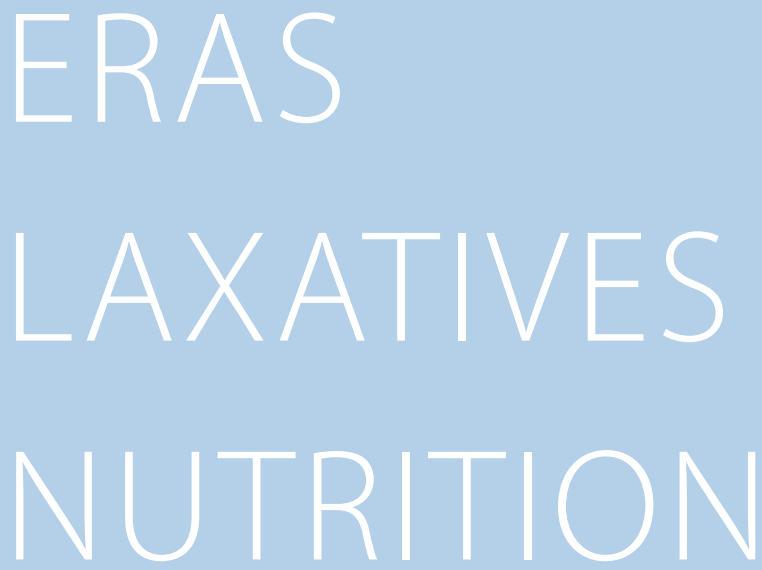

\section{PUBLISHED AS}

Randomized clinical trial of laxatives and oral nutritional supplements within an enhanced recovery after surgery protocol following liver resection

P. O. Hendry, R.M. van Dam, S.F. F.W. Bukkems, D.W. McKeown, R.W. Parks, T. Preston, C.H.C. Dejong, O. J. Garden and K. C.H. Fearon, on behalf of the Enhanced Recovery After Surgery (ERAS) Group

British Journal of Surgery 2010; 97: 1198-1206

DOI: dx.doi.org/10.1002/bjs.7120 


\section{ABSTRACT}

\section{BACKGROUND}

Routine laxatives may expedite gastrointestinal recovery and early tolerance of food within an enhanced recovery after surgery (ERAS) programme. Combined with carbohydrate loading and oral nutritional supplements (ONS), it may further enhance recovery of gastrointestinal function and promote earlier overall recovery.

\section{METHODS}

Seventy-four patients undergoing liver resection were randomized in a two-by-two factorial design to receive either postoperative magnesium hydroxide as a laxative, preoperative carbohydrate loading and postoperative ONS, their combination or a control group. Patients were managed within an ERAS programme of care. The primary outcome measure was time to first passage of stool. Secondary outcome measures were gastric emptying, postoperative oral calorie intake, time to functional recovery and length of hospital stay.

Sixty-eight patients completed the trial. The laxative group had a significantly reduced time to passage of stool: median (interquartile range) $4(3-5)$ versus $5(4-6)$ days $(P=0.034)$. The ONS group showed a trend towards a shorter time to passage of stool $(P=0.076)$ but there was no evidence of interaction in patients randomized to the combination regimen. Median length of hospital stay was 6 (4-7) days. There were no differences in secondary outcomes between groups.

\section{CONCLUSION}

Within an ERAS protocol for patients undergoing liver resection, routine postoperative laxatives result in an earlier first passage of stool but the overall rate of recovery is unaltered. Registration number: NCT00538954 (http://www.clinicaltrials.gov). 


\section{INTRODUCTION}

If left unchecked, the metabolic response after abdominal surgery can result in prolonged recovery, increased morbidity and a longer hospital stay[1, 2]. Enhanced recovery after surgery (ERAS) programmes combine interventions that target the elements that delay recovery: pain, gastro-intestinal dysfunction and immobility[3]. Such protocols are safe, effective, accelerate recovery and reduce hospital stay following liver resection[4], aortic aneurysm repair[5], oesophagectomy[6] and colorectal resection[7-9].

Evidence for the efficacy of individual protocol elements within an ERAS programme is often extrapolated from traditional care[3]. Morbidity and hospital stay are relatively predictable after liver resection, which provides a suitable model for testing the individual elements within the ERAS protocol[10-12].

Reduced gastrointestinal function is a major factor that limits the success of enhanced recovery. Current protocols employ a multimodal package including continuous thoracic epidural anaesthesia, enforced mobilization, avoidance of excessive saline and early feeding which, in traditional perioperative care, can reduce postoperative ileus[13-15] or promote earlier passage of stool[16]. Administration of magnesium hydroxide has been described in an unblinded, non-randomized study and employed in ERAS programmes[17]; however, despite widespread use of laxatives there is limited evidence to justify their routine postoperative use[7, 17]. Preoperative carbohydrate loading may also promote earlier return of gastrointestinal function[18]. Within an ERAS protocol, carbohydrate loading and early oral nutritional supplements (ONS) may interact with laxatives to enhance gastrointestinal recovery further.

Early gastrointestinal recovery allows early oral intake, discontinuation of intravenous fluids and accelerated recovery. Early food intake may attenuate postoperative catabolism. Oral carbohydrate loading before surgery can also reduce this catabolic response and abate insulin resistance[19, 20]. By improving the response to anabolic stimuli in the postoperative period, metabolic preconditioning may promote greater benefit from early nutritional intake. The perioperative administration of ONS has been shown to improve nutritional status and reduce minor complications within traditional perioperative care[21, 22]. It is not known whether the provision of combined metabolic conditioning/early oral nutritional support along with early recovery of gastrointestinal function achieved with magnesium hydroxide can promote more rapid overall recovery within an ERAS protocol.

The aim of the Optimized Recovery with Accelerated Nutrition and Gastrointestinal Enhancement (ORANGE) study was to determine, within an ERAS protocol designed for hepatic resection, whether postoperative bowel stimulation with magnesium hydroxide and/ 
or metabolic conditioning/ONS are effective in promoting early return of gastrointestinal function and overall postoperative recovery.

\section{METHODS}

This prospective randomized controlled trial with a two by-two factorial design was conducted with the approval of Lothian Research Ethics Committee and the Ethics Committee of Maastricht University Medical Centre. Patients gave their written informed consent to participate. All were between the ages of 18 and 80 years, and scheduled to undergo hepatic resection for benign or malignant conditions at the Liver Unit, Royal Infirmary, Edinburgh, UK, or Maastricht University Medical Centre, The Netherlands, between July 2006 and June 2008. Patients with a body mass index of less than 18 or greater than $30 \mathrm{~kg} / \mathrm{m} 2$, preexisting conditions limiting mobility, underlying cirrhotic liver disease, a history of liver resection, and those in whom bile duct excision and central or extended hepatectomy was planned before randomization, were excluded. Recruitment took place during preoperative assessment at least 1 week before admission, allowing a period of reflection before obtaining consent. Patients received information packs and counselling related to the ERAS programme, highlighting early mobilization and early oral intake.

\section{ORANGETRIAL PROTOCOL}

Patients were randomized to one of four groups (control, laxatives, ONS and laxatives + ONS). Randomization was performed using sealed opaque envelopes created in advance through block randomization with a random numbers table. Owing to the nature of the study interventions (nutritional supplements available in a variety of flavours to encourage compliance and magnesium hydroxide suspension produced by the hospital pharmacy), a suitable placebo was not feasible and so the type of treatment could not be masked. Patients randomized to laxatives received $1 \mathrm{~g}$ magnesium hydroxide orally twice daily from the day of surgery until discharge. Patients randomized to ONS received $800 \mathrm{ml}$ oral carbohydrate loading drink (Nutricia Preop; Nutricia Clinical Care, Trowbridge, UK.) at 22.00 hours the night before surgery and $400 \mathrm{ml}$ at 06.00 hours on the morning of surgery. In addition, they received ONS (two cartons per day comprising $400 \mathrm{ml}, 600 \mathrm{kcal}, 24 \mathrm{~g}$ protein, Nutricia Fortisip; Nutricia Clinical Care) from the day of surgery until day 30. Patients randomized to the laxatives + ONS group received the combined regimen.

A standardized perioperative and anaesthetic regimen, based on a previous ERAS protocol, was followed[3].This multimodal, enhanced recovery programme was modified to cover all aspects of liver resection[4]. Notably, liver tissue sealant (TachoSil; Nycomed, Zurich, Switzerland) was used to minimize bile leakage and haematoma formation, routine perihepatic drainage was avoided and paracetamol was used at the discretion of the surgeon in cases of 
extended resection. Patients were normally scheduled for surgery in the morning and were nursed in a high-dependency unit. Details of the ERAS liver protocol are shown in Table 1.

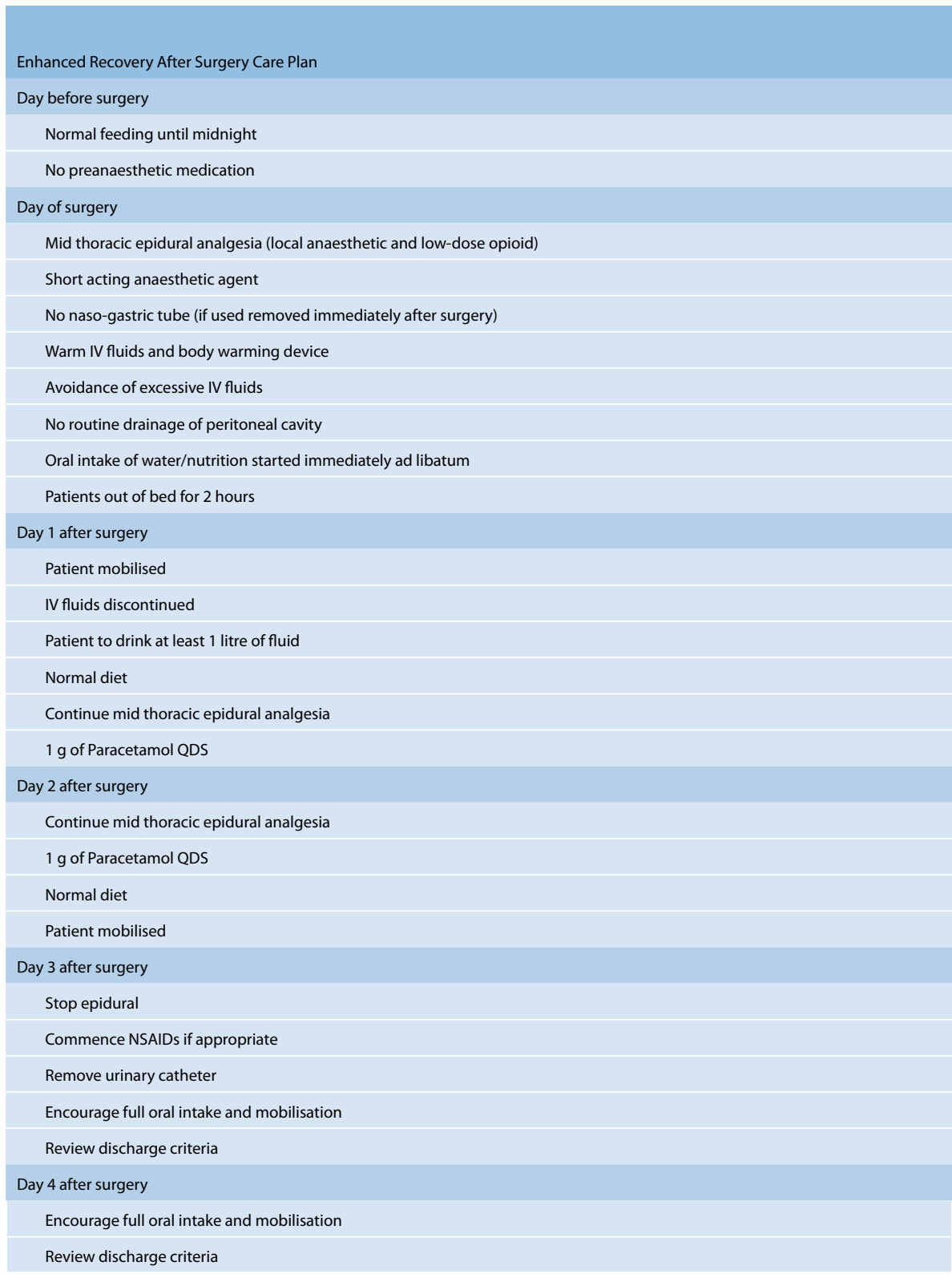


Seven surgeons performed all resections between the two centres. The extent of resection was classified according to the Brisbane 2000 Terminology[23]. Subcostal incisions were used and the transection plane determined by preoperative imaging and use of intraoperative ultrasonography. Resection was performed using a Cavitron Ultrasonic Surgical Aspirator (CUSA; Valleylab, Boulder, Colorado, USA) and argon beam coagulation. The raw surface of the liver remnant was subjected to argon beam coagulation and sealed with TachoSil. Abdominal drains were not placed routinely and the abdomen was closed with a standard running suture.

Study data were documented prospectively during the hospital stay and 30 days after surgery at an outpatient visit. Return of gastrointestinal function was determined by the first passage of stool after surgery. Patients were assessed for return of gastrointestinal function (passage of flatus/stool) on a daily basis. Secondary outcomes were: gastric emptying rate on day 3 after operation as determined by stable isotope breath test, postoperative oral nutritional intake on days 1-3 after surgery, functional recovery and length of stay.

\begin{tabular}{|c|c|c|c|c|c|c|c|}
\hline & Overall & $\begin{array}{l}\text { No. } \\
\text { Laxation }\end{array}$ & Laxation & $\mathrm{p}$ & No ONS & ONS & $p$ \\
\hline & 68 & 34 & 34 & & 38 & 30 & \\
\hline$M: F$ & $30: 38$ & $21: 13$ & $17: 17$ & $0,464 \ddagger$ & $20: 18$ & $18: 12$ & $0.626 \ddagger$ \\
\hline Age (years)* & $\begin{array}{c}62 \\
(53,69)\end{array}$ & $\begin{array}{c}62 \\
(51,70)\end{array}$ & $\begin{array}{c}61,5 \\
(55,69)\end{array}$ & $0.722 \dagger$ & $\begin{array}{c}64.59 \\
(51,70)\end{array}$ & $\begin{array}{c}61 \\
(52,63)\end{array}$ & $0.243+$ \\
\hline \multicolumn{8}{|l|}{ ASA } \\
\hline 1 & $15(22.1)$ & $7(20.6)$ & $8(23.5)$ & & $9(23.7)$ & $6(20.0)$ & \\
\hline 2 & $43(63.2)$ & $20(58.8)$ & $23(67.6)$ & & $23(60.5)$ & $20(66.7)$ & \\
\hline 3 & $10(14.7)$ & $7(20.6)$ & $3(8.8)$ & $0.391 \neq$ & $6(15.8)$ & $4(13.3)$ & $0.973 \neq$ \\
\hline 4 & $0(0.0)$ & $0(0)$ & $0(0)$ & & $0(0)$ & $0(0)$ & \\
\hline $\mathrm{BMI}^{*}$ & $\begin{array}{c}22 \\
(22.5,28)\end{array}$ & $\begin{array}{c}25.25 \\
(22.6,30.5)\end{array}$ & $\begin{array}{c}24.95 \\
(22.3,28)\end{array}$ & $0.394 \dagger$ & $\begin{array}{c}25.05 \\
(22.2,28.0)\end{array}$ & $\begin{array}{c}25.1 \\
(22.6,28.9)\end{array}$ & $0.882 \dagger$ \\
\hline \multicolumn{8}{|l|}{ Pathology } \\
\hline $\begin{array}{l}\text { Colorectal Liver } \\
\text { Metastases }\end{array}$ & $56(82.4)$ & $26(76.5)$ & $30(88.2)$ & & $31(81.6)$ & $25(83.3)$ & \\
\hline $\begin{array}{c}\text { Other metastatic } \\
\text { disease }\end{array}$ & $6(8.8)$ & $4(11.8)$ & $2(5.9)$ & & $4(10.5)$ & $2(6.7)$ & \\
\hline Benign disease & $6(8.8)$ & $4(11.8)$ & $2(5.9)$ & $0.445 \ddagger$ & $3(7.9)$ & $3(10.0)$ & $0.829 \neq$ \\
\hline Malignant Disease (n) & $63(92.6)$ & $31(91.2)$ & $32(94.1)$ & $1 \neq$ & $35(92.1)$ & $28(93.3)$ & $1 \neq$ \\
\hline $\begin{array}{l}\text { Neoadjuvent therapy } \\
\text { (n) }\end{array}$ & $22(32.4)$ & $9(26.5)$ & $13(38.2)$ & $0.127 \ddagger$ & $13(34.2)$ & $9(30.0)$ & $0.797 \neq$ \\
\hline
\end{tabular}




\section{STABLE ISOTOPE GASTRIC EMPTYING BREATHTEST}

On the third day after surgery and following an overnight fast, some patients in one centre underwent assessment of gastric emptying rate by means of a non-invasive stable isotope breath test. This employed a 200-ml liquid test meal (Nutricia Fortisip) containing a stable isotope tracer (75 mg sodium 1-[13C]-Acetate)[24]. Breath samples were collected before and at predetermined intervals following consumption of the test meal. During the test period, activity was restricted to allow control and estimation of the rate of elimination of carbon dioxide[25]. The [13C]-Carbon dioxide enrichment of breath samples (13C: 12C-ratio) was measured by automated continuous-flow isotope ratio mass spectrometry[26]. Raw data were subjected to curve fitting to derive the half-time ( $T 1 / 2)$ of [13C]-Carbon dioxide appearance in breath[27]. The $T 1 / 2$ for gastric emptying was estimated using a 'self-correcting' model that accounts for the delayed expiration of [13C]-Carbon dioxide owing to pooling within the body's bicarbonate system[28].

\section{ORAL NUTRITIONAL INTAKE}

A senior dietician, in cooperation with the patient and ward nursing staff, recorded daily postoperative oral intake on a standard diet sheet. This was converted to energy intake (in kilocalories) and the recommended nutritional intake (RNI) was estimated using CompEat Pro for Windows (Nutrition Systems, Banbury, UK). The percentage RNI achieved on the first 3 days after surgery for each subject was used in comparisons between study groups.

\section{FUNCTIONAL RECOVERY CRITERIA}

Functional recovery was defined as adequate pain control requiring only oral analgesia, adequate oral intake with no intravenous fluid requirement, independent mobility sufficient to perform activities of daily living, and blood results (liver function tests and inflammatory markers) returning towards normal ranges[4]. Criteria were assessed on a daily basis. An experienced clinician determined readiness for hospital discharge. Time to achieve functional recovery, initial length of stay (defined as number of nights in hospital after surgery, excluding readmissions) and all adverse events up to 30 days were recorded. Morbidity was documented prospectively according to criteria described previously[29, 30]. Other data recorded for each patient included mortality, readmissions and use of preoperative chemotherapy.

\section{STATISTICAL ANALYSIS}

Retrospective data (P. O. Hendry and K. C. H. Fearon, unpublished results) indicated that the mean(s.d.) time to passage of stool was $5 \cdot 3(1 \cdot 3)$ days. This study was powered ( $a=0.05, \beta$ $=0 \cdot 80$ ) to detect a 20 per cent difference in time to passage of stool, the primary outcome. Based on this calculation, 14 patients were required in each of the four groups (56 patients overall). The secondary endpoints were of an exploratory nature. Continuous data were expressed as median (interquartile range). For comparison between study groups, data were analysed using Mann-Whitney $U$ test, $X 2$ test or Fisher's exact test, as appropriate. $P<0.050$ 
was considered statistically significant. SPSS®version 17.0 for Mac (SPSS, Chicago, Illinois, USA) was used for data analysis.

\section{RESULTS}

Between July 2006 and June 2008, 74 patients were recruited and randomized, 66 in Edinburgh and eight in Maastricht. Six patients did not undergo hepatic resection and were excluded. The remaining 68 patients were managed within the ORANGE protocol (Figure 1).

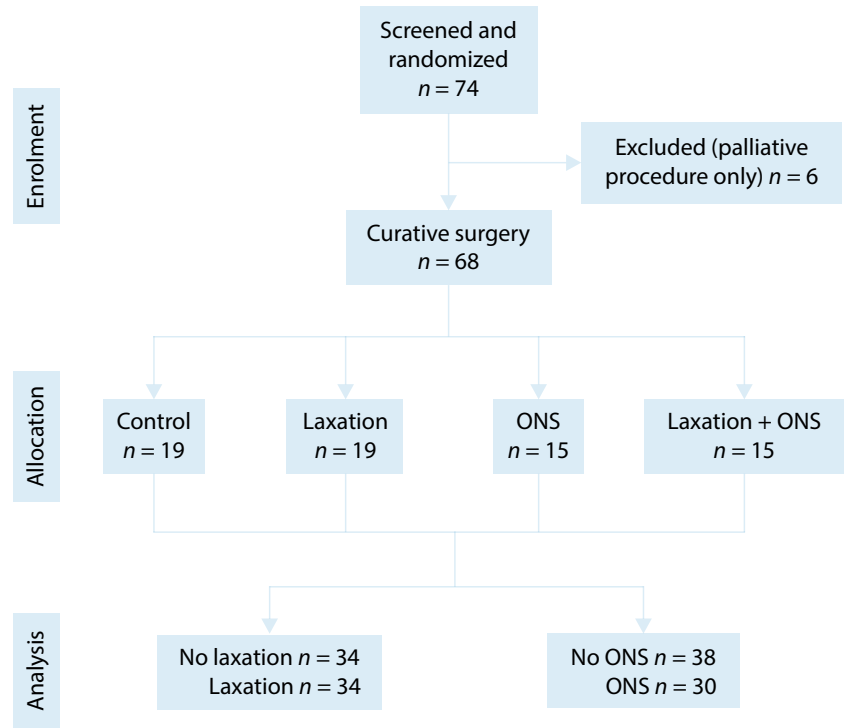

Fig. 1 CONSORT diagram for the trial. ONS, oral nutritional supplements

Demographic data for the four groups are shown in Table 2. Overall the male to female ratio was $4: 3$ and median age was 62 (53-69) years. Sixty-two patients (91 per cent) were treated for malignant disease, and there were 53 major and 15 minor resections (Table 3 ). There were no significant differences across the study groups in sex, body mass index, American Society of Anesthesiologists grade, pathology, malignancy, use of neoadjuvant therapy or extent of liver resection.

\begin{tabular}{|c|c|c|c|c|c|c|c|}
\hline Operation & $\begin{array}{l}\text { Overall } \\
n=68\end{array}$ & $\begin{array}{c}\text { No Laxa- } \\
\text { tion } \\
34\end{array}$ & $\begin{array}{c}\text { Laxation } \\
34\end{array}$ & $p$ & $\begin{array}{c}\text { No ONS } \\
38\end{array}$ & $\begin{array}{l}\text { ONS } \\
30\end{array}$ & $p$ \\
\hline $\begin{array}{l}\text { Major (trisectionectomy, central hepatectomy, } \\
\text { hemihepatectomy) }\end{array}$ & 53 & $25(73.5)$ & $28(82.4)$ & & $31(81.6)$ & $22(73.3)$ & \\
\hline $\begin{array}{l}\text { Minor (sectionectomy, segmentectomy, } \\
\text { metastasectomy) }\end{array}$ & 15 & $9(26.5)$ & $6(17.6)$ & $0.560 \S$ & $7(18.4)$ & $8(26.7)$ & $0.557 \S$ \\
\hline
\end{tabular}


Time to functional recovery and initial length of hospital stay were 4 (3-5) and 6 (4-7) days respectively, and were not significantly different between groups. The postoperative morbidity rate was 25 percent overall, and did not differ significantly between the groups. Two patients required reoperation owing to postoperative haemorrhage. Five patients required readmission to hospital (intra-abdominal collection, 2; severe constipation, 1; bile leak, 1; pneumonia, 1). There were two deaths from myocardial infarction within 30 days of surgery (Table 4).

\begin{tabular}{|c|c|c|c|c|c|c|c|}
\hline & $\begin{array}{l}\text { Overall } \\
n=68\end{array}$ & $\begin{array}{c}\text { No Laxation } \\
34\end{array}$ & $\begin{array}{l}\text { Laxation } \\
\quad 34\end{array}$ & $\mathrm{p}$ & $\begin{array}{l}\text { No ONS } \\
38\end{array}$ & $\begin{array}{l}\text { ONS } \\
30\end{array}$ & $\mathrm{p}$ \\
\hline Time to first drink & $0(0,0)$ & $0(0,0)$ & $0(0,0)$ & $1.000+$ & $0(0,0)$ & $0(0,0)$ & $0.808+$ \\
\hline Time to first food & $1(1,1)$ & $1(0,1)$ & $1(0,1)$ & $0.218+$ & $1(0,1)$ & $1(0,1)$ & $0.499+$ \\
\hline Time to first flatus & $3(2,4)$ & $3(2,4)$ & $2.5(2,3.5)$ & $0.108 t$ & $3(2,5)$ & $3(2,4)$ & $0.262 \dagger$ \\
\hline Time to first stool & $5(4,6)$ & $5(4,6)$ & $4(3,5)$ & $0.034 t$ & $5(3.5,7)$ & $4(3,5)$ & $0.076+$ \\
\hline $\begin{array}{l}\text { Time to functional } \\
\text { recovery }\end{array}$ & $4(3,5)$ & $4(3,4.5)$ & $4(3,5)$ & $0.242 \dagger$ & $4(3,5)$ & $4(3,4)$ & $0.110 \dagger$ \\
\hline Initial hospital stay & $6(4,7)$ & $5(4,6)$ & $6(5,8)$ & $0.081 \dagger$ & $6(5,7)$ & $5(4,7)$ & $0.367 \dagger$ \\
\hline Readmission* & $5(7.4)$ & $3(88.2)$ & $2(5.9)$ & $1.000 \S$ & $3(7.9)$ & $2(6.7)$ & $1.000 \S$ \\
\hline Reoperation* & $2(2.9)$ & 1(2.9) & $1(2.9)$ & $1.000 \S$ & $2(5.3)$ & $0(0.0)$ & $0.504 \S$ \\
\hline 30-day morbidity* & $17(25)$ & $8(23.5)$ & $9(26.5)$ & $1.000 \S$ & $11(28.9)$ & $6(20.0)$ & $0.574 \S$ \\
\hline 30-day mortality* & $2(2.9)$ & 2(5.9) & $0(0.0)$ & $0.494 \S$ & $2(5.3)$ & $0(0.0)$ & $0.500 \S$ \\
\hline
\end{tabular}

Table 4 Outcome data

Discharge from hospital was delayed in some patients for reasons outlined in Table 5. Oral fluid intake was resumed on the day of surgery in 94 percent of patients. Reintroduction of diet was achieved on day 1 in 37 percent and by day 2 in 91 percent. Intravenous fluids were discontinued on day 1 in 51 percent of patients and by day 2 in 78 percent. Mid-thoracic epidural anaesthesia was commenced before surgery in 99 percent of patients and continued for at least $48 \mathrm{~h}$ after operation in 93 percent; four epidurals failed on day 1 .

\begin{tabular}{|l|c|}
\hline & $\begin{array}{c}\text { No. of } \\
\text { patients }\end{array}$ \\
\hline Caution owing to earlier complication or extensive surgery & 5 \\
\hline Patient not confident to be discharged & 4 \\
\hline Transport or social problems & 2 \\
\hline Patient boarded to another ward before discharge & 1 \\
\hline Patient drowsy owing to analgesia & 1 \\
\hline
\end{tabular}


Drains were used in nine patients (13 percent). Nineteen patients ( 28 percent) achieved mobilization on the day of surgery. Sixteen patients ( 24 percent) had a urinary catheter in place beyond discontinuation of epidural analgesia and 32 (47 percent) received non-protocol intravenous fluids in the perioperative period. Overall, the median time to passage of stool was 5 (4-6) days. Patients randomized to ONS showed a trend towards a shorter time to passage of stool: $4(3-5)$ versus $5(4-7)$ days $(P=0.076)$. Those randomized to laxatives had a significantly reduced time to passage of stool: $4(3-5)$ versus $5(4-6)$ days $(P=0.034)$. Patients randomized to the combination regimen also had a significantly reduced time to passage of stool: 3 (3-4) versus $6(4-7)$ days $(P=0.013)$. There was no evidence of interaction between laxatives and ONS (Figure 2).

Thirty-two of 56 patients asked to participate in the gastric emptying protocol completed it (7 control, 10 laxative, 8 ONS, 7 laxative + ONS). Median postoperative gastric emptying (T1/2) was $0.74(0.46-1.41)$ h, with no significant difference between study groups. Five patients had significantly delayed gastric emptying ( $T 1 / 2$ more than the 90th percentile of 1.69 h). In comparison with the remaining 27 patients who underwent measurement of gastric emptying rate, this group had a significantly prolonged hospital stay: 10 (7-11) versus 6 (5-7) days $(P=0.019)$. Oral intake of at least 50 per cent of recommended nutritional requirement was achieved at a median of $1(1-2)$ days after surgery for the whole group. This was not significantly different between groups. Total calorie intake (including ONS) on days 1-3 after surgery for those randomized to ONS was not significantly different from that in patients who did not receive ONS (Figure 3). The median percentage change in bodyweight from preoperative level for all subjects was $+1.82(-1.41$ to 5.82$)$ and $-2.01(-4.65$ to -0.34$)$ percent on days 5 and 30 respectively after surgery; there were no significant differences between groups $(P=0.166$ and $P=0.200$ respectively). 


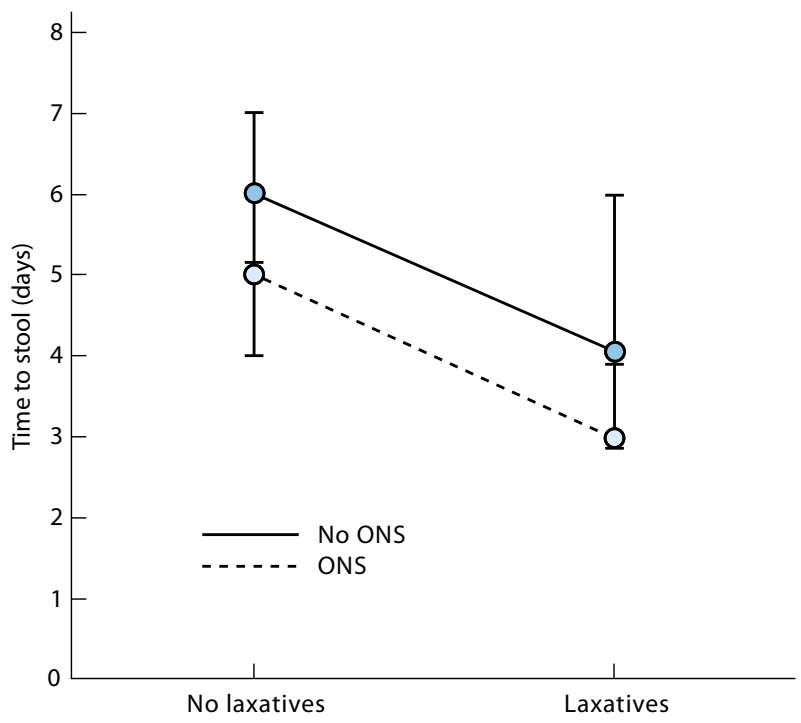

Fig. 2 Interaction between laxatives and oral nutritional supplements (ONS). Values are median with interquartile range

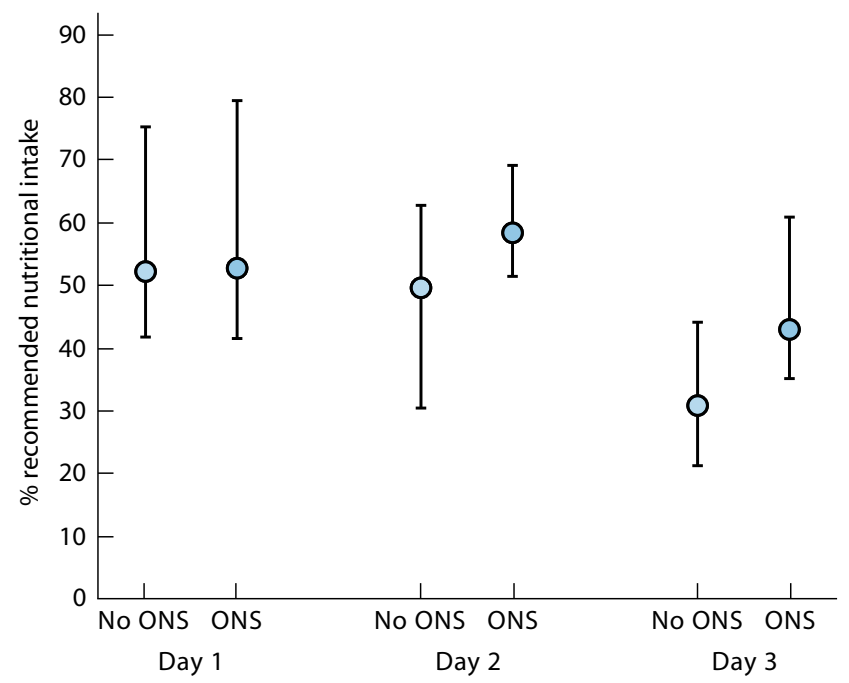

Values are median with interquartile range. ONS, oral nutritional supplements 


\section{DISCUSSION}

In this study, laxatives significantly decreased the time to passage of stool after liver surgery. Those randomized to the combination regimen also had a significantly reduced time to passage of stool, but with no evidence of interaction between laxatives and ONS. There were no differences in secondary outcomes between groups. These results are in keeping with previous findings[31, 32].

Previous studies suggested that early restoration of food intake results in a more rapid return of gut function in the postoperative period[16]. In the present study, calorie intake (food plus ONS) of the group randomized to ONS was no different from that of patients who did not receive ONS, suggesting that ONS in the early postoperative phase suppressed normal food intake to the point at which there was no net benefit. The lack of interaction between laxatives and ONS could therefore be explained on the basis that ONS did not alter overall calorie intake. This would also explain the lack of effect of ONS on the secondary outcomes. Patients following the ERAS protocol recommenced oral fluid on the day of surgery, started eating the following day, were mobile by the third day and achieved discharge criteria on the fourth day. Median hospital stay was 6 days, which is significantly shorter than the 8-14 days reported in other centres following traditional perioperative care.[11, $12,33,34]$. This study provides further evidence to support the use of an ERAS pathway following liver resection to accelerate postoperative recovery and shorten length of stay. It remains unclear which components of reduced stay result from the introduction of a formal care pathway rather than the enhanced recovery elements of that pathway. In the present study, functional recovery was achieved a median of 4 days after surgery, a median of 2 days before discharge from hospital. It may be possible to achieve a shorter overall hospital stay by further tightening the care pathway elements of the protocol [35].

In this study, the overall morbidity rate was 25 percent, with a readmission rate of 7 percent and mortality rate of 3 percent. Morbidity rates were lower and mortality rates in keeping with those published from other countries following traditional care (38-45 and 2.7-3.1 percent respectively).[11,33,36]. All patients received preoperative counselling and there was a high level of compliance with most elements of the protocol. Ninety-nine percent of patients received preoperative epidural anaesthesia. Intraoperative drainage was used only in patients considered to have a higher risk of bile leakage (13 percent), in keeping with current evidence [37, 38]. In the early postoperative period, adherence to the protocol was lower than in the preoperative and intraoperative phases, as in previous studies[35, 39].

Intravenous fluids were discontinued on the day after surgery in only half of the patients. Saline ( 0.9 percent) was often prescribed by out-of-hours medical staff, less familiar with the study protocol. Only 28 percent of patients were mobilized on the day of surgery; usual reasons for failure to achieve this were epidural-related hypotension or late return to the 
high-dependency unit. Future ERAS protocols may overcome the issue of late return to the ward by initiating mobilization in the recovery room. Postoperative variables are markers of both protocol compliance and recovery; patients achieving these protocol goals are those likely to achieve a faster recovery.

There were no significant differences between study groups in the rate of gastric emptying. The gastric emptying protocol was available for 56 patients in the study, but it was impractical for 15 and refused by nine patients. The absence of significant differences between the groups should be interpreted with caution. The use of a stable isotope gastric emptying breath test as a postoperative recovery marker is novel. Stable isotope gastric emptying breath testing requires no specialist technical skills and is well tolerated[40, 41]. This is an inexpensive method for determining the gastric emptying rate and may be an objective marker of recovery in the early postoperative period. The limitations of the test may preclude it from being suitable for comparing subjects with rapid gastric emptying. It may be better suited to highlighting patients who have delayed gastric emptying and are likely to have a slower return to full diet, longer hospital stay and slower postoperative recovery. In the present protocol, day 3 after surgery was chosen for this test because the patients had returned to the ward. It would be of interest to study this variable at other time points. Within the context of multimodal therapy it is difficult to separate the effect of one protocol component from another. A two-by-two factorial design is an efficient way of assessing two different factors and evaluating their potential interaction. The statistical design of the present study meant that there was a potential confounder in 50 per cent of each group under description; this reflects the exploratory nature of the evaluation of potential interactions.

\section{CONCLUSION}

The present study has shown that laxatives can hasten return of colonic function in patients undergoing hepatic resection. However, there was no evident interaction with ONS. More importantly, there seemed to be no benefit in terms of enhanced oral intake or overall recovery. Thus the benefit of routine laxatives or ONS is difficult to gauge. Clearly the present findings cannot be extrapolated directly to patients with an intestinal anastomosis. Future studies might also evaluate the interaction between the present variables and other measures designed to improve upper gastrointestinal function and oral intake. 


\section{REFERENCES}

1. Desborough, J.P., The stress response to trauma and surgery. Br J Anaesth, 2000. 85(1): p. 109-17.

2. Nygren, J., et al., A comparison in five European Centres of case mix, clinical management and outcomes following either conventional or fast-track perioperative care in colorectal surgery. Clin Nutr, 2005. 24(3): p. 45561.

3. Fearon, K.C., et al., Enhanced recovery after surgery: a consensus review of clinical care for patients undergoing colonic resection. Clin Nutr, 2005. 24(3): p. 466-77.

4. van Dam, R.M., et al., Initial experience with a multimodal enhanced recovery programme in patients undergoing liver resection. Br J Surg, 2008. 95(8): p. 969-75.

5. Podore, P.C. and E.B. Throop, Infrarenal aortic surgery with a 3-day hospital stay: A report on success with a clinical pathway. J Vasc Surg, 1999. 29(5): p. 787-92.

6. Cerfolio, R.J., et al., Fast tracking after Ivor Lewis esophagogastrectomy. Chest, 2004. 126(4): p. 1187-94.

7. Basse, L., et al., A clinical pathway to accelerate recovery after colonic resection. Ann Surg, 2000. 232(1): p. 51-7.

8. Wind, J., et al., Systematic review of enhanced recovery programmes in colonic surgery. Br J Surg, 2006. 93(7): p. 800-9.

9. Walter, C.J., et al., Enhanced recovery in colorectal resections: a systematic review and meta-analysis. Colorectal Dis, 2009. 11(4): p. 344-53.

10. Belghiti, J., et al., Seven hundred forty-seven hepatectomies in the 1990s: an update to evaluate the actual risk of liver resection. J Am Coll Surg, 2000. 191(1): p. 38-46.

11. Jarnagin, W.R., et al., Improvement in perioperative outcome after hepatic resection: analysis of 1,803 consecutive cases over the past decade. Ann Surg, 2002. 236(4): p. 397-406; discussion 406-7.

12. Dimick, J.B., et al., National trends in the use and outcomes of hepatic resection. J Am Coll Surg, 2004. 199(1): p. 31-8.

13. Lobo, D.N., et al., Effect of salt and water balance on recovery of gastrointestinal function after elective colonic resection: a randomised controlled trial. Lancet, 2002. 359(9320): p. 1812-8.

14. Nisanevich, V., et al., Effect of intraoperative fluid management on outcome after intraabdominal surgery. Anesthesiology, 2005. 103(1): p. 25-32.

15. Fotiadis, R.J., et al., Epidural analgesia in gastrointestinal surgery. Br J Surg, 2004. 91(7): p. 828-41.

16. Stewart, B.T., et al., Early feeding after elective open colorectal resections: a prospective randomized trial. Aust N Z J Surg, 1998. 68(2): p. 125-8.

17. Fanning, J. and S. Yu-Brekke, Prospective trial of aggressive postoperative bowel stimulation following radical hysterectomy. Gynecol Oncol, 1999. 73(3): p. 412-4.

18. Noblett, S.E., et al., Pre-operative oral carbohydrate loading in colorectal surgery: a randomized controlled trial. Colorectal Dis, 2006. 8(7): p. 563-9.

19. Soop, M., et al., Preoperative oral carbohydrate treatment attenuates immediate postoperative insulin resistance. Am J Physiol Endocrinol Metab, 2001. 280(4): p. E576-83.

20. Nygren, J., et al., Preoperative oral carbohydrate administration reduces postoperative insulin resistance. Clin Nutr, 1998. 17(2): p. 65-71.

21. Beattie, A.H., et al., A randomised controlled trial evaluating the use of enteral nutritional supplements postoperatively in malnourished surgical patients. Gut, 2000. 46(6): p. 813-8.

22. Smedley, F., et al., Randomized clinical trial of the effects of preoperative and postoperative oral nutritional supplements on clinical course and cost of care. Br J Surg, 2004. 91(8): p. 983-90.

23. Strasberg, S.M.e.a., The Brisbane 2000 terminology of liver anatomy and resections. HPB (Oxford), 2000. 2: p. 333-39.

24. Braden, B., et al., The [13C]acetate breath test accurately reflects gastric emptying of liquids in both liquid and semisolid test meals. Gastroenterology, 1995. 108(4): p. 1048-55.

25. Slater, C., T. Preston, and L.T. Weaver, Comparison of 
accuracy and precision of heart rate calibration methods to estimate total carbon dioxide production during 13C-breath tests. Eur J Clin Nutr, 2006. 60(1): p. 69-76.

26. Preston, T. and D.C. McMillan, Rapid sample throughput for biomedical stable isotope tracer studies. Biomed Environ Mass Spectrom, 1988. 16(1-12): p. 229-35.

27. Siegel, J.A., et al., Biphasic nature of gastric emptying. Gut, 1988. 29(1): p. 85-9.

28. Bluck, L.J. and W.A. Coward, Measurement of gastric emptying by the $13 \mathrm{C}$-octanoate breath test--rationalization with scintigraphy. Physiol Meas, 2006. 27(3): p. 279-89.

29. Lang, M., et al., Outcome and resource utilization in gastroenterological surgery. Br J Surg, 2001. 88(7): p. 1006-14.

30. Copeland, G.P., D. Jones, and M. Walters, POSSUM: a scoring system for surgical audit. Br J Surg, 1991. 78(3): p. $355-60$.

31. Zingg, U., et al., Effect of bisacodyl on postoperative bowel motility in elective colorectal surgery: a prospective, randomized trial. Int J Colorectal Dis, 2008. 23(12): p. $1175-83$.

32. Hansen, C.T., et al., Effect of laxatives on gastrointestinal functional recovery in fast-track hysterectomy: a double-blind, placebo-controlled randomized study. Am J Obstet Gynecol, 2007. 196(4): p. 311 e1-7.

33. Petrowsky, H., et al., A prospective, randomized, controlled trial comparing intermittent portal triad clamping versus ischemic preconditioning with continuous clamping for major liver resection. Ann Surg, 2006. 244(6): p. 921-8; discussion 928-30.

34. Capussotti, L., et al., Randomized clinical trial of liver resection with and without hepatic pedicle clamping. $\mathrm{Br}$ J Surg, 2006. 93(6): p. 685-9.

35. Maessen, J., et al., A protocol is not enough to implement an enhanced recovery programme for colorectal resection. Br J Surg, 2007. 94(2): p. 224-31.

36. Nikfarjam, M., et al., Survival outcomes of patients with colorectal liver metastases following hepatic resection or ablation in the era of effective chemotherapy. Ann
Surg Oncol, 2009. 16(7): p. 1860-7.

37. Fong, Y., et al., Drainage is unnecessary after elective liver resection. Am J Surg, 1996. 171(1): p. 158-62.

38. Burt, B.M., et al., An audit of results of a no-drainage practice policy after hepatectomy. Am J Surg, 2002. 184(5): p. 441-5.

39. Hendry, P.O., et al., Determinants of outcome after colorectal resection within an enhanced recovery programme. Br J Surg, 2009. 96(2): p. 197-205.

40. Ghoos, Y.F., et al., Measurement of gastric emptying rate of solids by means of a carbon-labeled octanoic acid breath test. Gastroenterology, 1993. 104(6): p. 1640-7.

41. Perri, F., et al., 13C-octanoic acid breath test: a reliable tool for measuring gastric emptying. Ital J Gastroenterol Hepatol, 1998. 30(2): p. 211-7. 

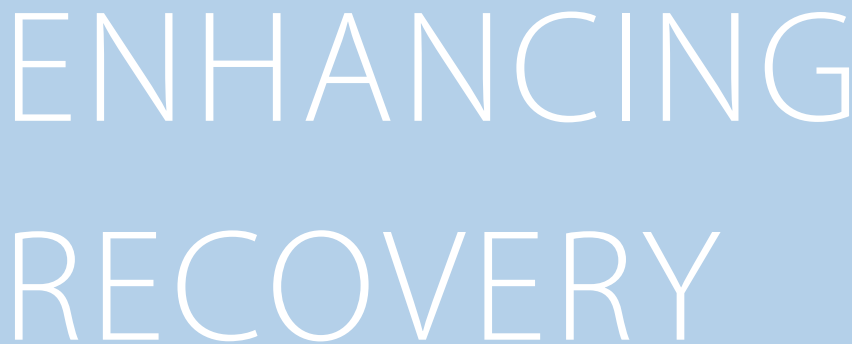

\section{PUBLISHED AS}

A survey in the hepatopancreatobiliary community on ways to enhance patient recovery

E.M. Wong-Lun-Hing, T.M. Lodewick, J.H.M.B. Stoot, M.H.A. Bemelmans, S.W.M. Olde Damink, C.H.C. Dejong, R.M. van Dam

HPB 2012; 14 (12): 818-827

DOI: dx.doi.org/10.1111/j.1477-2574.2012.00546.x 


\section{ABSTRACT}

\section{OBJECTIVE}

Both laparoscopic techniques and multimodal enhanced recovery programmes have been shown to improve recovery and reduce length of hospital stay. Interestingly, evidence-based care programmes are not widely implemented, whereas new, minimally invasive surgical procedures are often adopted with very little evidence to support their effectiveness. The present survey aimed to shed light on experiences of the adoption of both methods of optimizing recovery.

\section{METHODS}

An international, web-based, 18-question, electronic survey was composed in 2010. The survey was sent out to 673 hepatopancreatobiliary (HPB) centres worldwide in June 2010 to investigate international experiences with laparoscopic liver surgery, fast-track recovery programmes and surgery-related equipoise in open and laparoscopic techniques and to assess opinions on strategies for adopting laparoscopic liver surgery in HPB surgical practice.

A total of 507 centres responded (response rate: 75.3\%), 161 of which finished the survey completely. All units reported performing open liver resections, $24.2 \%$ performed open living donor resections, 39.1\% carried out orthotopic liver transplantations, $87.6 \%$ had experience with laparoscopic resections and $2.5 \%$ performed laparoscopic living donor resections. A median of 50 (range: 2-560) open and 9.5 (range: 1-80) laparoscopic liver resections per surgical unit were performed in 2009. Patients stayed in hospital for a median of 7 days (range: 2-15 days) after uncomplicated open liver resection and a median of 4 days (range: 1-10 days) after uncomplicated laparoscopic liver resection. Only $28.0 \%$ of centres reported having experience with fast-track programmes in liver surgery. The majority considered the instigation of a randomized controlled trial or a prospective register comparing the outcomes of open and laparoscopic techniques to be necessary.

\section{CONCLUSION}

Worldwide dissemination of laparoscopic liver resection is substantial, although laparoscopic volumes are low in the majority of HPB centres. The adoption of enhanced recovery programmes in liver surgery is limited and should be given greater attention. 


\section{INTRODUCTION}

In recent years, laparoscopic liver resection and enhanced recovery programmes have been introduced in liver surgery with the aim of accelerating postoperative recovery and shortening hospital length of stay (LoS). Like open liver resection, laparoscopic resection of liver lesions can be applied safely in both malignant and benign disease[1-7]. Large prospective case series suggest that laparoscopic liver surgery may be superior to open liver surgery in terms of perioperative blood loss, postoperative pain, time to recovery, LoS, cosmetic appearance and quality of life[5, 8-10]. Survival rates after laparoscopic and open resection of hepatocellular carcinoma and hepatic colorectal metastases seem to be comparable[1, 5]. Similarly, fast-track programmes have proven to be useful, feasible and safe in liver surgery[11-15]. Such programmes can also enhance recovery and reduce LoS. Enhanced recovery after surgery (ERAS) programmes rely mainly on optimizing perioperative care and reducing stress responses to surgery through the provision of adequate preoperative patient counselling, optimized anaesthesia and analgesia, quick resumption of oral intake and early mobilization[12, 16-18]. In liver resection, earlier resumption of oral intake, faster postoperative recovery and a significant reduction in median LoS (from 8 days to 6 days) was shown when patients were managed within a multimodal ERAS programme[12].

A small pilot study in liver surgery suggested that laparoscopic liver surgery within an ERAS setting led to a potentially accelerated recovery and further reduction in LoS from 7 days to 5 days[11]. Moreover, the combining of laparoscopy and an ERAS strategy is most likely to result in a synergetic effect, as recently proven in a randomized controlled trial (RCT) in the context of colonic surgery[19].

Despite the fairly robust evidence that many specific elements of fast-track programmes can enhance recovery and reduce LoS, little evidence on the use of these programmes in liver surgery has been published. This suggests that the adoption of ERAS programmes in liver surgery worldwide is low. Current surgical practice is based on evidence and any change in daily routines should be supported by sound data[20]. In this respect, the current fairly liberal adoption of laparoscopic liver surgery contrasts with the relative lack of adoption of enhanced recovery programmes.

An international web-based survey was composed to evaluate worldwide experiences with laparoscopic liver surgery and fast-track recovery programmes, and surgery-related equipoise in open and laparoscopic strategies, and to assess opinions on strategies for the adoption of laparoscopic liver surgery in daily hepatopancreatobiliary (HPB) surgical practice. 


\section{METHODS}

\section{STUDY DESIGN}

An online electronic survey, consisting of 18 questions subdivided according to five main domains, was developed (Appendix 1). Questions on the different topics were initially composed by two research fellows (EMWLH and TML) and two liver surgeons (RMvD and JHMBS). The definitive set of questions was then administered using SurveyMonkey ${ }^{\mathrm{TM}}$ (SurveyMonkey, Inc., Palo Alto, CA, USA). Items in the first domain included several ques-tions on experience in open and laparoscopic liver surgery and covered types and numbers of liver resections. Items in the second domain concerned recovery and LoS after uncomplicated open or laparoscopic liver resection. Items in the third domain surveyed experience with enhanced recovery or fast-track perioperative care programmes such as ERAS programmes. The fourth part of the survey was developed to evaluate opinions on the necessity of an RCT and on the value of a prospective registry comparing outcomes in open and laparoscopic liver surgery. Items in the final domain evaluated current opinions on and considerations for participating in such a trial. Incomplete responses were excluded from analysis.

\section{STUDY POPULATION}

An invitation to complete this survey was sent by e-mail to 673 HPB centres worldwide in June 2010. Subsequent e-mail reminders were sent out in August and September 2010. Only one surgeon per HPB unit was asked to participate. Figure 1 describes the respondent inclusion and exclusion process. The participation period closed and analyses were conducted in November 2010.

\section{STATISTICS}

Survey data were extracted into an Excel database. Statistical analysis was performed using SPSS Version 18.0 (SPSS, Inc., Chicago, IL, USA) and GraphPad Prism Version 5 (GraphPad Software, Inc., La Jolla, CA, USA). Basic analyses were performed using descriptive statistics including counts, percentages, means with standard deviations and medians with ranges and interquartile ranges (IQRs). Subgroup analysis was performed to investigate potentially relevant differences among regions and centre experiences using the Mann-Whitney U-test or chi-squared test. All countries were assigned to one of the following six regions: Europe; North America; Central and South America; Asia; Oceania, and Africa. An experienced laparoscopic HPB centre was defined as a surgical unit performing 20 or more laparoscopic liver resections annually[21]. 


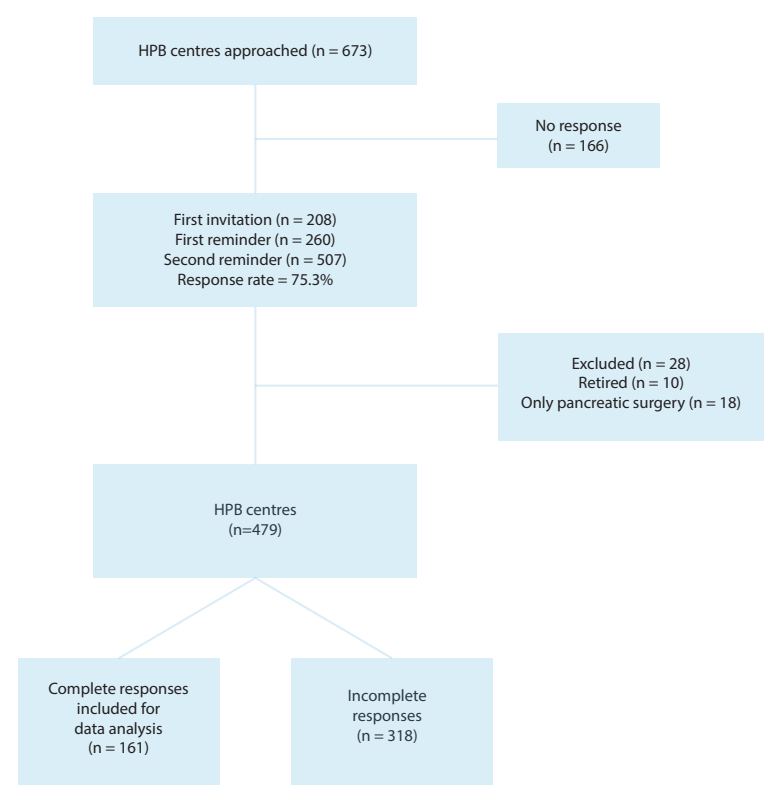

Figure 1 Flowchart showing the respondent inclusion and exclusion process. A total of 673 hepatopancreatobiliary (HPB) centres were approached. Cumulative response numbers after the first invitation and first and second reminders are displayed. A total of 166 surgeons did not respond to the study invitation or reminders, and data from an additional 28 centres were excluded. Only data from the centres that provided complete responses $(n=161)$ were analysed

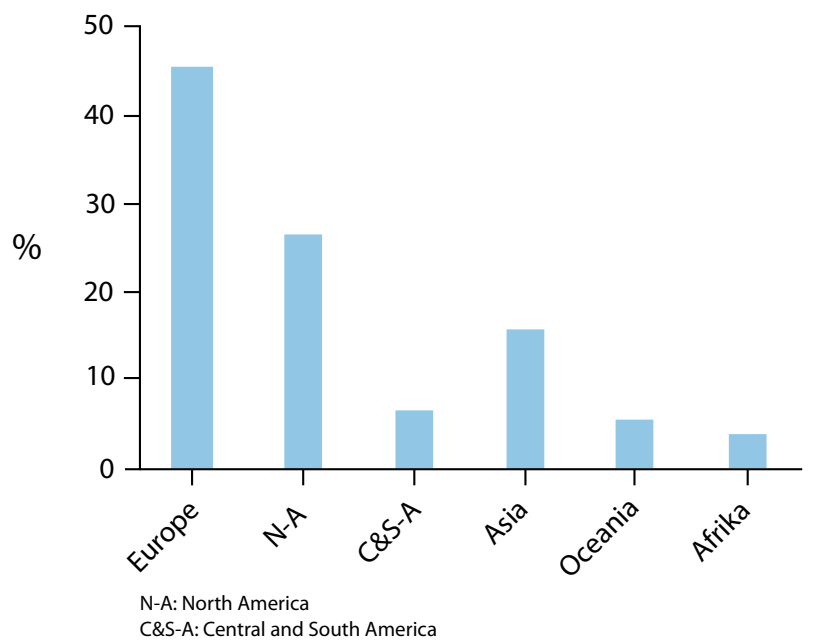

Figure 2 Centres $(n=161)$ included in the data analysis by region 


\section{RESULTS}

\section{PARTICIPATION}

A total of 507 centres (one surgeon per centre) responded (response rate $75.3 \%$ ). Incomplete responses were excluded from data analysis, leaving complete responses from 161 centres. Centres in 39 different countries participated; these were divided into groups according to the six global regions (Fig. 2). The regions that provided the highest response rates were Europe (45\%) and North America (26\%), with the USA $(n=34)$, Italy $(n=16)$, Canada $(n=8)$ and the Netherlands $(n=8)$ representing the top four countries providing complete responses.

\section{TYPES OF LIVER RESECTION}

Open resection of liver lesions was performed by $100 \%$ of the units. Overall, $87.6 \%$ of responding units reported experience with laparoscopic resection of liver lesions, $39.1 \%$ with orthotopic liver transplantation, $24.2 \%$ with open living donor resection and $2.5 \%$ with laparoscopic living donor resection. Table 1 shows the percentages of HPB centres performing different types of liver surgery and the differences among regions.

\begin{tabular}{|c|c|c|c|c|c|c|c|}
\hline \multicolumn{8}{|c|}{ Centres performing surgical procedures, by region, $n(\%)$} \\
\hline & Europe & $\begin{array}{l}\text { North } \\
\text { America }\end{array}$ & $\begin{array}{c}\text { Central } \\
\text { and South } \\
\text { America }\end{array}$ & Asia & Oceania & Africa & Worldwide \\
\hline Open resection of liver lesions & 72 & 42 & 10 & 24 & 8 & 5 & $161(100.0)$ \\
\hline $\begin{array}{l}\text { Laparoscopic resection of liver } \\
\text { lesions }\end{array}$ & 61 & 41 & 10 & 19 & 7 & 3 & $141(87.6)$ \\
\hline Orthotopic liver transplantation & 29 & 17 & 5 & 7 & 4 & 1 & $63(39.1)$ \\
\hline Open living donor resection & 15 & 10 & 4 & 7 & 2 & 1 & $39(24.2)$ \\
\hline $\begin{array}{l}\text { Laparoscopic living donor } \\
\text { resection }\end{array}$ & 1 & 1 & 1 & 1 & 0 & 0 & $4(2.5)$ \\
\hline
\end{tabular}

\section{EXPERIENCE}

A total of $42.0 \%$ of responding centres indicated that their data represented precise numbers. The remaining centres provided estimations that were as accurate as possible. A wide range in the number of resections performed was observed among HPB centres. In 2009, the median number of open resections for liver lesions performed was 50 (range: 2-560; $I Q R=50$ ) per surgical unit. In the same year, the median number of laparoscopic resections of liver lesions performed was 9.5 (range: 1-80; IQR = 15) per centre. Worldwide figures for open and laparoscopic liver resections and differences among regions are shown in Table 2. Of the participating centres, $26.6 \%$ could be classified as experienced laparoscopic liver centres based on their completion of at least 20 laparoscopic liver resections per year (Table 3). Experienced laparoscopic centres seemed to be more frequently located in the Americas than in other continents ( $44.2 \%$ vs. $13.5 \% ; \mathrm{P}<0.001)$. 


\begin{tabular}{|c|c|c|}
\hline \multirow[t]{2}{*}{ Region } & \multicolumn{2}{|c|}{ Liver resections performed in 2009, median (range)/IQR } \\
\hline & Open liver resections & Laparoscopic liver resections \\
\hline Europe & $55.0(2-250) / 60.0$ & $5.0(1-61) / 9.0$ \\
\hline North America & $45.0(6-200) / 42.5$ & $19.0(2-80) / 21.0$ \\
\hline Central and South America & $32.5(12-80) / 23.5$ & $10.0(3-30) / 18.0$ \\
\hline Asia & $50.0(5-560) / 62.3$ & $6.0(1-80) / 5.0$ \\
\hline Oceania & $57.5(15-150) / 110.0$ & $9.0(1-20) / 7.0$ \\
\hline Africa & $50.0(5-120) / 115.0$ & $4.5(3-13) / 7.8$ \\
\hline Worldwide & $50.0(2-560) / 50.0$ & $9.5(1-80) / 15.0$ \\
\hline
\end{tabular}

Table 2 Number of resections per surgical unit per year (2009)

\section{HOSPITAL LENGTH OF STAY AND FAST-TRACK PERIOPERATIVE CARE PROGRAMMES}

The reported median hospital LoS after uncomplicated liver resection was 7 days (range: 2-15 days) after open surgery and 4 days (range: 1-10 days) after laparoscopic surgery. Differences among regions are shown in Table 4. In addition, a subgroup analysis showed that experienced centres achieved a significantly shorter median LoS after laparoscopic liver resection compared with inexperienced centres [3.4 days (range: 1-7 days) vs. 4.2 days (range: $1-10$ days); $P=0.013]$. Half of the HPB centres $(50.1 \%)$ had experience with fast-track perioperative care programmes in colonic, hepatic or pancreatic surgery or a combination of these fields. Only $28.0 \%$ had experience with these programmes in liver surgery. Results per region are shown in Table 5.

\begin{tabular}{|l|r|}
\hline Region & $\begin{array}{l}\text { Experienced laparoscopic liver centres, } n \text { /laparoscopic liver } \\
\text { centres, } n(\%)\end{array}$ \\
\hline Europe & $10 / 59(16.9 \%)$ \\
\hline North America & $20 / 41(48.8 \%)$ \\
\hline Central and South America & $3 / 9(33.3 \%)$ \\
\hline Asia & $3 / 19(15.8 \%)$ \\
\hline Oceania & $1 / 7(14.3 \%)$ \\
\hline Africa & $0 / 4$ \\
\hline Worldwide & $37 / 139(26.6 \%)$ \\
\hline
\end{tabular}

Table 3 Numbers of laparoscopic liver surgery centres demonstrating experience defined by a volume of $>20$ laparoscopic resections per year

\section{NECESSITY FOR AN RCT COMPARING OPEN AND LAPAROSCOPIC LIVER SURGERY}

The majority (59.4\%) of HPB centres considered that an RCT comparing outcomes in open and laparoscopic liver surgery prior to the further adoption of laparoscopic liver surgery was necessary. A total of $49.1 \%$ considered that a combination of such an RCT and a pro- 
spective multicentre registry should be mandatory; $36.4 \%$ considered that a prospective multicentre registry alone would be sufficient and $4.2 \%$ deemed a prospective hospital reg-istry adequate. Of the surgical units that performed both open and laparoscopic left lateral sectionectomy (LLS) within a fasttrack/ERAS programme, $82.9 \%$ indicated that they would consider participating in an RCT. Level A evidence to support the superiority of the laparoscopic technique was still considered necessary by the majority of respondents. A total of $94.3 \%$ of participants with experience in both open and laparoscopic LLS, both within and outwith fast-track or ERAS programmes, would also consider participating in a prospective registry. Overall, $83.3 \%$ of liver units without experience in laparoscopic liver surgery indicated a desire to participate in hands-on training in laparoscopic liver surgery and/or a proctor programme.

\begin{tabular}{|c|c|c|}
\hline \multirow[t]{2}{*}{ Region } & \multicolumn{2}{|l|}{ Lenght of stay, days, median (range)/IQR } \\
\hline & After open resection & After laparoscopic resection \\
\hline Europe & $7.0(4-12) / 3.0$ & $5.0(2-10) / 1.0$ \\
\hline North America & $5.0(4-8) / 2.0$ & $3.0(1-5) / 1.5$ \\
\hline Central and South America & $5.0(2-7) / 1.8$ & $2.5(1-5) / 2.0$ \\
\hline Asia & $7.0(5-15) / 2.0$ & $4.0(3-10) / 2.0$ \\
\hline Oceania & $5.0(3-7) / 1.8$ & $4.0(2-5) / 1.5$ \\
\hline Africa & $8.0(4-10) / 5.5$ & $4.5(2-7) / 4.5$ \\
\hline Worldwide & $7.0(2-15) / 3.0$ & $4.0(1-10) / 2.0$ \\
\hline
\end{tabular}

Table 4 Length of stay after uncomplicated liver resection

\begin{tabular}{|c|c|c|c|c|}
\hline \multirow[t]{2}{*}{ Region } & \multicolumn{4}{|c|}{ Experience with ERAS programmes in a specific type of surgery, $n /$ total $n(\%)$} \\
\hline & No experience & In colon surgery & In liver surgery & In pancreatic surgery \\
\hline Europe & $31 / 72(43.1 \%)$ & $36 / 72(50.0 \%)$ & $22 / 72(30.6 \%)$ & $14 / 72(19.4 \%)$ \\
\hline North America & $25 / 42(59.5 \%)$ & $6 / 42(14.3 \%)$ & $9 / 42(21.4 \%)$ & $8 / 42(19.0 \%)$ \\
\hline Central and South America & $5 / 10(50.0 \%)$ & $4 / 10(40.0 \%)$ & $3 / 10(30.0 \%)$ & $1 / 10(10.0 \%)$ \\
\hline Asia & $15 / 24(62.5 \%)$ & $8 / 24(33.3 \%)$ & $4 / 24(16.7 \%)$ & $3 / 24(12.5 \%)$ \\
\hline Oceania & $2 / 8(25.0 \%)$ & $5 / 8(62.5 \%)$ & $6 / 8(75.0 \%)$ & $2 / 8(25.0 \%)$ \\
\hline Africa & $4 / 5(80.0 \%)$ & $1 / 5(20.0 \%)$ & $1 / 5(20.0 \%)$ & $0 / 5$ \\
\hline Worldwide & $82 / 161$ (50.9\%) & $60 / 161(37.3 \%)$ & $45 / 161(28.0 \%)$ & $28 / 161(17.4 \%)$ \\
\hline
\end{tabular}




\section{DISCUSSION}

This study aimed to assess the worldwide experience and dissemination of two recently introduced strategies to accelerate recovery after liver surgery. It demonstrates that the majority of HPB centres perform liver surgery in the absence of an enhanced recovery perioperative care programme, and that the majority of HPB centres perform laparoscopic liver surgery. This study also shows substantial variance in hospital LoS among centres and regions. Lastly, this study demonstrates the presence of clinical equipoise in laparoscopic and open liver resection in the HPB community[22,23]. Clinical equipoise refers to a context in which there is no preference or certainty of therapeutic superiority for either laparoscopic or open liver surgery. The majority of liver centres considered that an RCT was necessary to prove the laparoscopic technique to be equal or superior to open surgery.

The results show a high level of dissemination of laparoscopic liver surgery. Both low-and high-volume centres, amounting to $87.6 \%$ of HPB units, perform liver resections laparoscopically. Although the laparoscopic procedure is frequently used, many centres in this study have limited experience in laparoscopic liver resection. By contrast, responding centres displayed limited adoption of enhanced recovery programmes in liver surgery (one in four). The liberal adoption of laparoscopic liver surgery, even in low-volume HPB centres, is seemingly in conflict with current standards of evidence-based practice in the medical community. Neither is it in keeping with recommendations expressed in an expert consensus (the Louisville Consensus), which concluded that laparoscopic liver surgery was safe and effective in the hands of experienced and trained surgeons[24]. In line with the available evidence for fast-track principles in liver surgery proof of the merits of laparoscopic vs. open liver surgery is also limited and no RCTs have been undertaken[11-15]. However, a recent literature review and meta-analysis of available prospective and retrospective studies comparing open with laparoscopic liver resections both found short-and longterm outcomes favourable for the laparoscopic procedure. Not only was LoS markedly shorter, but blood loss and complications were found to be reduced and oncologic outcomes did not differ between the two techniques[25, 26].

Low-volume centres in this study reported a significantly longer hospital LoS compared with high-volume centres. In addition, LoS after open and laparoscopic liver surgery varied substantially among regions. The surgeon's progress along the laparoscopic learning curve influences LoS because laparoscopic liver resection is technically demanding and requires expertise in both advanced laparoscopic skills and open liver surgery[27]. This is in line with the findings of a meta-analysis of studies reporting on 20 or more laparoscopic procedures, which indicated that a laparoscopic approach led to a significant reduction in morbidity and LoS[21]. In addition, differences in standard of care and discharge criteria may also contribute to the variance in LoS reported in the literature (3-20 days after open and 6-32 days after 
laparoscopic liver resection)[25]. Substantial distinctions at a cultural level may lie at the root of the observed variance in LoS. In some regions patients are discharged to a home care institution early in their recovery process (e.g. in North America), whereas in other world regions the provision of protocol-based care and the associated logistics may be lacking. This may lead to a difference in expectations on both the patient's and surgeon's part as to when a patient might be ready for discharge. Thus, LoS is a poor outcome parameter that hampers comparison and is hard to interpret. The implementation of a structured care programme with well-defined recovery and discharge criteria, as used within ERAS protocols, might improve the comparability of clinical outcomes in future (multicentre) trials.

Laparoscopy and enhanced recovery programmes should not be seen as separate methods of improving postoperative recovery and outcomes such as morbidity rates and LoS. On the contrary, it is likely that the implementation of both will result in a synergetic improvement. Enhanced recovery programmes in liver surgery have already been shown to reduce hospital LoS[12-15]. The additional benefit of an ERAS strategy in a laparoscopic setting was recently demonstrated in a trial in colonic surgery and a small pilot study in liver surgery[11, 19]. This worldwide survey unveils experiences in enhanced recovery programmes and laparoscopic liver surgery, and deliberations on the need for RCTs in liver surgery. Although the present study achieved a response rate of $>75 \%$, only $23.9 \%$ of respondents completed the survey in full, which limited the study findings. However, representatives of 161 liver surgery centres worldwide shared their results and opinions.

The use of a survey may be seen as limiting the study because results are strongly dependent on the type of respondents, questions and response rate. In addition, the group profile of responding centres may have been subject to response bias. Centres of considerable volume and those performing laparoscopic liver surgery may have been more likely to respond.

The issue of importance does not concern a choice between laparoscopic liver resection majority of responders still consider an RCT of laparoscopic surgery to be necessary. It could be argued that as more liver centres adopt laparoscopic techniques, opportunities to conduct an RCT may be diminished by patient and surgeon preferences. According to some surgeons, laparoscopic liver resection is without doubt therapeutically superior, whereas for others this remains to be proven. In addition, in the Louisville Consensus of 2008, experts concluded that the accrual of patients for an RCT would be slow as a result of low overall numbers.

The ORANGE II Trial, currently enrolling patients, will be the first RCT (combined with a prospective registry) to provide evidence on laparoscopic vs. open liver resection[28]. As for enhanced recovery programmes, many centres are likely to have already implemented multiple ERAS elements as part of modern care. Further trials are needed to assess compliance 
with recovery protocols[29, 30], specific elements of enhanced recovery programmes and the possible reduction of medical expenses.

\section{CONCLUSION}

The worldwide dissemination of laparoscopic liver resection is substantial, although the average volume of laparoscopic resections carried out in the majority of HPB centres is low. The adoption of enhanced recovery programmes in liver surgery is limited and the issue warrants greater attention. Both strategies are associated with faster recovery and may work synergistically. Given the increasing strength of the role of evidence-based medicine in current surgical practice, more evidence is required. 


\section{REFERENCES}

1. Nguyen, K.T., T.C. Gamblin, and D.A. Geller, World review of laparoscopic liver resection-2,804 patients. Ann Surg, 2009. 250(5): p. 831-41.

2. Abu Hilal, M. and N.W. Pearce, Laparoscopic left lateral liver sectionectomy: a safe, efficient, reproducible technique. Dig Surg, 2008. 25(4): p. 305-8.

3. Dagher, I., et al., Laparoscopic liver resection for hepatocellular carcinoma. Surg Endosc, 2008. 22(2): p. 372-8.

4. Dagher, l., et al., Laparoscopic liver resection: results for 70 patients. Surg Endosc, 2007. 21(4): p. 619-24.

5. Lee, K.F., et al., Laparoscopic versus open hepatectomy for liver tumours: a case control study. Hong Kong Med J, 2007. 13(6): p. 442-8.

6. O'Rourke, N. and G. Fielding, Laparoscopic right hepatectomy: surgical technique. J Gastrointest Surg, 2004. 8(2): p. 213-6.

7. Troisi, R., et al., The value of laparoscopic liver surgery for solid benign hepatic tumors. Surg Endosc, 2008. 22(1): p. 38-44.

8. Laurence, J.M., et al., Laparoscopic hepatectomy, a systematic review. ANZ J Surg, 2007. 77(11): p. 948-53.

9. Abu Hilal, M., et al., Laparoscopic versus open left lateral hepatic sectionectomy: A comparative study. Eur J Surg Oncol, 2008. 34(12): p. 1285-8.

10. Alkari, B., A. Owera, and B.J. Ammori, Laparoscopic liver resection: preliminary results from a UK centre. Surg Endosc, 2008. 22(10): p. 2201-7.

11. Stoot, J.H., et al., The effect of a multimodal fast-track programme on outcomes in laparoscopic liver surgery: a multicentre pilot study. HPB (Oxford), 2009. 11(2): p. 140-4.

12. van Dam, R.M., et al., Initial experience with a multimodal enhanced recovery programme in patients undergoing liver resection. Br J Surg, 2008. 95(8): p. 969-75.

13. Lin, D.X., et al., Implementation of a fast-track clinical pathway decreases postoperative length of stay and hospital charges for liver resection. Cell Biochem Biophys, 2011. 61(2): p. 413-9.
14. MacKay, G. and P.J. O'Dwyer, Early discharge following liver resection for colorectal metastases. Scott Med J, 2008. 53(2): p. 22-4.

15. Spelt, L., et al., Fast-track programmes for hepatopancreatic resections: where do we stand? HPB (Oxford), 2011. 13(12): p. 833-8.

16. Kehlet, H. and D.W. Wilmore, Multimodal strategies to improve surgical outcome. Am J Surg, 2002. 183(6): p. 630-41.

17. Lassen, K., et al., Consensus review of optimal perioperative care in colorectal surgery: Enhanced Recovery After Surgery (ERAS) Group recommendations. Arch Surg, 2009. 144(10): p. 961-9.

18. Wind, J., et al., Systematic review of enhanced recovery programmes in colonic surgery. Br J Surg, 2006. 93(7): p. 800-9.

19. Vlug, M.S., et al., Laparoscopy in Combination with Fast Track Multimodal Management is the Best Perioperative Strategy in Patients Undergoing Colonic Surgery: A Randomized Clinical Trial (LAFA-study). Ann Surg, 2011.

20. Ubbink, D.T. and D.A. Legemate, Evidence-based surgery. Br J Surg, 2004. 91(9): p. 1091-2.

21. Simillis, C., et al., Laparoscopic versus open hepatic resections for benign and malignant neoplasms--a meta-analysis. Surgery, 2007. 141(2): p. 203-211.

22. Freedman, B., Equipoise and the ethics of clinical research. N Engl J Med, 1987. 317(3): p. 141-5.

23. Rodrigues, H.C., A.J. Oerlemans, and P.P. van den Berg, [The need for uncertainty in clinical researchEquipoise]. Ned Tijdschr Geneeskd, 2011. 155(49): p. A3846.

24. Buell, J.F., et al., The international position on laparoscopic liver surgery: The Louisville Statement, 2008. Ann Surg, 2009. 250(5): p. 825-30.

25. Mirnezami, R., et al., Short- and long-term outcomes after laparoscopic and open hepatic resection: systematic review and meta-analysis. HPB (Oxford), 2011. 13(5): p. 295-308.

26. Nguyen, K.T., et al., Comparative benefits of laparoscopic vs open hepatic resection: a critical appraisal. Arch Surg, 2011. 146(3): p. 348-56. 
27. van Gulik, T., Open versus laparoscopic resection for liver tumours. HPB (Oxford), 2009. 11(6): p. 465-8.

28. van Dam, R.M., et al., Open versus laparoscopic left lateral hepatic sectionectomy within an enhanced recovery ERAS(R) programme (ORANGE II-trial): study protocol for a randomised controlled trial. Trials, 2012. 13: p. 54.

29. Maessen, J., et al., A protocol is not enough to implement an enhanced recovery programme for colorectal resection. Br J Surg, 2007. 94(2): p. 224-31.

30. Spanjersberg, W.R., et al., Fast track surgery versus conventional recovery strategies for colorectal surgery. Cochrane Database Syst Rev, 2011. 2: p. CD007635. 


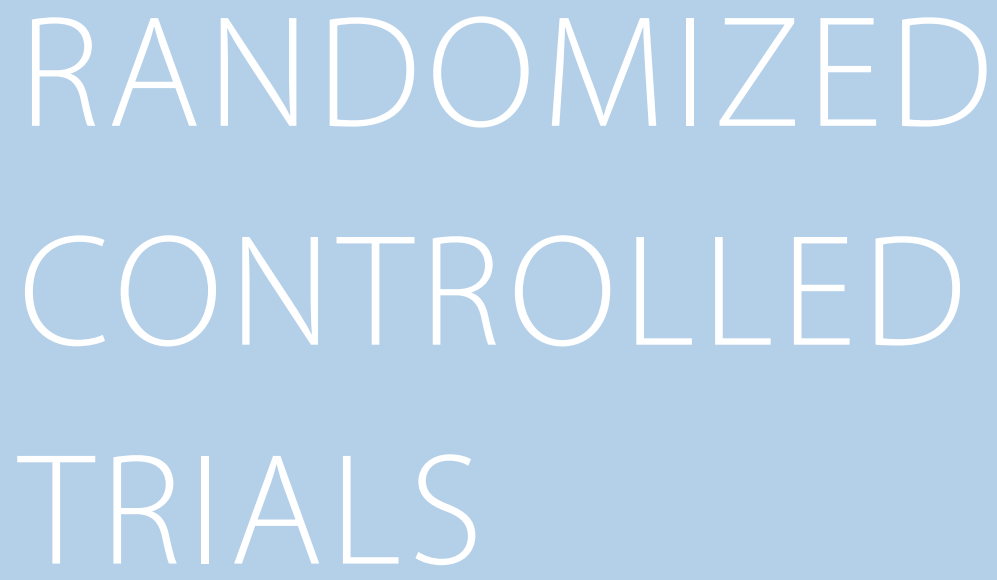

Laparoscopic vs open liver resection within an ERAS programme

\section{PUBLISHED AS}

Open versus laparoscopic left lateral hepatic sectionectomy within an enhanced recovery ERAS programme (ORANGE II-Trial): study protocol for a randomized controlled trial.

R.M. van Dam E.M. Wong-Lun-Hing, G.J.P. van Breukelen, J.H.M.B. Stoot, J.R. van der Vorst, M.H.A. Bemelmans, S.W.M. Olde Damink, K. Lassen, C.H.C. Dejong and ORANGE II Study Group

Trials 2012, http://www.trialsjournal.com/13/1/54

DOI: dx.doi.org/10.1186/1745-6215-13-54

Open versus laparoscopic hemi-hepatectomy within an enhanced recovery ERAS programme (ORANGE II PLUS-Trial): study protocol for a randomized controlled trial.

E.M. Wong-Lun-Hing, A. Laurent, I. Dagher, O. Scatton, O. Soubrane, U.P. Neumann, B. Topal, R. Troisi, L. Aldrighetti, J.N. Primrose, N.W. Pearce, M. Abu Hilal, D.P. Berry, G.J.P. van Breukelen, C.H.C. Dejong, R.M. van Dam and ORANGE II PLUS Study Group

Trials 2014, http://www.trialsjournal.com, submitted 


\section{ABSTRACT - ORANGE II TRIAL}

\section{BACKGROUND}

The use of laparoscopic liver resection in terms of time to functional recovery, length of hospital stay (LOS), long-term abdominal wall hernias, costs and quality of life (QOL) has never been studied in a randomised controlled trial. Therefore, this is the subject of the international multicentre randomised controlled ORANGE II trial.

\section{METHODS}

Patients eligible for left lateral sectionectomy (LLS) of the liver will be recruited and randomised at the outpatient clinic. All randomised patients will undergo surgery in the setting of an ERAS programme. The experimental design produces two randomised arms (open and laparoscopic LLS) and a prospective registry. The prospective registry will be based on patients that cannot be randomised because of the explicit treatment preference of the patient or surgeon, or because of ineligibility (not meeting the in-and exclusion criteria) for randomisation in this trial. Therefore, all non-randomised patients undergoing LLS will be approached to participate in the prospective registry, thereby allowing acquisition of an uninterrupted prospective series of patients. The primary endpoint of the ORANGE II trial is time to functional recovery. Secondary endpoints are postoperative LOS, percentage readmission, (liver-specific) morbidity, QOL, body image and cosmetic result, hospital and societal costs over 1 year, and long-term incidence of incisional hernias. It will be assumed that in patients undergoing laparoscopic LLS, length of hospital stay can be reduced by two days. A sample size of 55 patients in each randomisation arm has been calculated to detect a 2-day reduction in LOS (90\% power and $a=0.05$ (two-tailed)).

\section{CONCLUSION}

The ORANGE II trial is a multicenter randomised controlled trial that will provide evidence on the merits of laparoscopic surgery in patients undergoing LLS within an enhanced recovery ERAS programme.

ORANGE II TRIAL REGISTRATION: ClinicalTrials.gov NCT00874224. 


\section{BACKGROUND}

Liver resection for colorectal metastasis is the only potentially curative therapy, and has become the standard of care in appropriately staged patients, offering 5-year survival rates of approximately $35-40 \%$ [1]. For symptomatic benign lesions and those of uncertain nature or large size, liver resection is also a widely accepted treatment. Within the framework of optimising postoperative recovery and/ or producing a shorter length of stay (LOS) in hospital, laparoscopic surgery and enhanced recovery programmes have recently been introduced for liver surgery.

Laparoscopic liver resection was first described in 1995 [2]. Over the past decade the method has gained wide acceptance for various liver resection procedures [3-9]. Multiple retrospective case series and reviews comparing open with laparoscopic liver resection indicate that laparoscopic liver resection can be used safely for both malignant and benign liver lesions [10-15]. Recent publications from expert centers show that a substantial part of the total volume of major and minor liver resections is performed laparoscopically, and results are good $[16,17]$. Laparoscopic liver resection is associated with shorter LOS, less postoperative pain, earlier recovery, and better quality of life $(\mathrm{QOL})[9,13,18,19]$. Comparing patients undergoing an open left lateral sectionectomy (LLS) of the liver with those undergoing laparoscopic LLS, both Vigano et al. and Carswell et al. found no significant difference in operating time between the two groups[3, 4]. In addition, the median length of postoperative LOS was significantly less ( 6 vs. 9 days, $\mathrm{P}<0.01$ ) after laparoscopic resection [3].Furthermore, no evidence of a compromised oncologic clearance in laparoscopic liver resection has been found $[3,13]$ However, recovery and LOS are not only dependent on the type of surgery or procedure, and other variables should also be taken into account.

The Enhanced Recovery After Surgery (ERAS) programme has been introduced to improve postoperative care. This multimodal programme, derived from Kehlet's pioneer work in the 1990s for multimodal surgical care, involves optimisation of several aspects of the perioperative management of patients undergoing major abdominal surgery. In patients undergoing segmental colectomy, the ERAS programme enabled earlier recovery and consequently shorter LOS [20-23]. Furthermore, a reduction of post-operative morbidity in patients undergoing intestinal resection was reported [24-27]. These results stimulated liver surgeons of the ERAS group (Maastricht, Edinburgh and Tromsö) to adapt the ERAS programme to patients undergoing open liver resection. Van Dam et al. found a significantly reduced LOS after open liver resection when patients were managed within a multimodal ERAS programme[28]. Besides a reduction in median total LOS from 8 to 6 days (25\%), the data also suggested that a further reduction in stay could be possible as there was a delay between the recovery and actual discharge of the patients [28]. Moreover, Stoot et al. found retrospectively that there was a further reduction in LOS from 7 days to 5 days when patients were operated laparo- 
scopically and managed within an ERAS programme [29]. In that study there was also a delay between recovery and actual discharge of the patients. Previously, Maessen et al. reported a median delay to discharge of 2 days after patients had functionally recovered after colonic surgery managed within an ERAS programme [30]. This delay is often linked to patient age, hospital logistics, and absence of social and/or homecare support.

In most reported trials aiming at earlier recovery or a reduction in LOS, type of surgery and/ or perioperative management were not standardized. In addition, the added value of laparoscopic LLS compared with open left lateral sectionectomy within an ERAS programme in terms of time to functional recovery, LOS in hospital, costs, and QOL has never been studied in a randomized controlled trial (RCT). However, randomization of patients undergoing open or laparoscopic liver resection is hazardous. It is to be expected that experienced centres will be reluctant to randomize patients because of the absence of clinical and patient equipoise for laparoscopic resection. To capitalize on both centers with and without preference for laparoscopic liver surgery, and to thereby acquire an uninterrupted prospective series of patients, an alternative trial design with two randomization arms (open versus laparoscopic surgery) and a prospective registry has been constructed for the ORANGE II trial. The combination of an RCT and a prospective registry will improve overall power and strengthen the external validity and generalisability of study results [31-33].

\section{METHODS}

\section{ETHICS APPROVAL}

The study has been approved by the Medical Ethical Review Board of the Maastricht University Medical Center, Maastricht, The Netherlands trial number NL 25591.068.08 / MEC 08-2110. Ethics consent will also be obtained from the national or regional ethics boards in each participating country. Patients willing to participate in this trial will receive both verbal and written information at the time of recruitment in the outpatient clinic. In accordance with the local medical ethics committee all participating sites will provide an independent surgeon or physician if needed. An independent surgeon (M. Poeze) has been appointed for the Maastricht University Medical Center to answer questions. Confidentiality is guaranteed by assigning the participators an encoded trial number. This indicates that only the physician with the decoding 'key' will know which code number has been assigned to any patient. All trial data will be saved during the trial and stored on a server, and patients will be asked to consent to future analysis of these data. Withdrawal from the trial at any time or for any reason will not hold any form of consequences for the patient, and data from these patients will be deleted. 


\section{STUDY DESIGN}

The ORANGE II trial is a prospective superiority study with an experimental design, using two double-blinded randomized controlled arms and a prospective registry to determine whether laparoscopic surgery is to be preferred over open surgery in patients undergoing a LLS and participating in an enhanced recovery programme. In the participating randomizing centers, patients, nurses and the ward physician (but not the operating surgeon) will be blinded for the type of intervention up to and including postoperative day (POD) 3. They will record the functional recovery criteria twice daily. Only the investigator and operating surgeons will know the actual procedure. The blinded ward physician(s) will decide on whether a patient will be discharged or not. However, randomization of patients undergoing open or laparoscopic liver resection is hazardous as previously explained. Moreover, another potential source of bias exists when randomizing patients with a strong treatment preference. When patients cannot be blinded to their treatment allocation (POD 3) they may be resentful and demoralized if they do not receive their preferred treatment, and consequently they may have poor compliance. By contrast, patients receiving their preferred treatment may have above-average compliance. Thus to capitalize on centres both with and without preference for laparoscopic liver surgery, and thereby to acquire an uninterrupted prospective series of patients, all non-randomized patients undergoing a LLS will be approached to participate in the prospective registry. Registration of these patients is imperative to guarantee a consecutive series of patients and also because the absence of such a series may restrict generalization of the results, as randomized participants may not in fact be representative [16]. The combination of an RCT and a prospective registry will improve overall power and strengthen the external validity and generalizability of study results [31-33].This non-randomized registry group will be analyzed for centre and centre by treatment interaction as an observational study. Medical centres that wish to participate in this trial, but with liver surgeons early in the laparoscopic learning curve, will be accompanied during the procedure by an experienced proctoring laparoscopic HPB-surgeon.

\section{PRIMARY \& SECONDARY ENDPOINTS}

The primary endpoint of the ORANGE II trial is time to functional recovery. A patient is fully functionally recovered when all of the following five criteria are satisfied: 1 ) adequate pain control with oral analgesia; 2) restoration of mobility to an independent level; 3) absence of intravenous fluid administration; 4) ability to eat solid foods; and normal or decreasing serum bilirubin level and international normalized ratio. It is medically justified to discharge patients when the criteria for full functional recovery are met and if the patient is willing to go home. Secondary endpoints include postoperative LOS in hospital, percentage of read-missions, total morbidity (both general and procedure related), composite endpoint of liver-surgery-specific morbidity, QOL, body image and cosmesis, reasons for delay of discharge after functional recovery, hospital and societal costs over 1 year, and long-term incidence of incisional hernias. 


\section{MORBIDITY}

The preoperative morbidity status of patients will be measured using the American Society of Anesthesiologists (ASA) scale. The Portsmouth modification of the Physiological and Operative Severity Score for the Enumeration of Mortality and Morbidity (P-POSSUM) will be used to evaluate the risk of perioperative morbidity and mortality. Post-operative morbidity is rationally predictable, with hemorrhagic complications occurring predominantly during surgery or in the early postoperative phase, and biliary complications, intra-abdominal abscess, or liver failure in the later postoperative phase. Wound infection and sepsis will be additional complications that require monitoring. Morbidity will be classified and analyzed according to the validated classification for postoperative morbidity as described by Dindo et al [34].

\section{LIVER RESECTION-SPECIFIC COMPOSITE ENDPOINT}

In this trial, we will also use a well-defined liver surgery-specific composite endpoint, as suggested by van den Broek et al. [35]. This endpoint is a parameter composed of a combination of procedure-specific complications, which is considered as a single, dichotomous outcome: operative mortality, intra-abdominal haemorrhage, ascites, bile leakage, intra-abdominal abscess, and post-resectional liver failure. These components, which are all specific to liver surgery and have substantial clinical relevance, reflect complications rated as Dindo grade 3-5. A composite score of 1 (failure) will reflect the occurrence of at least one of the above liver-specific complications, and a score of 0 (success) will be assigned if none of these occur.

\section{QUALITY OF LIFE}

To assess QOL in patients undergoing laparoscopic versus open LLS, the Dutch version of the EuroQol five-dimension (EQ-5D) status test in Dutch centers and the translated EQ-5D for international centers will be used. The EQ-5D is a standardized instrument for use as a measure of health outcome, which consists of the five dimensions of mobility, self-care, usual activities, pain/ discomfort, and anxiety/depression, with three levels each and a rating on the EQ visual analogue scale (VAS; 0-100)[36-38]. Furthermore, the European Organization for Research and Treatment (EORTC) 30 item post-cancer QOL questionnaire (QLQ-C30; with the liver metastases (LM21) module will be used for liver-specific treatment measurements [39]. Assessment of the patients' QOL will be performed at the time of consent, discharge and 10 days, 3, 6 and 12 months after discharge.

\section{SBODY IMAGE AND COSMESIS}

To evaluate differences in postoperative body image and cosmesis, the Body Image Questionnaire (BIQ) will be used, which consists of eight questions about body image and cosmesis[40, 41]. The body image assessment will be performed preoperatively at time of consent. Both the body image and cosmesis assessment will take place at discharge, and at 10 days, 3 months, 6 months and 12 months after discharge. 


\section{HOSPITAL AND SOCIETAL COSTS}

The economic evaluation will include a cost-utility analysis from a Dutch societal perspective. The incremental costs per quality adjusted life year (QALY) gained will be based on utility scores from the EQ-5D [36-38]. All hospital expenses (direct and indirect) related to both interventions will be monitored. In addition, a cost questionnaire offered at the regular follow-up consultation (3, 6 and 12 months) will help assess the societal and individual costs outside health care relating to patients' absence, impaired mobility, work, or normal daily activities. Unit prices will be based either on prices from the participating hospital financial departments or will be extrapolated using Dutch guidelines for cost calculation [42].

\section{INCIDENCE OF INCISIONAL HERNIAS}

Incisional hernia after open surgery is a well-known complication of surgery, with an incidence of up to $20 \%$ after a 10 -year period [43]. In patients undergoing a sigmoid resection, Anderson et al. found that laparoscopic resection led to a significantly lower incidence of incisional hernia compared with open surgery [44]. Furthermore, in two retrospectively analyzed series of patients who received a partial hepatectomy, different types of incisions were compared. D'Angelica et al. reported that the common incisions used for partial hepatectomy were the Mercedes incision and extended right subcostal (ERSC) incision, and that the ERSC incision provides adequate, safe access and is associated with fewer long-term wound complications ( $9.8 \%$ vs $4.8 \%, \mathrm{P}<0.001$ ) [45]. More recently, Togo et al. reported frequencies of incisional hernia after median, J-shaped, right transverse incision with a vertical extension at the midline from the subumbilical region to the xiphoid process (RTVE), and reversed T incisions to be $6.3 \%, 4.7 \%, 5.4 \%$, and $21.7 \%$, respectively. A diagnosis of 'no hernia' required a minimum follow-up of 12 months [46].

To assess the incidence of incisional hernias in patients undergoing laparoscopic and open LLS, they will be contacted at a mean time of 1 year after resection to undergo ultrasonography to assess the incidence of incisional hernia.

\section{STUDY POPULATION}

Every patient requiring an LLS will be identified and informed at the outpatient clinic about open and laparoscopic liver resection. Only patients meeting the inclusion and exclusion criteria will be approached for randomization. After reading the ORANGE II trial patient information and being allowed 1 week for consideration, patients will be asked for their informed consent. All patients ineligible for randomization will be approached for participa-tion in the prospective registry. If patients express an explicit preference, they will be allocated to the prospective registry and interviewed to ascertain the reasons for their preferences. Personal written informed consent will be obtained for all groups. Randomization will be carried out through the ORANGE II trial website using web-based randomization software (TENALEA; www.tenalea. com) (see Figure 1 for trial flow-chart). 


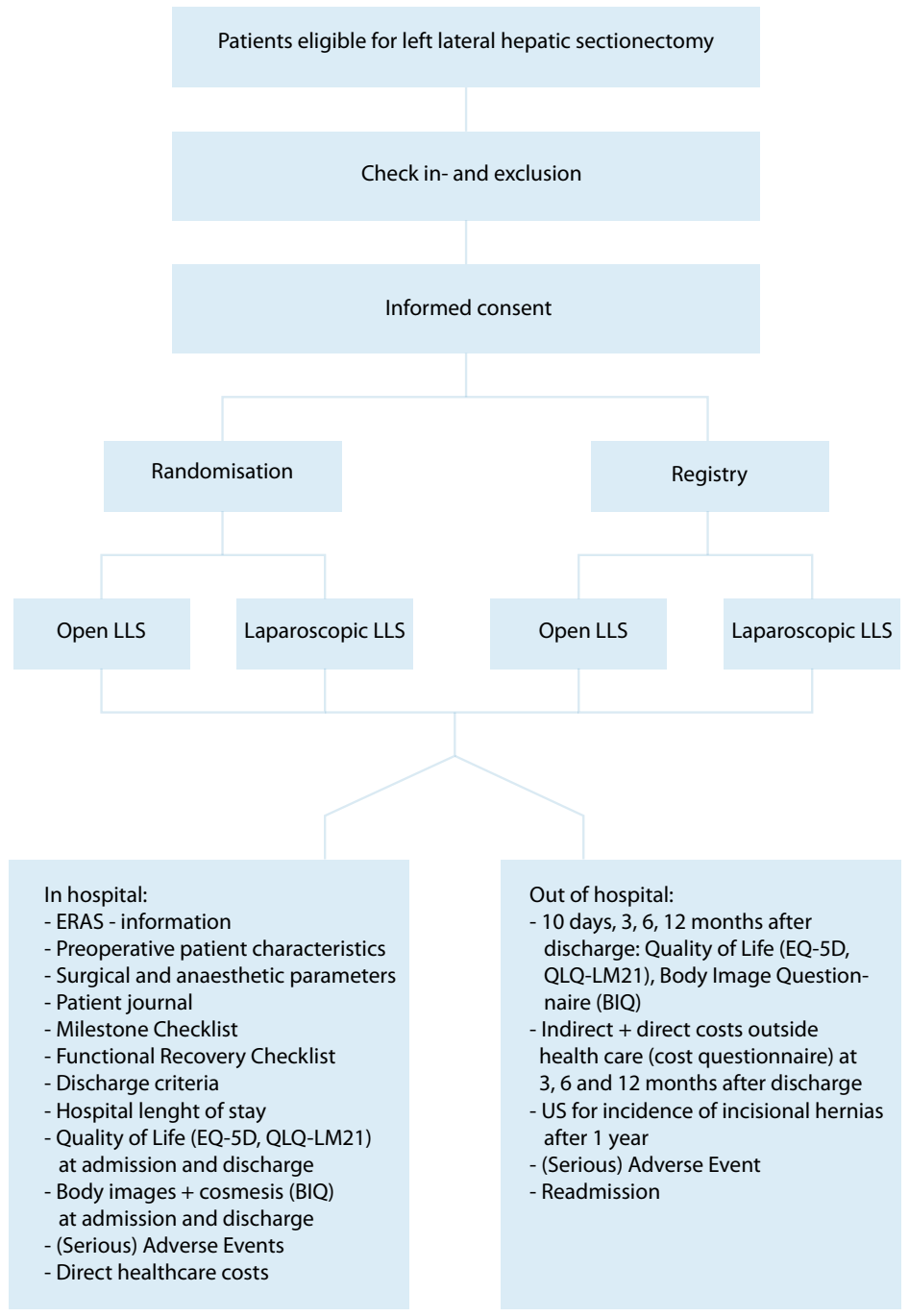

Figure 1 Orange II-Trial Flowchart.

Patients will be approached for randomized inclusion if they meet each of the following inclusion criteria: require LLS; willingness to participate in the study; able to understand the nature of the study and what will be required of them; are men or non-pregnant, non-lactating women between the ages of 18 and 80 years of age; have a body mass index of between 18 and 35; and have ASA grading of I to III. The exclusion criteria are: liver resection other than LLS; underlying liver disease; unwillingness to participate; inability to give written informed consent; and ASA grading of IVto V. 


\section{DAY BEFORE SURGERY:}

Normal oral nutrition up to 6 hours before surgery

No standard anxiolytic medication

Laboratory tests

\section{DAY OF SURGERY}

Carbohydrate drinks up to $2 \mathrm{hrs}$. prior to surgery

Balanced general anaesthesia + PCIA or PCEA

Preferably no nasogastric drainage, but when used

remove after surgery

Use warm i.v. fluids and upper and lower body

air-warming device

Avoid excessive i.v. fluids

CVP $<5 \mathrm{mmHg}$ during transection

Minimal incision / laparoscopy

No routine drainage of the peritoneal cavity

Patient sent to recovery ward

Restart oral intake of water / nutrition

\section{POST OPERATIVE DAY (POD) 1}

Patient sent to surgical ward

Patient mobilizes a minimum of four times a day

-sitting in chair / bedside

Discontinuation of i.v.-fluids

Patient drinks at least 1.5 litre

Normal diet

Continue PCIA or PCEA

1000 mg Aminocetophen 6 hourly
Start Laxatives

Laboratory tests

Check functional recovery criteria + discharge criteria

POD 2

Continue PCIA or PCEA

Stop low dose opioïds

Continue mobilization , $3 \times 1$ hour, walk

1000 mg Aminocetophen 6 hourly

Normal diet

Continue laxatives

Check functional recovery criteria + discharge criteria

\section{POD 3}

Stop / continue PCIA or PCEA

Remove urinary catheter (epidural)

Continue mobilization

Normal diet

Continue laxatives

Check functional recovery criteria + discharge criteria

\section{POD 4}

Check functional recovery criteria + discharge criteria

Discharge

Figure 2 Daily guideline of postoperative care of patients undergoing a hepatectomy in the ERAS programme.

\section{ERAS-PROGRAMME}

All patients will participate in the ERAS liver programme, with a standardized peri-operative management. For daily guidelines of the pre-and postoperative care of patients undergoing liver resection (Figure 2).

\section{Functional recovery criteria}

The evaluation of time to functional recovery will start on POD 0 and will be scored twice daily until discharge from the hospital. The discharge process starts at the pre-admission counseling session, during which any special needs of the patients will be determined (for 
example, homecare or social support, transport. Before admittance, any problem that could delay discharge will be identified and addressed. Patients will only be discharged when they have met the functional recovery criteria and are willing to go home. Reasons to delay discharge after functional recovery will be monitored and documented. Functional recovery criteria and LOS in hospital will be independently monitored and analyzed.

Criterion I: Normalizing serum bilirubin

Criterion II: Adequate pain control with oral analgesics

Postoperative pain will be systematically registered twice daily using the validated verbally administered 11-point numeric rating scale (NRS-11, 0 to 10) [47-50]. Members of a specialized pain team will ask patients to rate the intensity of their current pain on a scale of 0 (no pain) to 10 (worst possible pain), with pain rated as 'mild' (1 to 3) 'moderate' (4 to 6) or 'severe') 7 to 10 [51]. The NRS-11 seems to be better accepted by most patients and to be at least as sensitive and valid as the more traditional VAS ratings [50].

\section{Criterion III: Tolerance of solid food}

Fluid and solid food intake will be monitored and must return to normal, that is, when oral intake of water or normal food is resumed and continued for at least 24 hours. Furthermore the incidence of postoperative nausea and vomiting, which obviously influences intake, will be monitored postoperatively until day 6 using a scale ranging from 0 (no nausea) to 10 (worst possible nausea), and where necessary, be countered prophylactically by antiemetic treatment.

Criterion IV: No intra-venous fluids or medication administered

Criterion V: Independantly mobile or at preoperative level

To assess the difference between the preoperative and postoperative mobility level, the ERAS Mobility Scale (EMS) has been developed from the Groningen Activity Restriction Scale [52] The EMS assesses 10 basic actions to compare the level of mobility before and after surgical intervention. When the patient is able to perform 8 of the 10 items, they are independently mobile. Patients will be assessed whether they are able or not to independently perform these basic actions fully. Daily the assessment will be repeated and compared with the preoperative baseline score until mobility at an independent or pre-operative level is achieved.

\section{STATISTICAL ANALYSIS \\ SAMPLE SIZE}

Because laparoscopic liver surgery focuses on accelerated recovery, time to functional re- 
covery is used as the primary outcome parameter. Owing to the lack of hard evidence about the reduction in time to functional recovery after liver surgery, we have chosen to use the parameter that most accurately approaches our primary endpoint for our power calculation (LOS). Based on a retrospective analysis of 31 patients in both ERAS and non-ERAS settings, who have undergone LLS from 1990 to the present time, the mean \pm SD post-operative hospital stay for a LLS in the Maastricht University Medical Center is $6 \pm 2.73$. It therefore seems that that in patients undergoing laparoscopic LLS, time to functional recovery is reduced in comparison to patients undergoing the open procedure. We are aiming for a reduction in time to functional recovery of 2 days. A sample size of $2 \times 40$ patients in the randomization arms will be sufficient to show a 2-day reduction with a power of $90 \%$ and a level of significance at $a=0.05$ (two-tailed, given a within-arm SD of 2.73 with effect size $d=0.73$ ). Assuming an expected withdrawal rate of $\leq 10 \%$ during the trial, the participation of at least 10 centres, and the required addition of one randomized patient per arm for every additional participating centre $(C)$ to compensate for the loss of degrees of freedom incurred in the data analysis, which takes centre and treatment $x$ centre effects into account, a total sample size of $110(n=2 \cdot 55)$ will be required.

For all secondary outcome measures, the power will be $75 \%$ after correction for multiple testing with two-tailed $a=0.01$, assuming the same effect size $(d=0.73)$ as for the primary outcome. An interim analysis of the primary outcome, using Snapinn's method, will be performed after inclusion of $50 \%$ of the sample to avoid unnecessary inclusion of too many patients in this ORANGE II trial [53].

\section{DESCRIPTIVE STATISTICS}

The primary outcome parameter of time to functional recovery and the secondary parameter of LOS in hospital will be given in days, with a median and range. Morbidity will be classified according to the classification described by Dindo et al. and defined as a dichotomous composite endpoint, while readmission will be given as a percentage. Scores for quality of life, body image and cosmesis will be given as mean and standard deviation per time point per treatment arm. Hospital costs will be given as median and range. Long-term incidence of incisional hernia will be reported and analyzed.

\section{UNIVARIATE ANALYSIS}

The primary outcome measure of time to functional recovery will be measured in days, and will be analyzed with fixed-effect regression that will take centre and treatment $x$ centre interaction into account as fixed effects. If the actual number of centers and the sample size per centre allow random effects analysis, this will also be performed and this analysis will have the same power as the planned fixed effects analysis if the design effect does not exceed 1.2. With a sample size of 10 patients per centre, the design effect is 1.2 if the intra-class correlation (ICC) is 0.02 , where the ICC is based on treatment $x$ centre interaction [54]. All sec- 
ondary outcomes as measured at discharge will be analyzed by fixed-effect regression using linear regression for quantitative outcomes and logistic regression for binary outcomes, and including the baseline measure as a covariate to improve power and precision. In addition to P-values, confidence intervals for all effects will be reported. Morbidity will be classified as described by Dindo et al., but will be presented as raw data only because the required sample size for intervention effects on morbidity is much larger than the calculated sample size for this trial [55].

\section{ECONOMIC EVALUATION}

The economic evaluation will include a cost-utility analysis from a societal perspective. The time horizon of this evaluation will be the same as the duration of the trial, that is, 12 months. All costs (direct and indirect) related to both interventions will be calculated. The final cost calculation of unit costs will be based on a combined bottom-up and top-down approach. In accordance with Dutch guidelines for cost calculation, indirect healthcare costs will not be taken into account. In addition, resource use will be measured by use of primary data that is registered in our case record forms (CRFs) by use simple checklists. Furthermore, a questionnaire will be used to survey the direct non-healthcare costs related to travelling, impaired mobility and domiciliary care (for normal daily activities). The incremental, indirect non-healthcare costs per QALY gained will be based on the utility scores from the EQ-5D [36-38]. For all direct healthcare costs, the unit prices will be based either on prices from the hospital financial department or the Dutch guidelines for cost calculation [42].

\section{REGISTRY}

The prospective registry of patients who cannot be randomized because of ineligibility or because of explicit treatment preference on the part of the patient or surgeon will be analyzed as an observational study. In addition, data from the registry will be analyzed for interaction between treatment, centre, and study type (randomized or not). On condition that there is no interaction between treatment, centre, and study type, and that the observational study does not suffer from severe confounding (because adjusting for that strongly reduces the power of the observational study), pooling of both studies should give more power than separate analyses of either study. Possible confounders will be registered in the CRFs. The inclusion of the prospective registry in the trial design will create an uninterrupted case series, which will increase external validity and generalizability.

\section{DATA COLLECTION}

Data concerning patient characteristics, functional recovery, surgical and anaesthesiologic parameters, morbidity, LOS, QOL, patient compliance, and costs will be prospectively collected using both paper CRFs and an open source clinical trial software platform (OpenClinica; Ikaza Research, Cambridge, MA, USA) that uses e-CRFs for electronic data capture and clinical data management, which are validated and stored in compliance with good clinical 
practice guidelines. The e-CRFs will be stored in a secured database (Oracle Cor., Redwood Shores, CA, USA), and as stated previously, all patient data will be encoded to ensure privacy.

\section{MONITORING}

For this trial, a Data and Safety Monitoring Board (DSMB) has been appointed that will consist of three members: a chairperson, an independent statistician, and a medical specialist. In a concerted effort a DSMB charter will be developed, and all three members will sign a non-competing interest form. The DSMB will be responsible for safeguarding the interests of trial participants, assessing the safety and efficacy of the interventions during the trial, and monitoring the overall conduct of the clinical trial.

\section{INTENTION TO TREAT}

Analysis of all patients will be performed according to the intention-to-treat principle: patients will be analysed as randomised or as planned in the non-randomised prospective registry, and all patients will be included in the data analysis with proper methods for handling missing data.

\section{DISCUSSION}

Several authors have indicated that laparoscopic liver resection has many benefits over conventional open liver resection. However, this has never been proven in an RCT, and what the primary endpoint should be for an RCT comparing open and laparoscopic liver resection is a subject to debate. Using either liver surgery-related mortality or liver surgery-specific morbidity as an endpoint is not feasible, because patient accrual would take many years and be a logistically major global effort [55]. LOS in hospital, time to recovery, long-term incisional hernias, body image, and costs are potential candidates because improvements in these are some of the possible benefits. Laparoscopic liver resection is appealing for many surgeons and patients, but the learning curve for the surgeon is thought to be long and costly for hospital budgets. However, operating times in laparoscopic LLS tend to be shorter, and may compensate for expenses in technology and consumables [29, 56]. Moreover, the existing trials in liver surgery have not evaluated time to recovery or LOS in hospital after laparoscopic liver resection within an enhanced recovery programme. The more rapid recovery reported after enhanced recovery programmes may be further accelerated as a consequence of small incisions in laparoscopic surgery. In addition, learning curves for laparoscopic left lateral resection or anterior segments seem to be reasonably short for liver surgeons with advanced laparoscopic experience [57]. The question remains whether an RCT is necessary to prove that laparoscopy should be accepted as the preferred method to perform liver resection. In the Louisville consensus meeting on laparoscopic liver surgery, it was stated that laparoscopic LLS should be standard practice in experienced hands [58]. 
However, this may have been a subjective vision of a subset of opinion leaders, because long experience with both open and laparoscopic liver surgery was the main characteristic of those attending the meeting. Undoubtedly, the dissemination phase of laparoscopic liver surgery has started, and it is to be expected that many surgeons will adopt this technique in the future. A multinational multicentre prospective registry, a well organized multicentre $\mathrm{RCT}$, training programmes, and quality control measures are of great importance during this adoption period [31].

It is well recognized that a well-conducted double blind RCT provides the highest level of evidence to prove the possible benefits of laparoscopic liver resection. However, performing an RCT in surgery is not without difficulties, and alternative trial designs may be necessary $[31,32,59]$. First, the intervention needs to be tested in a standardized environment, and the properties of the intervention should remain unchanged during the trial period. This seems impossible for an intervention such as laparoscopic liver surgery in a multicentre RCT. Experience varies between participating centres, and will vary over time. Moreover, local standards for perioperative care are different. Both LLS and the ERAS enhanced recovery protocol provide the standardization needed. The learning curve of a LLS is short in centres with experience in liver surgery and advanced laparoscopy. The use of proctor surgeons in centres with limited experience in laparoscopic liver surgery the operative techniques can be reasonably standardised, and this should eliminate learning curve influences on outcome parameters. Quality of the surgery can be assured by digital video recording.

Second, the intervention should be double-blinded. Although double blinding in a surgical trial is difficult, using a fixed abdominal dressing for 3 days after surgery is feasible, and should prevent both ward caregivers and patients from knowing the type of intervention. Third, it is reasonable to query whether this is now the right time to perform an RCT and whether the results of the trial will be valid for the more general surgical community. A recent review of the results of laparoscopic liver resection in 2,804 patients showed that laparoscopic liver resection in expert centres is feasible and safe for both minor and major liver resections [16]. The percentages of liver resections performed laparoscopically now range from $25 \%$ to $65 \%$ in high-volume expert centres such as University Hospital Southampton NHS (Southampton, UK), Henri Mondor (Paris, France), UPMC (Pittsburgh, USA), UZ Leuven (Leuven, Belgium) and Rikshospitalet (Oslo, Norway) [16,60]. Although it is to be expected that many centres worldwide will adopt laparoscopic liver resection as a more or less standard procedure in the near future, there are still many patients and surgeons that prefer the open procedure long beyond the learning curve. In parallel with the development of laparoscopic liver surgery, 'fast-track' programmes in various areas of surgery, including liver surgery, are gaining popularity. Therefore, this seems to be the right time for this RCT to be performed. The multicentre character of the ORANGE II trial with randomization of patients and surgeons with treatment equipoise and a prospective registry to cover both surgeons 
who believe that based on their laparoscopic experience randomization is not ethically justified and patients with a strong treatment preference will provide external validity. This trial design capitalizes on rather than ignores the differences between patients, will provide more robust outcome data, and should lead to continuous performance monitoring after the trial $[33,59]$.

The key question clearly is as to whether this RCT is really necessary. The benefits of laparoscopic liver resection are not beyond reasonable doubt, and although data are becoming increasingly available, recent publications do not provide sound data on time to recovery. Worldwide, median LOS in hospital for open and laparoscopic resections varies from 4 to 8 days [17, 28, 61-63]. Reasons for delay in discharge and discharge location are often absent, and a clear definition of recovery has not been used to date in any of the publications. Departing from the standpoint that an RCT should be conducted, the question is which sample size should be used? In our opinion, a reduction of only 1 day in time to recovery or LOS in hospital after laparoscopic resection would be a disappointingly low gain. To prove such a reduction, 320 patients would be needed ( $\alpha=0.05$ and power of $90 \%$ ), making the trial unlikely to be accomplished. Based on available reports, a 2-day reduction should be possible, and reduces the sample size to 110 patients undergoing LLS[17, 29,63]. This number is reasonably moderate, and it is to be expected that patient accrual will be accomplished within 1-2 years.

It should be realised that many centres have introduced laparoscopic liver surgery programmes in the absence of a central reporting or certifying agency. In our opinion, laparoscopic LLS should function as a model for further dissemination of laparoscopic techniques in hepatic surgery. The left lateral segment of the liver has been a natural first step for a laparoscopic resection given the peripheral anatomical location (thin liver segment, minimal requirement for biliary dissection, and ease of controlling the left portal pedicles and left hepatic vein), and has been proven to be safe and feasible with reproducible results $[3,16]$. The implementation of the laparoscopic LLS may not only serve as a guide to develop and master programmes for major laparoscopic hepatic resections, but may also be used as an introduction for centres new to laparoscopic approaches in liver surgery. To adopt laparoscopic liver resection safely, certification for centres, surgeons, and units should be available through the International Hepatobiliary (HPB) Association, and national and international HPB associations should become involved in the goal of establishing training standards and credentials to ensure a high and consistent outcome. The ORANGE II trial in which techniques are standardised and a training and proctor programme is available, combined with the hybrid design of randomisation and registry may help to provide a framework for controlled and safe implementation of laparoscopic liver resection across participating centres. 


\section{CONCLUSION}

The international multicentre randomised controlled ORANGE II trial is based on the observations of more rapid recovery and discharge after laparoscopic liver resection, and more rapid recovery and discharge after open liver resection within an enhanced recovery programme. This is the first RCT to provide evidence on the merits of laparoscopic surgery in patients undergoing a hepatic left lateral sectionectomy 


\section{ABSTRACT - ORANGE II PLUS TRIAL}

\section{BACKGROUND}

Recent developments in liver surgery include the introduction of laparoscopic surgery and enhanced recovery programmes. Laparoscopic surgery and enhanced recovery programmes both focus on faster time to recovery and consequently shorter hospital length of stay. The added value of the laparoscopic hemihepatectomy compared to the open hemihepatectomy in an ERAS setting has never been studied in a randomised controlled setting. The multicentre international ORANGE II PLUS - Trial will provide evidence on the merits of laparoscopic compared with open hemihepatectomy.

\section{METHODS/DESIGN}

Patients eligible for left or right hemihepatectomy will be recruited and randomised at the outpatient clinic. All randomised patients will be operated in the setting of an ERAS programme. The experimental design produces two randomised arms (open and laparoscopic hemihepatectomy) and a prospective registry. Patients ineligible for randomization can be included in the prospective registry. The primary endpoint of the ORANGE II PLUS trial is time to functional recovery. Secondary endpoints are hospital length of stay, intraoperative blood loss, operation time, resection margin, time to adjuvant chemotherapy initiation, readmission percentage, (liver specific) morbidity, quality of life, body image, reasons for delay of discharge after functional recovery, long term incidence of incisional hernias, hospital and societal costs during one year, overall five-year survival. We aim at a reduction in time to functional recovery by 2 days after laparoscopic hemihepatectomy. A sample size of 125 patients in each randomisation arm has been calculated to detect a 2-day reduction in time to functional recovery (power $80 \%$ and $a=0.04$ two-tailed in the final analysis, to adjust for interim analysis with $\mathrm{a}=0.01$ halfway the trial).

\section{CONCLUSION}

The ORANGE II PLUS trial is a multicentre RCT that will provide evidence on the merits of laparoscopic surgery in patients undergoing hemihepatectomy within an enhanced recovery ERASprogramme.

ORANGE II PLUS TRIAL REGISTRATION: Clinicaltrials.gov NCT 01441856 


\section{INTRODUCTION}

Liver resection for colorectal metastasis is the only potentially curative therapy and has become the standard of care in appropriately staged patients, offering 5-year survival rates of approximately $35-40 \%[1]$. Also for symptomatic benign lesions and those of uncertain nature or large size, liver resection is a widely accepted treatment. Open hepatectomy $(\mathrm{OH})$ is the current standard of care for the management of primary and secondary malignancies. Although the feasibility of laparoscopic hepatectomy (LH) has been established, only select centres have used this technique as their primary modality[64].

Laparoscopic liver resection was first reported in 1991[2, 65-68]. Over the last decade the method has gained wide acceptance for various liver resection procedures[3-5]. Multiple retrospective case series and reviews comparing open with laparoscopic liver resection indicate that laparoscopic liver resection can be applied safely for both malignant and benign liver lesions[10-13, 15]. Laparoscopic liver resection has been associated with shorter hospital length of stay, reduced intraoperative blood loss, less postoperative pain, earlier recovery and better quality of life[9, 13, 18, 19]. Initially the left lateral segments of the liver were chosen for anatomic laparoscopic resection with good results. Many liver centres worldwide are currently adopting laparoscopic surgery for resection of anterior segments. A relatively low volume, a significant learning curve and lack of evidence restrict the majority of liver surgeons to further adopt and disseminate this technique.

A new impulse for the laparoscopic management of liver lesions came after the first reports of laparoscopic hemihepatectomies[69-71]. Major hepatic resections are technically demanding, time consuming and are thought to hold an increased risk for morbidity. It was demonstrated that in expert hands major anatomical laparoscopic liver resections were feasible with good efficacy and safety. Expert liver centres are performing laparoscopic (extended) hemihepatectomies, but doubt remains on clinical efficacy and societal costs. Currently, in European centres a median hospital length of stay of 6.0 to 13.1 and 3.5 to 10.0 days is observed after open and laparoscopic hepatic resection respectively[72]. In expert hands median duration of admission after major hepatic resection varies between $6-12.5$ for open and $4-8.2$ for laparoscopic surgery[7, 8, 71, 73-75]. However, reports are scarce and level-1 evidence on this matter is still to be presented[7, 73, 74, 76].

Within the framework of optimizing postoperative recovery, broader indications for hepatic resection and further adoption of laparoscopic liver surgery there is a need for a randomized trial. Regarding postoperative care, enthusiasm has recently arisen for the Enhanced Recovery After Surgery (ERAS) programme. This multimodal programme, derived from Kehlet's 1990's pioneer work in the multimodal surgical care field, involves optimization of several aspects of the perioperative management of patients undergoing major abdominal surgery 
(Appendix 1). In liver surgery it was demonstrated that hospital length of stay after open and laparoscopic liver resection could be reduced when patients were managed within a multimodal ERAS programme[28, 29]. In multiple studies there was a discrepancy between hospital length of stay (LOS) and functional recovery[30]. This delay is often linked to social problems, problems in homecare support or logistic problems.

The added value of the laparoscopic hemihepatectomy compared to the open hemihepatectomy has never been studied in a randomized controlled setting. Therefore, the multicentre international ORANGE II PLUS - trial has been constructed and will provide evidence on the merits of laparoscopic versus open hemihepatectomy in terms of time to functional recovery, hospital length of stay, intraoperative blood loss, operation time, resection margin, time to adjuvant chemotherapy initiation, readmission percentage, (liver specific) morbidity, quality of life, body image, reasons for delay of discharge after functional recovery, long term incidence of incisional hernias, hospital and societal costs during one year and overall five-year survival.

\section{METHODS AND DESIGN}

\section{STUDY DESIGN}

The ORANGE II PLUS - trial is a prospective study with a two armed, multicentre randomised double blinded experimental design and registry to determine whether laparoscopic surgery is to be preferred over open surgery in patients undergoing a hemihepatectomy. Patients, and if possible also the medical and nursing staff, will be blinded for the type of intervention using an abdominal dressing until postoperative day 4 . Follow-up will take place at respectively 10 days, 3, 6, 12 months and 5-years after discharge.

\section{PRIMARY \& SECONDARY ENDPOINTS}

The primary endpoint of the ORANGE II PLUS - Trial is time to functional recovery. A patient is fully functionally recovered when all of the five criteria are satisfied. These criteria are clearly defined as follows:

- $\quad$ Adequate pain control with oral analgesics;

- Independently mobile

- $\quad$ Tolerance of solid food;

- $\quad$ Normal or decreasing TB (total bilirubin), ALT, AST, and INR normal or at least $80 \%$ of the normal value.

- $\quad$ No intravenous fluids

When all of these five criteria are met, we consider a patient functional recovered. It is medically justified to discharge patients when the criteria for full functional recovery are met and 
if the patient is willing to go home. Secondary endpoints include hospital length of stay, intraoperative blood loss, operation time, resection margin, time to adjuvant chemotherapy initiation, readmission percentage, (liver specific) morbidity, quality of life, body image, reasons for delay of discharge after functional recovery, long term incidence of incisional hernias, hospital and societal costs during one year and overall five-year survival.

\section{TIMETO FUNCTIONAL RECOVERY}

The evaluation of time to functional recovery will start on POD 0 and will be scored twice daily until discharge from the hospital. The discharge process starts at the pre-admission counseling session where any special needs of the patients will be determined (e.g. homecare support, transport, social support). Before admittance, any problem that could delay discharge will be identified and addressed. Patients will only be discharged when they have met the functional recovery criteria and are willing to go home. Reasons to delay discharge after functional recovery will be monitored and documented. Functional recovery criteria and hospital length of stay are independently monitored and analyzed.

\section{Adequate pain control with oral analgesia}

Postoperative pain will be systematically registered twice daily using the validated verbally administered 0-10 NRS-11 - 11 points numeric rating scale[47-49]. Members of a specialized pain team ask patients to rate the intensity of their current pain on a scale of 0 - "no pain" to 10 - "worst possible pain". A score of 1-3 will be considered to be mild, 4 to 6 to be moderate and 7 to 10 to be severe[47].

\section{Tolerance of solid food}

Fluid and solid food intake will be monitored and must be returned to normal tolerance level. Tolerance is considered to be normal when oral intake of water or normal food is resumed and continued for at least 24 hours. Furthermore the postoperative nausea and vomiting (PONV), which obviously influences the intake, will prophylactic be countered (antiemetic) and postoperatively also be registered, until the 6th day after surgery, by use of a scale ranging from 0 - "no nausea" to 10 - "worst possible nausea".

\section{Mobile at preoperative level}

In order to report the difference between pre- and postoperative level of mobility, the 'ERAS Mobility Scale' (EMS) (appendix 1), which is inferred from the 'Groningen activity restriction scale, was developed[52]. The EMS utilizes ten items of basic actions to compare the level of mobility before and after surgical intervention. When the patient is able to perform 8 out of 10 items, they are independently mobile. Patients will have to report whether they are able 
to fully independently perform these basic actions or not. Twice a day the assessment will be repeated and compared to the preoperative baseline score until mobility at an independent level is achieved.

\section{MORBIDITY}

Patients' preoperative morbidity status is measured using the ASA-scoring. The Satava classification (appendix 6) adapted for liver surgery will be used for intraoperative unfavourable incidents[77, 78]. Intraoperative blood loss will be separately assessed as a secondary endpoint. Postoperative morbidity is rationally predictable, hemorrhagic complications occurring predominantly during surgery or in the early postoperative phase, and biliary complications, intra-abdominal abscess or liver failure in the later postoperative phase. Also wound infection and sepsis will be complications to monitor. Morbidity will be classified and analyzed according to the recently reported Accordion Severity Grading System of Surgical Complications as described by Strasberg et al.[79, 80].

\section{LIVER RESECTION SPECIFIC COMPOSITE ENDPOINT}

In this trial we have chosen to use a well defined liver surgery-specific composite endpoint, as suggested by van den Broek et al.[35].[41] This endpoint is a parameter composed of a combination of procedure-specific complications and considered as a single, dichotomous outcome: operative mortality, intra-abdominal haemorrhage, ascites, bile leakage, intra- abdominal abscess and postresectional liver failure. These components, which are all specific to liver surgery and have substantial clinical relevance, reflect Dindo grade 3-5 complications.

\section{QUALITY OF LIFE}

To assess quality of life in patients undergoing laparoscopic hemihepatectomy versus those undergoing open hemihepatectomy, the Dutch version of the EQ-5D (EuroQol Group) status test in Dutch centres and the translated EQ-5D for international centres will be used. EQ$5 \mathrm{D}$ (appendix 2) is a standardized instrument for use as a measure of health outcome, which consists of five dimensions (mobility, self-care, usual activities, pain/ discomfort and anxiety/ depression) with three levels each and an EQ-VAS score (0-100)[36-38]. Furthermore, the EORTC QLQ-C30 (appendix 3) with the LM21 module will be used for liver specific treatment measurements[39]. Assessment of the patients' quality of life will be performed at the time of consent, discharge and 10 days, 3, 6 and 12 months after discharge.

\section{BODY IMAGE AND COSMESIS}

In order to evaluate differences in postoperative body image and cosmesis, the Body image Questionnaire (BIQ) will be used (appendix 4)[40,41]. The BIQ consists of 8 questions regarding body image and cosmesis. The body image assessment will be performed preoperatively at time of consent. Both the body image and the cosmesis assessment will take 
place at discharge, 10 days, 3, 6 and 12 months after discharge.

\section{LONG-TERM INCIDENCE OF INCISIONAL HERNIAS}

Incisional hernia after open surgery is a well-known complication with an incidence of up to $20 \%$ after a 10-year period[43]. In patients undergoing a sigmoid resection, Anderson et al. found that laparoscopic resection leads to a significantly lower incidence of incisional hernia compared with the open surgical technique[44]. Furthermore, an incidence of incisional hernia of $9.2 \%$ after an open liver resection is reported by lto et al. and a recent RCT by Halm et al. comparing transversal versus midline incision for upper abdominal surgery demonstrated an incidence of respectively $1.7 \%$ and $14.5 \%[63,81]$. To assess the incidence of incisional hernias in laparoscopic and open hemihepatectomies patients will be contacted at a mean time of 1 year after resection to receive an ultrasound to diagnose incisional hernia.

\section{ECONOMIC EVALUATION}

\section{(In)direct treatment costs}

These expenses constitute of personnel and material costs. Total operating time, hospital days, operating theater and material costs (e.g. disposables, monitors, endoscopic tower), personnel costs (surgeon, anesthesiologist, assistants, operating nurses) will be documented. Intraoperative costs will be obtained from operating nurses who will register the use of all instruments and other items. Unit prices will be based on prices from the participating hospital financial departments or, if unavailable, will be based on general national guidelines for pricing. Pre-admission charges for screening will not be taken into account, as these are equal for both surgical techniques. All readmission related costs will be added to the total hospital expenses. Furthermore, postoperative outpatient clinic visits, general practitioner consultations, and home care related costs will be documented and quantified in the CRF. A cost questionnaire offered at the regular follow-up consultation (3, 6 and 12 months) will help us to assess the societal and individual costs outside health care relating to patients'absence, impaired mobility, work or normal daily activities.

\section{Societal Costs and Cost-Utility Analysis}

For patients performing paid labour productivity loss will be calculated using the humancapital approach, which counts any hour not worked as an hour lost. Preoperative assessment will document the occupation and the full- or part-time appointment. The incremental costs per Quality Adjusted Life Year (QALY) gained will be based on utility scores from the EQ-5D combined with the total (direct + indirect) patient costs[36-38]. The Quality of Life forms will be offered simultaneously with the cost questionnaires during regular follow-up.

\section{SURGERY}

The intraoperative surgical technique in this study will not be standardized. Surgeons in the participating centres, that perform the open or laparoscopic hemihepatectomy, are free to 
use their preferred technique and devices to gain intra-abdominal access, perform parenchymal transection and maintain vascular control. Total operation time and intraoperative blood loss will be monitored as secondary outcome parameters.

\section{LABORATORY}

Preoperatively, venous blood samples will be drawn and analysed for blood group (rhesus factor, irregular antibodies), haemoglobin, leucocytes, platelets, PT, aPTT, INR, kidney function (Sodium, Potassium, Urea, Creatinin), liver function (AF, Y-GT, AST, ALT, LDH, Bilirubin), CRP, CEA and AFP. In the postoperative days venous blood samples will be drawn on POD 1-2-3-5 and POD 7 (additional testing can be considered if indicated). Postoperative blood tests will be almost identical to the preoperative tests.

\section{PATHOLOGY}

Resection margin will be defined in both millimeters and status (R0 - R2) in all resected specimens as a secondary endpoint. The resected non-tumorous liver parenchyma will be analyzed by an experienced pathologists in each participating centre for underlying liver disease. Level of fibrosis, inflammation and steatosis will be determined. The METAVIR score will reflect fibrosis (F0-F4) and inflammatory activity (A0-A3)[82].[52] In addition, the level of steatosis (Grade 0 to 3 ) and presence of chemotherapy-induced hepatopathy (Sinusoidal Obstruction Syndrome = SOS, Chemotherapy-Associated Steatohepatitis = CASH) will be defined. This will enable stratification for underlying liver disease during data analysis.

\section{ONCOLOGICAL ENDPOINTS}

Not only will the resection margin be defined in pathological examination. During follow-up we also aim to monitor the time frame between the liver resection and the start of adjuvant chemotherapy (if indicated) and the 5-year overall survival.

\section{STUDY POPULATION}

The research population will be drawn from the outpatient clinic of the departments of surgery. In all patients with the need for hemihepatectomy, the inclusion criteria will be tested. Every patient fulfilling the inclusion criteria will be recruited for randomization. Here, after 1 week of reflection, written informed consent will be obtained at the ERAS-information conversation. All patients ineligible for randomization will be approached for participation in the prospective registry. All reasons for non-randomized inclusion will be registered. Personal written informed consent will be obtained in all groups. Randomization will be done through the ORANGE II PLUS trial website by web based randomisation software (TENALEA ${ }^{\oplus}$, online randomisation module). See Figure 1 for trial flow-chart.

\section{INCLUSION CRITERIA}

Patients requiring open or laparoscopic left / right hemihepatectomy for accepted indica- 
tions; able to understand the nature of the study and what will be required of them; men and non-pregnant, non-lactating women between age 18-80; BMI between 18-35; patients with ASA I-II-III.

\section{EXCLUSION CRITERIA}

Inability to give written informed consent; patients undergoing liver resection other than left or right hemihepatectomy; patients with hepatic lesion(s), that are located with insufficient margin from vascular or biliary structures to be operated laparoscopically; patients with ASA IV-V; repeat hepatectomy.

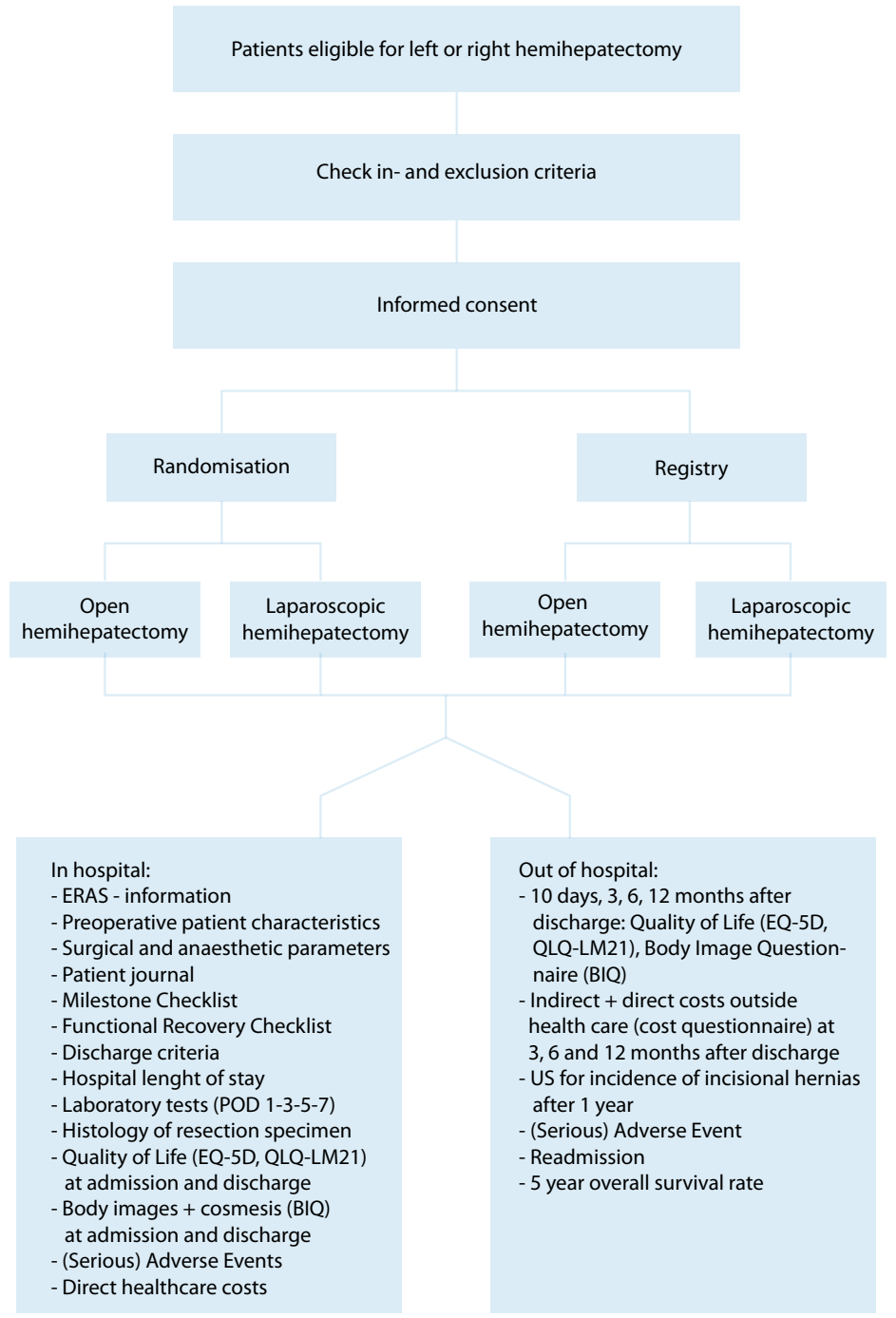

Figure 1. ORANGE II PLUS - Flowchart 


\section{DAY BEFORE SURGERY:}

Normal oral nutrition up to 6 hours before surgery

No standard anxiolytic medication

Laboratory tests

\section{DAY OF SURGERY}

Carbohydrate drinks up to $2 \mathrm{hrs}$. prior to surgery

Balanced general anaesthesia + PCIA or PCEA

Preferably no nasogastric drainage, but when used remove after surgery

Use warm i.v. fluids and upper and lower body air-warming device

Avoid excessive i.v. fluids

CVP $<5 \mathrm{mmHg}$ during transection

Minimal incision / laparoscopy

No routine drainage of the peritoneal cavity

Patient sent to recovery ward

Restart oral intake of water / nutrition

\section{POST OPERATIVE DAY (POD) 1}

Patient sent to surgical ward

Patient mobilizes a minimum of four times a day

-sitting in chair / bedside

Discontinuation of i.v.-fluids

Patient drinks at least 1.5 litre

Normal diet

Continue PCIA or PCEA

1000 mg Aminocetophen 6 hourly
Start Laxatives

Laboratory tests

Check functional recovery criteria + discharge criteria

POD 2

Continue PCIA or PCEA

Stop low dose opioïds

Continue mobilization , $3 \times 1$ hour, walk

1000 mg Aminocetophen 6 hourly

Normal diet

Continue laxatives

Check functional recovery criteria + discharge criteria

\section{POD 3}

Stop / continue PCIA or PCEA

Remove urinary catheter (epidural)

Continue mobilization

Normal diet

Continue laxatives

Check functional recovery criteria + discharge criteria

\section{POD 4}

Check functional recovery criteria + discharge criteria

Discharge

Figure 2. Daily guidelines of care for patients undergoing hepatectomy in the ERAS programme.

\section{ERAS-PROGRAMME}

All patients will participate in the ERAS-liver programme providing a standardised perioperative management. For daily guidelines of the pre- and postoperative care of patients undergoing liver resection in the ERAS programme, see Figure 2. 


\section{ETHICS}

The study has been approved by the Medical Ethical Review Board of the Maastricht University Medical Centre, Maastricht, The Netherlands trial number NL36215.068.11 / MEC 11-2028.

Ethical consent will also be obtained from national or regional ethical boards in each participating country. Patients willing to participate in this trial will receive both verbal and written information at the time of recruitment in the outpatient clinic. In accordance with the local medical ethics committee all participating sites will provide an independent surgeon / physician if needed. An independent surgeon (Dr. M. Poeze) has been appointed for the Maastricht University Medical Centre to answer questions. Confidentiality is guaranteed by assigning the participators an encoded trial number. This indicates that only the physician with the decoding 'key' knows which procedure is assigned. All trial data will be saved during the trial and stored on a secured data-server. In addition, the patient's will be asked to consent to future analysis of these data. Withdrawal from the trial at any time or for any reason will not hold any form of consequences for the patient.

\section{STATISTICAL ANALYSIS}

\section{SAMPLE SIZE}

Since laparoscopic liver surgery focuses on accelerated recovery, time to functional recovery is used as primary outcome parameter. In the largest combined prospectively collected database of three European, two U.S., and one Australian centre a median hospital length of stay of 6.0 days (SD: 4.5) was observed after major laparoscopic hepatic resection. In European centres the median LOS was significantly longer compared to the non-European hospitals (8.5 vs 5.9 days; $P=0.0001$ )[7]. In a recent single centre report, of which data were also used in the aforementioned database analyses, 22 laparoscopic and 50 open major liver resections were evaluated. They demonstrated a mean hospital length of stay of 8.2 days (SEM $1.1, \mathrm{SD}=5.1$ ) after laparoscopic hepatectomy[6]. A large analysis of Jarnagin et al. showed a mean duration of admission after open major liver resection of 11.1 days[83]. Based on these reports an average reduction in hospital length of stay of 2 days seems feasible and LOS mean and SD after a laparoscopic hemihepatectomy in Europe can be estimated to be 8.5 and 5.0 days, respectively. We assume that in patients undergoing laparoscopic left or right hemihepatectomy, time to functional recovery is reduced in comparison to patients undergoing open procedure. We aim at a reduction in time to functional recovery of 2 days. Assuming an SD of 5.0 for time to functional recovery, which implies an effect size of $d=0.40$, and taking into account a drop- out rate of $10 \%$, a loss in degrees of freedom for estimating covariate effects (centre, left/right hepatectomy), a total sample size of 250 patients ( $125 \mathrm{per}$ arm) can demonstrate a 2 day reduction with a level of significance $a=0.04$ two-tailed and a power of $80 \%$. An interim analysis of the primary outcome (with $a=0.01$ two-tailed) will be performed after inclusion of $50 \%$ of the sample. 


\section{DESCRIPTIVE STATISTICS}

Primary outcome parameter time to functional recovery and secondary parameter hospital length of stay are given in days (mean, median, SD, range). Morbidity will be classified according to the classification described by Dindo et al. and defined as a dichotomous composite endpoint, readmission percentage will be given as mean and standard deviation. All other outcomes and baseline characteristics will be summarized by descriptive statistics per treatment arm of the RCT. This procedure will be repeated for the non-randomised study part, but now with significance tests of baseline group differences to detect the most important candidate confounders.

\section{INFERENTIAL ANALYSIS}

The primary outcome measure "time to functional recovery" will be measured in days and will be analyzed with fixed effects linear regression, taking into account treatment, centre, left versus right hepatectomy, patient age and gender, and using $a=0.04$ two-tailed for the treatment effect in view of the interim analysis. All other outcomes will be similary analysed with regression (linear for quantitative outcomes, logistic for binary outcomes), but now with $a=0.01$ two-tailed to adjust for multiple testing. Repeatedly measured outcomes will be analysed with mixed regression for repeated measures in order to include persons with missing data. Morbidity will be classified following Dindo et al. and will only be presented as raw data since the required sample size for intervention effects on morbidity is much larger than the calculated sample size for this trial[55]. Treatment by covariate interactions will be explored and if found, will lead to subgroup analyses. These are secondary analyses, however, in view of the lower power for interaction effects and the increased risk of type I errors due to multiple testing. Finally, all analyses will be repeated for the non-randomized part of the study and the results will be compared with those from the RCT.

\section{INTENTION TO TREAT}

Analysis of all patients will be done according to the intention to treat principle: patients will be analyzed as randomized. Thereby, a small number of patients that require an unplanned hand-assisted procedure or another liver resections based on intraoperative evaluation will also be included in the analysis. In addition, whenever the blinding cannot be continued until POD 4, patients will be analyzed as intention-to-treat. All patients will be included into data analysis with proper methods for handling missing data. 


\section{INTERIM ANALYSIS}

An interim analysis of the primary outcome and of mortality will be performed after inclusion of $50 \%$ of the sample, using two stopping rules:

\section{1) Stopping for significance}

The trial will be stopped if an interim analysis after $50 \%$ of the needed randomized sample size $(\mathrm{N}=125)$ shows a significant difference between both trial arms with respect to the primary outcome, time to functional recovery, at a two-tailed alpha of 0.01 . Else, the trial will be continued.

\section{2) Stopping for safety}

The trial will be stopped if the mortality after hemihepatectomy in patients with a normal liver is greater than $5 \%$ or is greater than $10 \%$ in cirrhotic patients at interim analysis after $50 \%$ of the needed randomized sample size $(\mathrm{N}=125)$ has been included (irrespective of the trial arm).

\section{DATA COLLECTION}

All patient data will be prospectively collected using both paper CRFs and OpenClinica ${ }^{\circledR}$ (Ikaza Research, Cambridge, Massachusets, USA) and stored in a secured Oracle database (Oracle Corporation, Redwood Shores, CA, USA). OpenClinica is an open source clinical trial software platform, that uses e-CRF's, for electronic data capture (EDC) and clinical data management (CDM), validated and stored in compliance with good clinical practice (GCP) guidelines. All patient data will be encoded to ensure the patient's privacy.

\section{DATA SAFETY MONITORING BOARD (DSMB)}

For this trial a DSMB has been appointed that consists of at three members: a Chair, a independent statistician and a medical specialist. In a concerted effort a DSMB - charter has bee developed and all three members have signed a non-competing interest form. The charge to the DSMB is to safeguard the interests of trial participants, assess the safety and efficacy of the interventions during the trial, and monitor the overall conduct of the clinical trial. 


\section{DISCUSSION}

Optimization of the patient's recovery is what both minimally invasive liver surgery and multimodal enhanced recovery programmes aim to achieve. Laparoscopic liver resection has many suggested benefits over conventional open liver resection, such as less pain and analgesic requirements, smaller scars with preservation of the abdominal wall, shorter hospital length of stay, less transfusion requirements, faster recovery, and less postoperative adhesions[16]. High-volume expert centres in the United Kingdom, France, Norway and USA now perform up to $60 \%$ of their liver resections laparoscopically[16, 60]. Centres with less experience in the laparoscopic approach plan to adopt or specialize in this technique. This is attractive, but the laparoscopic technique also has a considerable learning curve and seems more expensive[84].

Developments in liver surgery not only focus on laparoscopic surgery, but also aim for provision of optimal perioperative care. A standardized perioperative care protocol, such as the Enhanced Recovery After Surgery (ERAS) programme, has proven to reduce time to recovery and length of stay[28]. Moreover, a laparoscopic approach combined with an ERAS programme may have a synergistic effect as suggested in a small pilot trial in liver surgery and a RCT in colonic surgery[36, 85].

A review of 2,804 patients undergoing laparoscopic liver resection found that the technique is feasible and safe[16]. A recent review by Lin et al. concluded that laparoscopic techniques are successfully used for major hepatectomy in selected patients, but that further studies would be helpful to identify the clinical value concerning patient and economic outcomes, major hepatectomy-specific and long-term oncologic outcomes in particular[86]. A group of renowned experts feel that the benefits of laparoscopic minor liver surgery, left lateral sectionectomies in particular, are beyond doubt[7].

The feasibility and necessity of an RCT investigating laparoscopic minor liver resections in the anterior segments is questioned by these experts[58]. However, the ORANGE II trial comparing open and laparoscopic left lateral segmentectomy within an ERAS programme is accruing patients since 2010 and a worldwide survey among liver surgeons showed that a trial was still warranted[87, 88]. Operating times in laparoscopic minor liver surgery have been suggested to be reduced and this may compensate for increased expenses[29, 56]. This proposed advantage has not been seen in major liver resection and most experts still have doubts on clinical efficacy and societal costs. Hence, there is actually no debate on the necessity of a randomized trial comparing laparoscopic versus open major liver resection. This is also reflected in the Louisville Statement of 2008[58]. This expert consensus proposed that, although laparoscopic major liver resections have been shown to be feasible and safe, they should be reserved to experienced surgeons already comfortable with more limited laparoscopic liver resections[58]. 
While expert centres are already performing laparoscopic hemihepatectomies, there is a need for prospective a randomised trial within the framework of optimising postoperative recovery, broader indications for resection and further adoption of major laparoscopic liver surgery. Also credentialing remains a challenging issue, as lack of consistent training is often the reality[89]. The debate among experts does not solely focus on the necessity of an RCT, but also on what endpoint(s) should be used. A trial with liver-surgery-related mortality or morbidity as the primary endpoint may lead to nearly infeasible patient accrual due to large sample sizes[55]. Hospital length of stay, time to recovery, long term incisional hernias, cosmesis and costs are better alternatives, but surgeons also desire prospective evidence on oncological outcomes, such as radicality of resection, time needed to first adjuvant chemotherapy and overall or disease free survival, quality of life and intraoperative parameters such as blood loss. Length of stay is however an endpoint that is hard to compare between centres as there is a gap between the moment a patient is functionally recovered and the actual discharge[30,90]. This present study will not only address the clinically relevant primary outcome "time to functional recovery", it will also assess important intra- and postoperative outcomes including intraoperative complications, a composite endpoint of liver-specific morbidity, oncological outcomes, quality of life, pain and costs. A randomized controlled trial will give the highest level of evidence, but it may be difficult to conduct in the surgical community. A currently ongoing trial comparing minor liver resections suffers from slow accrual due to the low incidence of LLS and both patient and surgeon preference for laparoscopic liver resection[87]. Not only does the intervention need to be standardized, also the environment in which the intervention is studied needs to be controlled. Although the experience with laparoscopic hemihepatectomy may vary between participating centres, all participating centres will be experienced and high-volume centres[91]. The distinct choices to only include hemihepatectomies and to use an ERAS programme provide the standardization needed. Although blinding with a fixed abdominal dressing for 4 days is difficult and will not always be feasible for the ward staff, the intention of this ORANGE II Plus - Trial is to double blind the intervention (patient and ward staff) to eliminate bias in primary outcome. An additional prospective registration of patients ineligible for or refusing randomization will strengthen external validity. 


\section{CONCLUSION}

To optimise perioperative care and to reduce time to recovery minimal invasive surgery and Enhanced Recovery After Surgery are useful strategies. The ORANGE II PLUS Trial will provide evidence on patient recovery, oncological outcomes, morbidity, cosmesis and health economics after open and laparoscopic hemihepatectomy in an ERAS setting. 


\section{REFERENCES}

1. Ruers, T. and R.P. Bleichrodt, Treatment of liver metastases, an update on the possibilities and results. Eur J Cancer, 2002. 38(7): p. 1023-33.

2. Cuesta, M.A., et al., Limited laparoscopic liver resection of benign tumors guided by laparoscopic ultrasonography: report of two cases. Surg Laparosc Endosc, 1995. 5(5): p. 396-401.

3. Carswell, K.A., et al., Laparoscopic versus open left lateral segmentectomy. BMC Surg, 2009. 9: p. 14.

4. Vigano, L., et al., Laparoscopic liver resection: a systematic review. J Hepatobiliary Pancreat Surg, 2009. 16(4): p. 410-21.

5. Cherqui, D., Laparoscopic liver resection. Br J Surg, 2003. 90(6): p. 644-6.

6. Dagher, l., et al., Laparoscopic versus open right hepatectomy: a comparative study. Am J Surg, 2009. 198(2): p. 173-7.

7. Dagher, I., et al., Laparoscopic major hepatectomy: an evolution in standard of care. Ann Surg, 2009. 250(5): p. 856-60.

8. Abu Hilal, M., et al., Single-centre comparative study of laparoscopic versus open right hepatectomy. J Gastrointest Surg, 2011. 15(5): p. 818-23.

9. Abu Hilal, M., et al., Laparoscopic versus open left lateral hepatic sectionectomy: A comparative study. Eur J Surg Oncol, 2008. 34(12): p. 1285-8.

10. Abu Hilal, M. and N.W. Pearce, Laparoscopic left lateral liver sectionectomy: a safe, efficient, reproducible technique. Dig Surg, 2008. 25(4): p. 305-8.

11. Dagher, I., et al., Laparoscopic liver resection for hepatocellular carcinoma. Surg Endosc, 2008. 22(2): p. 372-8.

12. Dagher, I., et al., Laparoscopic liver resection: results for 70 patients. Surg Endosc, 2007. 21(4): p. 619-24.

13. Lee, K.F., et al., Laparoscopic versus open hepatectomy for liver tumours: a case control study. Hong Kong Med J, 2007. 13(6): p. 442-8.

14. O'Rourke, N., et al., Laparoscopic resection of hepatic colorectal metastases. HPB (Oxford), 2004. 6(4): p. 230-5.
15. Troisi, R., et al., The value of laparoscopic liver surgery for solid benign hepatic tumors. Surg Endosc, 2008. 22(1): p. 38-44.

16. Nguyen, K.T., T.C. Gamblin, and D.A. Geller, World review of laparoscopic liver resection-2,804 patients. Ann Surg, 2009. 250(5): p. 831-41.

17. Bryant, R., et al., Laparoscopic liver resection-understanding its role in current practice: the Henri Mondor Hospital experience. Ann Surg, 2009. 250(1): p. 103-11.

18. Laurence, J.M., et al., Laparoscopic hepatectomy, a systematic review. ANZ J Surg, 2007. 77(11): p. 948-53.

19. Alkari, B., A. Owera, and B.J. Ammori, Laparoscopic liver resection: preliminary results from a UK centre. Surg Endosc, 2008. 22(10): p. 2201-7.

20. Fearon, K.C., et al., Enhanced recovery after surgery: a consensus review of clinical care for patients undergoing colonic resection. Clin Nutr, 2005. 24(3): p. 466-77.

21. Kehlet, $\mathrm{H}$. and D.W. Wilmore, Multimodal strategies to improve surgical outcome. Am J Surg, 2002. 183(6): p. 630-41.

22. Wilmore, D.W. and H. Kehlet, Management of patients in fast track surgery. Bmj, 2001. 322(7284): p. 473-6.

23. Lassen, K., et al., Consensus review of optimal perioperative care in colorectal surgery: Enhanced Recovery After Surgery (ERAS) Group recommendations. Arch Surg, 2009. 144(10): p. 961-9.

24. Basse, L., J.L. Madsen, and H. Kehlet, Normal gastrointestinal transit after colonic resection using epidural analgesia, enforced oral nutrition and laxative. Br J Surg, 2001. 88(11): p. 1498-500.

25. Basse, L., et al., Accelerated postoperative recovery programme after colonic resection improves physical performance, pulmonary function and body composition. Br J Surg, 2002. 89(4): p. 446-53.

26. Delaney, C.P., et al., Prospective, randomized, controlled trial between a pathway of controlled rehabilitation with early ambulation and diet and traditional postoperative care after laparotomy and intestinal resection. Dis Colon Rectum, 2003. 46(7): p. 851-9.

27. Zutshi, M., et al., Randomized controlled trial compar- 
ing the controlled rehabilitation with early ambulation and diet pathway versus the controlled rehabilitation with early ambulation and diet with preemptive epidural anesthesia/analgesia after laparotomy and intestinal resection. Am J Surg, 2005. 189(3): p. 268-72.

28. van Dam, R.M., et al., Initial experience with a multimodal enhanced recovery programme in patients undergoing liver resection. Br J Surg, 2008. 95(8): p. 969-75. 29. Stoot, J.H., et al., The effect of a multimodal fast-track programme on outcomes in laparoscopic liver surgery: a multicentre pilot study. HPB (Oxford), 2009. 11(2): p. 140-4.

30. Maessen, J., et al., A protocol is not enough to implement an enhanced recovery programme for colorectal resection. Br J Surg, 2007. 94(2): p. 224-31.

31. McCulloch, P., Developing appropriate methodology for the study of surgical techniques. J R Soc Med, 2009. 102(2): p. 51-5.

32. Gore, S.M., The consumer principle of randomisation. Lancet, 1994. 343(8888): p. 58.

33. Brewin, C.R. and C. Bradley, Patient preferences and randomised clinical trials. Bmj, 1989. 299(6694): p. 313-5. 34. Dindo, D., N. Demartines, and P.A. Clavien, Classification of surgical complications: a new proposal with evaluation in a cohort of 6336 patients and results of a survey. Ann Surg, 2004. 240(2): p. 205-13.

35. van den Broek, M.A., et al., Development of a composite endpoint for randomized controlled trials in liver surgery. Br J Surg, 2011. 98(8): p. 1138-45.

36. Lamers, L.M., et al., The Dutch tariff: results and arguments for an effective design for national EQ-5D valuation studies. Health Econ, 2006. 15(10): p. 1121-32.

37. Lamers, L.M., et al., [Measuring the quality of life in economic evaluations: the Dutch EQ-5D tariff]. Ned Tijdschr Geneeskd, 2005. 149(28): p. 1574-8.

38. Langenhoff, B.S., et al., Quality of life after surgical treatment of colorectal liver metastases. Br J Surg, 2006. 93(8): p. 1007-14.

39. Blazeby, J.M., et al., Validation of the European Organization for Research and Treatment of Cancer QLQ-
LMC21 questionnaire for assessment of patient-reported outcomes during treatment of colorectal liver metastases. Br J Surg, 2009. 96(3): p. 291-8.

40. Polle, S.W., et al., Body image, cosmesis, quality of life, and functional outcome of hand-assisted laparoscopic versus open restorative proctocolectomy: long-term results of a randomized trial. Surg Endosc, 2007. 21(8): p. 1301-7.

41. Dunker, M.S., et al., Cosmesis and body image after laparoscopic-assisted and open ileocolic resection for Crohn's disease. Surg Endosc, 1998. 12(11): p. 1334-40.

42. Oostenbrink, J.B., M.A. Koopmanschap, and F.F. Rutten, Standardisation of costs: the Dutch Manual for Costing in economic evaluations. Pharmacoeconomics, 2002. 20(7): p. 443-54.

43. Hoër, J., et al., Einflussfaktoren der narbenhernienenstehung retrospektive untersuchung an 2.983 laparotomierten patienten über einen zeitraum vno 10 jahren. Chirurg 2002. 73: p. 474-480.

44. Andersen, L.P., et al., Incisional hernia after open versus laparoscopic sigmoid resection. Surg Endosc, 2008. 22(9): p. 2026-9.

45. D'Angelica, M., et al., Optimal abdominal incision for partial hepatectomy: increased late complications with Mercedes-type incisions compared to extended right subcostal incisions. World J Surg, 2006. 30(3): p. 410-8.

46. Togo, S., et al., Outcome of and risk factors for incisional hernia after partial hepatectomy. J Gastrointest Surg, 2008. 12(6): p. 1115-20.

47. Paice, J.A. and F.L. Cohen, Validity of a verbally administered numeric rating scale to measure cancer pain intensity. Cancer Nurs, 1997. 20(2): p. 88-93.

48. McGuire, D.B., Comprehensive and multidimensional assessment and measurement of pain. J Pain Symptom Manage, 1992. 7(5): p. 312-9.

49. Dorrepaal, K.L., N.K. Aaronson, and F.S. van Dam, Pain experience and pain management among hospitalized cancer patients. A clinical study. Cancer, 1989. 63(3): p. 593-8.

50. Jensen, M.P., The validity and reliability of pain meas- 
ures in adults with cancer. J Pain, 2003. 4(1): p. 2-21.

51. Krebs, E.E., T.S. Carey, and M. Weinberger, Accuracy of the pain numeric rating scale as a screening test in primary care. J Gen Intern Med, 2007. 22(10): p. 1453-8.

52. Suurmeijer, T.P., et al., The Groningen Activity Restriction Scale for measuring disability: its utility in international comparisons. Am J Public Health, 1994. 84(8): p. 1270-3.

53. Snapinn, S.M., Monitoring clinical trials with a conditional probability stopping rule. Stat Med, 1992. 11(5): p. 659-72.

54. van Breukelen, G.J., M.J. Candel, and M.P. Berger, Relative efficiency of unequal versus equal cluster sizes in cluster randomized and multicentre trials. Stat Med, 2007. 26(13): p. 2589-603.

55. van den Broek, M.A., et al., Feasibility of randomized controlled trials in liver surgery using surgery-related mortality or morbidity as endpoint. Br J Surg, 2009. 96(9): p. 1005-14.

56. Koffron, A.J., et al., Evaluation of 300 minimally invasive liver resections at a single institution: less is more. Ann Surg, 2007. 246(3): p. 385-92; discussion 392-4.

57. Vigano, L., et al., The learning curve in laparoscopic liver resection: improved feasibility and reproducibility. Ann Surg, 2009. 250(5): p. 772-82.

58. Buell, J.F., et al., The international position on laparoscopic liver surgery: The Louisville Statement, 2008. Ann Surg, 2009. 250(5): p. 825-30.

59. McCulloch, P., et al., Randomised trials in surgery: problems and possible solutions. Bmj, 2002. 324(7351): p. $1448-51$.

60. Kazaryan, A.M., et al., Laparoscopic liver resection for malignant and benign lesions: ten-year Norwegian single-center experience. Arch Surg. 145(1): p. 34-40.

61. Topal, B., et al., Laparoscopic versus open liver resection of hepatic neoplasms: comparative analysis of shortterm results. Surg Endosc, 2008. 22(10): p. 2208-13.

62. Pessaux, P., et al., Randomized clinical trial evaluating the need for routine nasogastric decompression after elective hepatic resection. Br J Surg, 2007. 94(3): p. 297-
303.

63. Ito, K., et al., Laparoscopic versus open liver resection: a matched-pair case control study. J Gastrointest Surg, 2009. 13(12): p. 2276-83.

64. Castaing, D., et al., Oncologic results of laparoscopic versus open hepatectomy for colorectal liver metastases in two specialized centers. Ann Surg, 2009. 250(5): p. 84955.

65. Reich, H., et al., Laparoscopic excision of benign liver lesions. Obstet Gynecol, 1991. 78(5 Pt 2): p. 956-8.

66. Gagner, M., M. Rheault, and J. Dubuc, Laparoscopic partial hepatectomy for liver tumor. Surg Endosc, 1992(6): p. 97-98.

67. Hashizume, M., et al., Laparoscopic hepatic resection for hepatocellular carcinoma. Surg Endosc, 1995. 9(12): p. 1289-91.

68. Rau, H.G., et al., Laparoscopic liver resection with the water-jet dissector. Surg Endosc, 1995. 9(9): p. 1009-12.

69. Huscher, C.G., et al., Current position of advanced laparoscopic surgery of the liver. J R Coll Surg Edinb, 1997. 42(4): p. 219-25.

70. Samama, G., et al., Laparoscopic anatomical hepatic resection. Report of four left lobectomies for solid tumors. Surg Endosc, 1998. 12(1): p. 76-8.

71. O'Rourke, N. and G. Fielding, Laparoscopic right hepatectomy: surgical technique. J Gastrointest Surg, 2004. 8(2): p. 213-6.

72. Nguyen, K.T., et al., Comparative benefits of laparoscopic vs open hepatic resection: a critical appraisal. Arch Surg, 2011. 146(3): p. 348-56.

73. Gayet, B., et al., Totally laparoscopic right hepatectomy. Am J Surg, 2007. 194(5): p. 685-9.

74. Topal, B., R. Aerts, and F. Penninckx, Laparoscopic intrahepatic Glissonian approach for right hepatectomy is safe, simple, and reproducible. Surg Endosc, 2007. 21(11): p. 2111.

75. Reddy, S.K., A. Tsung, and D.A. Geller, Laparoscopic Liver Resection. World J Surg, 2010.

76. Nguyen, K.T., et al., Minimally invasive liver resection for metastatic colorectal cancer: a multi-institutional, 
international report of safety, feasibility, and early outcomes. Ann Surg, 2009. 250(5): p. 842-8.

77. Satava, R.M., Identification and reduction of surgical error using simulation. Minim Invasive Ther Allied Technol, 2005. 14(4): p. 257-61.

78. Kazaryan, A.M., et al., Comparative evaluation of laparoscopic liver resection for posterosuperior and anterolateral segments. Surg Endosc, 2011. 25(12): p. 3881-9. 79. Strasberg, S.M., D.C. Linehan, and W.G. Hawkins, The accordion severity grading system of surgical complications. Ann Surg, 2009. 250(2): p. 177-86.

80. Porembka, M.R., et al., Quantitative weighting of postoperative complications based on the accordion severity grading system: demonstration of potential impact using the american college of surgeons national surgical quality improvement program. J Am Coll Surg, 2010. 210(3): p. 286-98.

81. Halm, J.A., et al., Incisional hernia after upper abdominal surgery: a randomised controlled trial of midline versus transverse incision. Hernia, 2009. 13(3): p. 275-80. 82. Bedossa, P. and T. Poynard, An algorithm for the grading of activity in chronic hepatitis C. The METAVIR Cooperative Study Group. Hepatology, 1996. 24(2): p. 289-93. 83. Jarnagin, W.R., et al., Improvement in perioperative outcome after hepatic resection: analysis of 1,803 consecutive cases over the past decade. Ann Surg, 2002. 236(4): p. 397-406; discussion 406-7.

84. Stoot, J.H., et al., The introduction of a laparoscopic liver surgery programme: a cost analysis of initial experience in a university hospital. Scand J Surg, 2012. 101(1): p. 32-7.

85. Vlug, M.S., et al., Laparoscopy in Combination with Fast Track Multimodal Management is the Best Perioperative Strategy in Patients Undergoing Colonic Surgery: A Randomized Clinical Trial (LAFA-study). Ann Surg, 2011. 86. Lin, N.C., H. Nitta, and G. Wakabayashi, Laparoscopic major hepatectomy: a systematic literature review and comparison of 3 techniques. Ann Surg, 2013. 257(2): p. 205-13.

87. van Dam, R.M., et al., Open versus laparoscopic left lateral hepatic sectionectomy within an enhanced recovery ERAS(R) programme (ORANGE II-trial): study protocol for a randomised controlled trial. Trials, 2012. 13: p. 54. 88. Wong-Lun-Hing, E.M., et al., A survey in the hepatopancreatobiliary community on ways to enhance patient recovery. HPB (Oxford), 2012. 14(12): p. 818-27.

89. Tzanis, D., N.Shivarthirthan, A. Laurent, Abu Hilal, O. Soubrane, A.M. Kazaryan, et all., European Experience of laparoscopic major hepatectomy. J. Hepatobiliary Pancreat Sci, 2012.

90. Hendry, P.O., et al., Randomized clinical trial of laxatives and oral nutritional supplements within an enhanced recovery after surgery protocol following liver resection. Br J Surg, 2010. 97(8): p. 1198-206.

91. Fong, Y., et al., Long-term survival is superior after resection for cancer in high-volume centers. Ann Surg, 2005. 242(4): p. 540-4; discussion 544-7. 


\section{GENERAL DISCUSSION AND PERSPECTIVES: TOWARDS EVIDENCE BASED LIVER SURGERY}

CHAPTER XII

General discussion adapted from

SARCOPENIA NEGATIVELY AFFECTS PREOPERATIVE TOTAL FUNCTIONAL LIVER VOLUME IN PATIENTS UNDERGOING LIVER RESECTION

S.A. Dello, T.M. Lodewick, R.M. van Dam, K.W. Reisinger, M.A. van den Broek,

M.F. von Meyenfeldt, M.H.A. Bemelmans, S.W.M. Olde Damink, C.H.C. Dejong

HPB (Oxford). 2013 Mar;15(3):165-9

DOI: dx.doi.org/10.1111/j.1477-2574.2012.00517.x

THE INTRODUCTION OF A LAPAROSCOPIC LIVER SURGERY PROGRAMME: A COST ANALYSIS OF INITIAL EXPERIENCE IN A UNIVERSITY HOSPITAL

J.H. Stoot, R.M. van Dam, R.J. Coelen, B. Winkens, S.W.M. Olde Damink, M.H.A. Bemelmans, C.H.C. Dejong.

Scand J Surg. 2012;101(1):32-7

DOI: Pubmed ID 22414466

FEASIBILITY OF RANDOMIZED CONTROLLED TRIALS IN LIVER SURGERY USING SURGERY-RELATED MORTALITY OR MORBIDITY AS ENDPOINT M.A. van den Broek, R.M. van Dam, M. Malagó, C.H.C. Dejong, G.J. van Breukelen,

S.W.M. Olde Damink

BJS 2009 96(9):1005-14

DOI: dx.doi.org/10.1002/bjs.6663

\section{DEVELOPMENT OF A COMPOSITE ENDPOINT FOR RANDOMIZED CONTROLLED TRIALS IN LIVER SURGERY}

M.A. van den Broek, R.M. van Dam, G.J. van Breukelen, M.H.A. Bemelmans, E. Oussoultzoglou, P. Pessaux, C.H.C. Dejong, N. Freemantle, S.W.M. Olde Damink

BJS 2011; 98(8):1138-45

DOI: dx.doi.org/10.1002/bjs.7503

IDENTIFICATION AND VALIDATION OF RISK FACTORS FOR POSTOPERATIVE INFECTIOUS COMPLICATIONS FOLLOWING HEPATECTOMY

P. Pessaux, M.A. van den Broek, T. Wu, S.W.M. Olde Damink, T. Piardi, C.H.C. Dejong, D. Ntourakis, R.M. van Dam

J Gastrointestinal Surgery ePub May 2013

DOI: dx.doi.org/10.1007/s11605-013-2226-1 


\section{BACKGROUND}

Less than 200 years after the first public demonstration of the use of anaesthesia in a human undergoing an operation, an average of 235 million surgical procedures are performed worldwide annually[1, 2]. This number serves to underpin the enormous historical development in the surgical field. The first liver resection was performed less than 30 years after the inception of anesthesia, and this must be regarded as an enormously brave step at that time when much less sophisticated tools were available for such major surgery. Over the past century, the number of liver resections performed globally has increased immensely. It is only by the continuous striving for improvement in patient care that this has all become possible.

Research, a term generally used for basic and translational research as well as for process development of clinical care, plays a crucial role in advancing the art and science of liver surgery. In the past, the focus in liver surgical research has been merely content driven, i.e. focused at new discoveries. In more recent years, attention has increasingly shifted towards how the results of this research can be made available more quickly to the patient. This societal and governmentally driven attention on translating knowledge from bench to beside has intensified attention on the conduct of clinical trials, and the development and implementation of guidelines. Generally speaking, this strategy leads to a knowledge base of what is best for most with acceptable costs for society. This does, however, not take into account the individualization of patient care. Increasing knowledge on genetic predisposition, specific patient conditions such as co-morbidity, exercise tolerance or nutritional status, responsiveness to pre-operative therapies, individual circumstances particular to the patient or the doctor or institution (Figure 1), call for a much more personalized approach of the patient with a liver tumor.

Many patients with cancer undergo therapeutic interventions (e.g. surgery, radio- or chemotherapy), and the way they are prepared for this as well as the organization of care after such interventions is key in determining outcome. The 'average patient' does not exist, and therefore it is crucially important to individualize patient care against the background of best evidence. In this context, it is important to realize that lack of evidence of effect in specific circumstances should not be confused with evidence of lack of effect. As patients are extremely vulnerable at the extremes of age and physionomy, the young are much more resilient to high impact treatments. The elderly and the undernourished patients, but equally the obese patients are at particular risk of adverse outcome after interventions. The same holds true for those who are immune-suppressed due to chemotherapy. Prolonged and intensive treatments strongly influence the nutritional status and metabolism of these patients and attention towards supporting metabolism and maintaining health and fitness should be at the heart of individualized patient care. It is for this reason that the Health 
Inspectorate in the Netherlands has imposed the assessment of nutritional status prior to surgical interventions.

\begin{tabular}{|c|c|c|c|c|}
\hline Basic science & Translation & Clinical trials & $\begin{array}{l}\text { Guidelines \& } \\
\text { implementation }\end{array}$ & $\begin{array}{l}\text { Individualized } \\
\text { Care }\end{array}$ \\
\hline
\end{tabular}

Figure 1. Individualization of patient care

Until recently, it was common practice to keep patients fasted before and after surgical interventions for prolonged periods, and this inevitably affected the patient's nutritional status. An important effect of prolonged fasting is the induction of insulin resistance postoperatively.

In the Maastricht University Medical Center, there is considerable experience with implementation of best practice peri-operative care for patients: the so-called Enhanced Recovery After Surgery (or Fast Track) programs (www.erassociety.org) [3-6] This peri-operative care program leads to faster recovery due to optimal pain management, early feeding, less tubes and drains, avoidance of insulin resistance and active mobilization after surgery. Besides a faster recovery the implementation of these programs also led to a reduction of perioperative adverse events[7]. Since as a consequence, patients leave the hospital quicker and in a better physical condition this may allow for an earlier start of adjuvant chemotherapy in patients that underwent cancer surgery. It is already known for a decade that postoperative complications after hepatic resection lead to a reduced disease free survival and overall survival[8]. Recently this effect on survival was shown again, even more specifically for surgical site infections $[9,10]$. So far it is unclear if the reduction in complications leads to less suppression of the cell mediated immunity or that patients without complications have a higher and quicker access rate to neo-adjuvant chemotherapy, both potentially affecting the long-term oncological results.

It has been suggested that Fast Track care also improves long-term oncological outcome, probably as consequence of the aforementioned principles or even by the effect of the epidural anaesthesia often used in enhanced recovery programmes[11]. For patients that are malnourished, preoperative nutritional support has been shown to improve outcome of surgery. Likewise, targeted interventions in the obese and immune-suppressed may help improve outcome. As body composition can relatively easily be assessed in patients by muscle mass measurement using CT [12], such targeted interventions are feasible. Similar interventions may be appropriate for the larger groups of cancer patients undergoing radiotherapy and chemotherapy.

In this general discussion, several of the above issues will be discussed, and directions to- 
wards future research and individualization of patient care will be proposed. Focus is on patients who otherwise have a normal liver, i.e. the discussion will not address the patient with a cirrhotic liver.

\section{ADVANCING THE LIMITS OF RESECTIONAL LIVER SURGERY}

\section{ADVANCES IN SURGICAL TECHNIQUE}

In 1886, Carl Langenbuch performed the first liver resection at the Lazarus-Krankenhaus in Berlin: a relatively simple resection of a part of the left lobe of the liver. Since then, we have seen an immense and accelerating evolution of surgical techniques. This has been achieved by the initiatives, perseverance and courage of the early pioneers in the field, but equally due to the fact that more sophisticated surgical devices and insight in liver anatomy became available. In addition to this, also anesthesiology has evolved immensely and this has facilitated the control of bleeding during the surgical procedure, which at the beginning was the major cause of fatal outcome. Further, the introduction of progressively more effective chemotherapies, such as oxaliplatin and irinotecan, and biological response modifying agents, such as bevacizumab, cetuximab and panitumumab, has increased our ability to treat hepatic malignancies [13]. Finally, local ablative techniques have evolved and a whole range of radiological interventional procedures have been introduced [14].

Over the years, more major resections were performed and shown feasible and safe [15, 16]. Staged hepatectomies, repeat liver resections, two stage liver resections after unilateral portal vein ligation, liver resections after preoperative portal vein embolization and the liver first approach have all seen the light. Combinations with needle radiofrequency ablation, transarterial embolization, in situ liver perfusion and gamma knife or stereotactic radiotherapy have been introduced. For patients who are jaundiced, endoscopic or percutaneous biliary drainage may optimize them for surgery. All of these have remarkably increased the surgical armamentarium to combat primary or secondary liver tumors. Among the more recently popularized strategies are the liver resections combined with other resections, as well as combined with vascular (arterial or portal vein) reconstructions. Thus, e.g. in the treatment of hilar cholangiocarcinoma, the teams from from Nagoya (Japan) and Berlin (Germany) have proposed the extended hepatectomy plus bile duct resection and portal vein resection and reconstruction to achieve more radical resections [17-19]. This does lead to more $\mathrm{R} 0$ resections, but it also carries significant morbidity and mortality and should only be performed in experienced centers.

One of the more recent potential advances is the very recently introduced ALPPS procedure [20]. The latter entails splitting the liver in situ, ligating the portal vein to the side to 
be resected and then closing the abdomen, to re-operate one week later and take out the (usually right) hemi-liver to be resected. This procedure has been shown to induce immense and rapid hypertrophy of the future remnant liver, even after previous portal vein embolisation with failing hypertrophy [21, 22]. As the first reports of the use of the ALPPS procedure show significant morbidity and mortality, its indication and use is currently being debated in the HPB community (E-AHPBA 2013, Belgrade, Serbia, ALPPS session). The indication for an ALPPS procedure might be restricted to large tumours at the border of the falciform ligament or to those patients that fail to respond to portal venous occlusion procedures. In any case, the rapid introduction of the ALPPS procedure shows that close monitoring of the effect of any new technique in surgery is warranted. Large prospective data registries are probably best suitable for this [23].

\section{ASSESSING OUTCOMES OF NEW SURGICAL APPROACHES}

The problem with most of the new surgical procedures is that they are introduced into the field of liver surgery in a rather uncontrolled way [23]. None of the procedures stipulated above have been subjected to proper testing in randomized controlled trials, which in the current era would seem to be the most appropriate way of testing new surgical procedures. On the one hand, the creativeness of individuals from whose brain the inception of some of the above procedures comes is one of the virtues of the art and science of surgery. On the other hand, the lack of proper testing, and uncontrolled introduction of new surgical techniques into the field of liver surgery potentially exposes patients to unnecessary risks and requires change. The introduction of standards in reporting RCTs such as the Consolidated Standards of Reporting Trials (CONSORT) has led to the necessity of accurately defining primary and, if required, secondary outcomes [24].

A major problem is that trials are often difficult to perform in surgery. First of all, the incidence of some of the most important outcomes in liver surgery, such as bile leak, liver failure and mortality is too low to make a trial realistic in terms of sufficient patient enrollment or trial duration. Such a trial would frequently even be virtually impossible in a large collaborative multinational network [25]. Also, contemporary Good Clinical Practice regulations for clinical trials and data management and absence of international funding bodies make multinational trials difficult to start. Another point is the lack of clear definitions of such endpoints. The International Study Group of Liver Surgery (ISGLS) has focused in the past years on developing common definitions on endpoints in liver surgery [26-28]. Such definitions of single, individual endpoints improve the possibilities to compare results between trials and use these later in meta-analyses. Likewise, the anatomical classification of liver resections has been agreed upon in the IHPBA consensus statement [29]. This more accurately defines the exact procedures that were performed within a given trial and therefore is another example of how comparison of outcomes of trials can be improved. 
In recent years, we have focused on developing a composite endpoint (CEP) that enables to reduce the sample size required for trials in HPB surgery [30]. A composite, liver specific endpoint was developed, which consists of a combination of liver specific individual single endpoints, with each a rather low incidence following liver resections. If combined, the incidence of the liver specific (combined) endpoint increases and this facilitates the feasibility of conducting a randomized trial with a considerable smaller sample size. Moreover, consistent use of this liver surgery specific CEP may improve the quality of future meta-analyses, since it ensures that each individual endpoint can be studied and it reduces the missing data problems that currently undermine the validity of meta-analyses of heterogeneous studies. Hence, CEPs may increase the feasibility of conducting randomized clinical trials and meta-analyses in surgery. Recently, a CEP for resection surface related complications after liver resection, including biliary leakage, bleeding or abscess formation at the resection surface, was used in the Dutch multicenter randomized controlled FRESCO trial. This trial showed no added value of the use of liquid fibrin sealant in preventing bile leaks, bleeds or abscesses after liver resection [31].

As an alternative approach, we have explored the feasibility of using surrogate endpoints. These may be used either as primary, or a secondary outcome [24]. As the incidence of the surrogate endpoint often is much higher than those of the traditional clinical endpoints such as morbidity and mortality, the sample size for trials based on surrogate endpoints is usually considerably smaller. However, in a systematic review of 49 trials in literature, we found that numerous surrogate endpoints were used (particularly biochemical markers), but non were validated [32].

A dominant, and unsolved problem in surgical trials, particularly if they address a technical issue, is the 'Blinding' of patients, caregivers (doctors, nurses etc), and outcome assessors, as well as the right choice of the 'Placebo'. In the current era of explicit interest and evolution in laparoscopic liver surgery, where often an open procedure is compared with a minimally invasive approach, it is important to realize that it is difficult to blind patients, caregivers and outcome assessors for the type of intervention that the patient underwent. Big wound dressings are only partially helpful in solving this problem. Equally, it may be considered unethical by Institutional Review boards to use sham drains (inserted only subcutaneously), to just name a few of the problems with surgical trials. In this context, it is important to stress that there is hardly any agreement on how a particular procedure should be performed laparoscopically (there is more agreement for open procedures). Thus, for the laparoscopic left lateral sectionectomy, that is now claimed by some to be first choice approach for tumors in segment 2-3 of the liver [33], the only parts of the procedure that are currently fairly standardized are the final two steps: identifying the left hepatic vein and transecting this by stapling. The other steps of this surgical procedure are performed in a variety of manners across the globe (the open approach is more standardized). This serves just as an example 
of the lack of standardization in minimally invasive liver surgery, which is an impediment for trial conduct.

In many trials on perioperative care, length of hospital stay is used as the primary outcome. This may not be justified as length of stay is not an accurate outcome measure [34]. Length of stay in hospital is influenced by many variables such as patient or family expectations, preoperative counseling, doctor's and nurses' attitudes, and there may be strong geographical influences across the world. This too, makes comparison between trial results from different countries difficult if not impossible.

As an alternative, the ERAS group has popularized the concept of pre-defined discharge criteria, that assess if a patient is pain free with oral analgesics only, without intravenous infusions, eating and drinking and independently mobile at the preoperative level. Others have worked on fine-tuning this concept by re-naming this 'time to readiness for discharge (TTRD)'. Interestingly, recent data showed that blinded assessment of TTRD by a dedicated team results in considerably less variation in this outcome if compared with LOS. This in turn may further help reduce sample size for trials $[35,36]$.

\section{REDUCING THE RISKS OF LIVER SURGERY}

\section{ASSESSMENT OFTHE PATIENT}

In the past, liver surgery was not offered to the elderly patients and not to those with cardiopulmonary comorbidity[37]. This obviously was not based on objective criteria and it is highly likely that many patients under these rules have been denied operations that could have cured them. It has been shown by now that liver surgery in patients with otherwise normal liver function is perfectly safe and feasible even in octogenarians. Assessment of fitness to undergo a surgical procedure, or maybe even more importantly, to withstand the stress of the potential complications of this procedure, should be based on objective criteria. Evaluation of the patient by preoperative cardiopulmonary exercise testing has recently been advocated [37]. This implies measuring where the anaerobic threshold of a particular patient is during exercise and objectively assesses the capacity of a patient to withstand the insult of major surgery and its complications.

At the extremes of human physionomy, it seems that both the obese and the cachectic patient are at particular risk during liver surgery. First, obesity, steatosis and the associated metabolic syndrome, as well as steatohepatitis affect hepatic function adversely and have been suggested to impact negatively on outcome after liver surgery [38-40]. An increased risk of developing primary malignant liver tumours has been found in patients with hepatic steatosis as well as in those with metabolic syndrome [41]. This effect of the metabolic syn- 
drome may already be present before the effects on the liver are visible on imaging modalities. It has been shown in recent animal experiments [42], that nutritional interventions may help reverse hepatic steatosis and that this positively affects hepatic functional recovery after liver resection. It is evident that in the global obesity pandemia, the assessment and treatment of hepatic steatosis becomes more and more important. It may well be that future improvements in CT and/or MRI scanning will help to accurately assess the steatosis or steatohepatitis in the patient's liver prior to liver surgery by using e.g. liver spleen enhancement ratios [43].

Second, patients who have lost too much weight are at increased risk if they undergo (major) liver surgery. The anamnestic assessment of weight loss may shed some light on the patient's general condition and ability to cope with the injury of liver surgery. However, this is not a very scientific, reproducible, or reliable assessment. Recently, it has been suggested that the CT-based volumetric measurement of skeletal muscle mass at the third lumbar vertebral level provides a reliable and reproducible measure of whole body muscle mass [12]. It has been shown that patients with severe reductions in muscle mass, termed sarcopenic patients, are at increased risk if they undergo major liver surgery [44]. Recent insights suggest that patients with sarcopenia on CT scan may have a reduced regeneration of liver tissue after liver resections [45]. There is now a growing body of evidence that patients who are obese actually may have a strongly reduced muscle mass (sarcobesity) [46]. Even more so, weight losing obese cancer patients, the so-called sarcopenic obese cachectic patients, have poor post-operative outcome $[47,48]$. This has been explained by the mild inflammatory state that is induced by the obesity, which primes the patient for the systemic inflammation that is related to cancer cachexia. It becomes evident that we are only at the verge of understanding the individual risks and consequently the needs of patients scheduled for major liver surgery.

\section{VOLUMETRIC ASSESSMENT OFTHE LIVER PRIORTO SURGERY}

In recent years, enormous advances have been made in CT scan technology and the current multichannel, multidetector, spiral CT scans allow for the swift acquisition of enormous amounts of data of the total body in a few seconds. This also enables hepatic volumetric assessments in a much more easy, and rapid fashion, but human capital still is a problem, as the radiologist has to spend time on the measurement. One may reduce radiation to patients by using the MRI for the same purpose. However, the problem with the time investment remains unchanged. Currently, several groups are exploring the fully automated pixel-based volumetric assessment of liver and tumor volumes. In this approach, a sensor picks up differences in Hounsfield Units between various areas in the liver and then calculates in an automated way the various areas on CT-images. In patients with normal undamaged liver parenchyma, the current assumption is that following liver resection, at least $30 \%$ residual liver volume must remain to survive and not develop liver failure [49]. In patients 
with impaired liver function, as is seen in patients with cirrhosis of the liver or following prior chemotherapy with consequently steatosis, steatohepatitis or microvascular changes, at least $40 \%$ liver volume should remain to avoid the risk of fatal liver failure.

\section{FUNCTIONAL ASSESSMENT OF THE LIVER PRIORTO SURGERY}

Over the years, the concept of just measuring liver volumes to predict outcome after liver resections has evolved because it became apparent that liver function might vary considerably and hence volume may not equal function. This has driven the continued research to develop a way to assess liver function for the liver as a whole, but equally per volume unit of liver. Classically, many liver surgeons have used the 15-minute indocyanine green retention test (ICG R15) prior to surgery. This test measures the clearance capacity of the liver for indocyanine green and is probably the most commonly used test in patients undergoing liver resection. To determine the maximum possible extent for safe liver resection, Makuuchi et al. and Clavien et al. proposed the use of decision trees that incorporate the ICG R15 [50-52]. The ICG R15 test is relatively simple to perform, but the lack of widespread diffusion into the general HPB community probably testifies of the pitfalls this test may have in terms of reproducibility and accuracy. More recently, the 13C-methacetin LIMAX breath test has been propagated in particular by the Berlin group [53, 54]. Although this test is promising, its wider diffusion has been hampered by the mere fact that the machine required for the test is expensive and the topic maybe subject to vested bias (i.e. those who write about it have a financial interest in it). Moreover the metabolic labeled substrates used for these hepatocellular function assessing breath tests, like 13C-methacetin or 13C-Aminopyrine, are formally not for human use. The surgical team from Amsterdam Medical Center [55] has put a major effort in exploring the use of 19Tc Mebrofenin hepatobiliary scintigraphy for assessing hepatic functional capacity. This test has yielded interesting results in both experimental animal studies and human research [42]. Unfortunately, there is hitherto no ideal test to assess liver function and hence predict outcome following liver resection.

In the past few years, we and others have explored the potential to use hepatic hyaluronic acid clearance as a measure of liver function. Hyaluronic acid clearance by the sinusoidal endothelial cells may be reduced following prior chemotherapy and therefore we have investigated if plasma hyaluronic acid is an effective measure of potential liver damage following chemotherapy and subsequent sinusoidal obstruction syndrome (SOS) [56]. Unfortunately, we could not confirm that hyaluronan might be a very strong predictor of liver function postoperatively. We found that hyaluronic acid was increased in patients with moderate and severe SOS compared with those who had only mild SOS. In the study, hyaluronic acid clearance across the liver showed a tendency to be decreased in those individuals, who turned out to have more severe SOS; however, this failed to attain statistical significance. This may have been due to the small patient groups, in which case a repeat study in a bigger patient cohort may be worthwhile. Some alternative explanations for these findings have been pro- 
posed [13] including portacaval shunting and a role for the primary tumor. For hyaluronic acid, it appears that individual patients may be genetically more susceptible to chemotherapy and resulting SOS. Hence, hyaluronic acid may not be a good marker in some patients but it may be in others [57]. Likewise, we have explored ophthalmate as a potential marker for glutathione depletion and liver function in patients undergoing liver surgery [58]. Results of these studies are awaited.

\section{IMPROVING THE OUTCOME OF LIVER SURGERY: FUTURE PERSPECTIVES}

\section{POPULATION-BASED APPROACH: CENTRALIZATION OF LIVER SURGERY}

It is well known that the results of major surgery in terms of in-hospital mortality are far better in hospitals that perform high volumes of such procedures, most likely because complication management is better in high-volume hospitals [59]. This volume-effect has now been shown in many countries [60] and for many major surgical procedures including pancreatic resections and liver resections, and points to the importance of having a specialized multidisciplinary team looking after patients on a 24/7 basis. In the South of the Netherlands this has resulted in a regionalization of the care for low-volume cancers like esophagus, pancreas and liver cancer in 2009. The Surgical Oncologic Network Limburg was founded and this led to a steep increase in the number of liver resections performed by our unit (Figure 2). In addition to the care organized in a specialized multidisciplinary team, it has been shown that there is also a similar surgeon volume effect [61]. This implies that even in high volume centers, a surgeon who performs a specific high-risk procedure more frequently is likely to have better results. It may well be that for certain tumour types, such as the hilar cholangiocarcinoma (Klatskin tumor), which require complex workup and surgical treatment, a decision should be made to treat them in an even more limited number of centers in a particular country.

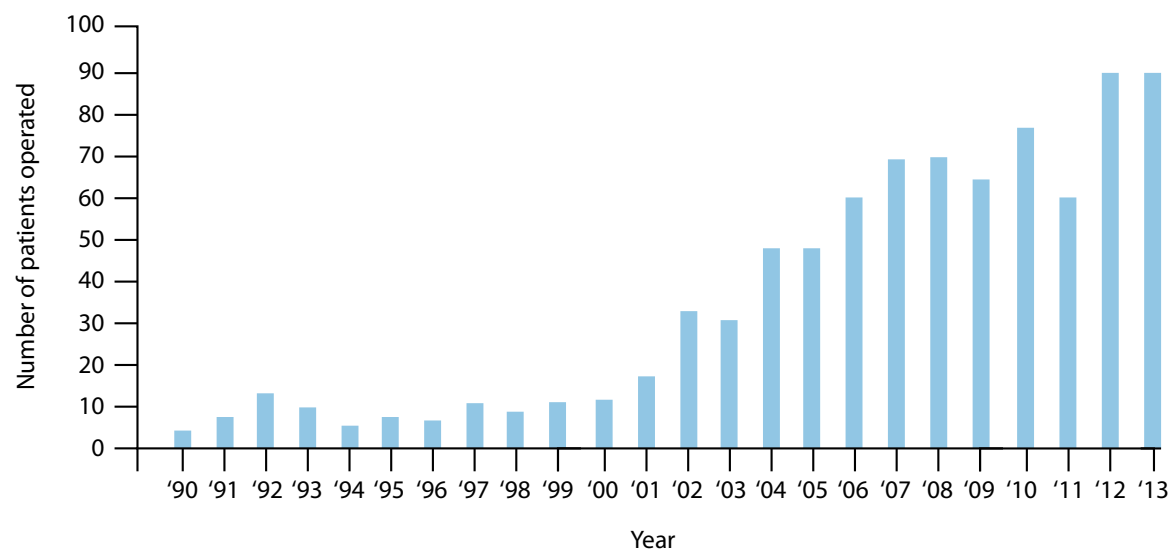

Figure 2. Number of patients undergoing liver resection 


\section{SURGEON-BASED APPROACH:TEACHING AND TRAINING IN A SPECIALIZING ENVIRONMENT}

It is self-evident that the increasing complexity of liver surgery puts a major demand on the technical proficiency of surgeons. This holds true for both open and laparoscopic procedures. Equally, it puts special demands on the teaching and training of young surgeons, particularly because European working time directives have restricted the number of hours a surgical trainee can spend in hospital. In addition to the tendency to perform more and more complex open surgery, there has been a rapid shift to less invasive surgery also in the field of liver surgery. This has created a particular problem, as most current experienced liver surgeons were in their training as a liver surgeon never really exposed to doing minimally invasive liver surgery in a structured manner. Thus, in parallel, experienced liver surgeons with little experience in laparoscopic surgery are in charge of training young surgeons with very little experience in liver surgery, but who have been trained in the era of laparoscopic surgery. To bridge this gap, a constructive and continuous effort has to be made to train experienced and young surgeons in parallel. In the past 2 decades, examples have been created e.g. in France (IRCAD, Strasbourg). Here, a laparoscopic training center has been initiated which enables the constructive, and structured training of surgeons with varying levels of experience in laparoscopic surgery and liver surgery. This undertaking has been very successful and has led to similar initiatives to set up training centers in Korea and Brazil. Continued proctoring should lead to the further safe implementation of the laparoscopic approach in liver surgery. It is highly likely that this will in due time also apply to robotic surgery.

\section{PATIENT-BASED APPROACH: INDIVIDUALIZED PATIENT CARE}

For decades, the medical community has merely looked at patient groups, and treatments have been proposed for such groups of patients. Thus, it is clear that the addition of the newer chemotherapeutics oxaliplatin and irinotecan to the portfolio of treatments including the classical chemotherapy based on fluorouracil has dramatically improved survival of the group of patients with liver metastases. Likewise, the recent introduction of bevacizum$\mathrm{ab}$ and cetuximab, and probably also panitumumab, has led to an improvement in survival perspective in this group of patients. It is only recently that our knowledge has increased on individual responsiveness to certain types of treatment. Thus, as an example, patients who are kRAS mutant may not be responsive to cetuximab. In this context, it may be superfluous to subject patients to a treatment including cetuximab. Hence the chemotherapy approach needs to be personalized, and results of trials (no effect/ positive effect) are not just applicable to every patient. In the near future PETCT or PETMRI may be useful to select responders after just one or two cycles of chemotherapy. In the longer term genetic profiling may be of use.

In recent years, patient safety has been justly put increasingly on the agenda. The use of structured informed consent, check lists and sign in and sign out procedures as well as stopping moments in surgical procedures has contributed strongly to patient safety[62]. This will no doubt be reflected in patient reported outcome assessment 

ICAL RESEARCH

It is known that dogma still has a very strong influence on surgical care. The strong beliefs, do's and don'ts learned during the master apprentice relationship in surgical training, are keeping surgeons from implementing new knowledge. In 2005 an editorial in the British Medical Journal stated that "the immediate challenge to improving the quality of surgical care is not discovering new knowledge, but rather how to integrate what we already know into practice“[63]. Recently we have shown that even today it still takes more than a decade to implement knowledge of published clinical trials into HPB surgical practice[64]. Collaborative initiatives, as the Dutch Liver Working Group, defining datasets for clinical multicenter trials and continuous national audits of the Dutch Institute of Clinical Auditing or the international Enhanced Recovery after Surgery group, may help to accelerate the implementation of trial outcomes.

A prerequisite for all trials and audits to be unconditionally comparable is the use of globally accepted well-defined outcomes and classification systems. It contributes to improved health care quality control and facilitates surgical research and clinical development. The International Study Group of Liver Surgery (ISGLS) has designed consensus-based definitions of a few post-operative complications in liver surgery that can be used. The Accordion, modified Clavien-Dindo-Strasberg, classification system of postoperative surgical complications is the best-defined classification system but disregards medical and intraoperative adverse events[65]. Sofar the Satava-classification system for intraoperative adverse events, may be the most suitable to fill that deficit[66]. Further optimization in worldwide consensus of these systems for registration and classification of perioperative adverse events is required.

\section{IMPROVING PERIOPERATIVE CARE.}

In the field of research concerning improving the perioperative care of the patient, focus has long been on length of stay in hospital as the main outcome. This has been strongly driven by hospital managers, economists and the government, where costs of health care figure prominently on the agenda. As both the laparoscopic approach and the ERAS approach have been associated with reduced length of stay in the hospital, two streams of protagonists have dominated this field: those who propagated ERAS, and those who propagated the laparoscopic approach.

It is only recently that the surgical community has started to grasp the fact that these two approaches are not competitive, but should be considered complementary. In the field of liver surgery, we have piloted in the Netherlands a study where the standardized segment 2-3 liver resection was performed laparoscopically either with or without an ERAS approach [67]. This did not lead to a significant reduction in length of stay in the ERAS group (5 versus 7 days), but functional recovery was significantly faster in the ERAS group. This study had 
some criticism as it was non-randomized and had a number of potential biases (multicenter, national, some centers did not perform laparoscopic liver resections, some no ERAS). It was therefore appropriate to conduct an RCT on laparoscopic left lateral sectionectomy (segment 2-3 resection) either open or laparoscopic within an ERAS approach. This multinational multicenter trial, the ORANGE II trial, is currently ongoing [68].

Unfortunately, trial accrual in this RCT is rather slow and this may be due to the fact that the standard approach to do a left lateral sectionectomy is nowadays felt to be the laparoscopic technique [33]. Moreover, patients are choosing the laparoscopic technique after reading the patient information provided with the trial informed consent procedure.

In investigating internationally what the reasons for the slow accrual were, it transpired from the HPB community that the true laparoscopic protagonists felt that the real issue to conduct a trial on in laparoscopic liver resection would be the right or left hepatectomy laparoscopically or open in an ERAS approach. For this reason we initiated the ORANGE II Plus trial, an additional randomized controlled trial in European expert centers to compare the open and laparoscopic approach in ERAS for right and left hepatectomies.

\section{WHERE DO WE GO?}

With the increasing safety of liver surgery, it is clear that access to this treatment should be optimal. This is currently not the case. In the Netherlands, not all patients who develop recectable liver metastases of colorectal cancer are treated by liver resection. Similar data are available from other countries, making clear that there is considerable under-referral of patients with colorectal liver metastases from district general referring hospitals to tertiary referral liver surgery units [69]. It is also clear that judgment of resectability varies considerably between liver surgeons [70]. Thus, there is considerable room for improvement, and using large national registries such as the Dutch DICA database may provide objective data on this.

With the continuing developments in additive treatments, cancer is more and more becoming a chronic disease. Recent data from large liver resection registries suggest that progression of metastatic disease under chemotherapy should no longer be viewed as a contraindication for liver surgery [71]. Likewise, it has been shown that even R1 resections of liver metastases have an improved long-term oncological outcome[72]. It may well be that liver surgery is cheaper than repeated and prolonged chemotherapy, but data on this are currently lacking.

Clearly, each patient with colorectal cancer metastases, but probably also every patient with a liver tumor, should be discussed at a specialized liver and colorectal cancer multidisciplinary team meeting. Only in this way will patients have access to optimal expertise 
and treatment. This is now also transpiring in national and international guidelines. Current guidelines are too general and this needs to be improved, taking into account ways to adapt treatment proposals to the personal situation of the patient. Ultimately, the treatment proposed to the patients should be tailored and individualized, whereby the acceptance of that proposal by the patients is made by a shared decision of both patient and caregiver. It has become clear that patients often have strong preferences, and their preferences do not always coincide with the prefences of their doctors, the guidelines or the society. In this context it is important to realize that medical specialists, society and patients judge the benefit of a treatment differently. In exploring the limits of resectional liver surgery many members of a multidisciplinary team emphasize what can go wrong while most patients are prepared to take a calculated risk based on what can go well[73]. 


\section{REFERENCES}

1. LeVasseur, R. and S.P. Desai, Ebenezer Hopkins Frost (1824-1866): William T.G. Morton's first identified patient and why he was invited to the Ether demonstration of October 16, 1846. Anesthesiology, 2012. 117(2): p. 238-42. 2. Weiser, T.G., et al., An estimation of the global volume of surgery: a modelling strategy based on available data. Lancet, 2008. 372(9633): p. 139-44.

3. Lassen, K., et al., Consensus review of optimal perioperative care in colorectal surgery: Enhanced Recovery After Surgery (ERAS) Group recommendations. Arch Surg, 2009. 144(10): p. 961-9.

4. Lassen, K., et al., Guidelines for perioperative care for pancreaticoduodenectomy: Enhanced Recovery After Surgery $(\operatorname{ERAS}(\mathrm{R}))$ Society recommendations. World J Surg, 2013. 37(2): p. 240-58.

5. Coolsen, M.M., et al., Systematic Review and Meta-analysis of Enhanced Recovery After Pancreatic Surgery with Particular Emphasis on Pancreaticoduodenectomies. World J Surg, 2013.

6. Coolsen, M.M., et al., A systematic review of outcomes in patients undergoing liver surgery in an enhanced recovery after surgery pathways. HPB (Oxford), 2013. 15(4): p. 245-51.

7. Varadhan, K.K., et al., The enhanced recovery after surgery (ERAS) pathway for patients undergoing major elective open colorectal surgery: a meta-analysis of randomized controlled trials. Clin Nutr, 2010. 29(4): p. 434-40.

8. Laurent, C., et al., Influence of postoperative morbidity on long-term survival following liver resection for colorectal metastases. Br J Surg, 2003. 90(9): p. 1131-6.

9. Mavros, M.N., et al., Impact of complications on longterm survival after resection of colorectal liver metastases. Br J Surg, 2013. 100(5): p. 711-8.

10. Haruki, K., et al., Negative impact of surgical site infection on long-term outcomes after hepatic resection for colorectal liver metastases. Anticancer Res, 2013. 33(4): p. 1697-703.

11. Chen, W.K. and C.H. Miao, The effect of anesthetic technique on survival in human cancers: a meta-analysis of retrospective and prospective studies. PLoS One, 2013. 8(2): p. e56540.

12. Prado, C.M., et al., Prevalence and clinical implications of sarcopenic obesity in patients with solid tumours of the respiratory and gastrointestinal tracts: a population-based study. Lancet Oncol, 2008. 9(7): p. 629-35.

13. Rahbari, N.N. and J. Weitz, Hyaluronic acid as a marker of sinusoidal obstruction syndrome after oxaliplatin-based chemotherapy for colorectal liver metastases: don't forget the tumor. Ann Surg Oncol, 2013. 20(5): p. 1405-7.

14. Qian, N.S., et al., Comprehensive application of modern technologies in precise liver resection. Hepatobiliary Pancreat Dis Int, 2013. 12(3): p. 244-50.

15. de Santibanes, E. and P.A. Clavien, Playing Play-Doh to prevent postoperative liver failure: the "ALPPS" approach. Ann Surg, 2012. 255(3): p. 415-7.

16. Aloia, T.A. and J.N. Vauthey, Associating liver partition and portal vein ligation for staged hepatectomy (ALPPS): what is gained and what is lost? Ann Surg, 2012. 256(3): p. e9; author reply e16-9.

17. Nagino, M., et al., Hepatectomy with simultaneous resection of the portal vein and hepatic artery for advanced perihilar cholangiocarcinoma: an audit of 50 consecutive cases. Ann Surg, 2010. 252(1): p. 115-23.

18. Neuhaus, P., et al., Oncological superiority of hilar en bloc resection for the treatment of hilar cholangiocarcinoma. Ann Surg Oncol, 2012. 19(5): p. 1602-8.

19. Nagino, M., et al., "Anatomic" right hepatic trisectionectomy (extended right hepatectomy) with caudate lobectomy for hilar cholangiocarcinoma. Ann Surg, 2006. 243(1): p. 28-32.

20. Neumann, U.P. and C.H. Dejong, Split decision. Br J Surg, 2013. 100(3): p. 310-2.

21. Schnitzbauer, A.A., et al., Right portal vein ligation combined with in situ splitting induces rapid left lateral liver lobe hypertrophy enabling 2-staged extended right hepatic resection in small-for-size settings. Ann Surg, 2012. 255(3): p. 405-14.

22. Knoefel, W.T., et al., In situ liver transection with portal vein ligation for rapid growth of the future liver remnant 
in two-stage liver resection. Br J Surg, 2013. 100(3): p. 388-94.

23. McCulloch, P., et al., No surgical innovation without evaluation: the IDEAL recommendations. Lancet, 2009. 374(9695): p. 1105-12.

24. Zwarenstein, M., et al., Improving the reporting of pragmatic trials: an extension of the CONSORT statement. BMJ, 2008. 337: p. a2390.

25. van den Broek, M.A., et al., Feasibility of randomized controlled trials in liver surgery using surgery-related mortality or morbidity as endpoint. Br J Surg, 2009. 96(9): p. 1005-14.

26. Rahbari, N.N., et al., Post-hepatectomy haemorrhage: a definition and grading by the International Study Group of Liver Surgery (ISGLS). HPB (Oxford), 2011. 13(8): p. 528-35. 27. Rahbari, N.N., et al., Posthepatectomy liver failure: a definition and grading by the International Study Group of Liver Surgery (ISGLS). Surgery, 2011. 149(5): p. 713-24.

28. Koch, M., et al., Bile leakage after hepatobiliary and pancreatic surgery: a definition and grading of severity by the International Study Group of Liver Surgery. Surgery, 2011. 149(5): p. 680-8.

29. Strasberg, S.M., Nomenclature of hepatic anatomy and resections: a review of the Brisbane 2000 system. J Hepatobiliary Pancreat Surg, 2005. 12(5): p. 351-5.

30. van den Broek, M.A., et al., Development of a composite endpoint for randomized controlled trials in liver surgery. Br J Surg, 2011. 98(8): p. 1138-45.

31. de Boer, M.T., et al., Fibrin sealant for prevention of resection surface-related complications after liver resection: a randomized controlled trial. Ann Surg, 2012. 256(2): p. 229-34.

32. Mpabanzi, L., et al., Surrogate endpoints in liver surgery related trials: a systematic review of the literature. HPB (Oxford), 2013. 15(5): p. 327-36.

33. Buell, J.F., et al., The international position on laparoscopic liver surgery: The Louisville Statement, 2008. Ann Surg, 2009. 250(5): p. 825-30.

34. Maessen, J.M., et al., Length of stay: an inappropriate readout of the success of enhanced recovery programs. World J Surg, 2008. 32(6): p. 971-5.

35. Fiore, J.F., Jr., et al., Criteria to determine readiness for hospital discharge following colorectal surgery: an international consensus using the Delphi technique. Dis Colon Rectum, 2012. 55(4): p. 416-23.

36. Fiore, J.F., et al., Time to readiness for discharge is a valid and reliable measure of short-term recovery after colorectal surgery. World J Surg, 2013.

37. Junejo, M.A., et al., Cardiopulmonary exercise testing for preoperative risk assessment before hepatic resection. Br J Surg, 2012. 99(8): p. 1097-104.

38. Gomez, D., et al., Steatosis predicts postoperative morbidity following hepatic resection for colorectal metastasis. Br J Surg, 2007. 94(11): p. 1395-402.

39. Hamady, Z.Z., et al., Fatty liver disease as a predictor of local recurrence following resection of colorectal liver metastases. Br J Surg, 2013. 100(6): p. 820-6.

40. Young, A.L., et al., Role of quantification of hepatic steatosis and future remnant volume in predicting hepatic dysfunction and complications after liver resection for colorectal metastases: a pilot study. HPB (Oxford), 2012. 14(3): p. 194-200.

41. Cauchy, F., et al., Surgical treatment of hepatocellular carcinoma associated with the metabolic syndrome. $\mathrm{Br} J$ Surg, 2013. 100(1): p. 113-21.

42. Marsman, H.A., et al., Hepatic regeneration and functional recovery following partial liver resection in an experimental model of hepatic steatosis treated with omega-3 fatty acids. Br J Surg, 2013. 100(5): p. 674-83.

43. Goshima, S., et al., Gd-EOB-DTPA-enhanced MR imaging: prediction of hepatic fibrosis stages using liver contrast enhancement index and liver-to-spleen volumetric ratio. J Magn Reson Imaging, 2012. 36(5): p. 1148-53.

44. Peng, P.D., et al., Sarcopenia negatively impacts shortterm outcomes in patients undergoing hepatic resection for colorectal liver metastasis. HPB (Oxford), 2011. 13(7): p. 439-46.

45. Dello, S.A., et al., Sarcopenia negatively affects preoperative total functional liver volume in patients undergoing liver resection. HPB (Oxford), 2013. 15(3): p. 165-9.

46. Fearon, K.C., et al., Patient optimization for gastrointestinal cancer surgery. Br J Surg, 2013. 100(1): p. 15-27. 47. Tan, B.H., et al., Sarcopenia in an overweight or obese patient is an adverse prognostic factor in pancreatic can- 
cer. Clin Cancer Res, 2009. 15(22): p. 6973-9.

48. Fearon, K.C., Cancer cachexia and fat-muscle physiology. N Engl J Med, 2011. 365(6): p. 565-7.

49. Wigmore, S.J., et al., Virtual hepatic resection using three-dimensional reconstruction of helical computed tomography angioportograms. Ann Surg, 2001. 233(2): p. 221-6.

50. Imamura, H., et al., Assessment of hepatic reserve for indication of hepatic resection: decision tree incorporating indocyanine green test. J Hepatobiliary Pancreat Surg, 2005. 12(1): p. 16-22.

51. Clavien, P.A., et al., Strategies for safer liver surgery and partial liver transplantation. N Engl J Med, 2007. 356(15): p. $1545-59$.

52. Makuuchi, M., et al., Surgery for small liver cancers. Semin Surg Oncol, 1993. 9(4): p. 298-304.

53. Lock, J.F., et al., Function and volume recovery after partial hepatectomy: influence of preoperative liver function, residual liver volume, and obesity. Langenbecks Arch Surg, 2012. 397(8): p. 1297-304.

54. Stockmann, M., et al., The LiMAx test: a new liver function test for predicting postoperative outcome in liver surgery. HPB (Oxford), 2010. 12(2): p. 139-46.

55. de Graaf, W., et al., Assessment of future remnant liver function using hepatobiliary scintigraphy in patients undergoing major liver resection. J Gastrointest Surg, 2010. 14(2): p. 369-78.

56. van den Broek, M.A., et al., Hyaluronic acid as a marker of hepatic sinusoidal obstruction syndrome secondary to oxaliplatin-based chemotherapy in patients with colorectal liver metastases. Ann Surg Oncol, 2013. 20(5): p. $1462-9$

57. Vreuls, C.P., et al., Hepatic sinusoidal obstruction syndrome (SOS) reduces the effect of oxaliplatin in colorectal liver metastases. Histopathology, 2012. 61(2): p. 314-8.

58. Dello, S.A., et al., Systematic review of ophthalmate as a novel biomarker of hepatic glutathione depletion. Clin Nutr, 2013. 32(3): p. 325-30.

59. Birkmeyer, J.D., et al., Hospital volume and surgical mortality in the United States. N Engl J Med, 2002. 346(15): p. 1128-37.

60. Finks, J.F., N.H. Osborne, and J.D. Birkmeyer, Trends in hospital volume and operative mortality for high-risk surgery. N Engl J Med, 2011. 364(22): p. 2128-37.

61. Birkmeyer, J.D., et al., Surgeon volume and operative mortality in the United States. N Engl J Med, 2003. 349(22): p. 2117-27.

62. de Vries, E.N., et al., Effect of a comprehensive surgical safety system on patient outcomes. N Engl J Med, 2010. 363(20): p. 1928-37.

63. Urbach, D.R. and N.N. Baxter, Reducing variation in surgical care. BMJ, 2005. 330(7505): p. 1401-2.

64. Wong-Lun-Hing, E.M., et al., A survey in the hepatopancreatobiliary community on ways to enhance patient recovery. HPB (Oxford), 2012. 14(12): p. 818-27.

65. Strasberg, S.M., D.C. Linehan, and W.G. Hawkins, The accordion severity grading system of surgical complications. Ann Surg, 2009. 250(2): p. 177-86.

66. Satava, R.M., Identification and reduction of surgical error using simulation. Minim Invasive Ther Allied Technol, 2005. 14(4): p. 257-61.

67. Stoot, J.H., et al., The effect of a multimodal fast-track programme on outcomes in laparoscopic liver surgery: a multicentre pilot study. HPB (Oxford), 2009. 112(2): p. 140-44.

68. van Dam, R.M., et al., Open versus laparoscopic left lateral hepatic sectionectomy within an enhanced recovery ERAS(R) programme (ORANGE II-trial): study protocol for a randomised controlled trial. Trials, 2012. 13: p. 54.

69. Young, e.a., Variation in referral practice for patients with colorectal cancer liver metastases. Br J Surg, 2013.

70. Jones, R.P., et al., Effect of specialist decision-making on treatment strategies for colorectal liver metastases. $\mathrm{Br}$ J Surg, 2012. 99(9): p. 1263-9.

71. Vigano, L., et al., Progression while receiving preoperative chemotherapy should not be an absolute contraindication to liver resection for colorectal metastases. Ann Surg Oncol, 2012. 19(9): p. 2786-96.

72. de Haas, R.J., et al., R1 resection by necessity for colorectal liver metastases: is it still a contraindication to surgery? Ann Surg, 2008. 248(4): p. 626-37.

73. Maas, M., et al., Wait-and-see policy for clinical complete responders after chemoradiation for rectal cancer. J Clin Oncol, 2011. 29(35): p. 4633-40. 


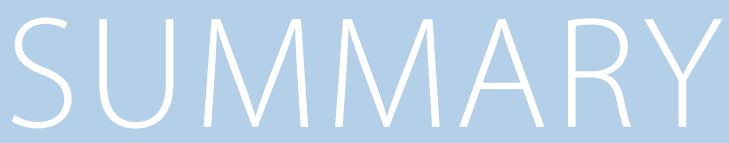

This thesis focuses on the clinical challenges of modern liver surgery. Novel strategies for primary and secondary liver tumours are studied and suggestions are done to optimize clinical care, to make research and clinical results comparable and to make the design of future trials feasible. 
Liver resection for malignant tumours is the only curative strategy, among anti-tumour therapies like systemic chemotherapy, radiofrequency- or microwave ablation, arterial chemoembolization and stereotactic radiotherapy. Annually, in the Netherlands, more than 2000 patients with a variety of malignant or symptomatic benign tumours are potential candidates for liver resection. Primary liver-, bile duct- or gallbladder-cancer or secondary colorectal cancer-, kidney cancer-, breast cancer- and neuro endocrine tumour-metastases as well as symptomatic liver adenomas or hemangioma's constitute the main indications for these resections.

Traditionally liver resection was considered dangerous. Improvements in referral patterns, surgical techniques and perioperative care together with multidisciplinary patient selection, centralization of care and international knowledge exchange have made liver resection reasonably safe. Complications after liver resection occur in $30-40 \%$ of patients and are reasonably predictable, with haemorrhagic complications occurring predominantly during surgery or in the early postoperative phase, and biliary complications, intra-abdominal abscess or liver failure in the later postoperative phase. Complications often require surgical, endoscopic, or radiological interventions but are usually well tolerated by patients and only influencing recovery in the postoperative course temporarily.

The current focus in liver surgery is how to extend the indication criteria to make more patients eligible for resection, how to reduce the risk and how to improve short- and long-term outcome.

\section{PART 1 - ADVANCING THE LIMITS OF LIVER SURGERY}

In part 1 of this thesis the outcome of extending the indication criteria for resection and reversing the treatment sequence by resecting the liver metastases before the colorectal cancer primary is discussed.

Formerly, only patients with a maximum of 3 colorectal cancer liver metastases, located peripherally at one side of the liver, with an anticipated resection margin greater than 10 millimetre and without signs of extra hepatic disease were considered eligible for partial liver resection. Today, tumour clearance with a minimal resection margin of $1 \mathrm{~mm}$ is sufficient to achieve a significant survival benefit. Consequently, postoperative remnant liver function has become the most important determinant of resectability.

The study in chapter 2 assessed 298 patients who underwent liver resection for CRCLM between 1991 and 2010 at the Maastricht University Medical Centre. 169 patients were included in the limited indications group and 129 patients in the extended indications group.

It was observed that extending the indications for liver resection of colorectal cancer liver metastases renders more patients eligible for resection but also showed that extending the 
indications has its consequences for patients. There were no significant differences in blood loss during surgery, length of hospital stay, number of readmissions and 90-day postoperative mortality rate between both groups. However, in the extended indications group median operation time was significantly longer, major complications occurred more often and the microscopic radical resection (R0) rate was lower. Over time $56 \%$ of microscopically not radical (R1) resected patients presented recurrent systemic metastases but interestingly, in only $17 \%$ at the former $\mathrm{R} 1$ resection surface of the liver. Patients in the limited indications group had a 5 year overall survival rate of $60.5 \%$ the extended indications criteria group had a 5 -year survival of $33.2 \%$. 10-year overall survival rates were $35.5 \%$ and $15.8 \%$, respectively.

As others have provided data to suggest these patients have a good quality of life, it seems justified to be creative in future strategies to increase the resection rate in patients with colorectal cancer liver metastases. It may be a suggestion to combine different treatment modalities that aim to remove or destroy metastases while functional liver capacity is maintained or enhanced.

The study in chapter 3 assessed feasibility and outcome of a liver first strategy in 22 patients who underwent liver resection for CRCLM mainly of rectal cancer between 2005 and 2010 at the Maastricht University Medical Centre. The classic approach for patients with synchronous colorectal cancer liver metastases consists of resection of the primary tumour, followed by optional adjuvant chemotherapy and eventually succeeded by liver surgery. Major disadvantages of this treatment sequence are serious hepatoxicity, radiologically but not histologically vanishing liver lesions or the potential progression of liver metastases beyond resectability. Especially when morbidity associated with colorectal surgery delays treatment. Advantage of the reversed sequence of therapy is that distant metastases are the main cause of death and that it is therefore important to eliminate these lesions first. With a liver-first approach, the treatment of the CRCLM is by no means interrupted by possible complications after resection of the primary tumour. Another advantage could be that administration of pre-operative chemotherapy provides a chance to evaluate a response and thereby defines the tumour biology of the CRCLM. Moreover the liver first strategy provides a certain window during which possibly latently present extra hepatic metastases have a chance to declare themselves.

In the study 18 patients (81.8\%) completed the liver first treatment plan for liver and primary tumours. Importantly, the interval between the operations for the hepatic and primary disease was similar for patients who did and patients who did not develop complications after liver surgery. The remaining patients deviated from the protocol as result of the development of unresectable extra hepatic metastases during the course of their treatment (i.e. under chemotherapy). This supports the potential benefit of the surgery-free interval as patients can be 'saved' an operation in the case of disease progression. Especially when the 
primary tumour requires a neo-adjuvant treatment strategy that provides a window for the liver-first approach, this sequence of therapy should be considered in the multidisciplinary individualized treatment plan of these patients.

Finally, the observations in this study may be an argument to start the treatment sequence of patients with synchronous CRCLM with 3 cycles of systemic chemotherapy, followed by liver surgery and the last 3 cycles of chemotherapy thereafter. Colorectal surgery is performed at the end of the treatment sequence and only in patients that are really likely to benefit.

\section{REDUCING THE RISKS OF LIVER SURGERY}

In part 2 of this thesis we describe virtual liver resection and liver volumetry with 3 different image analysis systems. Accurate preoperative risk assessment to determine whether a patient can undergo major or extended liver resection remains the Holy Grail of liver surgery. In so far as volume equates to function, and affordable accurate systems to predict future liver remnant function lack, assessment of liver volume, and particularly the volume of the future liver remnant, is critical. Especially in patients with compromised liver function due to neoadjuvant chemotherapy, cirrhosis or steatosis and also for patients scheduled for an extended liver resection.

Liver volumes can be accurately calculated from computed tomography (CT) or magnetic resonance imaging (MRI) scans but liver volumetry software is usually linked to the scanner systems and requires an experienced radiologist. In addition, the intended operation should be known to predict the future remnant liver volume accurately and this requires the expertise of a liver surgeon. Due to lack of affordable, user-friendly, time efficient and easily accessible instruments liver volumetry is often not easily available to the multidisciplinary team advising on the indication for and possibility of resectional liver surgery. However, the recent development of downloadable open source or governmentally funded image analysis software and the Digital Imaging and Communications in Medicine (DICOM) standard have enabled volumetry on a stand-alone or network-attached personal computer remote from the radiology hardware (CT or MRI scanner).

In chapter 4 we describe the use and test the accuracy of the freely downloadable image analysis software ImageJ for hepatic CT volumetry in 15 patients undergoing major hepatectomy for colorectal cancer metastases. ImageJ has been developed at the National Institute of Health (NIH) to assist in clinical image analysis. In the study we showed a strong correlation between resected liver weight and preoperatively CT-measured liver volume. The study proofed that accurate $\mathrm{CT}$ volumetric analysis can be performed by a surgeon on a personal computer independent of the support of a radiologist. However, volumetry with ImageJ is laborious. Manually outlining the liver takes 50 seconds per CT slice and analysis 
of an entire liver requires on average 25 to 28 minutes. By raising the slice-thickness to 1 $\mathrm{cm}$ the time required to perform the volumetry was reduced and accuracy not jeopardized.

Recently, the more sophisticated and user-friendly, freely downloadable open source software OsiriX has become available. A team working at UCLA, Los Angeles, USA and Le Tour Hospital, Geneva, Switzerland has developed the system.

In chapter 5 we describe the use of OsiriX CT volumetry and test the accuracy in 25 patients undergoing liver resection. Results are compared to liver CT volumetry performed by a radiologist using classical radiological software (iNtuition, Terra Recon) linked to the Maastricht UMC CT-scanner system.

In the study OsiriX as well as iNtuition showed a strong correlation between resected liver weight and preoperatively CT-measured liver volume. It proofed that accurate CT volumetric analysis can be performed with both systems. The mean time needed to analyse the total liver volume, tumour volume, and resection volume with OsiriX was 19 minutes. This is slightly faster than the time reported for ImageJ and can be explained by the semiautomatic selection of the liver and regions of interest with OsiriX.

In the study, we also discovered that volumes calculated by iNtuition were inexplicably altered when the Hounsfield window level was changed, e.g., from abdominal to liver window. This may have resulted in differences in volumetric results between the two packages as well as between patients. Moreover, this casts some doubt as to whether iNtuition should be considered the "gold standard" for clinical use. As consequence Terra Recon, the company that makes iNtuition, started to work on a solution to address this undesired phenomenon and has developed a dedicated liver tissue template. Future research will be needed to determine whether that new template is more accurate in assessing liver volume.

In chapter 6 we compare the accuracy in CT liver volumetry with ImageJ, OsiriX and iNtuition in 15 patients undergoing right hemi-hepatectomy. There was a strong correlation with the weights of the resected hemi-livers and between the volumes measured with radiology software iNtuition and the volumes measured with ImageJ and OsiriX. This shows that ImageJ and OsiriX, even if operated by non-radiologists, are at least as appropriate for CT volumetry of the liver as iNtuition professional radiology software. Moreover, OsiriX has a significant advantage over ImageJ because it has an integrated picture archiving and communication system (PACS system) in which the CT scan and volumetry data of all examined patients are automatically stored. 
IMPROVING THE OUTCOME OF LIVER SURGERY: MODIFY OPERATIONS AND MODIFY PERIOPERATIVE CARE

Perioperative care in patients undergoing liver resection is still heavily influenced by dogma and tradition. Within a traditional perioperative care pathway median hospital stay following liver resection ranges from 8 to 14 days.

Multimodal perioperative care protocols commonly referred to as fast track or enhanced recovery programs manage expectations, target clinical factors that delay postoperative recovery as pain, gut dysfunction and immobility, and combine a series of interventions to reduce perioperative stress and organ dysfunction. Such programs, allow well-informed patients to participate actively in the recovery process and have been shown to accelerate postoperative recovery and consequently reduce length of hospital stay.

In chapter 7 we describe the implementation study of the evidence-based multimodal Enhanced Recovery After Surgery (ERAS) liver program, newly developed by the liver units of the international collaborative ERAS group in Maastricht, Edinburgh and Tromsø. The enhanced recovery protocol was studied prospectively in a consecutive series of 61 patients undergoing liver resection at the liver unit of Maastricht University Medical Centre, The Netherlands or the liver unit of the Royal Infirmary of Edinburgh, UK. Outcomes were compared with those in a consecutive series of 100 patients who underwent liver resection in both units before the implementation, when a more traditional care pathway was still in use.

The study showed that use of an enhanced recovery program in liver resection accelerated postoperative recovery and led to a significantly shorter hospital stay. Most patients managed according to the ERAS protocol were able to drink fluids within $4 \mathrm{~h}$ of liver resection, were eating normal food on the day after surgery and were discharged on the fifth postoperative day. Moreover, the program appeared to be safe since there were no significant differences in rates of readmission, morbidity and mortality between ERAS and control groups.

Another trend is the growing preference of laparoscopic liver resection over open resection. Laparoscopic liver resection may have many benefits over conventional open liver resection. Multiple publications in the last 15 years present data of more than 4000 patients and are consistently showing that these patients suffer from less pain, recover quicker and have a shorter length of hospital stay. However, all these outcomes, among others, are probably prone to bias as they have never been studied in a randomized controlled trial comparing open en laparoscopic liver resection.

It is only recently that the surgical community has accepted the idea that both strategies to accelerate recovery may be synergistic. The laparoscopic approach is just another way to reduce the surgical insult to the patient and can be seen as a core element of an ERAS 
program.

In chapter 8 we describe a retrospective, multicentre study assessing the effect of an ERAS program in patients undergoing laparoscopic minor liver resection. 26 patients were treated by laparoscopic liver resection during a 5 -year period. Outcomes of thirteen patients undergoing laparoscopic liver resection within the ERAS program in the Maastricht UMC were compared with thirteen patients undergoing laparoscopic liver resection in a traditional perioperative care pathway at Maastricht UMC $(n=3)$, AMC Amsterdam ( $n=4)$, UMC Utrecht $(n=2)$ and UMC Groningen $(n=4)$. Although small, the study suggests that a multimodal enhanced recovery program in laparoscopic liver surgery is feasible, safe and may lead to accelerated functional recovery and a reduction in hospital length of stay

Today, it remains unclear which components of reduced hospital length of stay result from the introduction of a formal care pathway rather than the enhanced recovery elements of that pathway.

The challenge is to assess, one by one, the core elements of recovery programs in adequately powered and well-designed multicentre trials.

Also, more and more it is being recognized that hospital length of stay, the most used outcome parameter in studies assessing the value of the laparoscopic technique or enhanced recovery programs, is not only dependent on recovery but also very much influenced by patient expectations and discharge planning of hospital staff. Time to functional recovery instead of hospital length of stay may be a better outcome parameter for future trials. A patient is fully functionally recovered or medically ready for discharge when the following five criteria are satisfied: Adequate pain control with oral analgesics, mobilized at preoperative level, tolerance of solid food, normal or decreasing bilirubin and INR of at least $80 \%$ of the normal value, no intravenous fluids

In chapter 9 we describe the randomized controlled ORANGE trial (Optimized Recovery with Accelerated Nutrition and Gastrointestinal Enhancement). In that trial we assessed within an ERAS protocol, whether postoperative bowel stimulation with magnesium hydroxide and/ or Oral Nutritional Support (ONS) are effective in promoting early return of gastrointestinal function and overall postoperative recovery.

68 patients undergoing liver resection at the liver unit of the Royal Infirmary of Edinburgh, UK or the liver unit of Maastricht University Medical Centre, The Netherlands were randomized. Most patients in the trial recommenced oral fluid on the day of surgery, started eating the following day, were mobile by the third day and achieved discharge criteria on the fourth day. Laxatives significantly decreased the time to passage of stool after liver surgery. Calorie intake of the group randomized to ONS was not different from that of patients 
who did not receive ONS, suggesting that ONS in the early postoperative phase suppressed normal food intake to the point at which there was no net benefit. Median hospital stay was 6 days, which is significantly shorter than the 8-14 days reported in other centres following traditional perioperative care. Functional recovery was achieved a median of 4 days after surgery, a median of 2 days before discharge from hospital.

In chapter 10 we present the results of an international survey representing 161 hepatopancreatobiliary (HPB) centres worldwide. It demonstrates that adoption of enhanced recovery programmes in liver surgery is limited and warrants greater attention in HPB community. The survey also demonstrates that the majority of HPB centres perform laparoscopic liver surgery although the number of laparoscopic resections carried out in the majority of centres is low. More than half of HPB centres consider a randomized controlled trial comparing outcomes in open and laparoscopic liver surgery prior to the further adoption of laparoscopic liver surgery necessary.

In chapter 11 we present the study protocol of 2 actively accruing international multicentre randomized controlled trials, comparing open and laparoscopic left lateral sectionectomy (ORANGE II) or hemi-hepatectomy (ORANGE II Plus) within an ERAS programme.

The primary endpoint of both the ORANGE II as the ORANGE II plus trial is time to functional recovery. Secondary endpoints are postoperative hospital length of stay, readmission percentage, a composite endpoint of liver surgery specific morbidity, quality of life, body image and cosmetic result, hospital and societal costs over 1 year, long-term incidence of incisional hernias and overall survival in oncologic patients. In both trials it is assumed that time to functional recovery after laparoscopic resection compared to open resection can be reduced by two days. Sample size for the ORANGE II trial has been calculated at 55 patients in each randomization arm. Sample size for the ORANGE II Plus trial has been calculated at 125 patients in each randomization arm.

The question remains whether an RCT is necessary to prove that laparoscopy should be accepted as the preferred method to perform liver resection. Randomization of patients undergoing open or laparoscopic liver resection is not without difficulties. Surgeons and patients in experienced centres will be reluctant to randomize patients because of the absence of clinical and patient equipoise for the laparoscopic resection. Also inexperienced surgeons may be reluctant since they want to embark on laparoscopic liver resection and need the numbers to get through the learning curve. There are even experienced surgeons claiming randomizing patients to be unethical since, in their opinion, enough outcome data have already been published.

However, in most reported trials or systematic reviews aiming at earlier recovery, less pain or 
a reduction in LOS after laparoscopic liver resection, type of resection and/or perioperative management were not standardized. Mostly it is unclear which definitions are used for specific outcome parameters and how they were registered.

Moreover it gets more and more recognized that hospital length of stay is an unreliable outcome parameter since it is to dependent of patient's expectations, planning, culture, hospital logistics and reimbursements.

In the ORANGE II trials, the ERAS enhanced recovery protocol provides the perioperative standardization needed. The primary outcome parameter functional recovery representing medically being ready for discharge, is well measurable and based on hard clinical recovery criteria. The use of experienced proctor surgeons in centres with limited experience in laparoscopic liver surgery should eliminate learning curve influences on the outcome. The economic evaluation will include a cost-utility analysis from hospital and a Dutch societal perspective and will provide data on costs per quality adjusted life year (QALY).

The trial design combining an RCT and a prospective registry will improve overall power and strengthen the external validity and generalizability of study results.

\section{GENERAL DISCUSSION AND PERSPECTIVES}

Over the past century, the number of liver resections performed globally has increased immensely. It is only by the continuous striving for improvement in patient care that this has all become possible. Staged hepatectomies, repeat liver resections, two stage liver resections after unilateral portal vein ligation, liver resections after preoperative portal vein embolization and the liver first approach have all seen the light. Combinations with induction chemotherapy, needle radiofrequency ablation, trans arterial embolization, in situ liver perfusion and Gamma knife or stereotactic radiotherapy have been introduced. All of these have remarkably increased the surgical armamentarium to combat primary or secondary liver tumours.

The problem with most of the new surgical procedures is that they are introduced into the field of liver surgery in a rather uncontrolled way. None of the aforementioned procedures have been subjected to proper testing in randomized controlled trials and may have exposed patients to unnecessary risks. It is evident that this requires change. A major problem is that trials are difficult to perform in surgery. Good Clinical Practice regulations are cumbersome and the incidence of some of the most important outcomes in liver surgery, such as bile leak, liver failure and mortality is too low to make a trial realistic in terms of sufficient patient enrolment or trial duration. To help to solve this problem we developed a composite, liver specific endpoint, which consists of a combination of clinical relevant liver specific individual single endpoints, with each a rather low incidence following liver resections. If combined, the incidence of the liver specific combined endpoint increases and this facilitates 
the feasibility of conducting a randomized trial requiring a considerable smaller sample size. Moreover, consistent use of this liver surgery specific CEP may improve comparability of trials and the quality of future meta-analyses.

The way patients are prepared to undergo often a series of interventions as well as the organization of care after such interventions is key in determining outcome.

In the Maastricht University Medical Centre, we gained considerable experience with implementation of best practice peri-operative care for patients: the so-called Enhanced Recovery After Surgery or fast-track programs. This peri-operative care program leads to faster recovery due to optimal pain management, early feeding, less tubes and drains, avoidance of insulin resistance and active mobilization after surgery. Since as a consequence, patients leave the hospital quicker and in a better physical condition this appears to reduce costs and may allow for an earlier start of adjuvant chemotherapy in patients that underwent cancer surgery.

Recently we have shown that even today it still takes more than a decade to implement this knowledge into global HPB surgical practice. Collaborative initiatives, as the Dutch Liver Working Group, defining datasets for clinical multicentre trials and continuous national audits of the Dutch Institute of Clinical Auditing and the international Enhanced Recovery after Surgery group, may help to accelerate the implementation of trial outcomes. A prerequisite for all trials and audits to be unconditionally comparable is the use of globally accepted well-defined outcomes and classification systems. The Accordion ${ }^{\oplus}$, modified Clavien-Dindo-Strasberg, classification system of postoperative surgical complications is the best-defined classification system but disregards intraoperative adverse events. The Satava-classification system for intraoperative adverse events, may be the most suitable to fill that deficit

Until recently but also still today, liver surgery was not offered to many eligible patients. This was not based on objective criteria and it is highly likely that many patients have been denied operations that could have cured them. Ignorance on oncological outcome by non-specialized teams and lack of criteria to measure patients' resilience to surgery and its complications are responsible. Clearly, each patient with colorectal cancer metastases, but probably also every patient with a liver tumour, should be discussed at a specialized liver and colorectal cancer multidisciplinary team meeting. Only in this way will patients have access to optimal expertise and treatment. Therefore, the organization of care should focus on avoiding complications but even more important on how to rescue patients from these often life threatening events, if they occur. A specialized multidisciplinary hepatobiliairy team looking after those patients on a $24 / 7$ basis underlines the importance of centralization in high-volume hospitals. Moreover, assessment of fitness to withstand the stress of the potential complications needs to be developed and should be based on objective criteria. Evaluation of the patient by preoperative cardiopulmonary exercise testing and CT-based 
volumetric measurement of skeletal muscle mass to assess nutritional status may help to select patients at higher risk for liver surgery.

Just recently individualized patient care and patient safety have been put increasingly on the agenda. The use of structured informed consent, check lists and sign in and sign out procedures as well as stopping moments in surgical procedures has contributed strongly to patient safety. This will no doubt be reflected in patient reported outcome assessment, as suggested by patient societies, to measure outcome. Individualized patient care, although more and more possible, may conflict with the societal strive to offer cost effective treatments. Off course, the treatment proposed to the patients should be tailored and individualized, whereby the acceptance of that proposal by the patients is made by a shared decision of both patient and caregiver. It has become clear that patients often have strong preferences, and their preferences do not always coincide with the preferences of their doctors, the guidelines or the society. In this context it is important to realize that medical specialists, society and patients judge the benefit of a treatment differently. In exploring the limits of resectional liver surgery many members of a multidisciplinary team emphasize what can go wrong while most patients are prepared to take a calculated risk based on what can go well. 


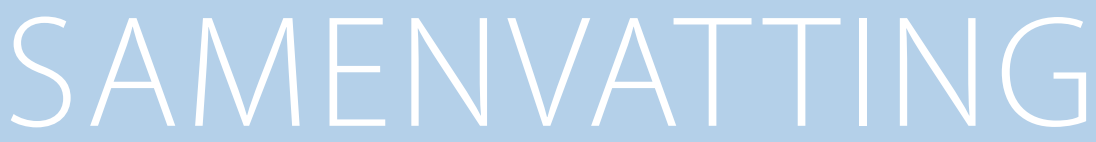

Dit proefschrift richt zich op de klinische uitdagingen van de moderne lever chirurgie. Nieuwe strategieën voor de behandeling van primaire en secundaire levertumoren worden bestudeerd en suggesties worden gedaan om de klinische zorg te optimaliseren, onderzoek en klinische resultaten vergelijkbaar te maken en het ontwerpen van toekomstige onderzoeken mogelijk te maken. 
Leverresectie voor kwaadaardige tumoren is, onder anti-tumor therapieën zoals systemische chemotherapie, radiofrequente-of microgolf-ablatie, arteriële chemo-embolisatie en stereotactische radiotherapie, de enige curatieve strategie. In Nederland zijn jaarlijks meer dan 2000 patiënten, met diverse kwaadaardige of symptomatische goedaardige tumoren, potentieel kandidaat voor leverresectie. De belangrijkste indicaties voor deze leverresecties bestaan uit lever-, galweg-of galblaas-kanker of uitzaaiingen van darmkanker, nierkanker, borstkanker en neuro endocriene tumoren. Daarnaast wordt een beperkt deel van de resecties verricht voor symptomatische lever adenomen of hemangiomen.

In het verleden werd leverresectie als gevaarlijk beschouwd. Verbeteringen in chirurgische technieken en perioperatieve zorg hebben samen met multidisciplinaire selectie van patiënten, centralisatie van zorg en internationale kennisuitwisseling, leverresectie redelijk veilig gemaakt. Complicaties na leverresectie komen bij 30-40\% van de patiënten voor en zijn redelijk voorspelbaar. Bloedingscomplicaties treden overwegend op tijdens de operatie of in de vroege postoperatieve fase. Galweg complicaties, intra-abdominale abcessen of leverfalen in de latere postoperatieve fase. Complicaties vereisen vaak chirurgische, endoscopische of radiologische interventies, maar worden doorgaans door patiënten goed verdragen en beïnvloeden slechts tijdelijk het herstel proces.

De huidige focus in de lever chirurgie is hoe de indicatie criteria uit te breiden zodat meer patiënten in aanmerking komen voor resectie, hoe het risico te verminderen en hoe korte en lange termijn resultaten te verbeteren.

\section{DEEL 1 - DE GRENZEN IN DE LEVER CHIRURGIE}

In deel 1 van dit proefschrift wordt het resultaat van het uitbreiden van de indicatie criteria voor lever resectie en het omkeren van de behandelvolgorde bij patiënten met uitzaaiingen van darm- of endeldarm-kanker besproken.

Tot enkele jaren geleden kwamen alleen patiënten met maximaal 3 uitzaaiingen, perifeer gelegen in één zijde van de lever, met een verwachte resectie marge groter dan $10 \mathrm{~mm}$ en zonder aanwijzingen op uitzaaiingen buiten de lever in aanmerking voor partiële leverresectie. Tegenwoordig kunnen meerdere uitzaaiingen, ook buiten de lever worden verwijderd en is een minimale resectie marge van $1 \mathrm{~mm}$ voldoende om een significant overlevingsvoordeel te bereiken. Functie van de restlever na leverresectie is nu de belangrijkste determinant van resectabiliteit geworden.

De studie in hoofdstuk 2 onderzocht 298 patiënten die in het Maastricht UMC tussen 1991 en 2010 een partiële leverresectie voor colorectale leveruitzaaiingen ondergingen. 169 patiënten zaten in de groep met beperkte indicatie criteria en 129 patiënten in de groep met uitgebreide indicatie criteria. 
De studie liet zien dat door het uitbreiden van de indicatie criteria meer patiënten in aanmerking komen voor leverresectie maar dat het uitbreiden van de criteria ook zijn gevolgen heeft voor patiënten. Er waren geen significante verschillen in bloedverlies tijdens de operatie, de duur van verblijf in het ziekenhuis, het aantal heropnames en 90 dagen postoperatieve sterfte tussen beide groepen. Echter, in de uitgebreide indicatie groep was de mediane operatietijd aanzienlijk langer, kwamen grote complicaties vaker voor en het microscopische radicale resectie (R0) percentage was lager. Na verloop van tijd kreeg 56\% van patiënten met een microscopisch niet radicale resectie (R1) recidief uitzaaiingen, maar slechts $17 \%$ op de plaats van het oude R1 resectievlak. Patiënten met beperkte indicatie criteria hadden een 5 -jaars overleving van $60,5 \%$ met de uitgebreide indicatie criteria een 5 -jaars overleving van $33,2 \%$. De 10 -jaars overleving was respectievelijk $35,5 \%$ en $15,8 \%$.

Ook andere onderzoeken hebben laten zien dat deze patiënten een goede kwaliteit van leven hebben. Het lijkt daarom gerechtvaardigd om creatief te zijn in strategieën die het toekomstige resectie percentage bij patiënten met colorectale uitzaaiingen in de lever kunnen verhogen. Het kan zinnig zijn om verschillende behandelingen te combineren die alle gericht zijn op verwijderen of vernietigen van uitzaaiingen, terwijl de functionele lever capaciteit wordt gehandhaafd of vergroot.

De studie in hoofdstuk 3 onderzocht de haalbaarheid en het klinisch resultaat van een levereerst strategie bij 22 patiënten die in het Maastricht UMC tussen 2005 en 2010 een leverresectie ondergingen voor colorectale leveruitzaaiingen van voornamelijk endeldarmkanker. De klassieke benadering voor patiënten met synchrone colorectale leveruitzaaiingen omvat resectie van de primaire tumor, optioneel gevolgd door aanvullende chemotherapie en uiteindelijk gevolgd door leverchirurgie. Nadelen van deze behandelingsvolgorde zijn leverschade door de chemotherapie, het radiologisch maar niet histologisch verdwijnen van resectabele leveruitzaaiingen of mogelijke progressie van de uitzaaiingen tot voorbij de resectabiliteits grens. Dit laatste kan vooral optreden wanneer complicaties na colorectale chirurgie de verdere behandeling vertragen. Bij een lever-eerst strategie wordt een omgekeerde volgorde in de behandeling gehanteerd. Voordeel van de lever-eerst strategie is dat de uitzaaiingen, als belangrijkste doodsoorzaak, als eerste behandeld worden. Een ander voordeel zou kunnen zijn dat de toediening van een deel van de chemotherapie voor de operatie een kans biedt om een tumorrespons te beoordelen en daarmee het biologische gedrag van de tumor en leveruitzaaiingen te bepalen. Bovendien verschaft de lever-eerst strategie een tijdsperiode gedurende welke eventueel latent aanwezige uitzaaiingen buiten de lever een kans hebben om zichtbaar te worden.

In de studie ondergingen 18 patiënten $(81,8 \%)$ het volledige lever-eerst behandelplan voor lever en primaire tumor. Belangrijk is dat het interval tussen de operaties voor de lever en de endeldarm tumor vergelijkbaar was voor patiënten die wel en patiënten die geen com- 
plicaties na de lever operatie ontwikkelden. De overige patiënten weken af van het protocol als gevolg van het ontstaan van inoperabele uitzaaiingen buiten de lever, in de loop van hun behandeling (d.w.z. onder chemotherapie). Dit ondersteunt het mogelijke voordeel van het chirurgie-vrije interval. Patiënten kan een operatie bespaard worden in het geval van progressie van de ziekte. Vooral wanneer voor de primaire tumor een voorbehandeling met bestraling of chemotherapie geïndiceerd is en er dus een mogelijkheid voor de lever-eerst benadering ontstaat, moet in het multidisciplinaire geïndividualiseerde behandelingsplan van deze patiënten deze omgekeerde volgorde worden overwogen.

Tenslotte vormen de observaties in deze studie een argument om de behandeling van patiënten met synchrone CRCLM te beginnen met 3 cycli systemische chemotherapie, gevolgd door leverchirurgie en daarna de laatste 3 cycli chemotherapie. De operatie van dikke darm of endeldarm wordt dan uitgevoerd aan het einde van de behandel sequentie en alleen bij patiënten die daar echt nog baat bij hebben.

\section{DEEL 2 - REDUCTIE VAN DE RISICO'S VAN LEVER CHIRURGIE}

In deel 2 van dit proefschrift beschrijven we de virtuele leverresectie en lever volumetrie met 3 verschillende beeldanalyse systemen. Nauwkeurige preoperatieve risicobeoordeling ter bepaling of een patiënt een grote of uitgebreide lever resectie kan ondergaan, blijft de Heilige Graal van de lever chirurgie. Voor zover levervolume overeenkomt met leverfunctie en nauwkeurige systemen om de functie van de toekomstige restlever te voorspellen ontbreken, blijft beoordeling en voorspelling van levervolume, en met name de omvang van de toekomstige restlever essentieel. Dit is vooral van belang bij patiënten met een verminderde leverfunctie als gevolg van voorbehandeling met chemotherapie, cirrose of leververvetting. Tevens is dit belang bij patiënten die een zeer uitgebreide leverresectie moeten ondergaan.

Het lever volume kan nauwkeurig berekend worden met computertomografie (CT) en magnetische resonantie imaging (MRI) scans maar lever volumetrie software is meestal verbonden met scanner systemen en vereist een ervaren radioloog. Ook moet de voorgenomen operatie bekend zijn om het toekomstige overgebleven lever volume nauwkeurig te kunnen voorspellen en dit vereist de expertise van een lever chirurg. Wegens gebrek aan niet te dure, gebruiksvriendelijke, tijd efficiënte en gemakkelijk toegankelijke software voor lever volumetrie is het toekomstige lever volume vaak niet precies bekend bij het multidisciplinaire team dat adviseert of een leverresectie geïndiceerd en mogelijk is. De recente ontwikkeling van downloadbare open source of door de overheid gefinancierde software voor beeldanalyse en de ontwikkeling van Digital Imaging and Communications in Medicine (DICOM) standaard, hebben het mogelijk gemaakt CT of MRI lever volumetrie op een standalone of netwerk-aangesloten personal computer uit te voeren.

In hoofdstuk 4 beschrijven we het gebruik en testen we de nauwkeurigheid van de gratis 
downloadbare software ImageJ voor lever CT volumetrie bij 15 patiënten die een grote hepatectomie voor colorectale kanker uitzaaiingen ondergaan. ImageJ is ontwikkeld aan het National Institute of Health ( $\mathrm{NIH}$ ) om klinische beeld analyse te ondersteunen. In de studie blijkt dat een sterke correlatie bestaat tussen gereseceerd gewicht van de lever en preoperatief CT-gemeten levervolume, waarmee bewezen wordt dat door een chirurg nauwkeurige $\mathrm{CT}$ volumetrische analyse kan worden uitgevoerd op een personal computer. Echter, volumetrie met ImageJ is tijdrovend. Een handmatige analyse van de lever duurt 50 seconden per CT slice. Een volledige volumetrie van een hele lever duurt gemiddeld 25 tot 28 minuten. Door het verhogen van de slice dikte naar $1 \mathrm{~cm}$ werd de tijdsduur nodig voor een volumetrie verminderd zonder de nauwkeurigheid te beperken.

Onlangs is een meer geavanceerd en gebruiksvriendelijker, gratis downloadbare open source software OsiriX, beschikbaar gekomen. Het systeem is ontwikkeld door een team werkzaam aan de UCLA, Los Angeles, VS en Le Tour Ziekenhuis, Genève, Zwitserland.

In hoofdstuk 5 beschrijven we het gebruik en testen de nauwkeurigheid van OsiriX CT volumetrie bij 25 patiënten die een leverresectie ondergingen. De resultaten worden vergeleken met de lever CT volumetrie uitgevoerd door een radioloog met behulp van de standaard radiologische software (iNtuition, Terra Recon) gekoppeld aan het Maastricht UMC CT-scanner systeem. De studie laat een sterke correlatie zien tussen het chirurgisch verwijderde levergewicht en het preoperatief met OsiriX en iNtuition voorspelde levervolume. Dit bewijst dat nauwkeurige CT lever volumetrie kan worden uitgevoerd met beide systemen. De gemiddelde tijd die nodig is om het totale volume lever, tumor volume en resectie volume met OsiriX te analyseren was 19 minuten. Dit is iets sneller dan de tijd gerapporteerd voor ImageJ, wat kan worden verklaard door de halfautomatische selectie van de lever oppervlakten met OsiriX.

In de studie, ontdekten we ook dat de volumes berekend door iNtuition op onverklaarbare wijze werden veranderd wanneer het Hounsfield window niveau werd veranderd, bijvoorbeeld van buik naar lever window. Dit kan hebben geleid tot verschillen in volumetrie resultaten tussen de twee pakketten en tussen patiënten. Hierdoor rijst de vraag of iNtuition wel moet worden beschouwd als de 'goud standaard'voor klinisch gebruik. Als gevolg zocht Terra Recon, het bedrijf dat iNtuition ontwikkelt, naar een oplossing om dit ongewenste verschijnsel aan te pakken en heeft het een speciaal lever sjabloon ontwikkeld. Toekomstig onderzoek zal nodig zijn om te bepalen of dit nieuwe sjabloon nauwkeuriger is bij de beoordeling van het levervolume.

In hoofdstuk 6 vergelijken we de nauwkeurigheid in CT lever volumetrie met ImageJ, OsiriX en iNtuition bij 15 patiënten die een rechter hemihepatectomie ondergingen. Er was een sterke correlatie van de gewichten van de gereseceerde hemi-levers met de volumes geme- 
ten met radiologie software iNtuition en de volumes gemeten met ImageJ en OsiriX. Hieruit blijkt dat ImageJ en OsiriX, zelfs bij gebruik door niet-radiologen, minstens zo geschikt zijn voor CT volumetrie van de lever als het professionele software pakket iNtuition. Bovendien biedt OsiriX een belangrijk voordeel ten opzichte ImageJ omdat het een integraal beeld archief en communicatiesysteem (PACS) heeft. De CT scan en volumetrie gegevens van alle onderzochte patiënten worden automatisch opgeslagen.

\section{DEEL 3 - VERBETERING VAN DE UITKOMST VAN LEVERCHIRURGIE: \\ VERANDER DE OPERATIETECHNIEK EN VERANDER DE PERIOPERATIEVE ZORG}

Perioperatieve zorg bij patiënten die een leverresectie ondergaan is nog steeds sterk beïnvloed door dogma en traditie. Binnen het traditionele perioperatieve zorgpad varieert het mediane ziekenhuisverblijf van patiënten na leverresectie tussen 8 en 14 dagen.

Multimodale perioperatieve zorg protocollen, meestal aangeduid als fast-track of verbeterde herstel programma's, sturen patiënt verwachtingen, beïnvloeden klinische factoren die postoperatief herstel vertragen zoals pijn, darm dysfunctie en immobiliteit, en combineren een reeks van interventies om perioperatieve stress en orgaan falen te verminderen. Dergelijke programma's stellen goed geïnformeerde patiënten in staat om actief deel te nemen aan het herstel proces. Ze versnellen het postoperatieve herstel en verminderen daarmee de lengte van het ziekenhuisverblijf.

In hoofdstuk 7 beschrijven we de implementatie studie van het evidence-based multimodale Enhanced Recovery After Surgery (ERAS) lever-programma, nieuw ontwikkeld door de levercentra van de internationale ERAS-groep in Maastricht, Edinburgh en Tromsø. Het verbeterde herstel protocol werd prospectief onderzocht in een opeenvolgende reeks van 61 patiënten die een leverresectie in het Maastricht Universitair Medisch Centrum of in de Royal Infirmary of Edinburgh, Verenigd Koninkrijk ondergingen. Resultaten werden vergeleken met die van een opeenvolgende reeks van 100 patiënten die leverresectie ondergingen in een van beide klinieken voor de implementatie van het ERAS lever-programma, toen een meer traditioneel zorgpad nog in gebruik was.

De studie toonde aan dat het gebruik van het versneld herstelprogramma postoperatief herstel versnelt en leidt tot een significant korter ziekenhuisverblijf. De meeste patiënten die volgens het ERAS-protocol behandeld waren, waren in staat om binnen 4 uur na leverresectie te drinken, normaal voedsel te eten op de dag na de operatie en werden op de vijfde dag na de operatie uit het ziekenhuis ontslagen. Bovendien is het programma veilig gebleken, omdat er geen significante verschillen waren in het aantal heropnames, complicaties en mortaliteit tussen ERAS en controlegroepen.

Een andere trend is de groeiende voorkeur van chirurgen en patiënten voor de laparosco- 
pische leverresectie t.o.v. open resectie. Laparoscopische leverresectie kan veel voordelen hebben ten opzichte van open leverresectie. Diverse publicaties in de afgelopen 15 jaar presenteren gegevens van meer dan $\mathbf{4 0 0 0}$ patiënten en laten consequent zien dat deze patiënten minder last van pijn, een sneller herstel en een korter ziekenhuisverblijf hebben. Echter, al deze uitkomsten zijn waarschijnlijk onderhevig aan bias omdat ze nooit onderzocht zijn in een gerandomiseerd gecontroleerde studie waarin open en laparoscopische leverresectie worden vergeleken.

Het is pas sinds kort dat de chirurgische gemeenschap het idee heeft aanvaard dat beide strategieën synergetisch kunnen zijn. De laparoscopische benadering is een manier om de chirurgische ingreep voor de patiënt te beperken en kan worden gezien als een hedendaags kernelement van het ERAS programma.

In hoofdstuk 8 beschrijven we een retrospectieve, multicenter studie naar het effect van een ERAS programma bij patiënten die een laparoscopische leverresectie ondergingen. 26 patienten werden behandeld door laparoscopische leverresectie gedurende een periode van 5 jaar. Uitkomsten van dertien patiënten die laparoscopische leverresecties binnen het ERAS programma in het Maastricht UMC ondergingen werden vergeleken met dertien patiënten die een laparoscopische leverresectie in een traditioneel perioperatief zorgpad in het Maastricht UMC ( $n=3)$, AMC Amsterdam ( $n=4)$, UMC Utrecht $(n=2)$ en UMC Groningen $(n=4)$ ondergingen. Hoewel het verschil klein is, suggereert de studie dat een multimodaal verbeterd herstelprogramma in laparoscopische lever chirurgie haalbaar en veilig is en kan leiden tot versneld functioneel herstel en een verkorting van de duur van het ziekenhuisverblijf.

Voorlopig blijft het onduidelijk welk deel van de kortere opnameduur voortvloeit uit de invoering van een formeel zorgpad en welk deel voortvloeit uit de ERAS kernelementen van dat zorgpad. Met behulp van adequaat gepowerde en goed ontworpen multicenter trials moet de waarde van de verschillende kernelementen van het ERAS versneld herstel programma één voor één worden vastgesteld.

Daarnaast wordt meer en meer erkend dat de lengte van ziekenhuisverblijf, de meest gebruikte uitkomstmaat in studies over de waarde van de laparoscopische techniek of versneld herstel programma's, niet alleen afhankelijk is van het herstel, maar ook sterk beïnvloed wordt door de verwachtingen van de patiënt en de ontslagplanning van het ziekenhuispersoneel. Tijdsduur tot functioneel herstel in plaats van lengte van het ziekenhuisverblijf kan een betere uitkomstmaat zijn voor toekomstig onderzoek. Een patiënt is volledig functioneel hersteld of medisch klaar voor ontslag als aan de volgende vijf criteria wordt voldaan: Adequate pijnbestrijding met orale pijnstillers, gemobiliseerd op preoperatief niveau, tolerantie van vast voedsel, normaal of te verlagen bilirubine en INR van ten minste $80 \%$ van de normale waarde, geen intraveneuze vloeistoffen. 
In hoofdstuk 9 beschrijven we de gerandomiseerd gecontroleerde ORANGE trial (Optimized Recovery with Accelerated Nutrition and Gastrointestinal Enhancement). In dit onderzoek bekijken we door twee elementen van het ERAS-protocol aan te passen of postoperatieve darm stimulatie met magnesium hydroxide en / of orale drinkvoeding (ONS) effectief is in het bevorderen van de terugkeer van gastro-intestinale functies en het algehele postoperatief herstel na lever resectie.

68 patiënten die in de Royal Infirmary of Edinburgh, Verenigd Koninkrijk of het Maastricht Universitair Medisch Centrum een leverresectie ondergingen werden gerandomiseerd. De studie toonde aan dat de meeste patiënten op de dag van de operatie weer water dronken en de dag na de operatie weer begonnen met eten van vast voedsel. De patiënten waren op de derde dag volledig gemobiliseerd en voldeden op de vierde dag na de operatie aan de vooraf gedefinieerde ontslagcriteria. Het gebruik van laxeermiddelen zorgde voor een significante eerdere stoelgang. Daarentegen was in de postoperatieve fase het aantal ingenomen calorieën van patiënten in de groep met drinkvoeding niet anders dan die van patienten die geen drinkvoeding ontvingen. Dit suggereert dat het gebruik van drinkvoeding in de vroege postoperatieve fase normale voedselinname onderdrukt tot het punt waarop er geen netto voordeel is. Het mediane ziekenhuisverblijf was 6 dagen. Dit is aanzienlijk korter dan de 8-14 dagen gemeld in andere centra met traditionele perioperatieve zorg. Functioneel herstel werd mediaan op 4 dagen na de operatie bereikt, gemiddeld 2 dagen voor ontslag uit het ziekenhuis.

In hoofdstuk 10 presenteren we de resultaten van een internationale enquête onder 161 hepatopancreatobiliaire (HPB) centra wereldwijd. Het toont aan dat het gebruik van verbeterde herstelprogramma's in de lever chirurgie beperkt is en rechtvaardigt meer aandacht voor deze programma's in de HPB gemeenschap. Het onderzoek toont ook aan dat de meerderheid van de HPB centra laparoscopische leveroperaties uitvoert, hoewel het aantal laparoscopische resecties in het merendeel van de centra laag is. Tevens vindt meer dan de helft van de HPB centra het noodzakelijk dat in een gerandomiseerd gecontroleerde studie de resultaten van open en laparoscopische lever chirurgie worden vergeleken voordat besloten wordt of verdere invoering van de laparoscopische lever chirurgie nodig is.

In hoofdstuk 11 presenteren we de studieprotocollen van 2 gerandomiseerd gecontroleerde studies die internationaal multicentrisch actief includeren. De studies vergelijken open en laparoscopische linker laterale sectionectomie (ORANGE II) of hemihepatectomie (ORANGE II Plus) binnen een ERAS programma.

Tijdsduur tot functioneel herstel is het primaire eindpunt van zowel de ORANGE II als ORANGE II Plus trial. Secundaire eindpunten zijn postoperatieve duur van ziekenhuisverblijf, percentage heropnamen, een samengesteld eindpunt van leverchirurgie specifieke com- 
plicaties, kwaliteit van leven, lichaamsbeeld en cosmetisch resultaat, ziekenhuis en maatschappelijke kosten gedurende 1 jaar, ontstaan van littekenbreuken op lange termijn en overlevingsduur bij oncologische patiënten. In beide studies wordt aangenomen dat de periode tot functioneel herstel na laparoscopische resectie kan worden verminderd met twee dagen ten opzichte van open resectie. Steekproefomvang voor de ORANGE II studie is berekend op 55 patiënten in elke gerandomiseerde groep. Steekproefomvang voor de ORANGE II Plus studie is berekend op 125 patiënten in elke gerandomiseerde groep.

De vraag blijft of een gerandomiseerd gecontroleerde studie, met al zijn moeilijkheden, noodzakelijk is om te bewijzen dat laparoscopie moet worden aanvaard als voorkeurs techniek van een lever resectie. Chirurgen en patiënten in ervaren centra zullen aarzelen om patiënten te randomiseren omdat er een gevoelsmatige voorkeur is voor de laparoscopische resectie. Ook onervaren chirurgen kunnen aarzelen omdat ze zich de techniek van de laparoscopische leverresectie eigen willen maken en de aantallen in hun leercurve nodig hebben. Er zijn zelfs ervaren chirurgen die beweren dat het randomiseren van patiënten onethisch is, omdat naar hun mening, er al genoeg uitkomst gegevens gepubliceerd zijn.

Echter, in de meeste gerapporteerde onderzoeken of systematische reviews die gericht zijn op sneller herstel, minder pijn of een vermindering van opnameduur na laparoscopische leverresectie, waren type resectie en / of perioperatieve zorg niet gestandaardiseerd. Meestal is het niet duidelijk welke definities werden gebruikt voor specifieke uitkomstmaten en hoe ze werden geregistreerd. Tevens wordt het steeds duidelijker dat de ziekenhuisverblijfsduur een onbetrouwbare uitkomstmaat is omdat het te afhankelijk is van patiënt verwachtingen, planning, cultuur, ziekenhuis logistiek en vergoedingen.

In de ORANGE II studies voorziet het ERAS versneld herstel protocol in de gewenste perioperatieve standaardisatie. De primaire uitkomst parameter functioneel herstel/medisch klaar voor ontslag is goed meetbaar en gebaseerd op harde klinische herstel criteria. Het gebruik van een ervaren gast chirurg in centra met een beperkte ervaring in laparoscopische leverchirurgie moet de invloed van de leercurve op de uitkomst elimineren. De economische evaluatie zal een kosten-batenanalyse vanuit ziekenhuis en Nederlandse maatschappelijk perspectief bevatten en gegevens verstrekken over de kosten per quality adjusted life year (QALY).

De opzet, waarin een gerandomiseerd gecontroleerde studie en een prospectieve registratie gecombineerd worden, zal de power, de externe validiteit en generaliseerbaarheid van studieresultaten versterken. 


\section{ALGEMENE DISCUSSIE ENTOEKOMST PERSPECTIEH.}

In de afgelopen eeuw is het aantal wereldwijd uitgevoerde leverresecties enorm toegenomen. Deze toename is mogelijk geworden door het continue streven naar verbetering van de patiëntenzorg. Recidief resecties, gepland gefaseerde leverresecties,

leverresecties na eenzijdige embolisatie van de poortader en de lever eerst benadering hebben allemaal het licht gezien. Combinaties met inductie van chemotherapie, radiofrequente ablatie, trans arteriële chemo embolisatie, in situ perfusie en Gamma Knife of stereotactische radiotherapie zijn geïntroduceerd. Al deze ontwikkelingen hebben het chirurgisch instrumentarium om primaire of secundaire levertumoren te bestrijden, vergroot.

Het probleem met de meeste nieuwe chirurgische procedures is dat ze op een tamelijk ongecontroleerd wijze zijn geïntroduceerd. Geen van de bovengemelde procedures is onderworpen aan de juiste testen in gerandomiseerd gecontroleerde studies, waardoor patiënten mogelijk kunnen zijn blootgesteld aan onnodige risico's. Dit moet te veranderen. Gerandomiseerd gecontroleeerde onderzoeken zijn echter moeilijk uit te voeren in de chirurgie. Good Clinical Practice regels en procedures zijn omslachtig en tijdrovend en de frequentie van sommige van de belangrijkste resultaten in de lever chirurgie, zoals gal lekkage, leverfalen en sterfte, is te laag om een onderzoek uitvoerbaar te maken in termen van voldoende patiëntdeelname of tijdsduur van het onderzoek. Om dit probleem op te lossen hebben we een samengesteld, lever specifiek eindpunt ontwikkeld, dat bestaat uit een combinatie van klinisch relevante lever specifieke individuele eindpunten, met elk een vrij lage frequentie na leverresecties. In gecombineerde vorm neemt de frequentie van het lever specifieke gecombineerde eindpunt toe, hetgeen de haalbaarheid van het uitvoeren van een gerandomiseerd gecontroleerd onderzoek door een aanzienlijk kleinere steekproefomvang verhoogt. Bovendien kan consequent gebruik van dit leverchirurgie specifieke gecombineerde eindpunt de vergelijkbaarheid van trials en de kwaliteit van toekomstige meta-analyses verbeteren.

De wijze waarop patiënten worden voorbereid op vaak een reeks ingrepen alsmede de organisatie van de zorg na deze ingrepen zijn van grote invloed op de uitkomst.

In het Maastricht Universitair Medisch Centrum hebben we uitgebreide ervaring opgedaan met de implementatie van best practice perioperatieve zorg voor patiënten: het zogenaamde Enhanced Recovery After Surgery of fast-track programma. Dit perioperatieve zorg programma leidt tot een sneller herstel dankzij een voorlichting, optimale pijnbestrijding, vroeg hervatten van voeding, minder slangen en drains, en actieve mobilisatie na de operatie. Als gevolg hiervan verlaten patiënten sneller en in beter conditie het ziekenhuis. Dit blijkt kosten te verlagen en maakt tevens een vroegere start van aanvullende chemotherapie bij patiënten die kanker chirurgie ondergingen, mogelijk. 
Recent hebben we aangetoond dat het zelfs vandaag de dag nog meer dan een decennium duurt om de opgedane kennis wereldwijd te implementeren in de algemeen chirurgische praktijk. Samenwerkingsinitiatieven en dataregistratie van de Nederlandse Lever Werkgroep, het Nederlands Instituut voor Clinical Auditing en de internationale Enhanced Recovery after Surgery Groep kunnen helpen om de implementatie van de opgedane kennis te versnellen. Het gebruik van wereldwijd geaccepteerde welomschreven resultaten- en classificatiesystemen is een voorwaarde om alle onderzoeken en controles onvoorwaardelijk met elkaar te kunnen vergelijken. De Accordion ${ }^{\circledast}$, gemodificeerde Clavien-Dindo-Strasberg, classificatiesysteem van postoperatieve chirurgische complicaties is het best omschreven classificatiesysteem maar houdt geen rekening met intra-operatieve complicaties. Het Satava-classificatiesysteem voor intra-operatieve complicaties zou het meest geschikte systeem kunnen zijn om dat tekort aan te vullen.

Tot voor kort, maar ook vandaag de dag nog, werd een leveroperatie niet aangeboden aan alle daarvoor in aanmerking komende patiënten. Dit was niet gebaseerd op objectieve criteria en het is zeer waarschijnlijk dat veel patiënten een operatie is onthouden die hen had kunnen genezen. Onwetendheid over oncologische uitkomst van niet-gespecialiseerde teams en het ontbreken van criteria om de lichamelijke veerkracht van patiënten tot chirurgie en de complicaties ervan te meten, zijn hiervoor verantwoordelijk. Het is duidelijk dat elke patiënt met colorectale kanker uitzaaiingen, maar waarschijnlijk ook elke patiënt met een levertumor, moet worden besproken in een gespecialiseerd lever- en darm-kanker multidisciplinair teamoverleg. Alleen op deze manier zullen patiënten toegang hebben tot optimale deskundigheid en behandeling. Daarom moet de organisatie van zorg niet alleen gericht zijn op het voorkomen van complicaties, maar ook op het redden van patiënten van vaak levensbedreigende complicaties, mochten deze optreden. Een gespecialiseerd multidisciplinair hepatobiliair team dat deze patiënten op een 24/7 basis in de gaten houdt, onderstreept het belang van centralisatie in hoog-volume ziekenhuizen. Bovendien moet een beoordeling van geschiktheid om de stress van mogelijke complicaties te weerstaan, gebaseerd op objectieve criteria, worden ontwikkeld. Evaluatie van de patiënt met behulp van preoperatieve cardiopulmonale inspanningstesten en CT-gebaseerde volumetrische meting van spiermassa om de voedingstoestand te beoordelen kunnen helpen om patiënten te selecteren op hoger risico bij een leveroperatie.

Pas recent zijn geïndividualiseerde patiëntenzorg en de veiligheid van de patiënt in toenemende mate op de agenda gezet. Het gebruik van gestructureerde geïnformeerde toestemming, checklists, aan- en afmeld procedures en stop-momenten in chirurgische procedures hebben sterk bijgedragen aan de veiligheid van patiënten. Dit zal ongetwijfeld tot uiting komen in patiënt uitkomst evaluaties, zoals voorgesteld door patiënt organisaties. Geïndividualiseerde patiëntenzorg, hoewel meer en meer mogelijk, kan in strijd zijn met het maatschappelijke streven naar meer kosteneffectieve behandelingen. Natuurlijk moet het 
behandelvoorstel per patiënt worden afgestemd en geïndividualiseerd zijn. Maar de aanvaarding van een behandel voorstel door de patiënt is vervolgens een gezamenlijke beslissing van de patiënt en de behandelaar. Het is duidelijk dat patiënten vaak sterke voorkeuren hebben en dat hun voorkeuren niet altijd samenvallen met die van hun artsen, de samenleving en de richtlijnen. In deze context is het belangrijk te beseffen dat medisch specialisten, de samenleving en patiënten verschillend oordelen over het voordeel van een behandeling. In het verkennen van de grenzen van de resectie leverchirurgie benadrukken veel leden van een multidisciplinair team wat er mis kan gaan, terwijl de meeste patiënten bereid zijn om een berekend risico te nemen op basis van wat goed kan gaan. 
LIST OF

PUBLICATIONS 
Preoperative Characteristics of Patients with Presumed Pancreatic Cancer but Ultimately Benign Disease:

A Multicenter Series of 344 Pancreatoduodenectomies. Gerritsen A, Molenaar IQ, Bollen TL, Nio CY, Dijkgraaf MG, van Santvoort HC, Offerhaus GJ, Brosens LA, Biermann $K$, Sieders $E$, de Jong $K P$, van Dam RM, van der Harst $E$, van Goor $\mathrm{H}$, van Ramshorst $\mathrm{B}$, Bonsing $\mathrm{BA}$, de Hingh $\mathrm{IH}$, Gerhards MF, van Eijck CH, Gouma DJ, Borel Rinkes IH, Busch OR, Besselink MG; Dutch Pancreatic Cancer Group. Ann Surg Oncol. 2014 May 29.

A combined measure of procedural volume and outcome to assess hospital quality of colorectal cancer surgery, a secondary analysis of clinical audit data.

Kolfschoten NE, Marang-van de Mheen PJ, Wouters MW, Eddes EH, Tollenaar RA, Stijnen T, Kievit J; Dutch Surgical Colorectal Audit Group.

PLoS One. 2014 Feb 18;9(2):e88737.

doi: 10.1371/journal.pone.0088737. eCollection 2014.

Obstructive putty-like cast of the biliary tree.

Clermonts SH, van Dam RM.

Hepatobiliary Surg Nutr. 2014 Feb;3(1):47-9.

doi: 10.3978/j.issn.2304-3881.2014.01.01

Development of a composite endpoint for randomized controlled trials in pancreaticoduodenectomy.

Coolsen MM, Clermonts SH, van Dam RM, Winkens B, Malagó M, Fusai GK, Dejong CH, Olde Damink SW.

World J Surg. 2014 Jun;38(6):1468-75.

doi: 10.1007/s00268-013-2421-y.

Is current perioperative practice in hepatic surgery based on enhanced recovery after surgery (ERAS) principles? Wong-Lun-Hing EM, van Dam RM, Heijnen LA, Busch OR, Terkivatan $T$, van Hillegersberg R, Slooter GD, Klaase J, de Wilt JH, Bosscha K, Neumann UP, Topal B, Aldrighetti LA, Dejong $\mathrm{CH}$.

World J Surg. 2014 May;38(5):1127-40.

doi: 10.1007/s00268-013-2398-6.

The role of routine fine-needle aspiration in the diagnosis of infected necrotizing pancreatitis.

van Baal MC, Bollen TL, Bakker OJ, van Goor $\mathrm{H}$, Boermeester MA, Dejong $\mathrm{CH}$, Gooszen HG, van der Harst E, van Eijck CH, van Santvoort HC, Besselink MG; Dutch Pancreatitis Study Group.

Surgery. 2014 Mar;155(3):442-8. doi: 10.1016/j.surg.2013.10.001.

Epub 2013 Oct 12.

Outcomes of extended versus limited indications for patients undergoing a liver resection for colorectal cancer liver metastases.

van Dam RM, Lodewick TM, van den Broek MA, de Jong MC, Greve JW, Jansen RL, Bemelmans MH, Neumann UP, Olde Damink SW, Dejong CH.

HPB (Oxford). 2014 Jun;16(6):550-9.

doi: 10.1111/hpb.12181. Epub 2013 Nov 7

Postoperative pain control using continuous i.m. bupivacaine infusion plus patient-controlled analgesia compared with epidural analgesia after major hepatectomy. Wong-Lun-Hing EM, van Dam RM, Welsh FK, Wells JK, John TG, Cresswell AB, Dejong CH, Rees M.

HPB (Oxford). 2014 Jul;16(7):601-9.

doi: $10.1111 / \mathrm{hpb} .12183$.

Epub 2013 Oct 23.

Influence of preoperative chemotherapy on ct volumetric liver regeneration following right hemihepatectomy. Dello SA, Kele PG, Porte RJ, van Dam RM, Klaase JM, Verhoef C, van Gulik T, Molenaar Q, Bosscha K, van der Jagt EJ, Dejong $\mathrm{CH}$, de Boer MT.

World J Surg. 2014 Feb;38(2):497-504.

doi: 10.1007/s00268-013-2278-0.

Plasma intestinal fatty acid-binding protein levels correlate with morphologic epithelial intestinal damage in a human translational ischemia-reperfusion model.

Schellekens DH, Grootjans J, Dello SA, van Bijnen AA, van Dam RM, Dejong CH, Derikx JP, Buurman WA.

J Clin Gastroenterol. 2014 Mar;48(3):253-60.

doi: 10.1097/MCG.0b013e3182a87e3e

Combined vascular and biliary fluorescence imaging in laparoscopic cholecystectomy.

Schols RM, Bouvy ND, van Dam RM, Masclee AA, Dejong $\mathrm{CH}$, Stassen LP.

Surg Endosc. 2013 Dec;27(12):4511-7.

doi: 10.1007/s00464-013-3100-7.

Epub 2013 Jul 23.

Evaluating the validity of quality indicators for colorectal cancer care.

Gooiker GA, Kolfschoten NE, Bastiaannet E, van de Velde $\mathrm{CJ}$, Eddes EH, van der Harst E, Wiggers T, Rosendaal FR, 
Tollenaar RA, Wouters MW; Dutch Surgical Colorectal Audit group.

J Surg Oncol. 2013 Dec;108(7):465-71.

doi: $10.1002 /$ jso. 23420 .

Epub 2013 Sep 20.

Identification and validation of risk factors for postoperative infectious complications following hepatectomy.

Pessaux P, van den Broek MA, Wu T, Olde Damink SW, Piardi T, Dejong $\mathrm{CH}$, Ntourakis D, van Dam RM.

J Gastrointest Surg. 2013 Nov; 17(11):1907-16.

doi: 10.1007/s11605-013-2226-1.

Epub 2013 May 10.

Erratum in: J Gastrointest Surg. 2013 Nov;17(11):2032.

Systematic review and meta-analysis of enhanced recovery after pancreatic surgery with particular emphasis on pancreaticoduodenectomies.

Coolsen MM, van Dam RM, van der Wilt AA, Slim K, Lassen K, Dejong $\mathrm{CH}$.

World J Surg. 2013 Aug;37(8):1909-18.

doi: 10.1007/s00268-013-2044-3.

The Dutch surgical colorectal audit.

Van Leersum NJ, Snijders HS, Henneman D, Kolfschoten NE, Gooiker GA, ten Berge MG, Eddes EH, Wouters MW, Tollenaar RA; Dutch Surgical Colorectal Cancer Audit Group, Bemelman WA, van Dam RM, Elferink MA, Karsten $T M$, van Krieken JH, Lemmens VE, Rutten HJ, Manusama $E R$, van de Velde CJ, Meijerink WJ, Wiggers T, van der Harst E, Dekker JW, Boerma D.

Eur J Surg Oncol. 2013 Oct;39(10):1063-70.

doi: 10.1016/j.ejso.2013.05.008.

Epub 2013 Jul 18.

To drain or not to drain: a cumulative meta-analysis of the use of routine abdominal drains after pancreatic resection.

Van der Wilt AA, Coolsen MM, de Hingh IH, van der Wilt GJ, Groenewoud H, Dejong CH, van Dam RM

PB (Oxford). 2013 May;15(5):337-44.

Epub 2012 Nov 30. 995;8(3):201-6

A systematic review of outcomes in patients undergoing liver surgery in an enhanced recovery after surgery pathways.

Coolsen MM, Wong-Lun-Hing EM, van Dam RM, van der Wilt AA, Slim K, Lassen K, Dejong CH.
HPB (Oxford). 2013 Apr;15(4):245-51. doi: 10.1111/j.1477-2574.2012.00572.x. Epub 2012 Sep 28.

Advanced intraoperative imaging methods for laparoscopic anatomy navigation: an overview.

Schols RM, Bouvy ND, van Dam RM, Stassen LP.

Surg Endosc. 2013 Jun;27(6):1851-9.

doi: 10.1007/s00464-012-2701-x.

Epub 2012 Dec 14. Review

Nonelective colon cancer resections in elderly patients: results from the dutch surgical colorectal audit.

Kolfschoten NE, Wouters MW, Gooiker GA, Eddes EH, Kievit J, Tollenaar RA, Marang-van de Mheen PJ;

Dutch Surgical Colorectal Audit group.

Dig Surg. 2012;29(5):412-9.

doi: $10.1159 / 000345614$.

Epub 2012 Dec 13.

A survey in the hepatopancreatobiliary community on ways to enhance patient recovery.

Wong-Lun-Hing EM, Lodewick TM, Stoot JH, Bemelmans $\mathrm{MH}$, Olde Damink SW, Dejong CH, van Dam RM.

HPB (Oxford). 2012 Dec;14(12):818-27.

doi: 10.1111/j.1477-2574.2012.00546.x.

Epub 2012 Aug 26.

Erratum to: Influence of Comorbidity and Age on 1-, 2-, and 3-Month Postoperative Mortality Rates in Gastrointestinal Cancer Patients.

van Gestel YR, Lemmens VE, de Hingh IH, Steevens J, Rutten HJ, Nieuwenhuijzen GA, van Dam RM, Siersema PD. Ann Surg Oncol. 2012 Nov 7. [Epub ahead of print] No abstract available.

Clinical outcome in relation to timing of surgery in chronic pancreatitis: a nomogram to predict pain relief.

Ahmed Ali U, Nieuwenhuijs VB, van Eijck $\mathrm{CH}$, Gooszen HG, van Dam RM, Busch OR, Dijkgraaf MG, Mauritz FA, Jens S, Mast J, van Goor H, Boermeester MA; Dutch Pancreatitis Study Group.

Arch Surg. 2012 Oct;147(10):925-32.

doi: 10.1001/archsurg.2012.1094.

Fluorescence cholangiography during laparoscopic cholecystectomy: a feasibility study on early biliary tract delineation. 
Schols RM, Bouvy ND, Masclee AA, van Dam RM, Dejong $\mathrm{CH}$, Stassen LP.

Surg Endosc. 2013 May;27(5):1530-6.

doi: 10.1007/s00464-012-2635-3.

Epub 2012 Oct 18

European experience of laparoscopic major hepatectomy. Tzanis D, Shivathirthan N, Laurent A, Abu Hilal M, Soubrane O, Kazaryan AM, Ettore GM, Van Dam RM, Lainas $P$, Tranchart H, Edwin B, Belli G, Campos RR, Pearce N, Gayet B, Dagher I. J Hepatobiliary Pancreat Sci. 2013 Feb;20(2):120-4.

doi: 10.1007/s00534-012-0554-2.

Sarcopenia negatively affects preoperative total functional liver volume in patients undergoing liver resection. Dello SA, Lodewick TM, van Dam RM, Reisinger KW, van den Broek MA, von Meyenfeldt MF, Bemelmans MH, Olde Damink SW, Dejong $\mathrm{CH}$.

HPB (Oxford). 2013 Mar;15(3):165-9.

doi: 10.1111/j.1477-2574.2012.00517.x.

Epub 2012 Jun 21.

Influence of comorbidity and age on 1-, 2-, and 3-month postoperative mortality rates in gastrointestinal cancer patients.

van Gestel YR, Lemmens VE, de Hingh IH, Steevens J, Rutten HJ, Nieuwenhuijzen GA, van Dam RM, Siersema PD.

Ann Surg Oncol. 2013 Feb;20(2):371-80. doi: 10.1245/ s10434-012-2663-1.

Epub 2012 Sep 18.

Fibrin sealant for prevention of resection surface-related complications after liver resection: a randomized controlled trial.

de Boer MT, Klaase JM, Verhoef C, van Dam RM, van Gulik TM, Molenaar IQ, Bosscha K, Dejong CH, Van der Jagt EJ, Porte RJ; FRESCO Trial Group.

Ann Surg. 2012 Aug;256(2):229-34.

doi: 10.1097/SLA.0b013e3182602819.

Real-time in vivo imaging of early mucosal changes during ischemia-reperfusion in human jejunum.

Grootjans J, Hameeteman W, Masclee AA, van Dam RM, Buurman WA, Dejong $\mathrm{CH}$.

PLoS One. 2012;7(6):e39638.

doi: 10.1371 /journal.pone.0039638.

Epub 2012 Jun 22.
Open versus laparoscopic left lateral hepatic sectionectomy within an enhanced recovery ERAS ${ }^{\circledR}$ programme (ORANGE II-trial): study protocol for a randomised controlled trial.

van Dam RM, Wong-Lun-Hing EM, van Breukelen GJ, Stoot JH, van der Vorst JR, Bemelmans MH, Olde Damink SW, Lassen K, Dejong CH; ORANGE II Study Group.

Trials. 2012 May 6;13:54.

doi: 10.1186/1745-6215-13-54.

Combining process indicators to evaluate quality of care for surgical patients with colorectal cancer: are scores consistent with short-term outcome?

Kolfschoten NE, Gooiker GA, Bastiaannet E, van Leersum $\mathrm{NJ}$, van de Velde $\mathrm{CJ}$, Eddes EH, Marang-van de Mheen PJ, Kievit J, van der Harst E, Wiggers T, Wouters MW, Tollenaar RA; Dutch Surgical Colorectal Audit group.

BMJ Qual Saf. 2012 Jun;21(6):481-9.

doi: 10.1136/bmjqs-2011-000439.

Epub 2012 Apr 4.

Laparoscopic liver resection in the Netherlands: how far are we?

Stoot JH, Wong-Lun-Hing EM, Limantoro I, Visschers R, Busch OR, Van Hillegersberg R, De Jong KM, Rijken AM, Kazemier G, Olde Damink SW, Lodewick TM, Bemelmans MH, van Dam RM, Dejong CH; Dutch Liver Collaborative Group.

Dig Surg. 2012;29(1):70-8.

doi: 10.1159/000335739.

Epub 2012 Mar 15. Review.

The introduction of a laparoscopic liver surgery programme: a cost analysis of initial experience in a university hospital.

Stoot JH, van Dam RM, Coelen RJ, Winkens B, Olde Damink SW, Bemelmans MH, Dejong $\mathrm{CH}$.

Scand J Surg. 2012;101(1):32-7.

Total intermittent Pringle maneuver during liver resection can induce intestinal epithelial cell damage and endotoxemia.

Dello SA, Reisinger KW, van Dam RM, Bemelmans $\mathrm{MH}$, van Kuppevelt $\mathrm{TH}$, van den Broek MA, Olde Damink SW, Poeze M, Buurman WA, Dejong $\mathrm{CH}$.

PLoS One. 2012;7(1):e30539.

doi: 10.1371 /journal.pone.0030539.

Epub 2012 Jan 24. 
[Better pain management in chronic pancreatitis through early surgery?].

Ahmed Ali U, Bruno MJ, Issa Y, Gooszen HG, Fockens P, Boermeester MA; Pancreatitis Werkgroep Nederland. Ned Tijdschr Geneeskd. 2012;156(5):A4469.

Wait-and-see policy for clinical complete responders after chemoradiation for rectal cancer.

Maas M, Beets-Tan RG, Lambregts DM, Lammering G, Nelemans PJ, Engelen SM, van Dam RM, Jansen RL, Sosef M, Leijtens JW, Hulsewé KW, Buijsen J, Beets GL.

J Clin Oncol. 2011 Dec 10;29(35):4633-40.

doi: 10.1200/JCO.2011.37.7176.

Epub 2011 Nov 7.

Which fast track elements predict early recovery after colon cancer surgery?

Vlug MS, Bartels SA, Wind J, Ubbink DT, Hollmann MW, Bemelman WA; Collaborative LAFA Study Group.

Colorectal Dis. 2012 Aug;14(8):1001-8.

doi: 10.1111/j.1463-1318.2011.02854.x.

Variation in case-mix between hospitals treating colorectal cancer patients in the Netherlands.

Kolfschoten NE, Marang van de Mheen PJ, Gooiker GA, Eddes EH, Kievit J, Tollenaar RA, Wouters MW;

Dutch Surgical Colorectal Audit group.

Eur J Surg Oncol. 2011 Nov;37(11):956-63.

doi: 10.1016/j.ejso.2011.08.137.

Epub 2011 Sep 22.

The liver-first approach for synchronous colorectal liver metastasis: a 5-year single-centre experience.

de Jong MC, van Dam RM, Maas M, Bemelmans MH, Olde Damink SW, Beets GL, Dejong CH.

HPB (Oxford). 2011 Oct;13(10):745-52.

doi: 10.1111/j.1477-2574.2011.00372.x.

Epub 2011 Sep 5.

A conservative and minimally invasive approach to necrotizing pancreatitis improves outcome.

van Santvoort HC, Bakker OJ, Bollen TL, Besselink MG, Ahmed Ali U, Schrijver AM, Boermeester MA, van Goor H, Dejong $\mathrm{CH}$, van Eijck $\mathrm{CH}$, van Ramshorst $\mathrm{B}$, Schaapherder $A F$, van der Harst E, Hofker S, Nieuwenhuijs VB, Brink MA, Kruyt PM, Manusama ER, van der Schelling GP, Karsten $T$, Hesselink EJ, van Laarhoven CJ, Rosman C, Bosscha K, de Wit RJ, Houdijk AP, Cuesta MA, Wahab PJ, Gooszen HG;
Dutch Pancreatitis Study Group.

Gastroenterology. 2011 Oct;141(4):1254-63.

doi: 10.1053/j.gastro.2011.06.073.

Epub 2011 Jul 8.

Laparoscopy in combination with fast track multimodal management is the best perioperative strategy in patients undergoing colonic surgery: a randomized clinical trial (LAFA-study).

Vlug MS, Wind J, Hollmann MW, Ubbink DT, Cense HA, Engel AF, Gerhards MF, van Wagensveld $B A$, van der Zaag ES, van Geloven AA, Sprangers MA, Cuesta MA, Bemelman WA; LAFA study group.

Ann Surg. 2011 Dec;254(6):868-75.

doi: $10.1097 /$ SLA.0b013e31821fd1ce.

Development of a composite endpoint for randomized controlled trials in liver surgery.

van den Broek MA, van Dam RM, van Breukelen GJ, Bemelmans $\mathrm{MH}$, Oussoultzoglou E, Pessaux P, Dejong $\mathrm{CH}$, Freemantle N, Olde Damink SW.

Br J Surg. 2011 Aug;98(8):1138-45.

doi: 10.1002/bjs.7503.

Epub 2011 May 6.

Rapid lamina propria retraction and zipper-like constriction of the epithelium preserves the epithelial lining in human small intestine exposed to ischaemia-reperfusion.

Grootjans J, Thuijls G, Derikx JP, van Dam RM, Dejong CH, Buurman WA.

J Pathol. 2011 Jul;224(3):411-9.

doi: 10.1002/path.2882.

Epub 2011 May 5.

Gut and liver handling of interleukin- 6 during liver resection in man.

Dello SA, Bloemen JG, van de Poll MC, van Dam RM, Stoot $\mathrm{JH}$, van den Broek MA, Buurman WA, Bemelmans MH, Olde Damink SW, Dejong $\mathrm{CH}$. HPB (Oxford). 2011 May;13(5):324-31. doi: 10.1111/j.1477-2574.2010.00289.x. Epub 2011 Mar 7.

Prospective volumetric assessment of the liver on a personal computer by nonradiologists prior to partial hepatectomy.

Dello SA, Stoot JH, van Stiphout RS, Bloemen JG, 
Wigmore SJ, Dejong CH, van Dam RM. World J Surg. 2011 Feb;35(2):386-92. doi: 10.1007/s00268-010-0877-6.

Evaluation of management of desmoid tumours associated with familial adenomatous polyposis in Dutch patients.

Nieuwenhuis MH, Mathus-Vliegen EM, Baeten CG, Nagengast FM, van der Bijl J, van Dalsen AD, Kleibeuker JH, Dekker E, Langers AM, Vecht J, Peters FT, van Dam R, van Gemert WG, Stuifbergen WN, Schouten WR, Gelderblom H, Vasen HF.

Br J Cancer. 2011 Jan 4;104(1):37-42.

doi: 10.1038/sj.bjc.6605997.

Epub 2010 Nov 9.

Level of activation of the unfolded protein response correlates with Paneth cell apoptosis in human small intestine exposed to ischemia/reperfusion.

Grootjans J, Hodin CM, de Haan JJ, Derikx JP, Rouschop KM, Verheyen FK, van Dam RM, Dejong CH, Buurman WA, Lenaerts $\mathrm{K}$.

Gastroenterology. 2011 Feb;140(2):529-539.e3.

doi: 10.1053/j.gastro.2010.10.040.

Epub 2010 Oct 19.

Virtual liver resection and volumetric analysis of the future liver remnant using open source image processing software.

van der Vorst JR, van Dam RM, van Stiphout RS, van den Broek MA, Hollander IH, Kessels AG, Dejong CH.

World J Surg. 2010 Oct;34(10):2426-33.

doi: 10.1007/s00268-010-0663-5.

Randomized clinical trial of laxatives and oral nutritional supplements within an enhanced recovery after surgery protocol following liver resection.

Hendry PO, van Dam RM, Bukkems SF, Mckeown DW, Parks RW, Preston T, Dejong CH, Garden OJ, Fearon KC; Enhanced Recovery After Surgery (ERAS) Group.

Br J Surg. 2010 Aug;97(8):1198-206.

doi: 10.1002/bjs.7120.

MRI after chemoradiotherapy of rectal cancer: a useful tool to select patients for local excision.

Engelen SM, Beets-Tan RG, Lahaye MJ, Lammering G, Jansen RL, van Dam RM, Konsten J, Leijtens JW, van de Velde CJ, Beets GL.
Dis Colon Rectum. 2010 Jul;53(7):979-86.

doi: 10.1007/DCR.0b013e3181dc64dc.

A step-up approach or open necrosectomy for necrotizing pancreatitis.

van Santvoort HC, Besselink MG, Bakker OJ, Hofker HS, Boermeester MA, Dejong $\mathrm{CH}$, van Goor $\mathrm{H}$, Schaapherder $A F$, van Eijck $C H$, Bollen $T L$, van Ramshorst $B$, Nieuwenhuijs VB, Timmer R, Laméris JS, Kruyt PM, Manusama ER,

van der Harst E, van der Schelling GP, Karsten T, Hesselink EJ, van Laarhoven CJ, Rosman C, Bosscha K, de Wit RJ, Houdijk AP, van Leeuwen MS, Buskens E, Gooszen HG; Dutch Pancreatitis Study Group.

N Engl J Med. 2010 Apr 22;362(16):1491-502.

doi: 10.1056/NEJMoa0908821.

Human intestinal ischemia-reperfusion-induced inflammation characterized: experiences from a new translational model.

Grootjans J, Lenaerts K, Derikx JP, Matthijsen RA, de Bruïne AP, van Bijnen AA, van Dam RM, Dejong $C H$, Buurman WA.

Am J Pathol. 2010 May;176(5):2283-91.

doi: 10.2353/ajpath.2010.091069.

Epub 2010 Mar 26.

A new model to study intestinal ischemia-reperfusion damage in man.

Derikx JP, Matthijsen RA, de Bruïne AP, van Dam RM, Buurman WA, Dejong $\mathrm{CH}$.

J Surg Res. 2011 Apr;166(2):222-6.

doi: 10.1016/j.jss.2009.09.046.

Epub 2009 Oct 22.

Consensus review of optimal perioperative care in colorectal surgery: Enhanced Recovery After Surgery (ERAS) Group recommendations.

Lassen K, Soop M, Nygren J, Cox PB, Hendry PO, Spies C, von Meyenfeldt MF, Fearon KC, Revhaug A, Norderval S, Ljungqvist O, Lobo DN, Dejong CH; Enhanced Recovery After Surgery (ERAS) Group.

Arch Surg. 2009 Oct;144(10):961-9.

doi: 10.1001/archsurg.2009.170. Review.

Enterocyte shedding and epithelial lining repair following ischemia of the human small intestine attenuate inflammation.

Matthijsen RA, Derikx JP, Kuipers D, van Dam RM, Dejong 
$\mathrm{CH}$, Buurman WA.

PLoS One. 2009 Sep 15;4(9):e7045.

doi: $10.1371 /$ journal.pone.0007045.

Feasibility of randomized controlled trials in liver surgery using surgery-related mortality or morbidity as endpoint. van den Broek MA, van Dam RM, Malagó M, Dejong $\mathrm{CH}$, van Breukelen GJ, Olde Damink SW.

Br J Surg. 2009 Sep;96(9):1005-14.

doi: 10.1002/bjs.6663. Review.

Erratum in: Br J Surg. 2010 Feb;97(2):302.

The effect of a multimodal fast-track programme on outcomes in laparoscopic liver surgery: a multicentre pilot study.

Stoot JH, van Dam RM, Busch OR, van Hillegersberg $\mathrm{R}$ De Boer M, Olde Damink SW, Bemelmans MH, Dejong $\mathrm{CH}_{i}$ Enhanced Recovery After Surgery (ERAS) Group. HPB (Oxford). 2009 Mar; 11 (2):140-4 doi: 10.1111/j.1477-2574.2009.00025.x.

Early endoscopic retrograde cholangiopancreatography in predicted severe acute biliary pancreatitis: a prospective multicenter study.

van Santvoort HC, Besselink MG, de Vries AC, Boermeester MA, Fischer K, Bollen TL, Cirkel GA, Schaapherder $A F$, Nieuwenhuijs VB, van Goor $\mathrm{H}$, Dejong $\mathrm{CH}$, van Eijck $\mathrm{CH}$, Witteman BJ, Weusten BL, van Laarhoven $\mathrm{CJ}$, Wahab PJ, Tan AC, Schwartz MP, van der Harst E, Cuesta MA, Siersema PD, Gooszen HG, van Erpecum KJ; Dutch Acute Pancreatitis Study Group.

Ann Surg. 2009 Jul;250(1):68-75.

doi: $10.1097 /$ SLA.0b013e3181a77bb4.

Mannose-binding lectin null alleles are associated with preserved epithelial cell integrity following intestinal ischemia reperfusion in man.

Matthijsen RA, Derikx JP, Steffensen R, van Dam RM, Dejong $\mathrm{CH}$, Buurman WA.

Mol Immunol. 2009 Jul;46(11-12):2244-8.

doi: 10.1016/j.molimm.2009.04.010.

Epub 2009 May 23.

Rapid reversal of human intestinal ischemia-reperfusion induced damage by shedding of injured enterocytes and reepithelialisation.

Derikx JP, Matthijsen RA, de Bruïne AP, van Bijnen AA, Heineman $\mathrm{E}$, van Dam RM, Dejong $\mathrm{CH}$, Buurman WA.
PLoS One. 2008;3(10):e3428.

doi: $10.1371 /$ journal.pone. 0003428 .

Epub 2008 Oct 17.

Initial experience with a multimodal enhanced recovery programme in patients undergoing liver resection.

van Dam RM, Hendry PO, Coolsen MM, Bemelmans $\mathrm{MH}_{\text {, }}$ Lassen K, Revhaug A, Fearon KC, Garden OJ, Dejong CH; Enhanced Recovery After Surgery (ERAS) Group.

Br J Surg. 2008 Aug;95(8):969-75.

doi: 10.1002/bjs.6227.

Liver volumetry plug and play: do it yourself with ImageJ. Dello SA, van Dam RM, Slangen JJ, van de Poll MC, Bemelmans MH, Greve JW, Beets-Tan RG, Wigmore SJ, Dejong $\mathrm{CH}$.

World J Surg. 2007 Nov;31(11):2215-21.

Perioperative strategy in colonic surgery; LAparoscopy and/or FAst track multimodal management versus standard care (LAFA trial).

Wind J, Hofland J, Preckel B, Hollmann MW, Bossuyt PM, Gouma DJ, van Berge Henegouwen MI, Fuhring JW, Dejong $\mathrm{CH}$, van Dam RM, Cuesta MA, Noordhuis A, de Jong $D$, van Zalingen $E$, Engel $A F$, Goei $T H$, de Stoppelaar IE, van Tets WF, van Wagensveld BA, Swart $A$, van den Elsen MJ, Gerhards MF, de Wit LT, Siepel MA, van Geloven AA, Juttmann JW, Clevers W, Bemelman WA.

BMC Surg. 2006 Nov 29;6:16.

First results after introduction of the four-armed da Vinci Surgical System in fully robotic laparoscopic cholecystectomy.

Heemskerk J, van Dam R, van Gemert WG, Beets GL, Greve JW, Jacobs MJ, Bouvy ND.

Dig Surg. 2005;22(6):426-31.

Epub 2006 Feb 10.

Venous air embolism, preservation/reperfusion injury, and the presence of intravascular air collection in human donor livers: a retrospective clinical study.

Wolf RF, Sluiter WJ, Ballast A, Verwer R, van Dam RM, Slooff MJ.

Transpl Int. 1995;8(3):201-6. 
DANKWOORD 
Caesar. Chef met de kleine kroon. Meester. Vriend. In 1999 stopte ik met mijn opleiding Heelkunde. Medisch innovatief ondernemen in het primaire proces leek toen nauwelijks mogelijk en al helemaal niet als parttime werkende opleidingsassistent. Tijdens de NVGE dagen, laat aan de bar, priem de je je vinger in mijn borst. "JIJ.. MAAKT... EEN... FOUT!". Een fout was het niet. Wat heb ik veel geleerd gedurende mijn reis. En ik ben teruggekomen. Dat had ik zelf ook niet gedacht, maar zo gaat dat bij reizen. Het dankwoord in jouw proefschrift begon met 'in December 1980 stopte ik met de opleiding Geneeskunde'. Inderdaad Kees, we lijken op elkaar! Passie! Rood knopje. Vele interesses. Complexe chirurgie. Radiologie. Tranen. Vooruitgang. Dromen realiseren. Meubels maken. Wijn. De wereld vergeten. Jij wat meer inhoud en wetenschap ik wat meer fantasie en een vleugje commercie. Wat heb ik veel aan jou te danken. Samen opereren over het richeltje van de extreme HPB chirurgie. Pionieren in de minimaal invasieve HPB chirurgie. Na één van de eerste laparoscopische lever resecties riep je: 'We kunnen alles, we hebben het alleen nog niet laten zien'. En zo is het. Ons huidige werk met vijf HPB chirurgen in Maastricht en nog eens vijf in Aken was onvoorstelbaar in 2002. In Maastricht werden in die tijd vijf leveroperaties en enkele alvleesklieroperaties per jaar verricht. Nu meer dan 160, de regio werkt samen. Wat een 'achievement', een jongens droom. Alleen een echte Haruspex kon dat waarschijnlijk voorspellen. Op dit moment zijn we een internationaal meetellend HPB centrum en het eind lijkt nog niet in het zicht. Ik hoop dat het Europese HPB centrum Aken Maastricht binnenkort echt haar deuren opent. In 2004 had je me niet meer kunnen irriteren toen je zij dat de academie niets voor mij was, net zoals je nu af en toe stelt dat ik geen afmaker ben...ik heb nog iets te bewijzen. Gelukkig is daar nog veel tijd voor. Standing on the shoulders of giants!

\section{STEVEN}

Gekke Tukker. Architect. Wat ben je toch een heerlijke kerel en wat ben je soms vermoeiend. Ik heb geloof ik niet eerder iemand ontmoet die zo graag tegen je aanpraat, soms zo weinig ruimte laat en zoveel bevestiging zoekt. Ook Charlie vroeg eens gekscherend 'Did Steve talk to you recently'. 'Hé Ronald, heb je mijn email van 04:30 gezien?' Moet je nou eens zien.....' Met het voorgaande ben je het vast eens en zit je nu je dit leest keihard te lachen. Waarschijnlijk heb je het ook al aan Marja laten zien en zeg je, 'en dat voor een jongen zoals ik'. Kees noemde je ooit de wolf in het koerspeloton, die feilloos weet wanneer hij moet jumpen. En jumpen kan je, Essen, London, Heelkunde lab en M5I. Wat een meesterzet van Kees, dat jij voor mij in ons team belandde. Toen moest ik natuurlijk wel even slikken. Nu weet ik wel beter. Na alle uren, achter onze computers, op onze kamers, de OK, het Heelkunde lab, late taxi's in Edinburgh, Athene en Seoul. Groot Welsden, koe aan het spit, Pipowagen. Met elkaar de wereld veroveren is nog maar net begonnen. De chirurgische Col du Glandon hebben we nu beklommen. Op naar het echte werk. Het wordt weer tijd voor een bootje Sushi en een servetje. 
MARC

BBA8 - Bismuth Bemelmans A8, Ancien de Paris. Wat hebben we een geluk dat we met elkaar werken. Vier totaal verschillende kerels in één lever team. Jij bent onbetwist de snelste leverchirurg in het Maastrichtse. Wellicht op de OK, maar in elk geval in die onwaarschijnlijk snelle auto's van je. Kom, El Dammios, we maken het even snel af. Welles nietes discussies, iedereen wordt er bij gehaald. Na de laatste 7 moeilijke jaren gaan we nu voor 7 vette jaren. In de HPB wereld of gewoon daarbuiten, op de Pietersberg of in het ons zo geliefde Parijs. Nogal eens denk ik, is een Bemelmansiaanse B0-R1 resectie nou een binnenbocht of gewoon verstandig. Met name als we na een dag grensverleggend opereren geconfronteerd worden met acuut leverfalen. De door ons zelf gemaakte B0 classificatie moeten we snel de wereld in brengen.

Gelukkig spreek je goed Nederlands. Ik weet nog goed dat ik je in 2010 in Berlijn op je mobiel belde na het nummer van Christian te hebben gekregen. Met een goede Rudi Carrell klange vroeg ik: 'Hallo, bist Du der neue Chef in Aachen?' Drie dagen later liep je door ons ziekenhuis, het Heelkunde lab en eindigden we de dag in Sjiek. Tot je stomme verbazing een fles Sassicaia op tafel. Je zag er uit alsof je 10 jaar onder een strijkbout had gelegen, wit en vermoeid. Het is ongelofelijk wat je in vier jaar in Aken opgebouwd hebt. Bijna 500 lever en alvleesklier operaties per jaar. Ondertussen begrijpen we dat de Duitse chirurgie cultuur, zeker die van Neuhaus, niet voor Nederlandse vakantie dokters is. Het Europees HPB Centrum lonkt. Aan de slag.

SARIEL

Plant, goeroe, inspirator, ondernemer, 1111 punten plan, Fifteen, Zuidermarkt. Keep going!

MICHAEL

Met hangende pootjes kwam ik terug. Althans, zo werd het links en rechts uitgelegd. 'Get the right people on the bus' zo heb ik het uitgelegd. European Vascular Center, European HPB Center. Het splitsen van de Heelkunde. Laten we doorpakken.

\section{PETER SOETERS}

$\mathrm{Na}$ onze komst in Maastricht, was jij het eerste staflid waarmee Manon en ik, tijdens een afdelings $B B Q$, de tafel deelden. Je stelde geen enkele vraag. Van jou leerde ik luisteren, luisteren, luisteren. Ga zitten naast de patiënt. Een anamnese is in $95 \%$ van de patiënten genoeg om een diagnose te stellen. Om grenzen te verleggen moet je onderzoek doen, de patiënt blijft het middelpunt. Ik had het geluk dat je je laatste jaren in de kliniek wilde slijten. Als je laatste CHIVO heb ik veel van je geleerd. Geduld. Na zes uur opereren in een verkleefde buik zei je bij het eerste darmletsel; 'Ronald, je moet meer geduld hebben'. Je hielp bij mijn eerste ERAS publicatie. Na je vernietigende email met betrekking tot mijn promotie aspiraties is 
het nooit meer helemaal goed gekomen. Ondertussen heb ik door dat er meer gefocust moet worden.

\section{JAN}

2008 Mumbai, Johnny Punch. Who is the star of the show? Toen had ik niet gedacht dat jij in 2012 al zou promoveren. Gelukkig kwam ik je rond het voetbalveld regelmatig tegen en bleef je onnavolgbaar enthousiast uitleggen hoe lekker het is als je klaar bent. 6 coproducties. Nu nog Heelkunde Zuid. Moet kunnen.

\section{ANOUK}

Zelfde verjaardag, hart voor de zaak, net zo eigenwijs, altijd controleren. Check, check, dubbel check. Ik hoop dat je er nog lang bij bent.

\section{MAARTJE}

Ergens staan ze, onze coproducties. 10 - 10 - 10 hebben we niet-niet-niet gehaald. De reis gaat door. Na jou ben ook ik nu aan de beurt. Ik sta de door jou gegeven titel 'most overqualified Doctorandus' weer af. McMumcey, de bank van de wederdienst, Denktank, Boston, HPB. What is the way? Dat zien we wel.

\section{MARIELLE,}

In 2004 liep je binnen. Je wilde aan de slag bij de Heelkunde en aan de slag ging je. Je stond met ons aan de basis van het ERAS lever protocol maar net zo snel als je gekomen was ging je weg naar de Orthopaedie, naar de derde plek op onze publicatie. Gelukkig ben ook jij weer teruggekomen, ben je bijna klaar met je opleiding tot chirurg en promoveer je binnenkort 'fast track' zelf. Chapeau!

\section{JOHANNE, SANNE, MAX, REGINA}

WBMRI en drie keer 'ga terug naar af'. Ik schaar dat onderzoek maar onder de noemer leergeld en lessen in hoe het niet moet. Op naar de next chapter.

\section{TEAM}

Marcel van de Poll, Mechteld de Jong, Rogier van Stiphout, Joost van der Vorst, Simon Dello, Toine Lodewick, Edgar Wong-lun-Hing, Kim van Mierlo, Victor van Woerden, Monique Maas, Robert-Jan Coelen, Ilona Hollander, Stephanie Bukkems, Peter Bollars en studenten. Idserd Klop dank voor al je hulp bij de laatste loodjes. - Together Everybody Achieves More 


\section{MANON, LIEF.}

Het 'ik moet nog aan mijn proefschrift werken' is voorbij. Dank je voor je geduld en zorg voor onze mannen als ik weer de kelder in ging. Wat ben jij een lieve powervrouw. Eerst Groningen, Afrika, Amsterdam, Trambouille en Maastricht. Nu 10 jaar Limme, Dries, Andes, Juul, Tos en Mats. Leven op ons domein op de helling van de Pietersberg. Zoete inval, bijna dagelijks. Weinig is te gek. Al hoewel, soms gaan mijn ideeën je iets te ver. In de afgelopen jaren heb je je vak steeds een beetje aangepast. Kunsteducatie, kunsthandel, kunstadvies, schrijven over kunst. Wat kun jij hard zijn voor jezelf. Niets koffie leuten, 3 boeken in 3 jaar, binnen schooluren, respect! Laat de wereld niet langer wachten. Op weg naar jouw boekenbal.

"Cheshire, would you tell me which way l ought to go from here?"

"That depends a good deal on where you want to get to, Alice"

"I don't much care where so long as I get somewhere,"

"Oh, you're sure to do that if you only walk long enough."

"In that direction or in that direction"

Alice's Adventures in Wonderland

Lewis Caroll 1865 


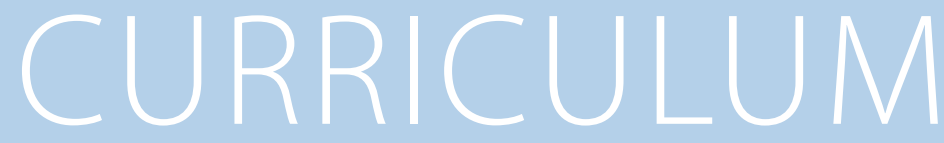

VITAE

RONALD M. VAN DAM 
Ronald M. van Dam was born on July 13th, 1966 in Zeist, The Netherlands as the youngest of three children. In his adolescence, besides playing sports, he learned from his father to use his hands and to get diplomas and from his mother 'be responsible' and 'if you want something, get a job or make it yourself'. Together with his brother his first commercial activities were started in house painting and motorbike refurbishing. His sister showed him creativity and helped to develop his skiing skills.

After graduating from the Atheneum at the Christelijk Lyceum, Zeist in 1985 he travelled for one year with Up With People in the United States of America and the Peoples Republic of China. 'Building bridges of understanding'. In those years he learned the American lessons 'if you want it get it', 'if you fall, stand up again, 'sleeping is for later'.

From 1986 to 1993 he went to Medical School at the Rijksuniversiteit Groningen and was a member of the Groninger Studenten Corps - Vindicat atque Polit. There he observed, 'what is impossible, is still possible'.

He lived at Huize de Ruiker, not more than 900 sidewalk tiles from Mutua Fides and only 750 meters from the Academisch Ziekenhuis Maastricht. Besides singing and discussing life at the bar with his jaarclub Gommes, housemates and friends he was also chairmen of the Hartejongens at the department of cardiothoracic surgery under Prof dr. Jan N. Homan van der Heide and member of the liver transplant group ' Leverboys' under Prof. Dr. Maarten J.H. Slooff.

At he same time he fully renovated and restored a $1500 \mathrm{M}^{3}$ Rijksmonument and turned into a student housing business. While learning negotiating, plumbing, constructing, woodworking and facing a potential bankruptcy he discovered "Giving up is not an option".

In 1993 till his graduation in 1995 he continued Medical School at the Vrije Universiteit Amsterdam. He was winner of the Chirurgendagen Price 1995 for his research project "Anal fissure, digital dilatation or internal sphincterotomy" under Dr. Pieter de Ruiter and Prof. Dr. Bart B. Bijnen at Medisch Centrum Alkmaar.

Early 1996 he started his surgical training under Prof. dr. Gauke Kootstra at the University Hospital Maastricht. In 1999 het continued at the Deaconess Hospital Eindhoven under Dr. Wouter J. Prakken. In 2000, when part time training was not allowed, he stopped and started the ICT company iMedical bv. In februari 2002 he returned to surgical training under Prof. Dr. Michael J.H.M. Jacobs and became CHIVO in Gastrointestinal Surgery under Prof. Dr. Peter B. Soeters in 2004.

Currently he is treasurer of the Dutch Society of Gastrointestinal Surgery - NVGIC, is working 
as HPB surgeon at the Maastricht University Medical Centre and is also a member of the donororgan retrieval team East Netherlands. The European HPB Centre is under construction in collaboration with the Universitätsklinikum Aachen, Germany under Prof. Dr. C.H.C. Dejong and Prof. Dr. U.P. Neumann. 'Focus, perseverance, know what you want, shout it out loud'.

He is father of Limme, Dries, Andes*, Juul*, Tos en Mats and living together with Manon Berns, master of Arts and Arts policy management and currently writer of the Blockbuster book series: The Picasso Mystery, The Secret of Monet and The Sound of Kandinsky, to be published in the near future. 'Listen to what makes your heart beat faster'. 
"The Bird is the best."

"The Bird has zero mortality for 40 years."

Henri Bismuth

Bismuth lecture Capetown, South Africa,

EHPBA 2012

"But remember......it was my idea." 


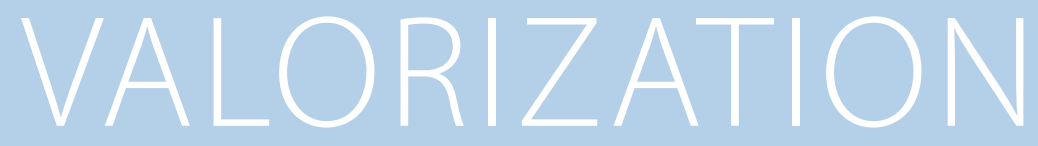

This academic year, the valorization addendum is a new requirement to become $\mathrm{PhD}$. I have been in doubt, with all the clinical work and deadlines I had to meet, to write it. The publisher was counting down; 'you have to defend in 6 weeks'! 
But innovation, valorization and entrepreneurship are among my main interests. From medical school on, I have been telling my friends that the strangest thing on earth is that Dutch medical knowledge institutes and their clinical or scientific staff hardly use inventions, knowledge and intellectual property to create business, patents and income. 'Research is not about money' is a quote echoed by many. Of course it is all about money! You need money for infrastructure, bench fees, inspiring professors and PhD students with a deep-rooted desire to get to the bottom of the problem and discover by strategy and wondering. You also need entrepreneurs dreaming to turn discoveries and solutions into a start-up business.

Valorization. In the academia interest for science and entrepreneurship is not a frequent combination to be found in one person. Stanford University, which I visited in 1985, is still the main example how to turn science into business. Create infrastructure, attract venture capital firms, reward the inventor, assure competition and provide a creative and entrepreneurial environment. Silicon Valley and the large number of partly Stanford owned companies in NASDAQ show the enormous valorization power of Stanford University.

Knowledge transfer. Observing the Dutch landscape of Medical Science, I have been seeing many students doing a PhD program for the sole reason to get into specialty training. After defending their thesis, they never looked into science again. Structurally collecting outcome data a part of process development is too much of a demand for many of those silent PhDs and for management in the hospital industry. Michael R. Bloomberg, former Mayor of New York and $85 \%$ owner of the $\$ 30$ billion company Bloomberg, quoted 'If you don't measure it, you can't manage it'. Today, there is a growing culture of data collection in hospitals mainly forced to comply with government initiated performance indicators. Although the collected data concern mainly quantitative data of how many of something, a start has been made with data on quality or patient reported outcome data.

Entrepreneurship. Back to value of innovation. An example. At the start of this thesis, the Resultaat Verantwoordelijke Eenheid of our department, coincidentally translated by Google Translate as 'The Profit Center', was not interested in financing, 'we don't do science', the implementation of the newly developed ERAS liver protocol. Despite the confidence of the surgical team that it would provide a huge return on investment.

At that time and also today, clinical process optimization or 'development' was mistaken for research. A simple calculation on a napkin made by the apprentice showed that if the hypothesis that implementing an ERAS recovery program in liver surgery would reduce hospital stay from $8-5$ days, the hospital would earn $€ 1.000 .000$,- in 5 years (60 liver resections each year, 3 days, 5 years, $€ 1.100$,- a day). Today, we perform over 100 liver resections in Maastricht and over 250 liver resections in Aachen, annually. 
Worldwide, 1.4 million new patients are diagnosed with colorectal cancer annually. Of these patients, $40 \%$ develop liver metastases and of those $15 \%$ are eligible for liver resection. That means 84.000 liver resections each year. Based on that figure, a modest estimation of the worldwide ANNUAL value of the ERAS liver concept is $€ 277.000 .000$, -. Moreover the number of liver resections for benign lesions or liver cancers is not used in the calculation.

As usual in the academic medical world, the ERAS liver results were published and with help of the inventors, the Dutch Quality Institute CBO implemented ERAS, in the majority of Dutch hospitals. $€ 30.000$, - was reimbursed to cover costs for absence of work/travelling. No patents, no royalties.

10 years later, $65 \%$ of the HPB world is still working with traditional perioperative care programs. That means that a valuable market is still available for education, training, implementation and improvement.

How to continue? 'Research is different from Development'!

Another example. From the databases used for this thesis it has become clear that after liver resection, resection surface related complications occur in $24 \%$ of patients and have a major impact on hospital length of stay. In patients with resection surface related complications the mean hospital length of stay is raised from 5 to over 28 days, resulting in an increase of costs of $€ 23.000$,- per patient. Avoiding these complications can reduce costs substantially and may create a competitive advantage in hospital finance among the 23 Dutch liver centres. However, in the MUMC ${ }^{+}$RVE surgical sciences, there is not a culture of investing in process development or process optimization.

To capitalize on our innovative clinical strategies in surgery we have to start with a taskforce of clinical development. Surgeons and nurses need to be funded to optimize processes. A small percentage of the reimbursement of every surgical patient admitted, needs to be reserved for clinical optimization programs and a clinical innovation playground. Change the devastating culture of ineffective meetings, talking-groups, steering committees, user groups etc. Start to execute. Design products, implement the 1111 philosophy, write short uncomplicated plans, give responsibility and start-up budget for those keen to change and use the companies of the hospital supply chain industry. Become partner, shareholder, developer and innovator in the centre of our industry.

Collaborative networks have already been created worldwide. The HPB staff is ready. The European HPB Centre Aachen Maastricht with over 500 liver and pancreas resections, more than 70 liver transplants and massive potential for growth is only awaiting hospital board investments. 
The value list of this thesis: Extending Indications benefits in more patients eligible, less palliative chemotherapy, less use of biologicals, better quality of life, improved survival, cure for some, higher hospital turnover, larger specialized teams, extended catchment area, less total hospital time, less total costs Liver First benefits in better insight in biologic behaviour of metastases, less unnecessary liver resections, less surgery of the rectum, better quality of life Open Source Liver Volumetry benefits in being easily available and accessible, better estimation of risk for liver failure, better patient selection, improved patient safety, virtual resections at the desktop for planning en training, results from the studies showing shortcomings in the software accelerated the development of a new iNtuition liver template by the manufacturer, OsiriX better suitable, affordable and accessible for research, template development within OsiriX open source community in collaboration with professional OsiriX developers and surrounding technical universities, OsiriX provides state of the art low cost PACS viewer for hospitals usable in a virtual client for all desktops, tablets and smartphones as Application next to SAP ERAS benefits in quicker recovery and shorter hospital length of stay, streamlined perioperative processes, patient participation in recovery, international standardization, uses patients to collect own data in diary, improved patient satisfaction, earlier independence of nursing staff Laparoscopic Liver Resection benefits in further reducing length of stay if combined with ERAS program, shorter operating time in minor liver resections ORANGE IRCT benefits in confirming ERAS liver pilot results, reduces hospital length of stay, shows lack of added value of post operative nutritional support ORANGE II RCTs benefit in marketing value of lead centre in clinical trials, provide insight in real cost-benefit on hospital and society level, standardise perioperative care, improve comparability Composite Endpoint benefits in reducing sample sizes and costs of trials, international standardization, improves comparability. 

Man muss den Dingen

Die eigene, stille,

ungestörte Entwicklung lassen,

die tief von innen kommt,

und durch nichts gedrängt

oder beschleunigt werden kann;

alles ist austragen -

und dann Gebären...

Reifen wie der Baum, der seine Säfte nicht drängt

und getrost in den Stürmen

des Frühlings steht,

ohne Angst,

dass dahinter kein Sommer

kommen könnte.

Erkommt doch!

Aber er kommt nur zu den Geduldigen,

die da sind,

als ob die Ewigkeit vor ihnen läge,

so sorglos still und weit ...

Man muss Geduld haben,

gegen das Ungelöste im Herzen,

und versuchen,

die Fragen selber lieb zu haben,

wie verschlossene Stuben,

und wie Bücher,

die in einer sehr fremden Sprache

geschrieben sind.

Es handelt sich darum, alles zu leben.

Wenn man die Fragen lebt,

lebt man vielleicht allmählich,

ohne es zu merken,

eines fremden Tages

Was mich bewegt

in die Antwort hinein

Rainer Maria Rilke, 1900 
www.ClinicalOptimizationinLiverSurgery.nl 